\title{
Parasite Ecology and the Conservation Biology of Black Rhinoceros (Diceros bicornis)
}

\author{
by
}

Andrew Paul Stringer

\author{
A thesis \\ submitted to Victoria University of Wellington \\ in fulfilment of the requirement for the degree of \\ Doctor of Philosophy
}

Victoria University of Wellington

2016 
This thesis was conducted under the supervision of:

Dr Wayne L. Linklater

Victoria University of Wellington

Wellington, New Zealand

The animals used in this study were treated ethically and the protocols used were given approval from the Victoria University of Wellington Animal Ethics Committee (ref: 2010R6). 


\section{Abstract}

This thesis combines investigations of parasite ecology and rhinoceros conservation biology to advance our understanding and management of the host-parasite relationship for the critically endangered black rhinoceros (Diceros bicornis). My central aim was to determine the key influences on parasite abundance within black rhinoceros, investigate the effects of parasitism on black rhinoceros and how they can be measured, and to provide a balanced summary of the advantages and disadvantages of interventions to control parasites within threatened host species.

Two intestinal helminth parasites were the primary focus of this study; the strongyle nematodes and an Anoplocephala sp. tapeworm. The non-invasive assessment of parasite abundance within black rhinoceros is challenging due to the rhinoceros's elusive nature and rarity. Hence, protocols for faecal egg counts (FECs) where defecation could not be observed were tested. This included testing for the impacts of time since defecation on FECs, and whether sampling location within a bolus influenced FECs. Also, the optimum sample size needed to reliably capture the variation in parasite abundance on a population level was estimated. To identify the key influences on parasite abundance, the black rhinoceros metapopulation in South Africa presented an extraordinary and fortuitous research opportunity. Translocation and reintroduction have created multiple populations from the same two source populations, providing a variety of comparable populations with the same host-parasite relationship. I applied my population-level faecal sampling and egg count protocol to collect 160 samples from 18 black rhinoceros populations over two summer sampling periods between 2010 and 2012. I test hypotheses for the influence of a variety of ecological and abiotic factors on parasite abundance. To test for the influence of individual-level host characteristics on parasite abundance, such as age and sex, I collected rectal faecal samples at the translocation of 39 black rhinoceros. At that time I also investigated the influence of body condition on a variety of measures of host resources, such as the size of sexually selected characteristics. Finally I developed a logical and robust approach to debate whether parasites of threatened host species should be controlled.

For faecal egg counts, samples taken from the centre of faecal boluses did not change significantly up to six hours after defecation. The only factor which significantly affected the size of confidence intervals of the mean parasite abundance for a host population for both parasite groups was the level of parasite aggregation. The accuracy of estimates of mean parasite abundance increased with increasing sample size, with $>9$ samples having little further 
effect on accuracy. As host defecation no longer needs to be observed the efficiency of fieldwork for studies investigating elusive host species is greatly increased. On a population level, host density was the leading model explaining the abundance of both a directly and an indirectly transmitted parasite. For instance, doubling host density led to a $47 \%$ rise in strongyle parasite abundance. I found no support for competing hypotheses, such as climate-related variables, that were thought to affect the abundance of free-living stages of macroparasites. This result will be useful to conservationists as it will allow them to predict where parasite abundance will be greatest and may also reveal potential avenues for parasite control. On an individual level, younger individuals may have harboured higher levels of parasitism ( $\mathrm{p}=$ 0.07). This result would be widely supported by the literature, but a larger host age range is needed to verify the result. I identified four sexual dimorphisms, with anterior horn volume and circumference, and body size, all showing a sex difference in both the slope and intercept of regression lines. Although sexually selected traits are implicated as most vulnerable to parasite impacts, I did not find an influence of parasite abundance on the size of these potentially sexually-selected characteristics or other measures of body condition. This may be because of numerous different factors affecting host resources, of which the parasite groups studied are a relatively small proportion.

Parasites can be an important cause of population decline in threatened species. However, the conservation of potentially threatened parasites within host species is rarely considered. Here, I debated the potential benefits and pitfalls of parasite control to help identify the principles behind parasite control within threatened species. I rank 11 identified different types of parasite control by their potentially detrimental effects on host populations and ecosystems. I conclude that as the risk a parasite poses to host extinction increases, so does the justification for using parasite control methods with potentially detrimental effects. Also, the extinction risk of the parasite should determine the need for dedicated parasite conservation programs. These principles may be predominantly intuitive, but there are a number of examples in the literature where they have not been used, such as the treatment of parasites with low levels of virulence in host species of 'least concern'. The principles provide a framework for the adaptive implementation of parasite control strategies in conservation-reliant species, like rhinoceros.

I embarked on the first multi-population and comparative study of host-parasite relationships in the critically endangered black rhinoceros. This was made possible by empirically testing and refining field sampling protocols to overcome concerns about sample 
identification, number, and age. Through these well-developed, efficient sampling methodologies I was able to determine that host density was the main influence on parasite abundance within black rhinoceros on a population level - a result not previously proven for macroparasites. Influences on individual level variation need further investigation. In particular genetic factors, such as inbreeding, were not researched as part of this study. I successfully identified a number of sexual dimorphisms, but found no evidence that they were influenced by individual parasite abundance. Finally I use a targeted review of the literature to propose some principles behind whether parasites should be controlled within threatened host species. These principles should allow conservation managers to focus resources on those situations where parasite control is needed, and also help conservation managers avoid the potentially detrimental effects of parasite control. In these ways this thesis has advanced the study and understanding of parasites, their ecology, and their relationship to conservation-reliant hosts. 


\section{Acknowledgements}

Many thanks to my parents, family, and friends. In particular Tom Hawkins, Paul Fisher, and Roan Plotz. Wayne Linklater for all his help, advice and eating all the pies. All colleagues at Victoria University of Wellington: Rosalyn Anderson-Lederer, Gaius Wilson, Megan English, Doug Eckery, Heiko Wittmer, Nicola Nelson, Graham Le Gros, Simon Davy, Dalice Sim, Anne Wietheger, Shaun Wilkinson, Luke Cooney, Emma Gibbin, Katie Hillyer, Grace Paske, Stefanie Pontasch, Karl Yager, Jennifer Howe, and the rest of the coffee club. Eastern Cape Parks and Tourism Agency and associated staff: Dean Peinke, Brad Fike, and Gav Shaw. Ezemvelo KZN Wildlife and associated staff: Jed Bird, Geoff Clinning, Tarik Bodasing, Dave Druce, Bhom Ndondwe, Joseph, Chris Barichievy, Pete Ruinard, Petros Ngwenya, Catherine Hanekom, Dennis Kelly, Dave Robertson and Suni Raas. Wildlife Act: Chris Kelly, Simon Morgan, and Cathy. A special mention to the ineffable Brendan Whittington-Jones. All friends and colleagues who filled in the online body condition survey. Many thanks for the valuable assistance from Nelson Mandela Metropolitan University including Graham Kerley, Shirley Parker-Nancy, Craig Tambling, Liaan Minnie, Diane Smith, and Marietjie Landman, South African National Parks capture unit including Dave Zimmerman, Cathy Dreyer, Markus Hofmeyr, Tosh Ross, and Hanno Kilian, the South African Weather Service, the Rhino Resource Center, Annemieke van der Goot, WWF and Jacques Flamand. Very special thanks to the owners, staff and black rhinoceros monitors from numerous private reserves who wished to remain anonymous due to the recent upsurge in rhinoceros poaching.

Many thanks to Anne La Flamme, Nancy Webber and Stephen Hartley for their very useful comments on final drafts of the manuscript. I would also like to say hello to Jason Isaacs. 


\section{Contents}

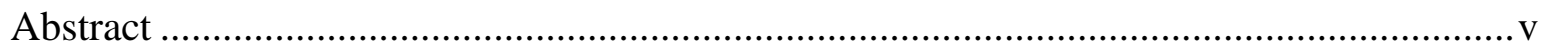

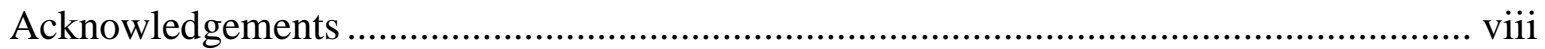

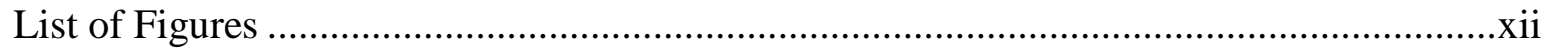

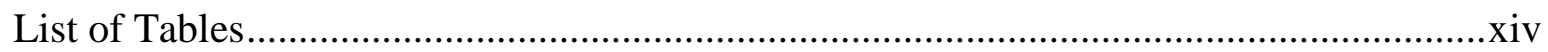

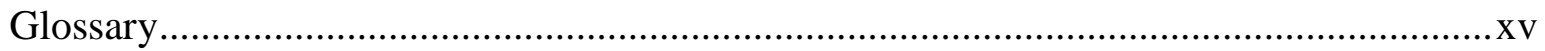

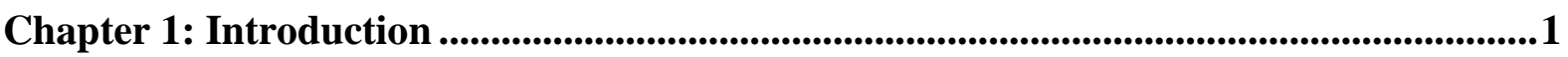

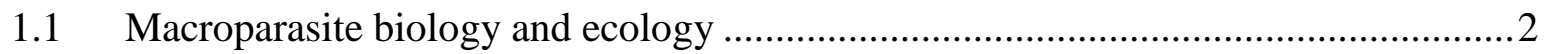

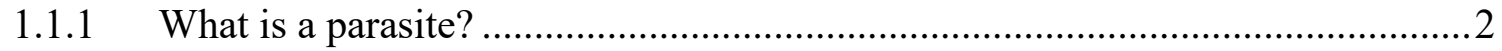

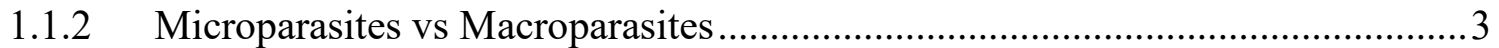

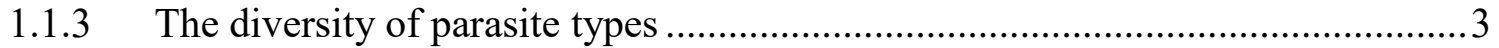

1.2 Epidemiology \& evolution of virulence in macroparasites .......................................6

1.2.1 Distribution and epidemiology of macroparasites ........................................... 6

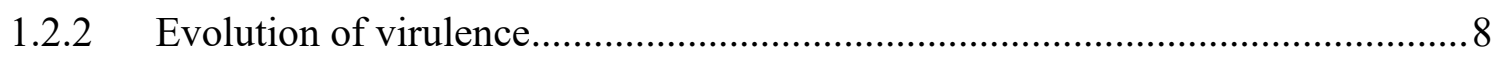

1.2.3 Native vs non-native parasites, and host-switching ......................................... 11

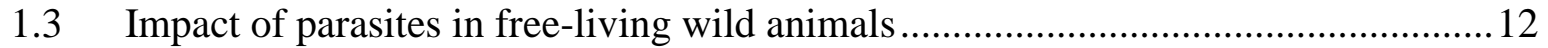

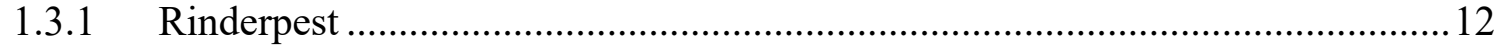

1.3.2 Chronic infections and the interactive effects of polyparasitism.......................13

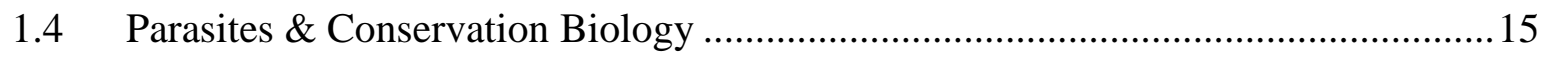

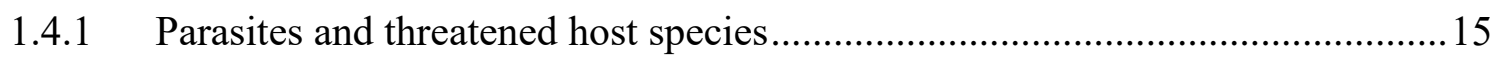

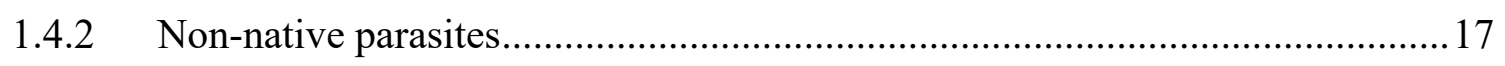

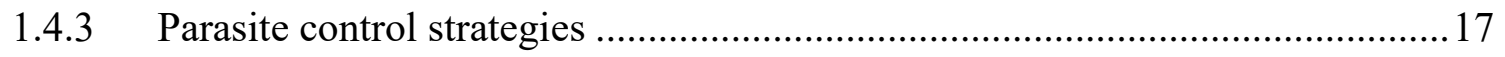

1.5 The biology, ecology, and conservation of black rhinoceros................................. 19

1.5.1 The biology and ecology of black rhinoceros ................................................. 19

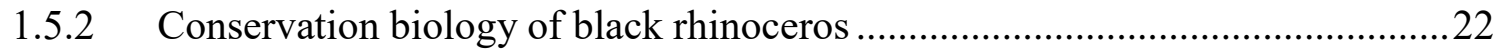

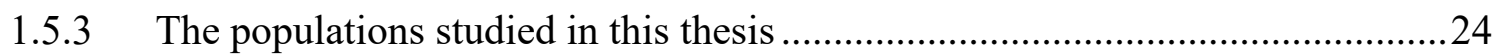

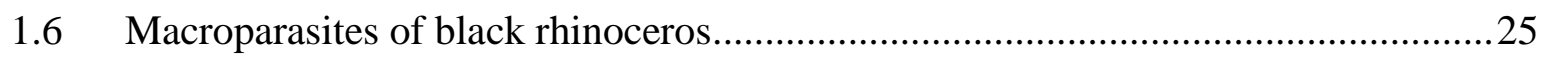

1.6.1 Previous work on the parasites of black rhinoceros.......................................25

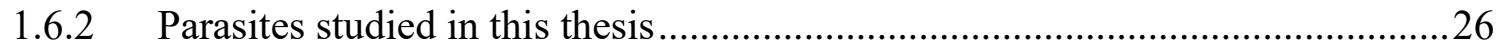

1.6.3 The quantification of parasites in black rhinoceros ........................................28

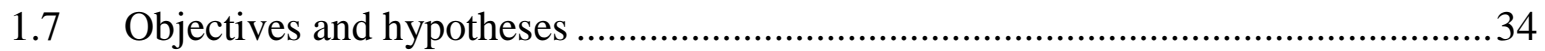

Chapter 2: Reducing sampling error in faecal egg counts from black rhinoceros

(Diceros bicornis) ...........................................................................................................................39 


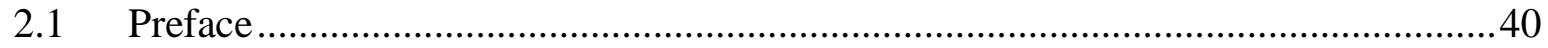

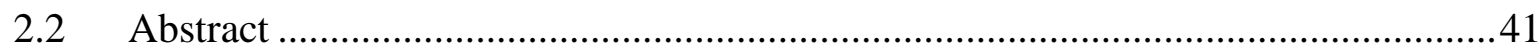

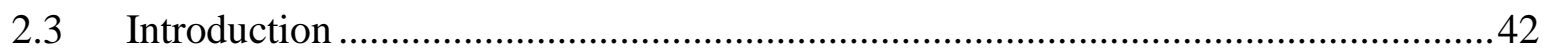

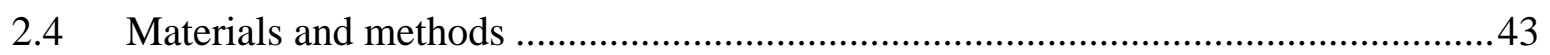

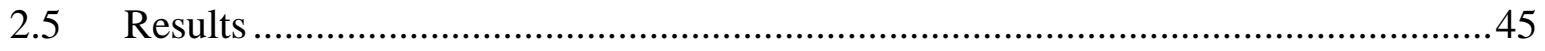

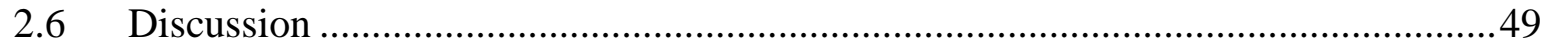

Chapter 3: Host density drives macroparasite abundance across populations of a

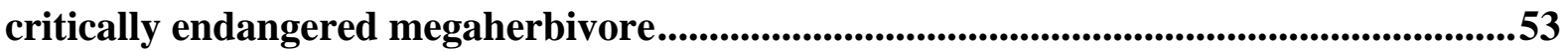

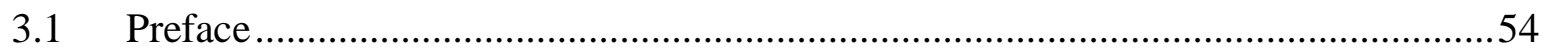

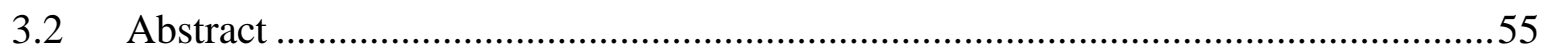

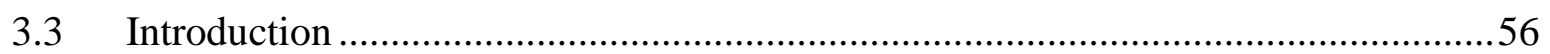

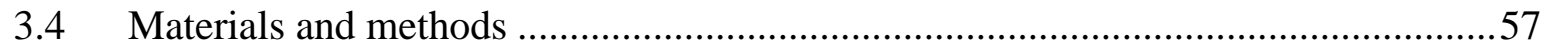

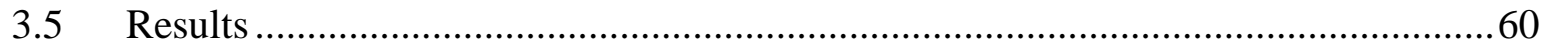

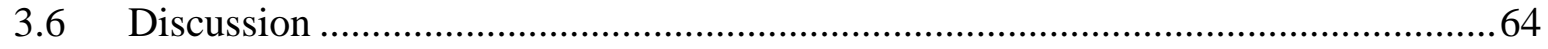

3.7 Post-publication addendum about sampling and analysis robustness ......................67

Chapter 4: Investigating the effects of parasitism on the body condition and sexual development of black rhinoceros (Diceros bicornis) ..........................................................73

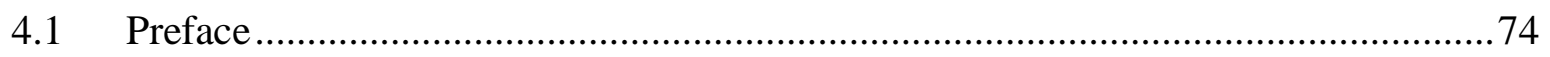

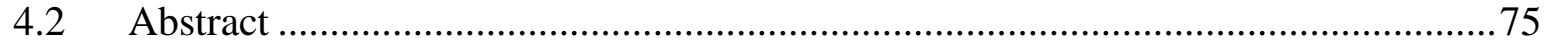

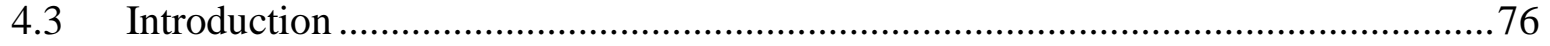

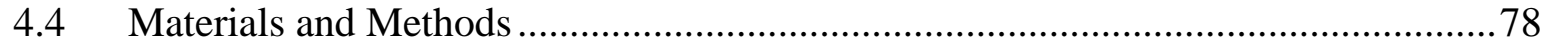

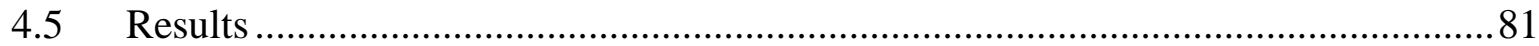

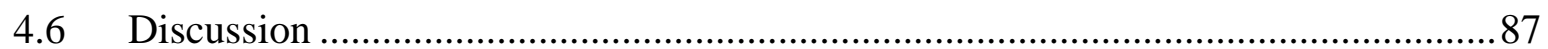

Chapter 5: Everything in moderation: Principles of parasite control for wildlife

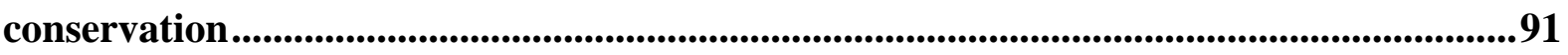

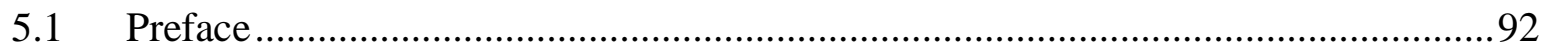

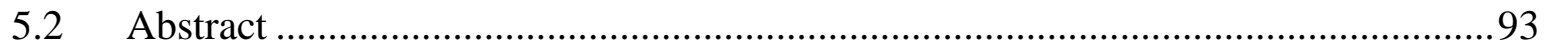

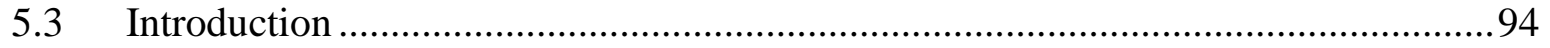

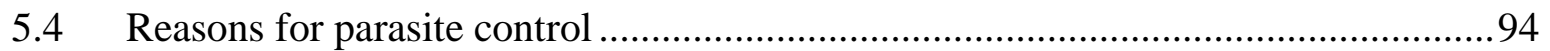

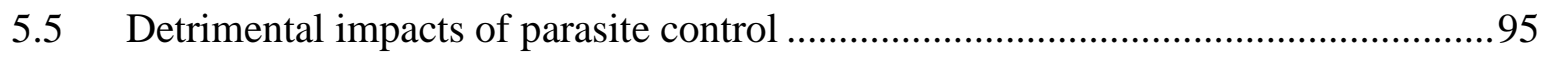

5.6 A proposal for the principles of parasite control in threatened species ...................96

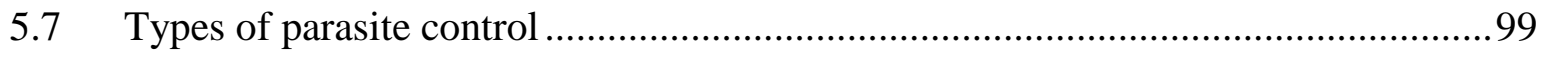

$5.8 \quad$ Past examples of parasite control .................................................................. 100 


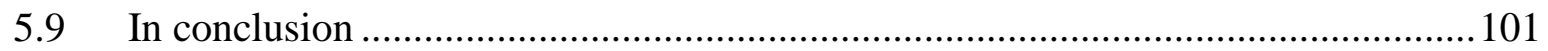

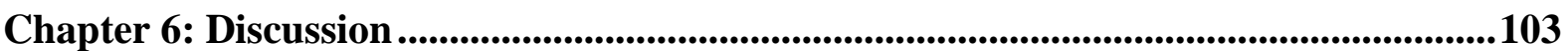

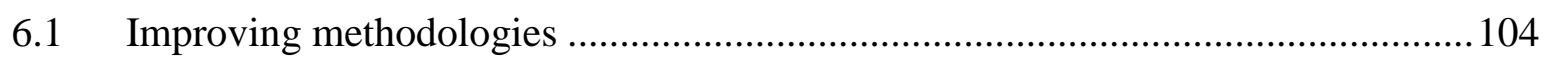

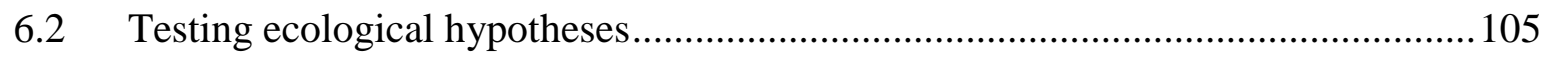

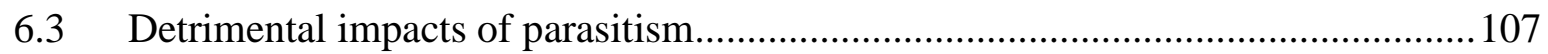

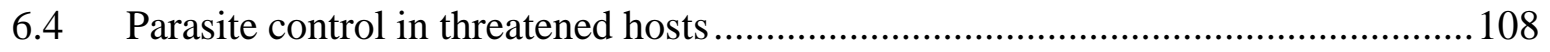

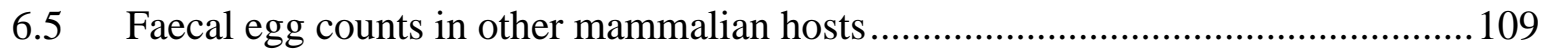

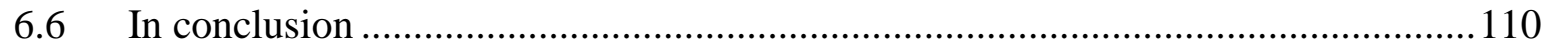

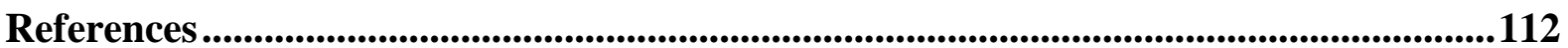

Appendix 1: The effect of host capture on faecal egg counts ............................................ 135

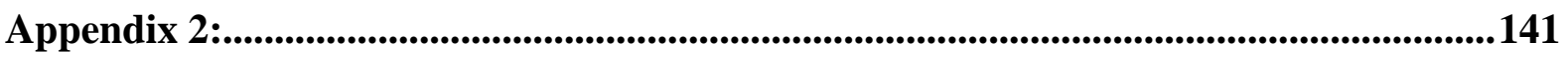

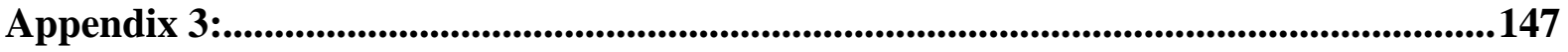

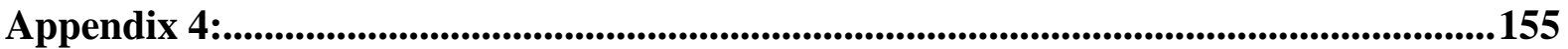




\section{List of Figures}

Figure 2.1 Mean faecal egg counts sampled 3, 6, \& 9 hours after initial collection from captive black rhinoceros.

Figure 2.2 The distance from the mean of bootstrap 90\% confidence intervals against sample size for faecal egg counts from 18 black rhinoceros populations.

Figure 2.3 The accuracy of the estimated mean after each sampling event for each population with 10 or more samples. The corrected moment estimate of $k$ and the population mean are given in the legend.

Figure 3.1 Scatter plot showing mean strongyle abundance in host populations against the density of black rhinoceros in that population.

Figure 3.2 Scatter plot showing mean Anoplocephala sp. abundance in host populations against the density of black rhinoceros in that population.

Figure 3.3 A histogram of the distance to the closest sample from each sample.

Figure 3.4 How samples in close proximity to each other affect the fitted model between host density and parasite abundance.

Figure 3.5 How removing populations with low sample size affects the fitted model between host density and parasite abundance.

Figure 4.1 The estimated strongyle parasite intensity from rectal faecal samples of captured black rhinoceros of different ages.

Figure 4.2 Sexual dimorphisms identified in black rhinoceros. The size of the sexual dimorphism is plotted against the age of individuals.

Figure 4.3 The correlation between two measures of body condition derived from body measurements - the residuals of the regression of body weight to body length, and half girth to body length.

Figure 4.4 The correlation between two measures of body condition - the residuals of the regression of half girth to body length, and a visual assessment of body condition.

Figure 4.5 The correlation between parasite intensity or prevalence and body condition as measured by the residuals from the regression of half girth to body length. 86 
Figure 4.6 The correlation between parasite intensity or prevalence and a visual assessment of body condition.

Figure A1 Faecal egg counts taken from samples collected from black rhinoceros brought into captivity. 


\section{List of Tables}

Table 1.1 Studies investigating the correlation between FECs and intestinal parasite abundance in sheep (Ovis aries), goats (Capra aegagrus hircus), cows (Bos taurus), moose (Alces alces), and horses (Equus caballus).

Table 3.1 Descriptive statistics from the environmental parameters used in candidate models.

Table 3.2 Information-theoretic table of candidate models explaining strongyle parasite abundance as a function of host rhinoceros density.

Table 3.3 Information-theoretic table of candidate models explaining Anoplocephala sp. abundance as a function of host rhinoceros density.

Table 4.1 Sexual dimorphisms in black rhinoceros.

Table 5.1 Different parasite control strategies are classified into one of three broad categories - ecological restoration, ecologically invasive strategies, and strategies that alter the host-parasite arms race. 


\section{Glossary}

Disease

"A condition of the body, or of some part or organ of the body, in which its functions are disturbed or deranged;" (OED, 2015a)

Parasite "An organism that lives on, in, or with an organism of another species, obtaining food, shelter, or other benefit; (now) spec. one that obtains nutrients at the expense of the host organism, which it may directly or indirectly harm." (OED, 2015b).

Parasite intensity The number of parasites within infected hosts only (Bush et al., 1997).

Parasite prevalence The proportion of a host population that is infected with a parasite (Bush et al., 1997).

Parasite abundance The number of parasites within a population, including both infected and uninfected hosts (Bush et al., 1997).

Virulence "The property or quality of being physically virulent or full of virus; extreme poisonousness or venomousness; malignity or violence (of disease)." (OED, 2015c). I use virulence in this thesis as the level of detrimental impacts on individual hosts.

Elusive species Difficult to find species that are rarely seen in the wild and hence difficult to directly observe. 


\section{Chapter 1: Introduction}

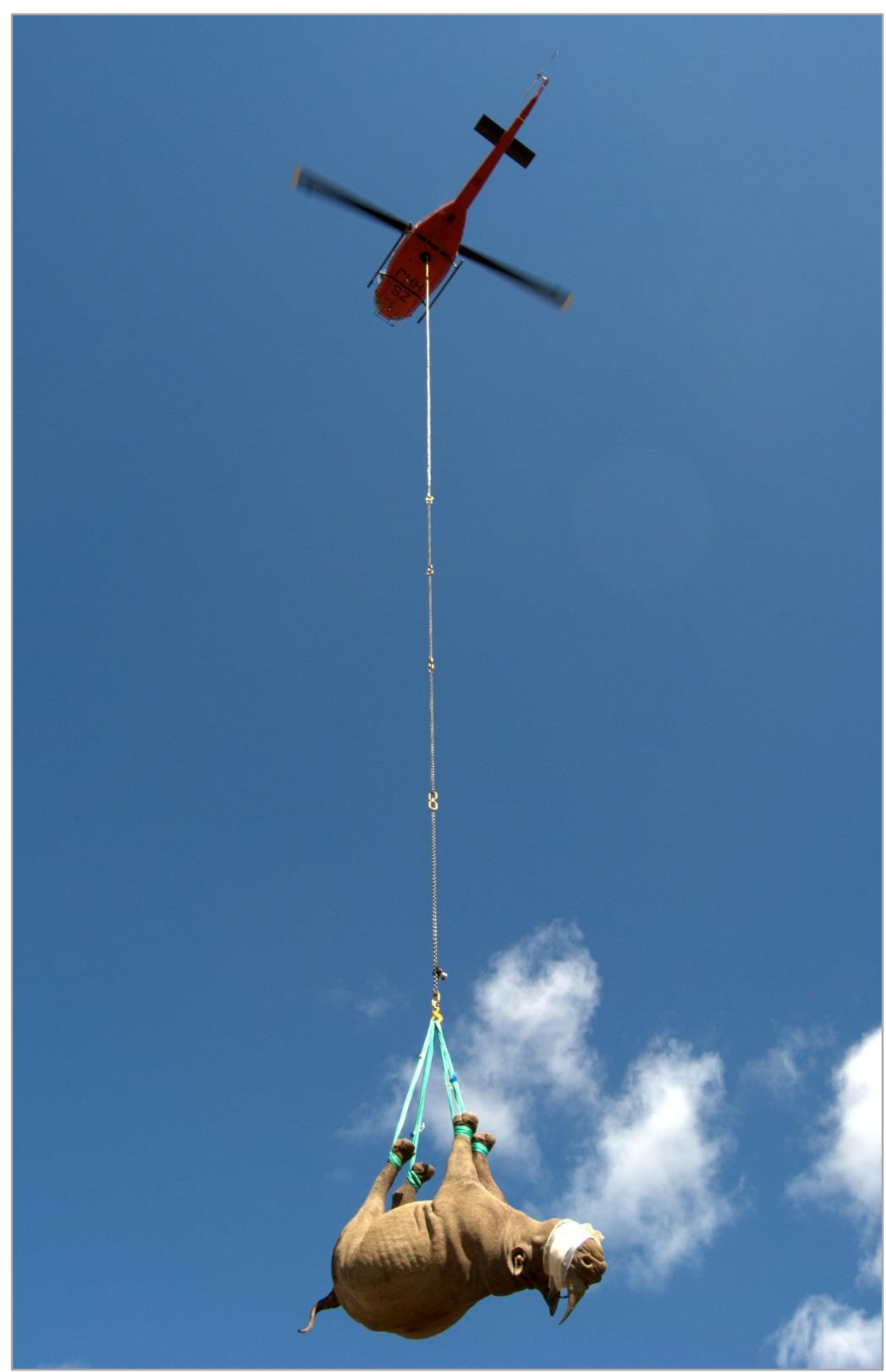

Black Rhinoceros range expansion project (WWF), Eastern Cape, Nov 2012, (C) Andrew Stringer.

"The ugliness and repulsiveness of the rhinoceros are of course a matter of taste. Anyone who, like myself, has had repeated opportunities of studying the animal closely will soon discover the attractions of this giant.” Joseph Delmont (1931) 


\subsection{Macroparasite biology and ecology}

\subsubsection{What is a parasite?}

In this $\mathrm{PhD}$ I use the term parasite in its widest interpretation, which is a species which lives on or in a host from which it derives resource at the expense of the host (see glossary). Parasites vary in the type, severity, and frequency of their negative effects. In this $\mathrm{PhD}$ I use the term virulence to describe those negative effects (see glossary) (Read, 1994). Virulence, however, is not a fixed parasite trait as different strains of the same parasite species may have differing levels of virulence (see 1.3.1). Virulence is also the consequence of a host-parasite interaction. Thus, a parasite that is not, or subtly, virulent in one host, for a variety of reasons such as host resistance, may be highly virulent in another host (e.g. ebolavirus, Pourrut et al., 2007). The impacts of virulence are also not analogous with parasitic impacts on host population performance. For instance, a parasite with low levels of virulence, that causes chronic infections, and is found in all individuals within a population, has the potential to have as similar an effect on population performance as a highly virulent parasite which only has acute effects in a small proportion of a population (see 1.4). Hence, numerous factors may influence the effects of a parasite on their host population.

Some parasites may be host specific, highly preferential towards certain hosts, or infect a range of hosts. The tick species Amblyomma rhinocerotis, for example, is found predominantly, but not exclusively, on black and white rhinoceros (Rhinocerotidae) (Penzhorn et al., 1994). Some parasites only infect a single host species. For instance, bat flies (Diptera: Streblidae and Nycteribiidae) are known to be host specific, despite frequent interaction with species similar to their hosts (Dick and Patterson, 2007). Being host specific has a number of benefits for a parasite, such as being able to adapt to a single species host immune system. However, some parasites may also infect a range of host species. The generalist tick Amblyomma hebraeum, for example, feeds on a wide variety of different ungulate hosts, including rhinoceros. This generalist strategy means a parasite may more easily find a host (Norval et al., 1989; Leggett et al., 2013).

Parasite transmission may be direct from host to host, or indirect whereby the parasite utilises one or more intermediate hosts, or utilises a vector to transmit from one host to the next. Indirectly transmitted parasites may be host specific for some or all of their life cycle. For instance, all trematodes have at least two hosts as part of their lifecycle, one of which is always a mollusc (Schmidt and Roberts, 2005). The host in which a parasite reproduces sexually is 
known as its definitive host. For instance, the definitive host of Plasmodium spp is its mosquito host (Anopheles spp) (Schmidt and Roberts, 2005).

\subsubsection{Microparasites vs Macroparasites}

Parasites can be divided into either microparasites or macroparasites. Broad parasite characteristics are used to assign a parasite into either group, but exceptions are common. Macroparasites and microparasites may be differentiated based on size. Microparasites are generally unicellular and macroparasites multicellular (e.g. Dieckmann et al., 2005). Fungi are usually classed as microparasites. However, fungal parasites may be both unicellular, such as Nosema apis, and multicellular, such as Cordyceps spp (Higes et al., 2006; Sung et al., 2007). Macro- and micro-parasites might also be differentiated by their reproductive strategy. Microparasite multiplication often occurs within a host (autoinfection), while macroparasite offspring usually go on to infect a different host individual than their parents (Dieckmann et al., 2005). However, there are some species of macroparasites, such as the nematode Probstmayria vivipara, that will readily autoinfect the same host as their parents (Smith, 1979). Microparasite infection may also be intracellular, a strategy not available to macroparasites due to their size. This leads to an important epidemiological difference, in that some microparasites can transmit vertically and directly into the offspring of an infected host (Dieckmann et al., 2005). Dividing parasites into microparasites and macroparasites, then, is useful due to the gross differences in infection pathway and residence between the groups. However, as the groups are not defined along taxonomic boundaries, the defining features of macroparasites and microparasites are somewhat indistinct. Attempting to define laws that categorise and group parasites is fundamentally difficult due to the diversity of parasites.

\subsubsection{The diversity of parasite types}

Parasites are an abundant component of biodiversity, with an estimated $40 \%$ of all known species thought to be parasitic (Dobson et al., 2008). Indeed, parasite biomass within certain ecosystems has been found to exceed the biomass of all other top predators (Kuris et al., 2008). Parasites are found within a wide variety of taxonomic groups, ranging from viruses to birds. Here I provide some examples of parasitism from these groups, to illustrate the diversity of parasite types that exist. 


\subsubsection{Microparasites}

The smallest microparasites are viruses, such as the canine distemper virus and rhinoviruses - the cause of the common cold. All viruses are obligate parasites and replicate only within host cells. Viruses affect a huge variety of different hosts, from megafauna to bacteria.

Bacteria are also classed as microparasites. These include the species that cause anthrax (Bacillus anthracis), cholera (Vibrio cholerae), and lyme disease (Borrelia burgdorferi). Bacteria have a range of different strategies for transmission between species. For instance, $B$. anthracis has a spore that can survive in the environment for many decades, $V$. cholerae transmission is direct (faecal-oral), while B. burgdorferi relies on a vector, ticks, to transmit from host to host. These species also have highly variable levels of virulence. For instance, $B$. anthracis is severely detrimental to its hosts due to the production of a microbial toxin, conversely, the symptoms of lyme disease may be low level in comparison but longer lasting (Mock and Fouet, 2001; Wormser et al., 2006).

There are a number of unicellular protozoa that may be parasitic. These include parasites of the blood such as the piroplasms Babesia spp \& Theileria spp, Plasmodium spp (which cause malaria), and parasites of the intestine such as Giardia spp. These may have a variety of transmission methods. For instance, Babesia, Theileria, and Plasmodium use arthropod vectors, while Giardia transmission is direct (faecal-oral). The causes of virulence also differ between these species. While Plasmodium are detrimental to their hosts due to the parasites' replication within host red blood cells, destroying the cell, Giardia instead utilises host food resources, and may also cause diarrhoea.

\subsubsection{Macroparasites}

\section{Platyhelminths}

A number of species of platyhelminths are obligate parasites. These include the Monogenea, (usually ectoparasites on the gills of fish), the Trematodes (endoparasitic flukes), and the Cestodes (tapeworms) (Buchmann and Lindenstrøm, 2002; Schmidt and Roberts, 2005). An interesting example of a trematode is Dicrocoelium dendriticum, a parasite found in the liver of many ruminants. It has the ability to change the behaviour of its intermediate hosts, an ant, and often Formica spp. It causes the ant to attach to stems of grass at night to increase 
the likelihood that it is eaten by its definitive host, an ungulate (Manga-González et al., 2001). Indeed, there are a number of examples of parasites with the ability to change host behaviour (Poulin, 2000).

Cestodes are commonly known as tapeworms. Almost all have an indirect life-cycle, although many of their life-cycles have yet to be directly studied. In their definitive host they live in the intestine and attach to the gut wall via a scolex (Schmidt and Roberts, 2005). This attachment may be detrimental to its host due to damage to the intestinal wall, especially when there is a high intensity of infection within a host (Zajac and Conboy, 2006). The tapeworm takes resources away from its host by absorbing nutrients from the digesting food within the gut. Hence, in some ways it could be called a kleptoparasite - a parasite that steals food from another individual.

\section{Nematodes}

The nematode worms are a broad group thought to have become abundant in nearly every habitat on earth. While there are many species of nematode that are free-living within aquatic or soil environments, numerous species of nematode are parasitic. They may be directly, or indirectly transmitted, and may live in a variety of locations in the host. For instance, the strongyles (Strongylida) are usually directly transmitted, and found in the gut of a wide range of vertebrate hosts. In contrast, the filarial nematodes (Filaroidea) live in the tissue of their hosts, are indirectly transmitted, and utilise an invertebrate intermediate host. Interestingly, the majority of filarial nematodes have an obligate symbiotic relationship with bacteria from the genus Wolbachia (Schmidt and Roberts, 2005). Nematodes have developed a range of ways to complete their lifecycle. For instance, the nematode Myrmeconema neotropicum causes its ant intermediate host to resemble a ripe fruit, increasing the likelihood that it will be eaten by its bird definitive host (Yanoviak et al., 2008).

\section{Other invertebrates}

There are many ectoparasitic invertebrates, including ticks and mites (Parasitiformes), lice (Phthiraptera), mosquitos (Culicidae), and leeches (Hirudinea). Of particular note is the tongue-eating louse (Cymothoa exigua), which feeds on the tongue of its fish host, ultimately replacing the tongue completely (Ruiz and Madrid, 1992). 
Some species are parasitic for only a part of their lifecycle, such as the freshwater pearl mussel (Margaritifera margaritifera). M. margaritifera adults are filter feeders of many fast flowing streams in Europe. However, juvenile stages are ectoparasites on the gills of some salmonids. Interestingly, parasitism not only helps $M$. margaritifera juveniles grow, but also assists with upstream dispersal (Hastie and Young, 2001).

\section{Vertebrates}

Brood parasitism is where a species tricks another species into raising its young as their own. This means that a host provides the required parental investment, often at the expense of the host's own young. This has been observed to varying degrees in a number of bird species, of which the most commonly cited are the cuckoos (Cuculidae). However, the strategy is also used by some invertebrates such as the cuckoo bees (Nomadinae). Interestingly, brood parasitism may also occur intra-specifically, between individuals within the same species, as observed in a number of duck species (Anatidae) (Sorenson, 1997).

There are also other forms of vertebrate parasites. For instance, the jawless fish river lamprey (Lampetra fluviatilis) take large chunks of flesh from their prey/host (other fish species), but the prey/host often does not die (Potter and Hilliard, 2009). There are also a number of examples of kleptoparasitism within the vertebrates, such as spotted hyenas (Crocuta crocuta), which will steal food from other predators (Macdonald, 2006).

\subsection{Epidemiology \& evolution of virulence in macroparasites}

\subsubsection{Distribution and epidemiology of macroparasites}

Macroparasites are unevenly distributed through a host population. Primarily they are aggregated within a small proportion of the population, so that a small proportion of hosts harbour the majority of the parasite population. For instance in a meta-analysis, $90 \%$ of studies showed a parasite population with a clear negative binomial distribution (Shaw et al., 1998). There are some exceptions to this rule, in particular the nematode Pterygodermatites peromysci is extraordinary by being normally distributed through their host population, the white-footed mouse (Peromyscus leucopus) (Luong et al., 2011). Parasite aggregation may be caused by a range of factors, however a key cause of aggregated distributions is likely to be variation in host immunity (Galvani, 2003; Boag et al., 2001; Johnson and Hoverman, 2014; Morrill and 
Forbes, 2012). A range of other factors have been proposed that may affect parasite aggregation, and these are intrinsically linked to determinants of individual parasite intensity (the abundance of parasites within an individual, see glossary). Parasite intensity is known to vary between years and seasons, and with host age, sex, behaviour, and the prevalence of other parasites (Boag et al., 2001; Johnson and Hoverman, 2014). Seasonal and annual variation is attributed to weather conditions, which might affect the survival of free-living parasite stages, or hosts and vectors (Boag et al., 2001; Cattadori et al., 2005b). It has been proposed that the survival of these stages directly influences infection rate, and hence ultimately population-level parasite abundance (Grenfell and Dobson, 1995).

The abundance of parasites within an individual is thought to increase with age as an individual's exposure to different parasites should increase with time. However, a host may develop an immunity to an infection, and hence parasite abundance may asymptote, or reduce after a peak (Woolhouse, 1998; Cattadori et al., 2005a). This peak in parasite abundance is known to shift with transmission rate, with parasite abundance being higher and earlier for populations with high levels of transmission (Woolhouse, 1998). This is discussed further in Chapter 4.

Male vertebrates may harbour a greater parasite abundance than female. However, this effect is not ubiquitous across all species of host or parasite, and indeed the effect is usually small (Poulin, 1996; Schalk et al., 1997; McCurdy et al., 1998). The differing effects of sex hormones on the immune system may contribute to the difference in parasite abundance between sexes, but there may also be other causes, such as behavioural differences between sexes that modify exposure to parasites (Schalk et al., 1997).

It is thought that some individuals have an innate predisposition to parasitism in comparison to others (Holland, 2009; Hayward, 2013). This has been shown to be at least partially due to an individual's genetics that underlie the immune response (Paterson et al., 1998; Stear et al., 2007). Also, poor body condition and low host nutrition may interact with immune response to determine the level of individual susceptibility to parasite infection (Hughes and Kelly, 2006; Hayward, 2013; Beldomenico et al., 2008).

Inbreeding may be an important mechanism underlying an individual's genetic predisposition to parasitism, as relatively more inbred individuals have a higher parasite burden (Coltman et al., 1999). Indeed, inbreeding may be an important mechanism determining parasite abundance on the population level as well (Cassinello et al., 2001). This is because a 
genetically highly homogeneous population may allow a parasite to adapt to that population, and hence infect novel individuals more easily (Lively and Dybdahl, 2000).

The transmission rate of a parasite is also thought to be a key determinant of its abundance within a host population. Transmission is often thought of as either densitydependent, or frequency-dependent. Density-dependent transmission assumes that the rate of contact between infected and susceptible individuals is directly related to the density of individuals. Hence, at high population densities the contact rate between individuals is high, resulting in a high rate of transmission. The opposite is true at low population densities. However, the key assumption of density-dependence is that host density and contact rate are directly related. When contact rate is independent of density, a parasites' transmission may better be described as frequency-dependent (reviewed in McCallum et al. 2001). A common example of frequency-dependent transmission is that of sexually-transmitted parasites, where transmission may be dependent on the frequency of copulations rather than the density of hosts (May and Anderson, 1987; Lloyd-Smith et al., 2004). However, there may be numerous other situations where frequency-dependent transmission may occur. To hypothesise, beavers (Castor spp.) control strict family territories. However, these territories are often two dimensional (along a watercourse). An increasing number of family groups may result in smaller territories (Campbell et al., 2005) but not increase contact rates as the majority of contact will still be confined to upstream and downstream territory borders. Hence, an increasing density of beavers may not increase contact rates.

Here I have outlined a variety of factors that may affect parasite abundance. However, the relative importance of abiotic factors, such as climate, and biological factors, such as an individual's predisposition to parasitism and transmission rates, is unknown (Tompkins et al., 2011). For instance, some factors may be highly influential on the population level, while others may only influence variation between individuals within a population. It is also likely that these factors will influence macroparasites differently, depending on whether they have free-living stages or intermediate hosts and the behaviour and ecology of those hosts.

\subsubsection{Evolution of virulence}

Initially it was thought that as a parasite relies on its host to survive, a parasite should evolve towards avirulence due to the benefit the host provides. However, this has now been recognised as only one factor in a complex combination of potential influences (Anderson and 
May, 1982; Méthot, 2012). A key additional mechanism is described by the trade-off hypothesis. This argues that an increase in virulence will decrease host longevity, but also lead to an increase in the reproductive rate of a parasite. Hence, it is proposed that parasite virulence is a trade-off between parasite reproductive rate and host longevity (Alizon et al., 2009).

There are numerous evolutionary pressures that may impact any trade-off between parasite reproductive rate and host longevity (Alizon et al., 2009). For instance, many parasites may be transmitted horizontally (between hosts), or transmitted vertically (from parent to offspring). If a parasite is transmitted vertically then decreasing even the ability of its hosts to reproduce will also be directly detrimental to the parasite's own reproduction, as it reduces the number of hosts for the next generation to directly infect (Yamamura, 1993). This is akin to an expansion of the avirulence hypothesis in that it should drive a parasite towards fewer detrimental effects. An example of this is Wolbachia, which are found within a variety of arthropods, have low levels of virulence, and are predominantly vertically transmitted. Unsurprisingly therefore, studies have shown that Wolbachia can be either parasitic or mutualistic (Lipsitch et al., 1996; Zug and Hammerstein, 2014). Wolbachia must trade-off between increasing its own virulence and hence increasing its own reproductive rate, with the detrimental impacts virulence has on its host's reproductive rate, and hence the success of the next generation. This trade-off is particularly pronounced for Wolbachia as parasite virulence will directly affect the hosts of its own offspring (as it is vertically transmitted), rather than only the wider host population that may be affected by a parasite that is not vertically transmitted.

The trade-off hypothesis requires a parasite species to be able to evolve towards avirulence, but proposing mechanisms for how this may occur has proved to be difficult. Within a species, if a single individual parasite carries a gene for reduced virulence and hence reduced reproductive rate, this individual would be out competed by other individuals from the same species that did not have reduced virulence. This is because any resulting increase in host longevity would benefit all parasites within the host, but the cost would be borne by a single individual parasite. Conversely, if a single individual had a gene for greater virulence, that individual would have a competitive advantage over all others, as it gains the benefit from their less virulent strategies and has a higher reproductive rate (Frank, 1996). This seems to indicate that due to competition, parasites should evolve towards greater virulence. Indeed, there is some evidence for this as more virulent rodent malaria (Plasmodium chabaudi) strains have a competitive advantage in concurrent infections (De Roode et al., 2005). This within-species 
interaction may also occur between parasite species (López-Villavicencio et al., 2011). The parasite community within a host may be a diverse selection of parasite species and individuals. If the trade-off hypothesis holds true, it may be expected that a less virulent species would be outcompeted when in competition with other similar parasites that are of higher virulence. However, this cannot be the only explanation of parasite virulence as there are many parasites with low levels of virulence that are highly successful as a parasite (exist in high abundance), such as Toxoplasma gondii (Schmidt and Roberts, 2005).

There are a large range of further factors that complicate this simplistic view of parasite competition. For instance, some nematodes trigger host immunity towards conspecific larvae that would have competed for the same resource (the host), hence limiting parasite abundance within the host, and increasing individual parasite success (Brown and Grenfell, 2001). This competition is most pronounced when a host infected with a chronic parasite infection is also infected by an acute, highly virulent parasite (Balmer et al., 2009). Indeed, it has been shown that infection by microsporidian parasites (that have low levels of virulence) of Daphnia magna, protects against infection from highly virulent bacterial infections (Lange et al., 2014).

The optimal trade-off between host longevity and reproductive rate will change depending on the number of parasite competitors within a host. Indeed, a parasite may modify its own strategy to take into account the reduction in host longevity caused by other parasites that are present. This developmental plasticity in the level of virulence has also been observed (López-Villavicencio et al., 2011).

Parasites can also reduce their virulence when a host is under stress to prolong host longevity. This has been observed in closely related protozoa, where parasite apoptosis is observed to reduce parasite burden (Reece et al., 2011; Pollitt et al., 2010). Host longevity may also be particularly important for long-lived parasites that cause chronic infections. For instance, the tapeworm Diphyllobothrium latum can survive for 25 years within a host (Loker and Hofkin, 2015). Of interest is whether these parasites can reduce their virulence in an attempt to improve host survival during temporal stress (e.g. to improve host over-winter survival) (see Appendix 1 where I found substantial declines in parasite egg counts in faeces after rhinoceros capture).

Arguments against hypotheses for evolution towards avirulence, then, closely resemble arguments against group selection and altruism (Frank, 1992; Frank, 1996). Hence, any theory that purports an evolution towards avirulence must be backed up by a mechanistic explanation 
of how this is possible (Alizon et al., 2013). Explanations that help to explain altruism also hold true here. For instance, kin selection may be an important factor in within-host parasite interactions (Buckling and Brockhurst, 2008; Leggett et al., 2014).

Numerous parasites are only virulent within certain hosts and not others. Hence, the host may play a key role in the level of virulence and the outcomes of parasite competition. If a parasite evolved towards a higher level of virulence, it would also have greater impacts on host longevity, reproductive rate, and hence be a greater selective pressure on host evolution. Indeed, in the face of highly virulent parasites, rapid host evolution would be expected towards parasite resistance (immunity) (Pal et al., 2007; Duffy and Sivars-Becker, 2007). Furthermore, parasites adapt to local host populations, such that locally common host genotypes are more susceptible to parasites (Dybdahl and Lively, 1998; Lively and Dybdahl, 2000). This means that parasites will select against common host genotypes, driving host evolution and speciation (Nunn et al., 2004). This description of a host-parasite arms race bears some resemblance to predator-prey relationships. A further argument against the avirulence hypothesis is that a predator does not reduce its kill rate to support the prey population on which it survives (Dawkins and Krebs, 1979). However, perhaps the predator might if prey population extirpation was frequent relative to predator life-histories - synonymous with host death in parasite infections.

Host evolution may be a key factor in the level of parasite virulence. However, if the costs of parasitic virulence to the host are lower than an immune response, the host may choose to tolerate a parasite. Further to this, there are a number of intermediate positions for the host. For instance, hosts may utilise a low cost immune response to greatly reduce the detrimental impacts of a parasite and minimise the damage that could be caused by the immune response, but not utilise a high cost immune response that may be required to kill a parasite (Carval and Ferriere, 2010; Little et al., 2010; Sheldon and Verhulst, 1996).

\subsubsection{Native vs non-native parasites, and host-switching}

There is likely to be regular parasite spillover between host species. For instance, some directly transmitted nematodes of browsing ungulates rely on being eaten by their definitive host, and this may be unlikely if there is significant niche overlap with other browsing ungulates. However, how many of these spillover events result in the parasite being successful within a novel host species is unknown (Colwell et al., 2012). 
Humans have introduced numerous non-native species and their parasites to new areas. This results in novel host-parasite species pairings, which may result in successful parasite infection. After successful parasite infection in a new host, the parasite has the potential to be extremely virulent. This is due to a lack of evolutionary history between host and parasite, hence naïve hosts will not have experience of, or evolved defences against, a potentially potent selective influence (Daszak et al., 2000; Daszak et al., 1999). For instance, the introduced fungus Cryphonectria parasitica, which causes chestnut blight, killed nearly all mature North American chestnuts (Castanea dentata) within 30 years of introduction from Japan (Loo, 2009). It is likely that host-parasite evolution will be rapid in the face of such interactions.

It is important to remember that while alien parasites have the potential to be highly virulent, this does not mean that they will be. Parasites may not be able to infect novel hosts or only have low levels of virulence. This is evidenced by people spreading numerous parasites around the world that have not gone on to infect wildlife populations there.

\subsection{Impact of parasites in free-living wild animals}

In the previous two sections I have discussed the variety of different parasitic types and how the abundance of parasites may vary from host to host. I also discussed how the evolution of virulence is dependent on a number of factors. In this section I use two examples to discuss how parasites may impact on populations, in preparation for my review of parasites and the conservation biology of hosts.

\subsubsection{Rinderpest}

The rinderpest virus was declared extinct in 2011. It was a type of Morbillivirus and was closely related to the measles virus, as well as other species from genus Morbillivirus such as the canine distemper virus. The rinderpest virus was transmitted from host to host within water droplets within an individual's breath. Hence, individuals had to be in close proximity to each other for transmission to occur. Further transmission may have occurred due to the presence of the virus in infected individuals' secretions and excretions (Hyslop St, 1979; Taylor et al., 1995).

Rinderpest predominantly infected even-toed ungulates (Artiodactyla), and outbreaks were a serious threat to domestic cattle stocks. The virus could have a variety of effects and 
was often associated with high levels of mortality. The virus had very different symptoms in different host species. For instance, lesser kudu (Tragelaphus imberbis) often went blind from an infection, but with few other symptoms (Roeder et al., 2013). Domesticated cattle could suffer from very high mortality rates; however, certain types of cattle had very few symptoms. There are reports of Asian steppe oxen, Iraqi Baghdadli cattle, and some indigenous Kenyan cattle having very few symptoms while carrying the virus. Although, this resistance to the parasite was complicated by hosts being differentially impacted depending on which strain of the virus they were infected with (Mukhopadhyay et al., 1999; Normile, 2008; Kock et al., 1999).

Historically, rinderpest may have been causing cattle plagues for many centuries. A large outbreak occurred in domestic cattle in Africa during the 1890s. Although the virus was already present in Africa at the time, probably, a growth in the abundance and density of livestock populations increased the transmission rate leading to the outbreak (Roeder et al., 2013). The virus had many large scale outbreaks within wildlife populations. The most recent of these occurred between 1993 and 1997 in Kenya. Lesser kudu, eland (Tragelaphus oryx), giraffe (Giraffa camelopardalis), African buffalo (Syncerus caffer), and impala (Aepyceros melampus) were severely affected, with population losses of up to $80 \%$. The strain was likely introduced through domestic cattle, on which it had only a minimal impact (Kock et al., 1999).

The rinderpest virus was declared extinct in the wild in 2011. This was due to a coordinated program of vaccination of domestic cattle. It relied on a highly effective vaccine, and innovative use of community-based animal health workers (Roeder et al., 2013; Mukhopadhyay et al., 1999). Rinderpest is an example of a highly virulent parasite causing high levels of mortality in some host species. This caused highly visible population crashes over short time frames, but did not result in the extinction of any of its hosts.

\subsubsection{Chronic infections and the interactive effects of polyparasitism}

It is often difficult to measure the impact on host populations of parasites with low levels of virulence (Irvine, 2006). This may be partly due to the range of different impacts they might have. For instance, gastro-intestinal helminths may directly take resources from the host within the intestine. However, they may also indirectly affect the host, due to the increased costs of immune system up-regulation (Lochmiller and Deerenberg, 2000), and also the costs of increased forage selection to avoid further parasitism, which may ultimately lead to a 
reduction in food intake (Gunn and Irvine, 2003).

The costs of parasites with low virulence may lead to a lower overall resource allocation budget. This has been observed experimentally; where body condition is directly influenced by macroparasite load (Stien et al., 2002; Collyer and Stockwell, 2004; Hughes et al., 2009). The resulting reduction in resources will amplify trade-offs between immunosuppression and other nutrient demanding processes (Nordling et al., 1998; Lochmiller and Deerenberg, 2000). This may ultimately reduce the reproductive effort (Forbes, 1993; Goossens et al., 1997; Albon et al., 2002; Stien et al., 2002) and growth rate of hosts (Forbes et al., 2000; Collyer and Stockwell, 2004). Increases in mortality rates have also been observed due to parasites with low virulence. For instance, death may result from an increased susceptibility to predation (Murray et al., 1997) or decreased over-winter body condition (Gulland, 1992; Collyer and Stockwell, 2004). Chronic parasite infections that have low levels of virulence are often widespread in populations. Hence, they only need to have a small effect on individual fecundity or mortality to affect population performance (Hudson et al., 1998; Irvine, 2006).

Parasitology research has contemplated under what conditions a parasite may regulate the size of a host population. Key to population regulation is that an increasing density of hosts will lead to a higher parasite transmission rate and hence higher parasite abundance (1.2.1). This means that, at the population level, at high host density the parasite burden will be greatest. If this burden is great enough to halt population growth then the population may be said to be regulated by parasites (Anderson and May, 1979; May and Anderson, 1979). To determine whether a parasite can regulate host population size the impact of parasitism on fecundity and mortality must be quantified. In reindeer (Rangifer tarandus) there is evidence that a single species of intestinal helminth can regulate population size. Through a treatment experiment, Albon et al. (2002) showed that the nematode Ostertagia gruehneri detrimentally affected host fecundity and, using computer modelling, predicted the effect was strong enough to regulate host population size.

Individuals are rarely only infected by a single species of parasite. More often, a host is affected by a range of different types of parasite. I discussed earlier how parasite competition may reduce detrimental effects on hosts (1.2.2). However, parasite interactions can also be facilitating (Nunn et al., 2014). For instance, nematode infection suppresses immune system functioning in African buffalo. This is likely to facilitate their own success, however it also increases buffalo susceptibility to bovine tuberculosis (Mycobacterium bovis) (Ezenwa et al., 
2010). Hence, although the virulence of a single species of parasite may be low, the combined impacts of polyparasitism may have an addictive or indeed multiplicative effect (Pullan and Brooker, 2008; Bordes and Morand, 2009). The reduction in host fitness caused by polyparasitism has also been observed in African buffalo. Smaller secondary sexual characteristics and higher immune system investment were found in individuals with a more diverse parasite fauna (Ezenwa and Jolles, 2008).

This section has explored how parasites with low levels of virulence can impact on hosts. The cumulative impact of these small effects over long time frames can result in large effects on host population performance. In this section we have also seen the different way parasites can impact populations. In the next section I will discuss when parasites raise concerns in animal conservation.

\subsection{Parasites \& Conservation Biology}

\subsubsection{Parasites and threatened host species}

Parasites are rarely the sole cause of species decline (Heard et al., 2013). For native species of parasite this is unremarkable, as it would be unlikely for us to observe a rapid shift in the host/parasite arms race. Indeed, it is thought that parasites can only cause host population extinction under specific circumstances. Density-dependent transmission predicts that at low host density the transmission rate, abundance, and impact of parasites will be low, while at high host density the transmission rate, abundance, and impacts of parasites will be high. It is thought that this mechanism ensures the host-parasite arms race is not won by either species, because as host density decreases so does parasite pressure (De Castro and Bolker, 2005) much like predator-prey cycles. However, the key assumptions of this mechanism are that a parasite is directly transmitted and host specific, and there are many examples where this is not the case. For instance where transmission is frequency-dependent (1.2.1). Also, generalist parasites may be maintained at high abundance by a high density reservoir host population. Hence, parasites can be a threat to host populations and be a cause of small population extinction (De Castro and Bolker, 2005), in the same way that a generalist predator may drive species extinction (i.e., apparent competition, MacPhee and Sues, 1999).

Threatened species may be particularly vulnerable to parasites, as many of the causes of the current biodiversity decline also expose hosts to a greater parasite burden (Smith et al., 
2009a). For instance, habitat fragmentation and loss can restrict species dispersal, which in turn increases contact rates and parasite transmission and hence ultimately parasite abundance (Scott, 1988). Habitat fragmentation also often creates more extensive interaction between wildlife and human activity, and in particular farming. Domestic livestock can act as high density reservoir-hosts for parasites of wildlife, leading to a higher parasite abundance within wildlife (Lafferty and Gerber, 2002). Indeed it has been shown that wildlife more closely related to domestic animals are more likely to be threatened by parasitism (Pedersen et al., 2007). Other factors that may cause biodiversity loss and also increase parasite infection risk or parasite burden include environmental pollution. A key worry is that common pollutants weaken the immune system, particularly in juveniles, making them more susceptible to the detrimental impacts of parasitism (Selgrade, 2007). Threatened species are also more likely to suffer from inbreeding. A key effect of inbreeding is increased vulnerability to parasitism, which can ultimately increase mortality rates (Coltman et al., 1999). Parasites, while ordinarily unlikely to cause extinction by themselves, may contribute to extinctions where they impact concurrently with other population pressures. Parasites, therefore, may pose a substantial threat to species survival. This is reflected in the fact that the more endangered a species becomes, the greater the threat of parasitism (Heard et al., 2013).

Conservationists must also be aware of a variety of conservation actions that can increase the susceptibility of individuals and populations to parasitism. Animal translocations, in particular, are situations in which the host-parasite population dynamic may be changed, especially in the relocated (reintroduced) or receiving (restocked) population. For instance, conservation translocations may introduce new parasites to an area, and may also introduce naïve translocated hosts to new parasites. This may be exacerbated by the stress caused by the translocation making individuals more susceptible to parasitism. For instance, in black rhinoceros (Diceros bicornis) stress has been shown to cause $15 \%$ of deaths within the first post-release year (Linklater et al., 2011). Another potential influence of species reintroduction may be the introduction of a new reservoir host which would change the existing host-parasite population dynamics in the area (Cunningham, 1996; Jørgensen, 2014; Sainsbury and Vaughan-Higgins, 2012).

Other conservation management actions have also been investigated for their ability to increase parasite burden. These include supplementary feeding which increases transmission rates between hosts at feeding stations (Wright and Gompper, 2005). Also, predator removal may inflate host population density leading to an inflated parasite density and larger parasite 
outbreaks (Packer et al., 2003; Lafferty, 2004). Similar effects are also seen when species become over-crowded within protected areas (Lebarbenchon et al., 2006).

\subsubsection{Non-native parasites}

Non-native parasites are a particular concern for conservationists, because historically parasites such as the previously discussed rinderpest (1.3.1) have caused highly detrimental epidemics (1.2.3). Non-native parasites are the result of novel species pairings; where a host encounters a novel parasite. It is likely that many parasites have been introduced to naïve populations of hosts through the introduction of non-native hosts. For instance, the invasive non-native grey squirrel (Sciurus carolinensis) introduced the squirrelpox virus to the UK, which has severely impacted native red squirrel (Sciurus vulgaris) populations (Sainsbury et al., 2008). Indeed, the changing distribution of hosts, vectors and parasites in response to climate change is projected to increase the frequency of novel species pairings (Altizer et al., 2013).

An important example of a non-native parasite is Batrachochytrium dendrobatidis. This is a parasitic fungus known to infect a variety of amphibians worldwide, but originally from southern Africa (Weldon et al., 2004). Transmission is directly from host to host via zoospores. These are aquatic and motile, and can survive in water for a number of weeks (Fisher et al., 2009). The fungus often causes enormous increases in adult mortality. However, the fungus also infects juveniles, often without ill effect (Woodhams and Alford, 2005). The detrimental effects of the parasite differ between host species. Indeed, in some species of amphibian, the parasite has low virulence, such that they act as reservoir hosts for the fungus. The fungus can affect a variety of potential hosts including non-amphibians within watercourses, such as crayfish (McMahon et al., 2013). The ability of the parasite to cause very high levels of mortality means outbreaks can be highly detrimental to populations. However, the further effects of a variety of reservoir hosts, within other species and juveniles of the same species, means that the parasite has the ability to cause extinction (Lips et al., 2006). This is due to a maintained high level of transmission despite low population density (De Castro and Bolker, 2005). Indeed, it is thought that the fungus has been the cause of the decline or extinction of 200 species of frogs (Skerratt et al., 2007).

\subsubsection{Parasite control strategies}

A wide variety of different parasite control strategies have been utilised for animal 
conservation in a range of circumstances. Some strategies are outlined here to highlight the range of possible techniques. Parasite control does not need to act directly on individuals, but act on levels of transmission. For instance, the transmission of parasites between livestock and wildlife is often a key concern (Pedersen et al., 2007). Transmission rates have been reduced through the creation of buffer zones between livestock and wildlife, and also by actively vaccinating livestock (Bengis et al., 2002; Pedersen et al., 2007; Smith et al., 2009a).

Habitat may be modified to reduce the abundance of a vector. For example, an increase in tick abundance was thought to be the cause of an outbreak of tick-borne diseases, such as Babesia bicornis, in black rhinoceros in the Ngorongoro crater. Tick abundance may have increased due to a suppression of fire in the area, leading to an increase in grass sward height, and more favourable habitat for ticks. A more intensive fire regime was employed which much reduced tick abundance (Fyumagwa et al., 2007).

For some parasites the removal of dead individuals will help to reduce further transmission. For example, anthrax (Bacillus anthracis) can be spread from carcasses. During an outbreak in Canada, the dispersal of anthrax spores was reduced by the burning of carcasses (Gates et al., 1995).

Combinations of parasite control strategies may also be effective. For instance, the feline leukaemia virus is a generalist parasite known to affect a range of felids. During an outbreak in the critically endangered Iberian lynx (Lynx pardinus), it was found to cause an acute anaemia which often led to death within 6 months. Parasite control consisted of removing infected individuals, vaccinating uninfected individuals, and an active reduction of a reservoir host - the feral cat population. It was assumed these measures contributed to the subsequent diminishing of the outbreak (López et al., 2009).

The direct treatment of parasitic infection in endangered hosts has also been attempted. For instance, the endangered pink pigeon (Columba mayeri) is endemic to the island of Mauritius. A protozoan infection, likely the non-native Trichomonas gallinae, was discovered in a sub-population. The infection showed seemingly low levels of prevalence, with clinical symptoms observed in 4.5\% (23 of 508) of adults. However, a treatment program using medicated water improved survival, in squabs showing no symptoms, from $36 \%$ (28 of 84 ) to $82 \%$ (24 of 30) (Swinnerton et al., 2005).

For the conservation of threatened species, often of primary concern is maximizing population growth. Hence, the effect of parasites on individual lifetime reproductive success is 
of concern. Control strategies have been employed in a range of circumstances, however, strategies rarely address a key issue and a conundrum: parasites may be viewed as a threat to conservation reliant biodiversity, but are also a large and important component of biodiversity themselves. As host-specific parasites will be as threatened as their hosts, under what circumstances parasites should be controlled is a key question. Furthermore, the interactions between parasites and their hosts are complex, having both detrimental, as previously discussed, but also positive effects (Thomas et al., 2000). Indeed it is thought that evolutionarily, parasitism is the first step towards mutualism (Michalakis et al., 1992). How conservation actions can be balanced, to consider both threatened species survival and parasite conservation, is discussed further in Chapter 5.

\subsection{The biology, ecology, and conservation of black rhinoceros}

\subsubsection{The biology and ecology of black rhinoceros}

Black rhinoceros (Diceros bicornis) are a critically endangered megaherbivore in the order Perissodactyla. The Perissodactyla are the odd-toed ungulates, which include the asses, horses, and zebra (Equidae), and tapirs (Tapiridae), alongside the rhinoceroses (Rhinocerotidae). The black rhinoceros is one of five extant rhinoceros species, and is the only member of its genus. There are currently thought to be three extant subspecies of black rhinoceros, D. b. bicornis, D. b. michaeli, and D. b. minor. Another subspecies, D. b. longipes, was recently described as extinct (Emslie 2011), and it is debated whether other undescribed subspecies may also have gone extinct. It is questioned as to whether these four clades represent subspecies, ecotypes, or evolutionary significant units (Rookmaaker, 2005) but they are, nonetheless, the taxonomic basis on which conservation strategies are based.

Black rhinoceros are a long-lived species with individuals in captivity often surviving to $>35$ years old (Estes, 1991). Conservationists have identified three key life stages in black rhinoceros, with the age at which an individual develops into the next stage being highly variable (Law and Linklater, 2014). Individuals are considered calves and dependent on their mother until around 4 years of age. Sub-adults may still associate with their mother, but dependency has ceased. Females are thought to transition from being a sub-adult to an adult at around 7 years of age. However, in some exceptional circumstances females may give birth at $<5$ years old (Law et al., 2013), and in this circumstance conception would be estimated to be at 3.5 years old. Males are only thought to transition to an adult stage at around 8 years old, but 
it is unknown when they may start to breed (Law and Linklater, 2014). Recruitment to a population is often slow, with growth rates of $5 \%$ per year considered moderate to high (Adcock, 2009). Predation is not thought to be a significant factor in the death rate of adult black rhinoceros. However, juveniles are known to succumb to lion predation (Plotz and Linklater, 2009). In addition, black rhinoceros are often seen with missing ears and/or tails, and this may be caused by spotted hyenas (Crocuta crocuta) (Owen-Smith, 1988).

Black rhinoceros are predominantly solitary with over lapping home ranges that may vary greatly in size (reported home ranges from $2.6 \mathrm{~km}^{2}-133 \mathrm{~km}^{2}$, Estes, 1991). Male home ranges typically overlap at their margins but may overlap more substantially when males share critical resources, like water, or a clear dominance hierarchy between the males exist with 'floater', subordinate males present. In comparison, female home ranges often, and substantially, overlap (Owen-Smith, 1988).

Adult black rhinoceros average $1.7 \mathrm{~m}$ tall and weigh between 996 and $1362 \mathrm{~kg}$ (Estes, 1991), and so are classed as a megaherbivore (Owen-Smith, 1988). There are some differences in the size of the sexes, with males approximately $11 \%$ larger than females. In addition, female black rhinoceros have been reported to have longer and thinner horns (Owen-Smith, 1988). However, Pienaar et al. (1991) report a mean anterior horn length of $446 \mathrm{~mm}$ (S.D. +/-101) in males $(n=63)$, and $418 \mathrm{~mm}($ S.D. $+/-107)$ in females $(n=49)$, with a mean basal circumference of $493 \mathrm{~mm}$ in males and $454 \mathrm{~mm}$ in females (anterior and posterior horns are also known as rostral and caudal horns respectively). Anterior horn growth may be faster in juveniles, with horns erupting from the skin at around 5 weeks old, and growing approximately $150 \mathrm{~mm}$ in the first year of life. In young adults, horn growth was measured at $60 \mathrm{~mm}$ per year, and decreased to $37 \mathrm{~mm}$ per year in older individuals. Posterior horn growth may be slower (Pienaar et al., 1991). Rhinoceros horn is made predominantly from keratin and is attached to the skin (Hieronymus et al., 2006).

Black rhinoceros have a prehensile upper lip that is used to grasp and direct food into the mouth. Similar to white rhinoceros (Ceratotherium simum), but unlike the Asian rhinoceros species (Rhinoceros spp. \& Dicerorhinus sp.), black rhinoceros have no incisors or canine teeth. This means the species often cut browse with either the pre-molars or molars, creating a $\sim 45$ degree cut to browsed shoots. Many wildlife trackers in South Africa (pers. obs) view this 45 degree cut as a clear indication, 'sign', of browsing by black rhinoceros.

Black rhinoceros are reasonably strict browsers, with grass usually making up a very 
low proportion of diet $(<5 \%)$ independent of habitat (Owen-Smith, 1988), although higher proportions have been reported (e.g., up to 50\%, Landman et al. 2013). The majority of black rhinoceros diet is made up from forbs, dwarf shrubs, and woody plants. Shoot ends are the preferred part of the plant, however bark stripping does also occur. The preferred browsing range is between 0.5 and $1.2 \mathrm{~m}$ tall, with a maximum browsing height of $\sim 2 \mathrm{~m}$ (Owen-Smith, 1988, Wilson, 2001). Black rhinoceros will also push over trees up to $170 \mathrm{~mm}$ in diameter (Owen-Smith, 1988). Black rhinoceros typically drink daily, but have been known to last for 4-5 days without water (Owen-Smith, 1988). Coprophagy has been observed in black rhinoceros in particular eating wildebeest (Connochaetes taurinus) dung (Klingel and Klingel, 1966). However, this activity may be confined to periods when resources are scarce (Goddard, 1968).

Black rhinoceros commonly defecate within middens. These are piles of dung containing the defecation events of multiple rhinoceros (pers. obs.), and will be reused by the same rhinoceros as there will be a limited number of middens within a home range. Faeces are often kicked and scattered after defecation (Freeman et al., 2014). Middens are an interesting defecation behaviour seen in a range of animal species, thought to be used for territory defence and communication. Interestingly, middens may be a way to restrict parasite spread to specific areas, hence creating a landscape with varying levels of infection potential (Smith et al., 2009b, Loker and Hofkin, 2015). Black rhinoceros also regularly wallow in mud, which is thought to cool individuals, and also help with parasite defence and removal, particularly as the mud dries and peels from the skin (Bracke, 2011).

Black rhinoceros size has a number of implications in comparison to other mammalian herbivores. For instance, during digestion gut retention times are longer due to a longer overall gut length, which leads to more cell-wall digestion. However, hindgut fermenters like black rhinoceros have broadly less efficient digestion in comparison to fore-gut fermenters. Black rhinoceros compensate for this by ingesting large quantities of material, with a faster throughput due to no secondary mastication, sometimes called bulk inefficiency. Indeed, faeces often contain large undigested materials, for instance during fieldwork for this thesis, a $6 \mathrm{~cm}$ acacia thorn was found in a rectal faecal sample. The high energy requirements of large body size and the bulk inefficiency strategy, result in a high proportion of time spent foraging. Indeed, black rhinoceros are thought to be active throughout most of the night and approximately one third of the day (Owen-Smith, 1988). 
Black rhinoceros size may also influence their parasite ecology. Parasite biomass within a host scales with host body size, hence as host mass increases so does parasite biomass. However, this scaling is not linear, as mean parasite biomass is often less than expected in larger bodied hosts compared to smaller hosts (George-Nascimento et al., 2004; Poulin and George-Nascimento, 2007). This is thought to show that larger body size may be an evolutionary mechanism to reduce the effects of parasites. However, maximum parasite biomass within a host species does scale isometrically. Hence for some individuals, the proportion of total biomass that is made up of parasites may be the same for the bumblebee bat (Craseonycteris thonglongyai) as it is for a megaherbivore (Poulin and George-Nascimento, 2007). This is greater than may be expected, as metabolic rate and body size scale at a rate of 0.75 (George-Nascimento et al., 2004). This may mean that, in larger bodied species such as black rhinoceros, parasites may have less effect across a population, but have a greater effect in very heavily parasitized individuals in comparison to smaller bodied species.

\subsubsection{Conservation biology of black rhinoceros}

Black rhinoceros were previously found across much of Africa. Numbers across the continent between 1960 and 1995 declined by an estimated 97\%. This decline ultimately resulted in the extirpation of the species from West Africa, and the extinction of the West African subspecies, Diceros bicornis longipes (Lagrot et al., 2007). Black rhinoceros were also extirpated from countries in Eastern and Southern Africa, including Angola, Botswana, Ethiopia, Malawi, Rwanda, and Zambia. Remnant populations survived in Kenya, Namibia, South Africa, and Tanzania. Numbers have since increased from 2410 in 1995 to 4880 in 2010, and black rhinoceros have been reintroduced to Botswana, Malawi, Swaziland, and Zambia (Emslie, 2012b). Illegal hunting (poaching) was the predominant cause of the catastrophic decline in rhinoceros numbers across Africa. Hunting was motivated largely by the demand for horn from Yemen for dagger handles and from Asia for Chinese traditional medicine. The number of rhinoceros lost to poaching has surged again over the last few years. From 2000 to 2007, on average 15 rhinoceros black and white rhinoceros were known to be poached in South Africa per year. In comparison, 443 black and white rhinoceros were lost on average per year between 2008 and 2013, with 1215 rhinoceros lost in 2014. The high price of rhinoceros horn has attracted international crime syndicates with highly sophisticated poaching techniques, such as using unmarked helicopters and immobilisation darts (Biggs et al., 2013). Nations, non- 
government agencies, and businesses (farms and private reserves) with rhinoceros have adopted an extremely cautious approach to rhinoceros research and management with the extreme threat posed to their rhinoceros and staff.

Historically, the South African population has a rather different trend in comparison to the continent wide situation. In 1930, the population of the predominant ecotype of black rhinoceros in South Africa, Diceros bicornis minor, was estimated at only 110. Since then there has been a steady increase to 1684 by 2010 (Emslie, 2012a). Diceros bicornis minor was known to be present in only two such enclosed populations even in 1960. Through an extraordinary program of translocation and reintroduction from these two source populations, numerous populations have now been established.

Wildlife conservation in South Africa is dominated by 'fortress' style conservation, with wildlife kept in strictly designated, fenced, private or public wildlife reserves. The current aim of conservation programs is to maximise black rhinoceros meta-population performance. Keeping populations well below carrying capacity is assumed to increase population growth rate, and this is achieved by regular translocation and the creation of new populations. There may be a number of issues with this strategy. For instance, the assumption that density has an impact on population performance needs verification (Strong, 1986). For example, browsing pressure in low density populations may be too low, allowing preferred species to escape and grow to a non-preferred height.

Many black rhinoceros populations in South Africa are too small to be sustained longterm. For instance in $\sim 2006,65 \%$ of Diceros bicornis minor populations had less than 20 individuals (Adcock, 2009). Hence, this artificial metapopulation needs regular and long-term management through translocation and reinforcement to reduce inbreeding and genetic drift. Unfortunately, translocation success in black rhinoceros is moderate. In an analysis of translocations from 1981 to 2005 , when establishing a new population $8 \%$ of individuals died within the first year, while when reinforcing a population $13.4 \%$ died within the first year. The majority of deaths came after release, with $<2 \%$ of mortality being associated with the translocation itself (Linklater et al., 2011; Linklater et al., 2012). Hence, alternative methods for increasing metapopulation performance, and improving translocation success may be useful to assist species survival.

The black rhinoceros has been listed on Appendix One of the Convention on International Trade in Endangered Species of Wild Fauna and Flora (CITES) since 1976 
(Western and Vigne, 1985). In an effort to reduce poaching a number of individuals have called for a legal trade in rhinoceros horn (Di Minin et al., 2015; Ferreira et al., 2014). It is thought that rhinoceros could be farmed sustainably for their horn, particularly as horn grows back at a predictable rate. The legal trade would help fulfil demand and it is hoped this would incentivise a crackdown on the illegal trade, the market price would be reduced by the increased supply, and taxes raised from the sale could go towards the protection of wild populations.

A key factor in the history of the study species is that the Diceros bicornis minor populations studied within this thesis have been through a severe bottleneck, geographically and in population size. Parasites that could not persist within the small population size, or the geographic area of the refugial population, for instance due to a lack of intermediate host in that area, may have gone extinct. This may have eliminated much parasite diversity (Altizer et al., 2007). There may also be unintended consequences from capture methods that increase the parasite bottleneck at translocations (see Appendix 1). Substantial genetic variation in both host and parasite populations may also have been lost as a result of the translocation bottleneck (Anderson-Lederer et al., 2012). Furthermore, each subsequent population that has been created has represented a further population and genetic bottleneck for both host and parasite.

\subsubsection{The populations studied in this thesis}

The populations I studied were found within three provinces in South Africa Limpopo, KwaZulu-Natal and the Eastern Cape. Limpopo is the northern most province in South Africa, bordering Zimbabwe and the Limpopo River to the north and Pretoria to the South. Ecological carrying capacity (as measured by Adcock, 2014) for black rhinoceros were low in the reserves studied here in comparison to the other provinces. KwaZulu-Natal is the south-eastern province of South Africa. It borders Mozambique and Swaziland to the north, and has a long coastline to the east. It was the province with the highest rainfall in this study. Ecological carrying capacity varied greatly between populations. It is here that the two remnant and source populations of Diceros bicornis minor are found. The Eastern Cape of South Africa is the south-central province centred on the coastal city of Port Elizabeth. Habitat here is dominated by the distinctive Eastern Cape subtropical thicket (Kerley et al., 1995). Although total rainfall here was lower than in other provinces, it was less seasonal. Ecological carrying capacity for black rhinoceros was generally highest in the Eastern Cape.

The populations in this study vary widely in terms of their population growth rate, age 
and the size of their founder population (release cohort). The oldest of the newly created populations studied was established in 1965, while the youngest was established in 2009. In general, I favoured recruiting older populations into the study. This was to decrease the likelihood that influences on parasite abundance from reintroduction (e.g. stress), or the source population, would influence the current population. The mean population age at the time of fieldwork was 16.5 years (the source populations were excluded in this calculation) and only one population that was younger than five years old was investigated. The smallest release cohort size for a population was six and the largest thirty.

I decided that two of the wildlife reserves studied could be reasonably split into more than one rhinoceros population. This decision was made based on consultation with reserve managers and only where hard evidence suggested that migration between the proposed populations was minimal to non-existent. In these two cases, geographic (a major river) and anthropogenic (road) barriers prevented movement.

The populations are not specifically named or mapped here because of requests for anonymity from game reserves for reasons of rhinoceros security and staff safety. This was due to the current high rate of poaching in South Africa. I can, however, provide this information if requested from confirmed legitimate sources.

\subsection{Macroparasites of black rhinoceros}

\subsubsection{Previous work on the parasites of black rhinoceros}

The first consolidation of knowledge on the parasites of black rhinoceros was by Zumpt (1964). This paper succinctly summed the knowledge of the time. The most recent literature has included an up-to-date checklist of all the parasites of black rhinoceros (Penzhorn et al., 1994). A wide variety of different species are parasites of black rhinoceros. These include 33 different species of tick (Amblyomma spp, Cosmiomma sp, Dermacentor sp, Haemaphysalis sp, Hyalomma spp, Rhicephalus spp), of which 3 or 4 are thought to highly prefer black rhinoceros as a host. Numerous other arthropods may also infect black rhinoceros. These include two species of stomach bot fly, Gyrostigma rhinocerontis and Gyrostigma conjungens (Evenhuis, 2012). The latter has not been seen since 1961, and if extinct would leave $G$. rhinocerontis as the only surviving member of its genus (Cogley, 1990). There are also biting flies, such as Rhinomusca dutoiti, which are host-specific to rhino; laying their eggs only in 
rhinoceros dung (Penzhorn et al., 1994). Oxpeckers (Buphagidae) will eat a variety of ticks on black rhinoceros however they may also be viewed as parasitic, as they also often feed on blood at open wounds and mechanically keep the wounds open. The balance of these behaviours, and indeed other services, determines whether they are parasitic or mutualistic (Plotz, 2014).

There are a number of blood piroplasms of black rhinoceros, such as Babesia and Theileria, and trypanosomes. These blood parasites have been identified as a potential cause of mortality during the translocation and reintroduction of black rhinoceros (Brocklesby, 1965; Clausen, 1981; Keep, 1970; Mihok et al., 1995; Mihok et al., 1992; Nijhof et al., 2003; Zimmermann, 2009). The filarial nematode, Stephanofilaria dinniki, has been investigated more than most other rhinoceros parasites. The literature focuses on the pathology of this parasite, which causes large and recognisable lesions along the chest and flanks of black rhinoceros (Boomker et al., 1995; Hitchins and Keep, 1970; Kock and Kock, 1990; Tremlett, 1964). This host-specific parasite may infect all adult black rhinoceros within a population, but is restricted to specific areas in eastern South Africa and Kenya (Plotz, 2014). It was first discovered in 1960 (Schulz and Kluge, 1960) and classified in 1964 (Round, 1964); however, the intermediate host of the parasite has not yet been determined. Also, a single species of trematode has been found to infect black rhinoceros, Brumptia bicaudatum (syn. B. bicaudata), although this species also infects African elephant (Loxodonta africana). A range of parasites live in the intestine of black rhinoceros. For instance, seven species of strongyle (Strongylida) nematodes have been identified that infect South African black rhinoceros, while other nematodes include Oxyuris sp. and Probstmayria sp. (Knapp et al., 1997; Penzhorn et al., 1994).

\subsubsection{Parasites studied in this thesis}

To my knowledge, the non-invasive assessment of parasite abundance within freeliving wild black rhinoceros had never been previously attempted. Here I focused on two commonly occurring parasites of black rhinoceros that can be studied using faecal egg counts, strongyle nematodes and a cestode, Anoplocephala sp. The life cycles of these groups have not been directly assessed but we can use similar species in equids to infer their life cycle.

Strongyles (Strongylida) are a directly transmitted parasite group. The eggs develop within faeces into larvae. Numerous factors may influence the survival of the free-living stages of strongyles, such as temperature and moisture, and they may survive for some months. To 
infect a new host the larvae migrate to vegetation and are ingested as the host feeds (Anderson, 1992). Strongyles undergo a period of development in the intestinal wall or other tissues, before returning to the intestine to feed on the mucosa of the intestinal wall (Schmidt and Roberts, 2005). The strongyle nematodes are known to reduce host resources within equids, with juveniles being most at risk from symptoms such as diarrhoea, colic and hypoproteinaemia (Zajac and Conboy, 2006). Indeed, heavy infections of Strongylus vulgaris within domestic horses (Equus ferus caballus) can cause high levels of mortality in juveniles (McCraw and Slocombe, 1976).

Seven species of strongyle nematode have been identified that infect black rhinoceros in South Africa, Khalilia rhinocerotis and six species from the genus Kiluluma. However, the validity of the species within Kiluluma is in debate. For instance, Knapp et al. (1997) recently found only three species of strongyle nematode in autopsied black rhinoceros. These were Kiluluma goodeyi, Kiluluma magna, and Kiluluma rhinocerotis, and were found at mean intensity within a host of 1310, 5420, and 18490 individuals respectively. Strongyle species cannot be individually determined from egg morphology alone.

Anoplocephala gigantea (Anoplocephalidae) is the only cestode known to infect black rhinoceros in South Africa. Eggs identified to the genus Anoplocephala were identified in this thesis. They were assumed to be A. gigantea, but were not confirmed as this species. This species has been previously identified from white rhinoceros as well (Penzhorn et al., 1994), although evidence from this thesis suggests the populations infecting the different rhinoceros species do not interbreed (see 6.2). Anoplocephala spp. are known to have an indirect life cycle. After defecation, eggs are eaten by an intermediate host, an oribatid mite. Mites then crawl onto vegetation and are eaten by black rhinoceros. After ingestion the tapeworm attaches itself to the intestine wall. A. gigantea scolexes (the part of the tapeworm which attaches to the intestinal wall) were abundant in autopsied black rhinoceros, with 1698 individual tapeworms estimated within a single host (Knapp et al., 1997). A. gigantea is a large tapeworm as its Latin names suggests. Tapeworms in this family have been known to grow up to $6 \mathrm{~m}$ in length (Schmidt and Roberts, 2005). However, adult tapeworms vary greatly in length. As with all tapeworms, A.gigantea does not feed directly on the host but absorbs nutrients from the gut. Hence, their ultimate impacts on their hosts may not be associated with any disease but instead a reduction in absorbed nutrients. However, the attachment by Anoplocephala spp. to the intestinal wall has been known to cause harm in the form of ulceration. Within equids this can lead to intussusception, colic, and potentially mortality (Williamson et al., 1997; Zajac and 
Conboy, 2006; Pavone et al., 2010). However this species may be atypical for the Anoplocephalidae, as a wide variety of differing levels of virulence has been observed between different species within the family (Narsapur 1988).

Oxyuris karamoja is a pinworm known to live within black rhinoceros, and has been recorded at an intensity of 250 individuals per infected host (Knapp et al., 1997). Adult pinworms are thought to crawl out from the anus, laying eggs around the anus. This may cause the host to scratch the anus on woody vegetation or the like, transferring and spreading eggs, with eggs ultimately being either inhaled or ingested by the host species (Noble and Noble, 1982). This method of transmission means eggs may not be found in faeces, but may be found on anaesthetised individuals using the Scotch Tape test (see Chapter 4). Within their host, Oxyuris larvae are known to feed on the mucosa of the intestinal wall which may have some overall effects on host condition, but the virulence of adults is thought to be low (Schmidt and Roberts, 2005; Reinemeyer and Nielsen, 2014). Individuals have been recorded up to $68 \mathrm{~mm}$ in length, although other members of the genus may measure up to $150 \mathrm{~mm}$ in length (Baylis, 1939; Bowman, 2013).

Little is known about the life cycles of the host specific parasites mentioned above. Instead life-cycles are inferred from similar species. Of interest is to debate how parasites specific to black rhinoceros maximise their chances of re-infecting black rhinoceros rather than other ungulates, especially since populations of the critically endangered rhinoceros can be at very low densities. For instance, (as previously discussed in 1.5.1) middens may be a method of parasite avoidance, and hosts may avoid browsing in close vicinity to them. Hence, infective stages may migrate away from middens, either by controlling the behaviour of intermediate hosts, or by the active migration of free-living stages. Also, if a parasite relies on ingestion by the host, they may focus their migration towards browse species and sizes that are preferred by black rhinoceros. Furthermore, they may migrate to parts of a plant that are more likely to be eaten by rhinoceros, such as thicker woody shoots, also less likely to be browsed by other ungulates. Indeed, when hosts are rare it has been predicted that free-living stages of directlytransmitted parasites will first move to actively seek hosts, and then revert to a sedentary tactic to ambush hosts (Fenton and Rands, 2004).

\subsubsection{The quantification of parasites in black rhinoceros}

Knapp et al. (1997) for the first time attempted to quantify the abundance of parasites 
within black rhinoceros. They found a variety of parasite species within autopsied black rhinoceros. In particular, they found the nematode Probstmayria vivipara in enormous quantities (17.9 million individuals within a single animal). However this small pinworm, measuring between 1.7 and $3.3 \mathrm{~mm}$ in length, has only minimal effects on its host (Smith, 1979). Knapp et al. (1997) is a fascinating study but only managed to collect from two black rhinoceros in South Africa. This small sample size reflects one of the difficulties with studying the parasites of black rhinoceros - autopsies on this critically endangered megaherbivore must be opportunistic and are rarely possible.

\section{Faecal egg counts as a measure of parasite infection intensity}

Faecal egg counts (FECs) are a measure of the density of eggs within faeces. They are useful as they have been shown to remain stable from day-to-day, and do not change dependent on the time of day due to circadian rhythms (Döpfer et al., 2004; Nielsen et al., 2006; Rinaldi et al., 2009; Becher et al., 2010; Carstensen et al., 2013; Wood et al., 2013).

FECs have been used for two primary purposes - to identify whether an individual is infected with a parasite species, and to identify the intensity of infection within an individual. A number of studies have assessed whether FECs are useful in detecting whether an individual is infected with a parasite. Many of these studies have concluded that FECs are not useful for this purpose due to high levels of false negative results (Proudman and Edwards 1992; Nilsson et al., 1995; Meana et al., 1998; Williamson et al., 1998; Traversa et al., 2008; Nielsen 2016). In comparison, FECs have been shown to be a reliable measure of the intensity of infection in numerous studies (e.g. Swarnkar et al., 2000; Sinniah, 1982; Sithithaworn et al., 1991; Seivwright et al., 2004; Daş et al., 2011; Rinaldi et al., 2009). However, there is important variation between host species in the reliability of the technique. Table 1.1 shows studies that have investigated the relationship in a range of host species. 
Table 1.1 Studies investigating the correlation between FECs and intestinal parasite abundance in sheep (Ovis aries), goats (Capra aegagrus hircus), cows (Bos taurus), moose (Alces alces), and horses (Equus caballus). The correlation coefficient is given if reported.

\begin{tabular}{|c|c|c|c|}
\hline Ref. & Host & Parasite group tested & Correlation? \\
\hline (1) & Sheep & Strongylida & Significant \\
\hline$(2)$ & Sheep & Strongylida & $r=0.16-0.74$ \\
\hline (3) & Sheep & Strongylida & $\mathrm{r}=0.83$ \\
\hline (4) & Sheep & Strongylida & Significant \\
\hline (5) & Sheep \& Goats & Strongylida & $\mathrm{r}=0.62$ \\
\hline (6) & Goats & Strongylida & $\mathrm{r}=0.6$ \\
\hline (7) & Goats & Strongylida & $r=0.87-0.9$ \\
\hline (8) & Cows & Trematoda & Significant \\
\hline (9) & Cows & Trematoda & Significant \\
\hline$(10)$ & Cows & Strongylida & Not significant \\
\hline$(11)$ & Cows & Strongylida & Significant in younger animals $(<2$ years $)$ \\
\hline$(12)$ & Cows & Strongylida & Not significant in adult animals \\
\hline (13) & Cows & Strongylida & Significant in younger animals \\
\hline (14) & Cows & Strongylida & Significant in younger animals \\
\hline$(15)$ & Moose & Strongylida & $\mathrm{r}=0.42$ \\
\hline (16) & Horse & Strongylida & $\mathrm{r}<0.30$ \\
\hline (17) & Horse & Cestode & $\mathrm{r}=0.71$ \\
\hline (18) & Horse & Cestode & $\mathrm{r}=0.33-0.37$ \\
\hline (19) & Horse & Cestode & Not significant \\
\hline$(20)$ & Horse & Cestode & Not significant \\
\hline
\end{tabular}

(1) Swarnkar et al. 2000; (2) McKenna 1981; (3) Roberts and Swan 1981; (4) Stear et al. 1995;

(5) Cabaret et al. 1998; (6) Rinaldi et al. 2009; (7) Chiejina et al. 2002 (8) Rieu et al. 2007; (9) Mage and Dorchies 1998; (10) Brunsdon 1971; (11) Bryan and Kerr 1989; (12) Michel 1968; (13) Shaw et al. 1998; (14) Ploeger et al 1994; (15) Davidson et al. 2015; (16) Nielsen et al., 2010a; (17) Kjær et al. 2007; (18) Williamson et al. 1998; (19) Proudman and Edwards 1992; (20) Nilsson et al. 1995. 
FECs have been used as an index of intestinal parasite abundance in a range of studies investigating the parasites of free-living populations of large mammals. These include a variety of even-toed ungulates such as buffalo, muskox (Ovibos moschatus) and moose, as well as both Asian elephant (Elephas maximus) and Savanna elephant (Loxodonta africana) (Schultz et al., 1994; Ezenwa 2003; Ezenwa and Jolles 2008; Thurber et al., 2011; Davidson et al., 2014; Davidson et al., 2015; Lynsdale et al., 2015; Debeffe et al., 2016). However, whether they are appropriate in species where the relationship between intestinal parasite intensity and FEC has not been tested is debateable. In Table 1.1, studies show a variety of results when testing the relationship in predominantly domesticated host species. These show that in sheep FECs are a reliable indicator of strongyle infection intensity. However, in cows the strength of the correlation between FECs and internal parasite load is mixed. Indeed, it appears that the relationship for strongyles may become poorer in older individual hosts.

The link between FEC and internal parasite intensity in black rhinoceros has not been assessed. The domestic horse is another odd-toed ungulate, a hindgut fermenter, and also harbours both strongyles nematodes and Anoplocephala sp. Hence, studies in this host may be useful to infer whether FECs in black rhinoceros will be useful. Unfortunately, only a single study has tested the reliability of FECs at predicting strongyle abundance within domestic horses (Nielsen et al. 2010a). For Anoplocephala perfoliata, a cestode of equids, studies have shown both good and poor relationships (Table 1.1).

There is clear variation between different studies when testing the relationship, and a lack of a clear consensus. Exploring possible reasons for the variable correlation between faecal egg counts and parasite intensity is worthwhile in understanding whether FECs will be useful in black rhinoceros.

\section{Potential sources of FEC variability: Faecal volume and consistency}

FECs are a measure of egg density within faeces. Sources of variability in the density of eggs within faeces may come from a range of sources. For instance, if an individual varies in the total volume of faeces defecated this will alter the egg density recorded dependent on the faecal volume. It is interesting to note that cows are unique among tested animals (in Table 1.1) in that their faecal consistency is closer to liquid then other tested ungulates. Further studies investigating how this may affect the egg density estimate may be useful. 


\section{Potential sources of FEC variability: Poor methodologies}

Methodologies for sampling and processing are known to affect FECs and may be a cause of false negative results. For instance, due to between-bolus variability it is recommended that samples are aggregated and homogenised from multiple boluses per individual (Denwood et al., 2012). Another known source of error comes from sample storage techniques. For instance, freezing, fixing in formalin, and a delay in sample processing have all been shown to affect greatly the accuracy of FECs (Seivwright et al., 2004; Dacombe et al., 2007; Jagla et al., 2013; Lynsdale et al., 2015). Hence, ideally samples should be processed on site in the field, to negate any issues with sample storage or delays to sample processing. Sample storage techniques are rarely reported in the literature, hence reviewers should request detailed methodologies so that results can be properly judged. When storage methods are reported examples of bad practice are found (Gooderham and Schulte-Hostedde, 2011).

Egg flotation techniques, such as the McMaster technique, are commonly used for FECs and have been shown to be reliable method of egg enumeration (Cringoli et al., 2004; Denwood et al., 2012). For instance, they have been shown to be a minor source of error when compared to the potential noise created by poor sampling methodologies (Denwood et al., 2012).

A further source of error may come from the methodology of the studies used to test the relationship. A number of studies (e.g. Michel 1968; Ploeger et al., 1994) use the exposure rate to a parasite as a direct surrogate measure of intestinal worm abundance. This ignores the potential impact of factors such as the host immune response and intestinal parasite community competition on ultimate infection intensity (see 1.2.1).

\section{Potential sources of FEC variability: Parasite fecundity}

FECs are predicted to be a measure of the number of female parasites within the intestine. If there is variation in the sex-ratio between host individuals, then this will be an immediate source of error, as FECs will not detect the changing numbers of males. Further sources of variation may come from differing fecundity/egg production levels. For instance, high parasite densities result in higher levels of parasite competition. This leads to reduced adult worm size (Gethings et al. 2016). Adult work size is directly related to levels of egg production, with larger individuals producing more eggs (Kuzmina et al. 2012; Gethings et al. 2016). This means that at high parasite density the egg production rate per individual parasite is lower 
(Sithithaworn et al., 1991; Christensen et al., 1995; Roepstorff et al., 1996; Cabaret et al. 1998). Hence, the relationship between FECs and internal parasite intensity may not be linear, but flatten at high densities (Gillespie, 2006; Gooderham and Schulte-Hostedde, 2011). This should be considered during the interpretation of FECs, in particular when fitting linear correlations between FECs and predictor variables (see Chapter 3).

Acquired immunity in the host may also affect worm fecundity. However, this will only occur if the immune response is strong enough to decrease the resources available to female parasites, but not so strong as to kill or expel the parasite. Hence, this is only thought to be common in primary infections, as in secondary infections the host immune response quickly causes the expulsion of the parasite from the host (Onah and Nawa 2000). Heterogeneity in the acquired immune response may be the cause of the more variable link between FEC and parasite intensity in older cows (Claerebout and Vercruysse 2000). Interestingly in goats, despite a known partially effective immune response to their parasites, FECs still showed a strong correlation with worm burden (Chiejina et al. 2002).

\section{Potential sources of FEC variability: The parasite community}

Egg production rate may differ between species. Strongyles with a large body size produce greater numbers of eggs in comparison to smaller strongyles, and this trend appears to be consistent across many nematode species (Skorping et al., 1991; Ractliffe and Lejambre, 1971; Morand, 1996; Kuzmina et al., 2012). Although, smaller nematodes do have shorter prepatency times (the time taken for the parasite to develop into a reproductive stage in the host) (Skorping et al., 1991). The Equidae are parasitized by 64 different species of strongyle, and crucially these strongyles may differ greatly in size. For instance, domestic horses may be parasitized by the large strongyles (subfamily: Strongylinae) and the small strongyles (subfamily: Cyathostominae) (Lichtenfels et al., 2008). Hence, within a typical horse parasite community you may find between 10-20 species of strongyle from a range of sizes (Kuzmina et al., 2012). This means that a high strongyle egg count may be due to either a few large strongyles, or many small strongyles, resulting in an inconsistent relationship between FECs and the internal abundance of parasites (Kuzmina et al., 2012). Importantly, in the only study to test the relationship between FECs and the intensity of infection, large strongyles were found in $82 \%$ of horses, while small strongyles were found in $100 \%$ of horses (Nielsen et al., 2010a). In comparison, FECs for sheep, thought to be a reliable measure of parasite abundance (see 
Table 1.1), have a typical helminth community dominated by just two or three species (Cabaret et al., 2002; Craig et al., 2006). As FECs may represent either multiple small individuals, or few large individuals, FECs may be good at predicting the biomass, rather than the abundance, of strongyle parasites (Ractliffe and Lejambre, 1971; Coadwell and Ward, 1982).

An assessment of the strongyle species size-range, then, may be useful in attempting to predict whether FECs will be a useful measure of parasite abundance with black rhinoceros. Strongyle nematodes that infect black rhinoceros in South Africa come from only two genera Khalilia and Kiluluma. All these strongyles are of similar size, ranging from 11-23 mm in length, with K. magna slightly larger (Knapp et al., 1997). This diversity of sizes is similar to the size range found in sheep, of which the four main species range from $2.5 \mathrm{~mm}$ to $12 \mathrm{~mm}$ in length (Kaufmann, 1996; Cabaret et al., 2002; Craig et al., 2006). It is also low in comparison to a typical horse community that may harbour strongyles within the range 6-39 $\mathrm{mm}$ in length (Kuzmina et al., 2012). Black rhinoceros are a critically endangered long-lived species. This means opportunities for autopsies are rare, and assessing the efficacy of FECs in black rhinoceros was not possible within the scope of this study. However, predictions from first principles do suggest that some factors responsible for FECs being an uncertain predictor of strongyle infection intensity in horses are not present in black rhinoceros.

Here I have debated the potential influences on the variability of FECs. It is clear that there is evidence for a wide variety of different potential influences on the accuracy of FECs. Despite this, for a proportion of host species and their parasites, FECs are a highly useful tool in predicting parasite infection intensity. The sources of variability should be considered during the interpretation of results. In particular, the likelihood of Type II error, a false negative, is increased due to the potential error inherent in the methodology. For this thesis, to utilise FECs in black rhinoceros, it was essential that sampling and methodological techniques were optimised to reduce any further sources of error. In particular, whether a delay in the collection of samples from boluses after deposition was a potential source of sampling error.

\subsection{Objectives and hypotheses}

This thesis is an investigation of the parasite ecology of the critically endangered black rhinoceros, and assessing the importance of parasites for key challenges in the conservation biology of wildlife, such as maximising population performance. The primary aims of this study were to determine the key influences on parasite abundance within black rhinoceros, 
investigate the effects of parasitism on black rhinoceros and how they can be measured, and debate whether parasites should be controlled within threatened host species. Investigating the determinants of parasite abundance is instructive for potential parasite control and parasite conservation, and to determine where hosts may be most at risk from parasitism. Determining the effects of parasitism is important, and vital for debating whether parasites should be controlled in threatened hosts.

The study of a critically endangered (IUCN, 2013) species illustrates best the conservation issues that may arise due to parasites, while studying the parasite ecology of a megaherbivore may reveal important issues specific to the ecology associated with very large body size (Owen-Smith, 1988). I also chose to study both directly and indirectly transmitted parasites, and this was important as factors may influence the different transmission methods in different ways. These three themes: species conservation, megaherbivores, and parasite transmission contributed an opportunity for a novel advance in our understanding of hostparasite systems and their management.

A key practical requirement of this study was developing successful non-invasive sampling techniques to enumerate parasites within black rhinoceros. As highlighted earlier, the techniques well established in other ungulates, especially domestic stock, had not previously been applied to rhinoceros in a scientific or experimental framework. Hence my first data chapter, Chapter 2, focused on trialling and improving methodologies associated with faecal egg counts for black rhinoceros. Non-invasive strategies could thus be used on this elusive wildlife species - one that is rarely seen in the wild. In particular, it allowed me to utilise faecal samples where defecation had not been observed - a key methodological requirement for this study due to the difficulty observing black rhinoceros defecation in the wild. I was able to demonstrate the efficacy of sampling recently deposited dung, and also quantify the amount of sampling necessary for reliable population-level estimates of parasite abundance. Chapter 2 was published in April 2014 in the International Journal for Parasitology: Parasites \& Wildlife (Elsevier, see Appendix 2).

Using the methodological techniques developed in Chapter 2, Chapter 3 investigates what determines the abundance of parasites at a population level. A wide variety of different factors may influence parasite abundance. Influences, such as density-dependent transmission and abiotic factors (e.g. climate), are likely to impact population-level parasite abundance, while biological influences such an individual's predisposition to parasitism are likely to 
impact variation at the individual level. The relationship between host density and macroparasite abundance is rarely tested in free-ranging wildlife, probably because comparable populations of the same host-parasite relationship are seldom available. I was particularly interested in how directly and indirectly transmitted parasites may be impacted differently at a population level by different influences - host density or climate. Others have suggested that host density is unlikely to drive parasite abundance for indirectly transmitted parasites and I wanted to test whether this was true for black rhinoceros. This chapter gives insight into when populations may carry a high parasite burden, as well as offering clues to how to control parasitism. Chapter 3 was published in April, 2015, in Oecologia (Springer, see Appendix 3).

While the previous chapter usefully investigated the predictors of abundance at the population level, numerous predictors of abundance within individual black rhinoceros were not investigated. Hence, in Chapter 4 I investigated the influence of two often cited individual characteristics that may affect parasite abundance: age and sex. This chapter utilised the extraordinary translocation of 39 black rhinoceros. The investigation of individuals also allowed me to investigate the effects of parasitism on black rhinoceros and how they can be measured. I was interested in whether the large populations of parasites expected in large bodied species could have a potential effect on the resources of a long-lived species like black rhinoceros. Estimating resource budgets is difficult, and particularly challenging in rhinoceros due to the practical difficulties of working with a megaherbivore. Hence, I attempted to utilise novel indices that reflect individual resource budgets, such as the size of sexually selected characteristics.

The investigation of the impacts of parasites on the resource budget of rhinoceros, and hence with potential implications on population performance, led me to question whether parasites should be controlled in threatened host species. I was interested in how the threat of parasitism to endangered species could be balanced with parasite conservation aims, particularly as host-specific parasites will be equally as endangered as their hosts. This valuable and interesting conservation conundrum is unresolved and continues to be debated. Hence, in Chapter 5 I debated when and how parasites should be controlled in threatened host species. I discussed the principles guiding any parasite control, and investigated the literature to see if these principles have been guiding management decisions historically. This chapter is a review of the discipline in which I propose a framework for parasite management in conservation contexts depending, especially, on the status of the host. Chapter 5 was published in October 2014, in Bioscience (American Institute of Biological Sciences, see Appendix 4). 
In my final chapter I draw the outcomes of these chapters together to describe why my study is an advance on what had previously been achieved. In particular, I discuss whether the thesis was successful in the ambitious proposition of identifying the key determinants of parasitism within individuals and populations of black rhinoceros, for both directly and indirectly transmitted parasites. I also build an argument for parasite conservation as an under represented field, worthy of further research. Finally I discuss potential future directions of study and key influences that I was not able to test, such as the influence of genetics on individual parasite abundance. 



\section{Chapter 2: Reducing sampling error in faecal egg counts from black rhinoceros (Diceros bicornis)}

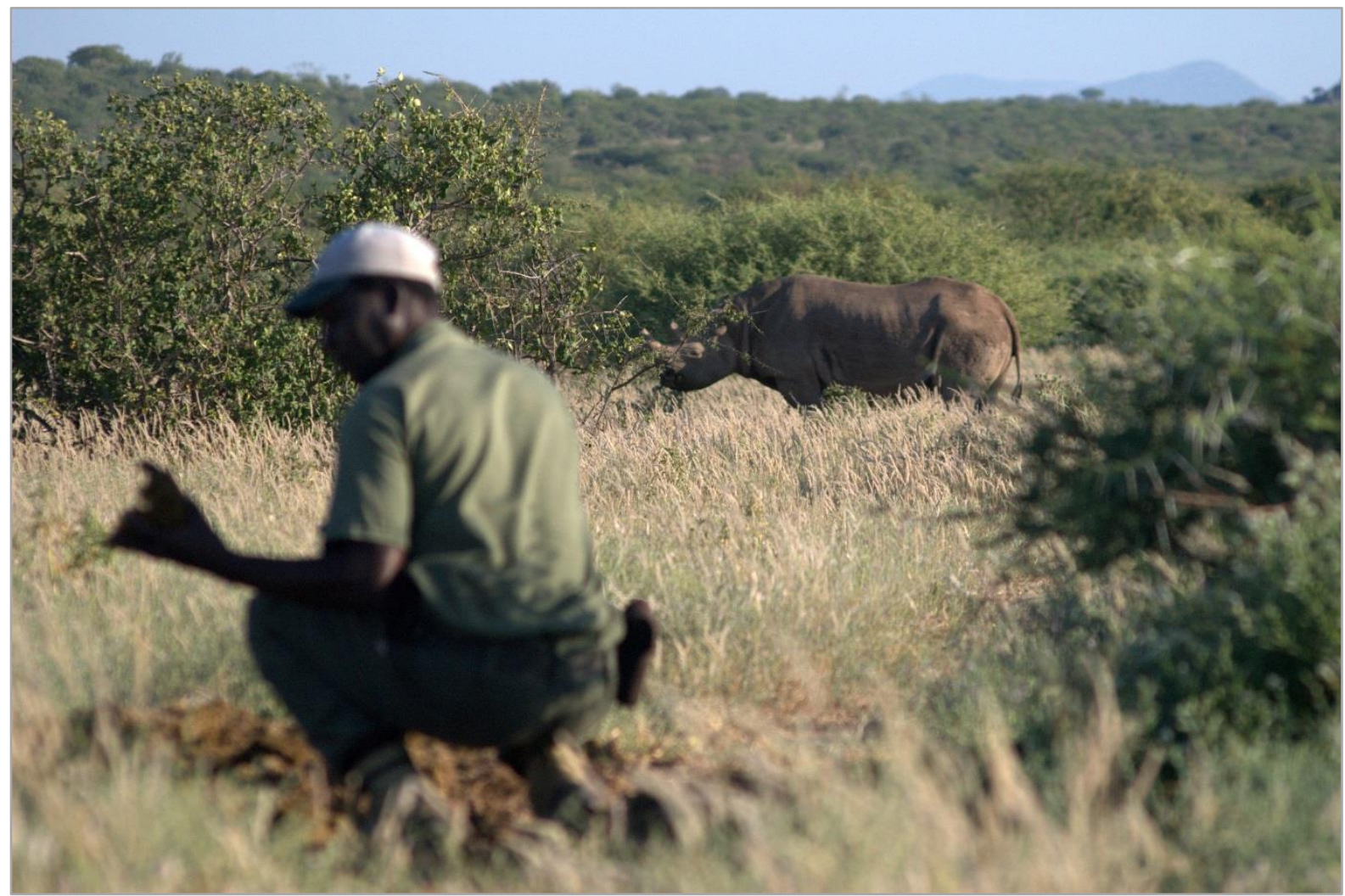

Ranger investigating freshly deposited rhinoceros dung, Maremani Nature Reserve, Feb 2012.

(C) Andrew Stringer

"The flesh of a fat rhinoceros is well tasted, though rather coarse; I do not like the liver."

K.F. Vaughan (1899) 


\subsection{Preface}

In the Introduction, I identified a number of methodological issues that could easily be overcome. For instance, sample analysis can be completed in the field to overcome sample storage issues, sampling from multiple boluses within the faecal pile should overcome heterogeneity issues, and sampling from a single faecal pile should give a good estimation of internal parasite load for an individual. However, there were a number of issues that could not be as simply addressed. Hence, an evaluation and comparison of different sampling protocols was important. In particular, whether faeces where defecation had not been observed could be used, and how best these may be sampled. Also, an estimation of the sample size needed to capture the variability in parasite abundance across a population, so that sampling effort per population could be optimised (i.e. whether to spend more getting more samples from a single population, or whether time would be better spent getting samples from a new population).

This chapter has been published, Stringer A.P., Smith D., Kerley G.I.H. \& Linklater W.L. 2014. International Journal of Parasitology: Parasites \& Wildlife, 3, 1-5, DOI: 10.1016/j.ijppaw.2013.10.002, and is available in Appendix 2. The text is the final text that was submitted to publishers. Larger additions from the published version for this dissertation are enclosed as footnotes, but small changes have been incorporated into the text.

The research described by this chapter and its publication was led by myself. With the assistance of my supervisor I designed the study and its measures, conducted the majority of fieldwork, and wrote the manuscript. Under my direct training and supervision, Diane Smith investigated the difference between FECs taken from the inside and outside of the same bolus. Her work is clearly referenced throughout. All other samples were collected and analysed by myself. 


\subsection{Abstract}

Faecal egg counts (FECs) are commonly used for the non-invasive assessment of parasite load within hosts. Sources of error, however, have been identified in laboratory techniques and sample storage. Here we focus on sampling error. We test whether a delay in sample collection can affect FECs, and estimate the number of samples needed to reliably assess mean parasite abundance within a host population. Two commonly found parasite eggs in black rhinoceros (Diceros bicornis) dung, strongyle-type nematodes and Anoplocephala sp., were used. We find that collection of dung from the centre of faecal boluses up to six hours after defecation does not affect FECs. More than nine samples were needed to greatly improve confidence intervals of the estimated mean parasite abundance within a host population. These results should improve the cost-effectiveness and efficiency of sampling regimes, and support the usefulness of FECs when used for the non-invasive assessment of parasite abundance in black rhinoceros populations. 


\subsection{Introduction}

Evaluating intestinal helminth infections is important for animal production and animal welfare. Parasites may affect population growth (Irvine, 2006) and so the abundance of parasites within populations and across meta-populations is important for the conservation of threatened species. Macroparasites are usually aggregated within a small proportion of a host population (Poulin, 2007a). Gasbarre et al. (1996) concludes that greater than 15 samples are needed to ensure that $95 \%$ of the time the level of parasite aggregation is accurately estimated. Thus, large sample sizes are often needed to capture accurately the level of parasite abundance within a population.

In conservation management, culling an animal to determine its parasite intensity is rarely an option. Opportunistic autopsies on natural deaths can be useful but may give a biased sample of a population's parasite abundance. For some intestinal parasites, faecal egg counts (FECs) are used as a non-invasive tool to determine the abundance of parasites within a host. The technique is particularly suitable for conservation managers because it allows extensive and intensive non-invasive sampling of individual hosts and populations of hosts. Nevertheless, the technique does have constraints.

A direct and positive relationship between FECs and parasite burdens has been shown in numerous studies (McKenna, 1981; Roberts and Swan, 1981; Sinniah et al., 1981; Sinniah, 1982; Sithithaworn et al., 1991; Seivwright et al., 2004; Rieu et al., 2007; Daş et al., 2011). However, an often cited concern with FECs is that this relationship may not be linear (Gillespie, 2006; Gooderham and Schulte-Hostedde, 2011). For instance, at lower parasite densities a sexratio bias towards female parasites may increase FECs independently of actual parasite population sizes (Poulin, 1997). Conversely, at high parasite densities, parasite ovulation rate may be reduced due to inter-specific competition (Sithithaworn et al., 1991; Christensen et al., 1995; Roepstorff et al., 1996). These patterns of variation must be considered during the interpretation of FECs but are not a source of methodological error.

It is recommended that FECs should only be used when samples have been taken rectally or directly after observed defecation (Zajac and Conboy, 2006). This is because temperature, light levels, and oxygen availability may all be cues for the hatching of directly transmitted parasites post-defecation (Nielsen et al., 2010b). Furthermore, the moisture content of faeces may change rapidly or eggs may be eaten by predators, thereby affecting the eggs per gram of faeces estimate (Anderson and Schad, 1985; Eberhard et al., 2001). These mechanisms may 
also differentially influence the FEC within the dung bolus, such as in the surface layer of dung in comparison to the centre, as the surface layer of dung is more exposed to the environment (Daş et al., 2011).

FECs are frequently used to evaluate the abundance of parasites within a host population. However, they are rarely used with elusive host species of conservation concern such as the black rhinoceros (Diceros bicornis). Known sources of methodological error come from laboratory techniques (Cringoli et al., 2004) and sample storage strategies (Seivwright et al., 2004; Zajac and Conboy, 2006; Dacombe et al., 2007). This paper will focus on potential sources of sampling error, primarily the collection of dung where defecation has not been observed. This paper investigates how sampling at known intervals following defecation, and from different locations within the faecal bolus, affects FECs from black rhinoceros. We also determine the minimum number of samples needed to accurately estimate mean parasite abundance within a population.

\subsection{Materials and methods}

FECs were performed on black rhinoceros dung. Two types of parasite eggs were commonly found, strongyle-type eggs (Strongylida), and a cestode, Anoplocephala sp. There are seven species of strongyle that infect black rhinoceros in South Africa (Kiluluma spp and Khalilia rhinocerotis), and only one cestode, Anoplocephala sp. (Penzhorn et al., 1994; Knapp et al., 1997).

To test for differences between the centre and the surface layer of faecal boluses, 43 fresh boluses were sampled over four two-day sampling periods spread between Apr - Sep in 2011 from a wildlife reserve in the Eastern Cape of South Africa. Fresh faeces ${ }^{*}$ were collected during the early morning (dawn - 10am) from middens located along roads. To reduce the possibility of pseudoreplication a stratified random sampling regime, modified to ensure a minimum of 1 $\mathrm{km}$ between sample sites, was used. Black rhinoceros boluses are 12 to $15 \mathrm{~cm}$ in diameter. A sample of approximately $10 \mathrm{~g}$ of dung was collected from the centre of one complete bolus per dung pile. From the same bolus, another sample of approximately $10 \mathrm{~g}$ of dung was taken from

\footnotetext{
* Black rhinoceros faeces can be easily distinguished from other species based on the size and composition of undigested material in the dung. The age of black rhinoceros faeces can also be estimated, with very fresh faeces being warm, dark green, and clearly wet. As the bolus ages, the surface changes in colour to a light brown, and the surface of the bolus starts to dry with external, 'whispy' strands of partially digested vegetation drying first.
} 
the surface to a maximum depth of $1 \mathrm{~cm}$. Each sample was stored at $4^{\circ} \mathrm{C}$ in a sealed plastic bag, with excess air removed, until analysis (within 4-28 hrs) (Nielsen et al., 2010b). A modified McMaster technique (Zajac and Conboy, 2006) using Sheather's sugar solution was used for the flotation and enumeration of parasite eggs. Four replicate chambers were counted for each sample resulting in an analytical sensitivity of 25 eggs per gram (epg) of faeces per individual. Paired t-tests were used to compare differences between the surface layer and centre of each bolus sampled. The differences between these samples were normally distributed. SPSS (IBM, 2011) was used for all calculations unless otherwise stated ${ }^{\dagger}$.

To test how FECs may change due to a delay in sample collection, freshly deposited boluses $(\mathrm{n}=7)$ were collected from black rhinoceros captured for translocation and reintroduction, and held temporarily in purpose-built enclosures in the same Eastern Cape reserve. Boluses of dung were collected at dawn before the animals' enclosures were cleaned. Boluses were judged freshly defecated if they were still warm. Boluses were placed outside the enclosures and subjected to normal daytime conditions. Approximately $10 \mathrm{~g}$ samples were taken from the centre of each immediately and then at 3 hour intervals up until 9 hours after initial collection. Boluses were reformed after each sampling event. Samples were stored and analysed as previously indicated. Strongyle egg maturity was estimated based on the internal structure of each egg. Morulated eggs (Zajac and Conboy, 2006) or those with no clear internal structure were classified as immature, while any egg where larvae or a pre-larval shape could be identified inside the egg were classified as mature. With time, strongyle eggs were expected to hatch and not be recoverable using the McMaster technique while Anoplocephala sp. eggs were not expected to hatch. Data were normally distributed and Mauchly's sphericity test showed that sphericity could be assumed. We used a repeated-measures ANOVA to test whether FECs and the level of egg maturity changed with time after defecation.

We then investigated how sample size affects the reliability of estimates of mean FEC for a population. Fresh faecal samples were collected from 18 populations of black rhinoceros from across eastern South Africa (see Chapter 3), where parasite abundance, aggregation and the number of faecal samples collected from each vary. Bootstrap $90 \%$ confidence interval estimations for the mean FEC using 2000 replications were calculated using the software

\footnotetext{
${ }^{\dagger}$ Diane Smith was primarily responsible for this section of the study - comparing the inside of the bolus with the surface layer. I designed and demonstrated the methods of collection, sampling, and analysis on the first two-day sampling period, fieldwork and analysis on the following three two-day sampling periods was done by Diane Smith.
} 
"Quantitative Parasitology" (Rózsa et al., 2000) for each population. For each population, the size of these confidence intervals (as percentage distances from the mean) were then plotted against sample size. A multiple regression was then used to test how parasite aggregation, estimated mean abundance, and sample size affected confidence intervals. The level of parasite aggregation within a population was calculated using the corrected moment estimation of $\mathrm{k}$ which reduces the bias caused by small sample size (Gregory and Woolhouse, 1993).

Five populations from the previously mentioned dataset with sample sizes larger than nine were used to further investigate the precision of estimates of mean parasite abundance. First, data points were randomised. Then, after each sampling event, mean parasite abundance was calculated. The percentage distance of this mean from the best estimate of the mean using all data points was then calculated.

\subsection{Results}

FECs from the centre of faecal boluses were significantly higher than from the surface layer of boluses for both parasite groups when all four sampling periods were combined (strongyles: $\mathrm{T}_{42}=6.65, \mathrm{p}<0.001$. Anoplocephala $\mathrm{sp} .: \mathrm{T}_{42}=3.23, \mathrm{p}=0.002$ ). Analysing the data for each sampling period revealed significant differences on three out of four sampling periods for strongyles and two out of four sampling periods for Anoplocephala sp.*

For the time-specific FEC sampling, there was a significant reduction in strongyle FECs over time ( $\mathrm{RM}$ ANOVA, $\mathrm{n}=7, \mathrm{~F}_{3,18}=5.1, \mathrm{p}=0.01$ ), while the percentage of mature eggs increased through time $\left(\mathrm{F}_{3,18}=15.1, \mathrm{p}<0.001\right)$. FECs did not decline until after 6 hours had passed since defecation and initial collection. In a pairwise comparison of time points using a Bonferroni correction, the largest difference was between the $3 \mathrm{~h}$ and $9 \mathrm{~h}$ collection points. Anoplocephala sp. FECs were slightly more variable, and did not change significantly through time $\left(\mathrm{F}_{3,18}=0.07, \mathrm{p}=0.86\right.$, Figure 2.1).

Lower confidence intervals for mean abundance improved as sample size increased for both parasite groups (Figure 2.2). Similarly, upper confidence intervals improved with increasing sample size for strongyles, but not for Anoplocephala sp. (although this trend appears to be driven by a single extreme value). Upper confidence limits were generally much

\footnotetext{
* Data collected by Diane Smith, but re-analysed by myself.
} 
further from the mean. Generally, confidence intervals were much improved when sample size was greater than nine (Figure 2.2).
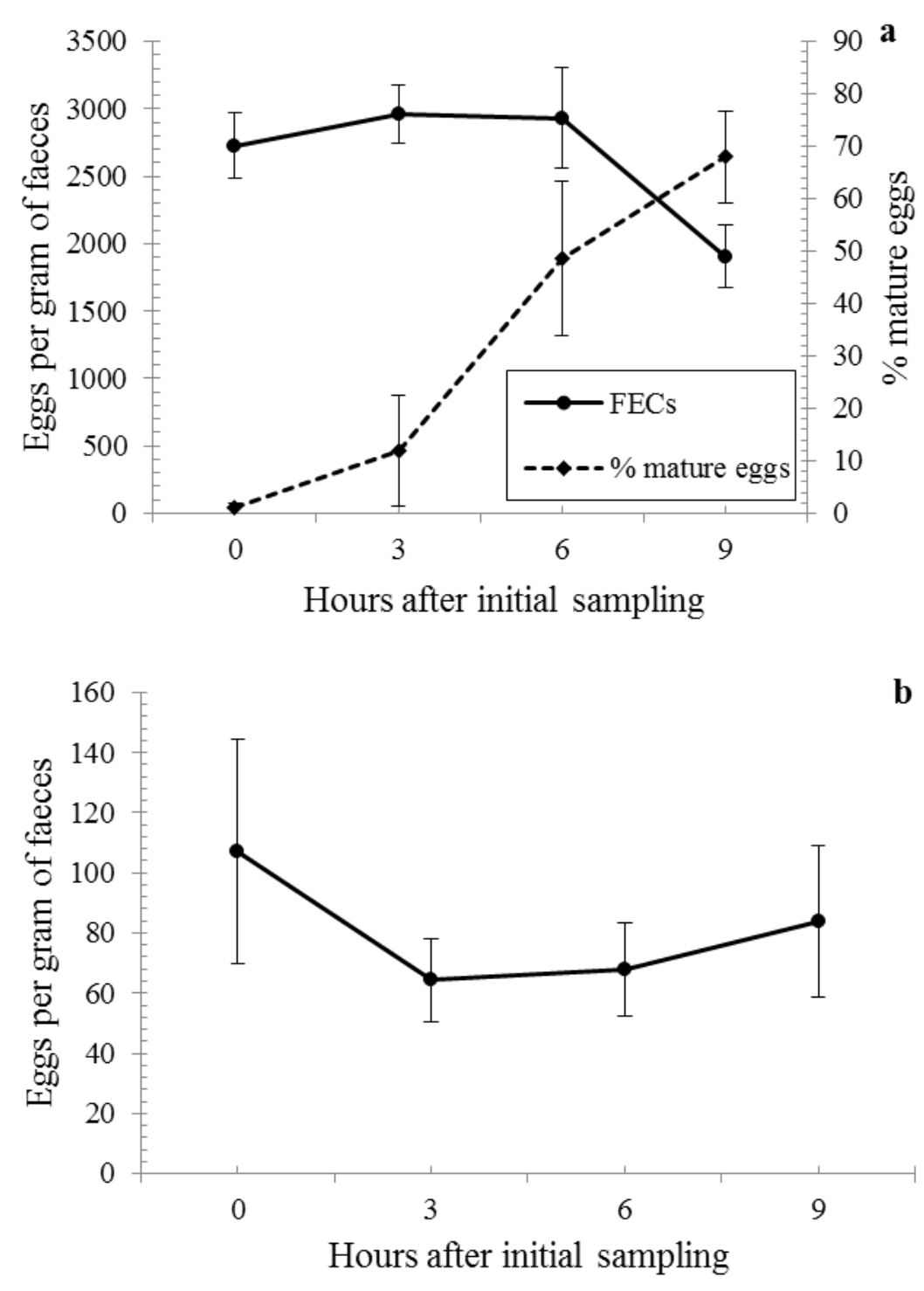

Figure 2.1 a. Mean strongyle FECs and mean percentage of mature eggs, sampled 3, $6 \& 9$ hours after initial collection from captive black rhinoceros. Error bars represent +/- S.E. b. Mean Anoplocephala sp. FECs, sampled 3, $6 \& 9$ hours after initial collection from captive black rhinoceros. Error bars represent +/- S.E.

Further investigation included the impacts of aggregation and calculated mean on the size of confidence intervals. A multiple regression revealed that the size of confidence intervals for strongyles was significantly predicted by parasite aggregation $($ Beta $=-0.57, \mathrm{p}<0.01$ ) and mean abundance $($ Beta $=-0.61, \mathrm{p}<0.01)$ but not sample size $($ Beta $=-0.11, \mathrm{n} . \mathrm{s})$. The overall 
mode fit was $\mathrm{r}^{2}(\operatorname{adj})=0.63$. While, for Anoplocephala sp. confidence intervals were significantly predicted by parasite aggregation (Beta $=-0.82, \mathrm{p}<0.001$ ) but not mean abundance $($ Beta $=-0.04, n . s)$ or sample size $($ Beta $=-0.21, n . s)$. The overall model fit was $\mathrm{r}^{2}$ $(\operatorname{adj})=0.61$.

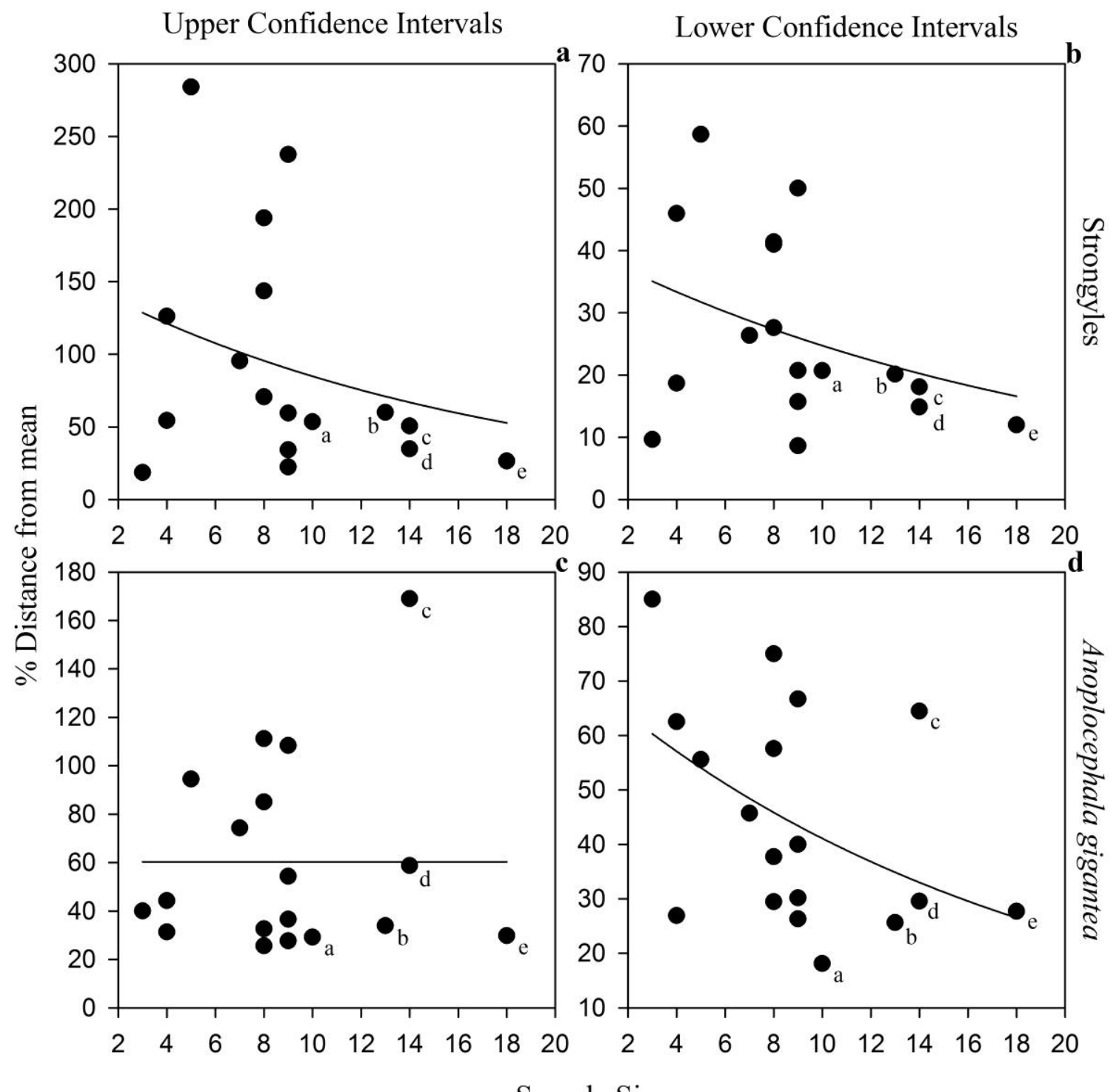

Sample Size

Figure 2.2 The distance from the mean of bootstrap 90\% confidence intervals plotted against sample size for FECs for 18 black rhinoceros populations. Lines represent the fitted negative exponential curve. Five populations are labelled "a-e" that are used in Figure 2.3. a. Strongyle upper confidence intervals. b. Strongyle lower confidence intervals. c. Anoplocephala sp. upper confidence intervals. d. Anoplocephala sp. lower confidence intervals.

Using only those populations with nine or more samples, after six samples, all estimates 
of mean parasite abundance were within $20 \%$ of the best estimate for strongyles (Figure 2.3a). The population that took longest to improve had the lowest mean abundance. While for Anoplocephala sp., after nine samples 4 out of 5 populations' estimates of the mean were within $20 \%$ of the best estimate. The single population that took longer to improve (Figure 2.3b) is the same extreme result seen in Figure 2.2c, and is associated with a high degree of parasite aggregation within that population.
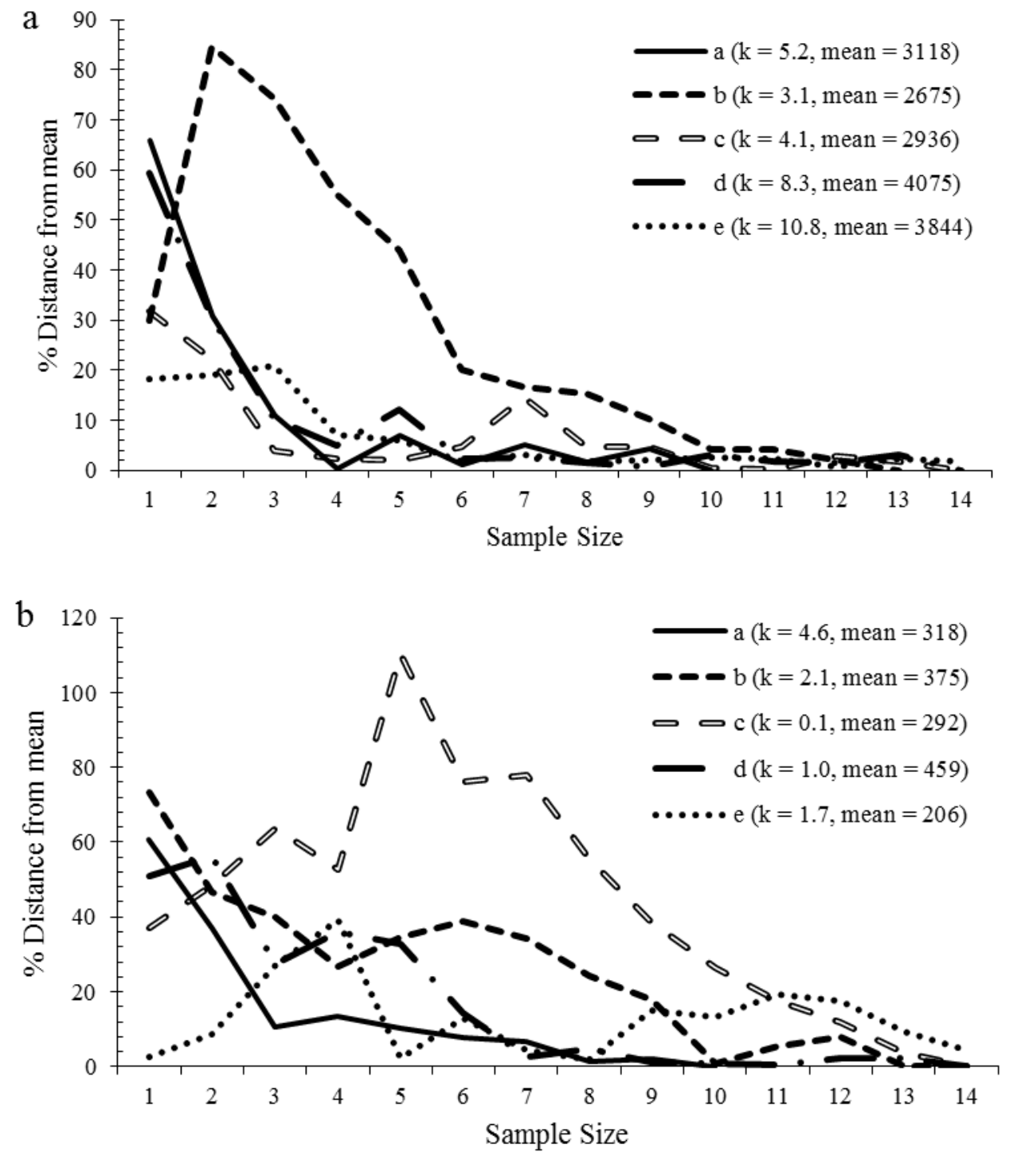

Figure 2.3 The accuracy of the estimated mean after each sampling event is plotted for each reserve with 10 or more samples. The corrected moment estimate of $k$ and the population mean are given in the legend. a. Strongyle nematodes b. Anoplocephala sp.. 


\subsection{Discussion}

Parasite enumeration in free-ranging wildlife is often difficult and time consuming. There are many potential sources of error that may affect FECs. Identifying these sources of error is important so that they can be controlled by experimental design or considered during the interpretation of results.

Overall FECs were lower in faeces collected from the surface layer of boluses in comparison to the centre of boluses, although this trend differed between sampling periods. It is possible that this variation is caused by exposure to environmental conditions and predation, depleting egg density in the surface layer. Sampling from the centre of boluses may be a useful technique for host species with similar sized or larger boluses, such as white rhinoceros (Ceratotherium simum) and African elephant (Loxodonta spp.). In other host ungulate species, with smaller faecal boluses and larger surface area to volume ratios, environmental influences may more quickly penetrate the centre of boluses.

FECs were robust to quite long periods between defecation and sample collection. Strongyle eggs matured during the initial 6-hour period after defecation but this did not affect FECs. Anoplocephala sp. FECs did not decline, however these eggs do not develop into larvae as they are instead eaten by their intermediate host. Hence, the number of eggs eaten by intermediate hosts or predators, or decaying up until 9 hours after defecation was minimal. It must be noted that all FECs were calculated by the wet weight of the sample rather than the dry weight. Hence, it would be expected that as water evaporated from the dung calculated FECs would increase. We did not observe any significant increase in FECs indicating that actual egg numbers may have reduced over time or that evaporation from the centre of dung boluses was minimal ${ }^{\S}$.

A sampling regime that collects samples without observing defecation is only useful if faeces can be identified as being collected within 6 hours of defecation. Here, although the rate at which strongyle eggs mature was variable between samples, the level of egg maturation within the sample could be used to broadly assess the age of faeces. There could be other

\footnotetext{
$\S$ There was a non-significant increase in EPG after 6 and 9 hours for Anoplocephala sp that may have been due to evaporation from the bolus through time.
} 
problems with collecting older samples. For instance, mature eggs may be more difficult to identify (Zajac and Conboy, 2006), although this was not found for the parasite groups studied here. Finally, when defecation is not observed, the accidental collection from the same individual would be possible. A stratified random sampling regime would reduce the chance of this pseudoreplication (Dytham, 2003), but not eliminate it.

Collecting more than nine samples greatly improved confidence intervals for the population mean. However, there was a great deal of statistical noise associated with this relationship. Some of this could be explained by the estimated mean - a smaller mean was associated with larger confidence intervals because our methodology had a resolution of 25 epg. Hence, if egg counts are low then the methodological resolution of egg counts should be increased. This may be done by decreasing the dilution factor or increasing the number of replicates from each sample (Torgerson et al., 2012).

A large amount of variation in the size of confidence intervals was explained by the level of parasite aggregation within a population. Hence, if parasite aggregation is high more samples may be required in order to improve confidence intervals. This reflects one of the disadvantages of reporting mean abundance in parasite studies - the mean is dependent on a few heavily infected individuals and is not accurately indicative of the typical infection across the population of hosts (Rózsa et al., 2000). This was shown in Anoplocephala sp. (Figure 2.3b) where, although the accuracy of estimates of mean parasite abundance was positively associated with sample size, certain populations required many more samples for mean abundance estimates to become accurate. This was likely caused by a few, heavily parasitized individuals.

Depending on the study aims, the mean level of infection need not be determined at all. For instance, Generalised Linear Mixed Models allow for non-normally distributed data and random effects, hence mitigating the need to reduce each population to a single data point (Bolker et al., 2009). The required sample size is then dependent on the study questions whether it is the typical level of infection or the heavily parasitized individuals that are of interest.

These results should reduce the costs and labour of data collection, and increase the usefulness of FECs as a tool for the non-invasive assessment of parasite abundance. Although this study was specific to black rhinoceros, the techniques used here could apply to numerous other host species. These may include other rare or elusive members of the Perissodactyla and 
the Elephantidae. However, studies wishing to use a delayed faecal sampling strategy must independently test the speed at which FECs decline. Quick and easy methods of parasite enumeration will assist conservation managers identify when parasites may be of concern for the conservation of threatened species. 



\section{Chapter 3: Host density drives macroparasite abundance across populations of a critically endangered megaherbivore}

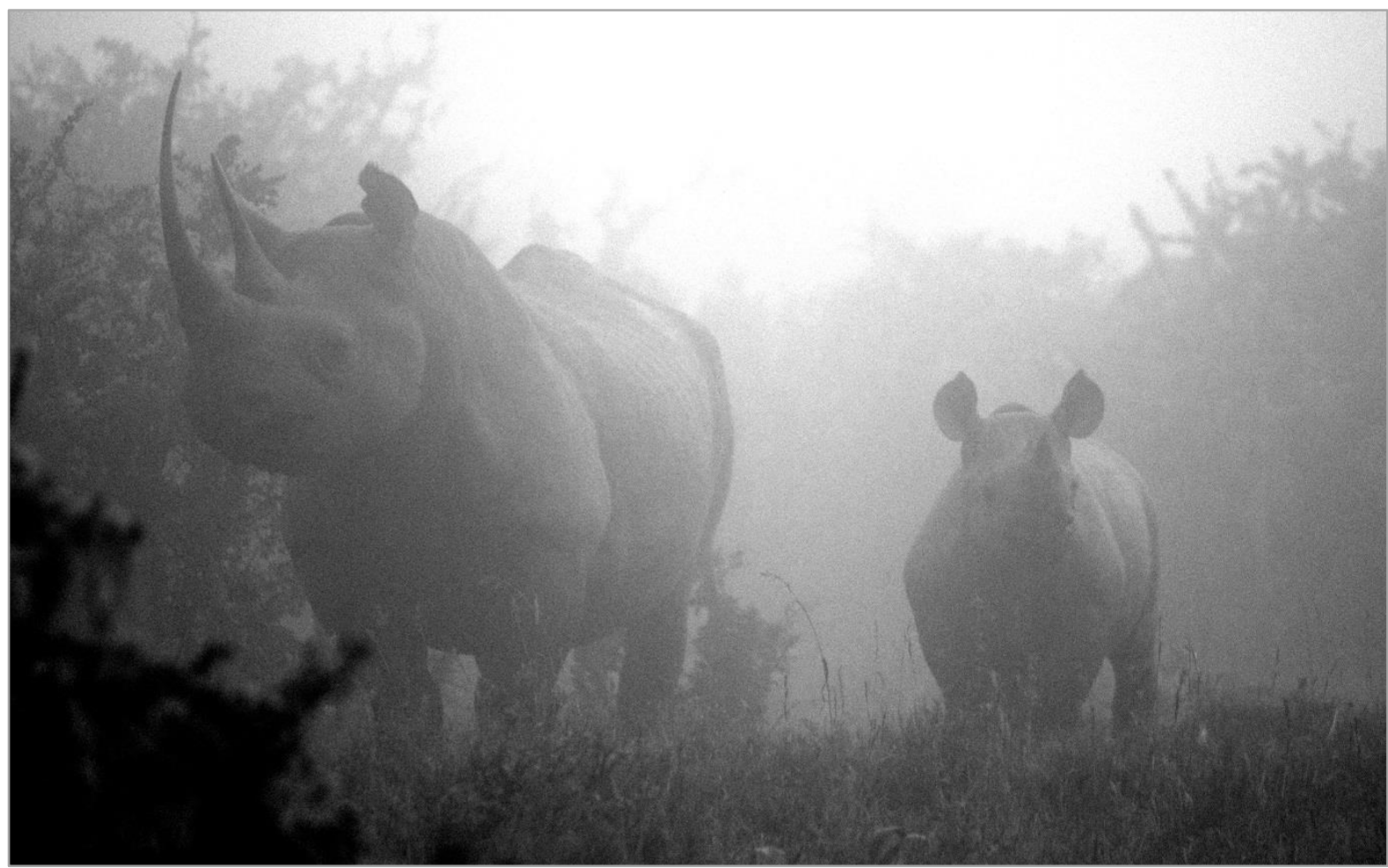

Rhinos in the mist, Eastern Cape, Apr 2011, (C) Andrew Stringer.

$$
\text { "What man dare, I dare; }
$$

Approach thou like rugged Russian bear,

The armed rhinoceros or the Hyrcan tiger,

Taken any shape but that, and my firm nerves

Shall never tremble"

William Shakespeare, Macbeth (1606) act 3, sc.4, 1. 99 


\subsection{Preface}

The previous chapter helped improve methodologies, allowing samples to be collected from faeces where defecation had not been observed. It also allowed fieldwork to be optimised in an attempt to collect sufficient samples from as many populations as possible in the time allowed and under the constraints on population sample-size imposed by the current international rhino-poaching crisis. The following chapter applies these methodological advances to investigate population-level parasite abundance, in an attempt to determine one of the key aims of the thesis - the determinants of parasite abundance on a population level.

This chapter has been published, Stringer A.P. \& Linklater W.L. 2015. Oecologia, 179(1):201-207, DOI: 10.1007/s00442-015-3319-1, and is available in Appendix 3. The text is the final text that was submitted to publishers. Larger additions from the published version for this dissertation are enclosed as footnotes, but small changes have been incorporated into the text. 


\subsection{Abstract}

What determines the abundance of parasites is a central question within epidemiology. Epidemiological models predict that density-dependent transmission has a principal influence on parasite abundance. However, this mechanism is seldom tested in macroparasites, perhaps because multiple, comparable populations of the same host-parasite relationship are rare. We test the influence of a range of factors on parasite abundance across 18 populations of black rhinoceros (Diceros bicornis) in South Africa. Here we show that host density strongly predicts parasite abundance at the population level for both directly and indirectly transmitted parasites. All other models were not supported. The surprising influence of a single key factor, host density, within a complex ecological system demonstrates the validity of simple epidemiological models. Establishing this previously assumed relationship between host density and parasite abundance has major implications for disease control and parasite ecology. For instance, it is central to the idea of population density thresholds for parasitism, below which a parasite would become extinct. Density-dependent transmission is also essential for calculations of the basic reproductive number, and the hypothesis that parasites may regulate host population size. 


\subsection{Introduction}

Epidemiological theory for parasite and disease abundance relies on the assumption of density-dependent transmission (Anderson and May, 1991). High host density is assumed to increase parasite abundance because parasites more easily find a host to invade. Although predicted by numerous epidemiological models (Anderson and May, 1979; May and Anderson, 1979; Anderson and May, 1991; Arneberg et al., 1998) and previously shown for microparasites (Lloyd-Smith et al., 2005), density-dependent transmission has rarely been documented for macroparasites of wildlife. This may be because macroparasite transmission is often more complicated than microparasite transmission due to external free-living life stages, which obscure the mechanism and have led to a number of alternative competing theories (Hudson and Dobson, 1995). Also, for indirectly transmitted parasites, the mechanism is further complicated by the interaction between primary and secondary host density (Stien et al., 2010).

Density-dependent transmission is difficult to test because multiple, isolated, suitably variable, yet comparable populations of the same host-parasite interaction are rarely found. Such real-world experimental field data is needed to further develop epidemiological theory (Diamond, 1986; Anderson and May, 1991; Lloyd-Smith et al., 2005).

Arneberg et al. (1998) showed a positive relationship between the average abundance of parasites in different host species and host species density. However, the demonstration of an inter-specific association is not proof of a population-level ecological mechanism. Variability in host and parasite density through time for single host populations has been used to test the mechanism. A number of studies have shown a general positive relationship (Hudson et al., 1992; Albon et al., 2002; Jansen et al., 2012), however negative relationships have also been reported (Haukisalmi and Henttonen, 1990). It seems difficult to attribute cause where only single populations are used, but multiple influences are considered (Haukisalmi and Henttonen, 1990; Cattadori et al., 2005b). Here we test the influence of host density on parasite abundance by comparing multiple populations of the same host-parasite interaction.

Numerous other mechanisms may act on parasite abundance. For instance, environmental variables may impact the development rate or survival of external free living parasite stages (Mouritsen and Poulin, 2002), or for indirectly transmitted parasites, egg survival (Atkinson et al., 2013). Hence, we also test for the influence of a variety of environmental factors on parasite abundance as predicted by the literature. Likewise, the use of fire, as a form of intermediate disturbance, is likely to have a profound effect on the survival 
of eggs, free living stages, and the abundance of intermediate hosts (Fuentes et al., 2007). Also, the size of a founder population may impact on the genetic diversity of either host or parasite population, potentially affecting the host-parasite interaction. The influence of fire regime and founder population size is also tested here.

Black rhinoceros (Diceros bicornis) are host to numerous parasites including a wide variety of both directly and indirectly transmitted gastrointestinal helminths (Penzhorn et al., 1994). Black rhinoceros populations in South Africa are managed as an artificial metapopulation. Between 1960 and 2005, in eastern South Africa, 442 rhinoceros were reintroduced to reserves to speed species recovery. Remarkably, seventeen out of the eighteen populations of black rhinoceros in this study originated from only two, genetically very similar (AndersonLederer et al., 2012), source populations. Many are still expanding, providing a range of rhinoceros densities that are not determined by their environment. We are able, therefore, to use these populations as different treatments in a fortuitous country-wide field or pseudoexperiment (Diamond, 1986; Kerr et al., 2007). By comparing the abundance of both directly and indirectly transmitted parasites between these different populations, we can test for the influence of a range of environmental and ecological factors.

Faecal egg counts are known to vary from season to season (Baudena et al., 2000, Nalubamba et al., 2012). Also, although non-significant, there has been shown to be some variation in samples taken from different locations within and between faecal boluses (Lynsdale et al., 2015). Hence, sampling regimes must take account of these potential sources of variation and attempt to negate or capture it.

\subsection{Materials and methods}

Fresh faecal samples $(\mathrm{n}=160$, mean samples per population $=8.9$, range $=3-18$, Figure 3.1) from 18 black rhinoceros populations spanning a variety of climatic zones (Table 3.1) in eastern South Africa were collected. The populations studied were all fenced and intensively monitored, hence rhinoceros density could be accurately estimated. Population size ranged between 9 and 200 individuals, within reserves that ranged from 3668 ha to 90000 ha in size. Populations varied greatly in age. All populations had been created at least three years prior to data collection, with a mean age of 16.5 years (range: 3 - 46 years) excluding the two source populations. The majority of the study populations were established from only two source populations from South Africa (which were also included in the study), the solitary exception was created using a Namibian source population. 
To mitigate the effects of season all samples were collected during the wetter summer season (Oct-Apr). The date of sample collection was originally included in the competing set of models and found to have no effect on parasite abundance. Fresh faeces were located along roads and game trails in the early morning (dawn-10am). A sample of approximately $20 \mathrm{~g}$ was taken from the centre of three boluses from the same faecal pile. This was thoroughly mixed, before $2 \times 4 \mathrm{~g}$ samples were extracted for analysis. To reduce the possibility of pseudoreplication a stratified random sampling regime, modified to ensure a minimum of $1 \mathrm{~km}$ between sample sites, was used. Also, local knowledge of rhinoceros home ranges was used to reject samples that were likely to have come from the same individual. ${ }^{* *}$

Table 3.1 Descriptive statistics from the environmental parameters used in candidate models.

\begin{tabular}{lllll}
\hline & Mean & SE & Min & Max \\
\hline Max temperature * $(\mathrm{C})$ & 26.8 & 0.12 & 22.6 & 29.7 \\
Summer mean max temperature $* *(\mathrm{C})$ & 28.8 & 0.14 & 23.8 & 32.4 \\
January min temperature **(C) & 19.1 & 0.12 & 15.4 & 22.2 \\
Min temperature * $(\mathrm{C})$ & 13.8 & 0.14 & 10.1 & 17.0 \\
Humidity * $(\%)$ & 76.9 & 0.28 & 68.6 & 86.4 \\
Rainfall $($ Mean monthly) $*(\mathrm{~mm})$ & 45.9 & 0.81 & 31.0 & 79.6 \\
Summer rainfall ** (mm) & 441.5 & 9.3 & 223.8 & 837.2 \\
\hline
\end{tabular}

*Mean value 2000-2011, ** During season of collection, Summer: Oct-Mar

Faecal samples were stored in anaerobic conditions in a cool box before immediate (2$4 \mathrm{hrs}$ ) analysis, or refrigerated at $4{ }^{\circ} \mathrm{C}$ for no more than $48 \mathrm{hrs}$ before analysis. Faecal egg counts were performed using a modified McMaster technique, using Sheather's sugar solution (Zajac and Conboy, 2006). Quality of samples was ensured by the identification of mature or immature embryonated eggs. Samples deemed too old based on egg maturation ( $>30 \%$ mature) and field observation of faeces were rejected (Chapter 2). Eggs were identified using Zajac and Conboy (2006). Representative photos of all egg types found in a population were taken to confirm identification across populations. Two parasite groups were studied - strongyle-type nematodes (species of strongyle cannot be distinguished by egg morphology alone) and

\footnotetext{
** Tracks, signs, and the size and age of faecal boluses were also used to identify where boluses had come from different individuals, but were geographically close (i.e. $<1 \mathrm{~km}$ apart, see 3.7 ).
} 
Anoplocephala sp. In South Africa, seven species of strongyle have been identified that infect black rhinoceros, while only one cestode has been identified, Anoplocephala gigantea. All of these are thought to be host specific to black rhinoceros (see 6.2). Strongyle nematodes are directly transmitted parasites - eggs develop into free-living stages which crawl onto vegetation and are subsequently eaten. A. gigantea is an indirectly transmitted tapeworm - eggs are eaten by an oribatid mite (oribatida), which are subsequently thought to crawl onto vegetation before being accidentally eaten (Zajac and Conboy, 2006).

Here we use the term abundance as described by Bush et al. (1997), in that we calculated an estimated intensity of infection within each host, but also included non-infected hosts in our analysis. Hence, our results may reflect both the prevalence and the intensity of infection within a population.

An Information-Theoretic approach was used to explain parasite abundance by comparing candidate models. The competing candidate set of models (hypotheses) was developed by consulting the literature (Tables 3.2 and 3.3). Sample size for some populations was very small. Hence we used mixed-models to test the relationship of candidate models to parasite abundance, and either generalized linear mixed-models (GLMM) or linear mixedmodels (LMM) were used (see 3.5). Population could then be specified as a random effect, allowing us to utilise all samples collected, rather than reducing each population to a single mean value. Furthermore, a second-order Akaike Information Criterion $\left(\mathrm{AIC}_{\mathrm{c}}\right)$ was used to compare competing models, which takes into account sample size. For GLMMs, a negative binomial distribution with a log-link was used. In all models the parameters were the same except for the fixed effect. $\mathrm{K}$ relates to the number of parameters in each model (i.e. $\mathrm{k}=3=$ fixed effect + random effect + intercept).

Models with the lowest $\mathrm{AIC}_{\mathrm{c}}$ were deemed to have the strongest support. Model selection was based on the top performing models that represented $95 \%$ of Akaike weights. A base model containing only the random effect was included in the competing set of models to investigate how much information in the data was not being explained by competing models. To avoid overfitting (Zuur et al., 2009), the number of parameters within each model (K) was kept to a minimum by initially including only one fixed effect per model (excluding the base model which had no fixed effects). A suite of models with two fixed effects was then produced that contained the top performing predictor variable plus each of the other parameters in turn. As full models could not be used the information-theoretic approach could not be utilised fully 
in this study. However, we view this study as providing a useful framework which can be improved upon, either with further populations of black rhinoceros, or with species that aren't as rare as black rhinoceros.

SPSS (IBM, 2011) was used for all statistical calculations. Fire regime was estimated based on reserve records. Environmental variables were obtained by the South African Weather Service station closest to the populations (mean distance $27.6 \mathrm{~km}$ ), and varied extensively between populations (Table 3.1).

\subsection{Results}

Table 3.2 Information-theoretic table of candidate models explaining strongyle parasite abundance as a function of host rhinoceros density (rhinoceros.ha ${ }^{-2}$ ).

\begin{tabular}{llllll}
\hline Model/Hypothesis & Source & $K$ & $\mathrm{AIC}_{\mathrm{c}}$ & $\Delta \mathrm{AIC}_{\mathrm{c}}$ & $\omega$ \\
\hline Host density & $(\mathbf{1})$ & $\mathbf{3}$ & $\mathbf{2 6 9 6 . 3 0 5}$ & $\mathbf{0 . 0 0}$ & $\mathbf{1 . 0 0 0}$ \\
Max temperature * & $(2)$ & 3 & 2716.509 & 20.20 & 0.000 \\
January min temperature ** & $(3)$ & 3 & 2717.377 & 21.07 & 0.000 \\
Summer mean max temperature ** & $(4)$ & 3 & 2717.540 & 21.24 & 0.000 \\
Min temperature * & $(2)$ & 3 & 2718.423 & 22.12 & 0.000 \\
Humidity * & $(2)$ & 3 & 2720.264 & 23.96 & 0.000 \\
Fire regime & $(5)$ & 3 & 2721.427 & 25.12 & 0.000 \\
Population Age & & 3 & 2721.172 & 25.87 & 0.000 \\
Rainfall * & $(2)$ & 3 & 2722.378 & 26.07 & 0.000 \\
Founder population Size & $(6)$ & 3 & 2724.379 & 28.07 & 0.000 \\
Summer rainfall ** & $(4)$ & 3 & 2727.266 & 30.96 & 0.000 \\
Base model & & 2 & 2730.649 & 34.34 & 0.000 \\
\hline
\end{tabular}

Models are in descending order from most to least supported based on Akaike second-order Information Criteria $\left(\mathrm{AIC}_{\mathrm{c}}\right)$. The base model, highlighted in grey, included only the randomeffect for population. The confidence set of models, i.e. $>95 \%$ of Akaike weights $(\omega)$, are in bold. $\mathrm{K}$ is the number of parameters in a model. Number in parentheses refers to reference. *Mean value 2000-2011** During season of collection. The set of candidate models differs between parasite groups as they use different transmission methods. (1) Arneberg et al. 1998; (2) Mouritsen and Poulin 2002; (3) Hudson et al. 1992; (4) Haukisalmi and Henttonen 1990; (5) Fuentes et al. 2007; (6) Paterson et al. 1998. 
Table 3.3 Information-theoretic table of candidate models explaining Anoplocephala gigantea abundance as a function of host rhinoceros density (rhinoceros.ha ${ }^{-2}$ ).

\begin{tabular}{llllll}
\hline Model/Hypothesis & Source & $K$ & $\mathrm{AIC}_{\mathrm{c}}$ & $\Delta \mathrm{AIC}_{\mathrm{c}}$ & $\omega$ \\
\hline Host density & $\mathbf{( 1 )}$ & $\mathbf{3}$ & $\mathbf{5 0 7 . 4 8 6}$ & $\mathbf{0 . 0 0}$ & $\mathbf{0 . 9 3 0}$ \\
Mean max summer temperature *** & $\mathbf{( 2 )}$ & $\mathbf{3}$ & $\mathbf{5 1 4 . 1 2 3}$ & $\mathbf{6 . 6 4}$ & $\mathbf{0 . 0 3 4}$ \\
Max temperature * & $(3)$ & 3 & 515.084 & 7.60 & 0.021 \\
Min temperature * & $(3)$ & 3 & 517.383 & 9.90 & 0.007 \\
Base model & & 2 & 517.427 & 9.94 & 0.006 \\
Humidity * & $(3)$ & 3 & 519.885 & 12.40 & 0.002 \\
Fire regime & $(4)$ & 3 & 522.491 & 15.01 & 0.001 \\
Rainfall & $(3)$ & 3 & 523.000 & 15.51 & 0.000 \\
Population Age & & 3 & 526.305 & 18.82 & 0.000 \\
Founder population Size & $(5)$ & 3 & 527.218 & 19.73 & 0.000 \\
Summer rainfall ** & $(2)$ & 3 & 528.601 & 21.12 & 0.000 \\
\hline
\end{tabular}

Models are in descending order from most to least supported based on Akaike second-order Information Criteria $\left(\mathrm{AIC}_{\mathrm{c}}\right)$. The base model, highlighted in grey, included only the randomeffect for population. The confidence set of models, i.e. $>95 \%$ of Akaike weights $(\omega)$, are in bold. $\mathrm{K}$ is the number of parameters in a model. Number in parentheses refers to reference. *Mean value 2000-2011** During season of collection. The set of candidate models differs between parasite groups as they use different transmission methods. (1) Arneberg et al. 1998; (2) Haukisalmi and Henttonen 1990; (3) Atkinson et al. 2013; (4) Fuentes et al. 2007; (5) Paterson et al. 1998.

The directly transmitted strongyle-type nematodes were found in all populations except one, at a mean population abundance of 1918 eggs per gram (epg) (range: 44 - 4075 epg, SD = 1438). The indirectly transmitted cestode, Anoplocephala sp., was found in all populations at a mean population abundance of 228 epg (range: 33 - 546 epg, SD = 149). 


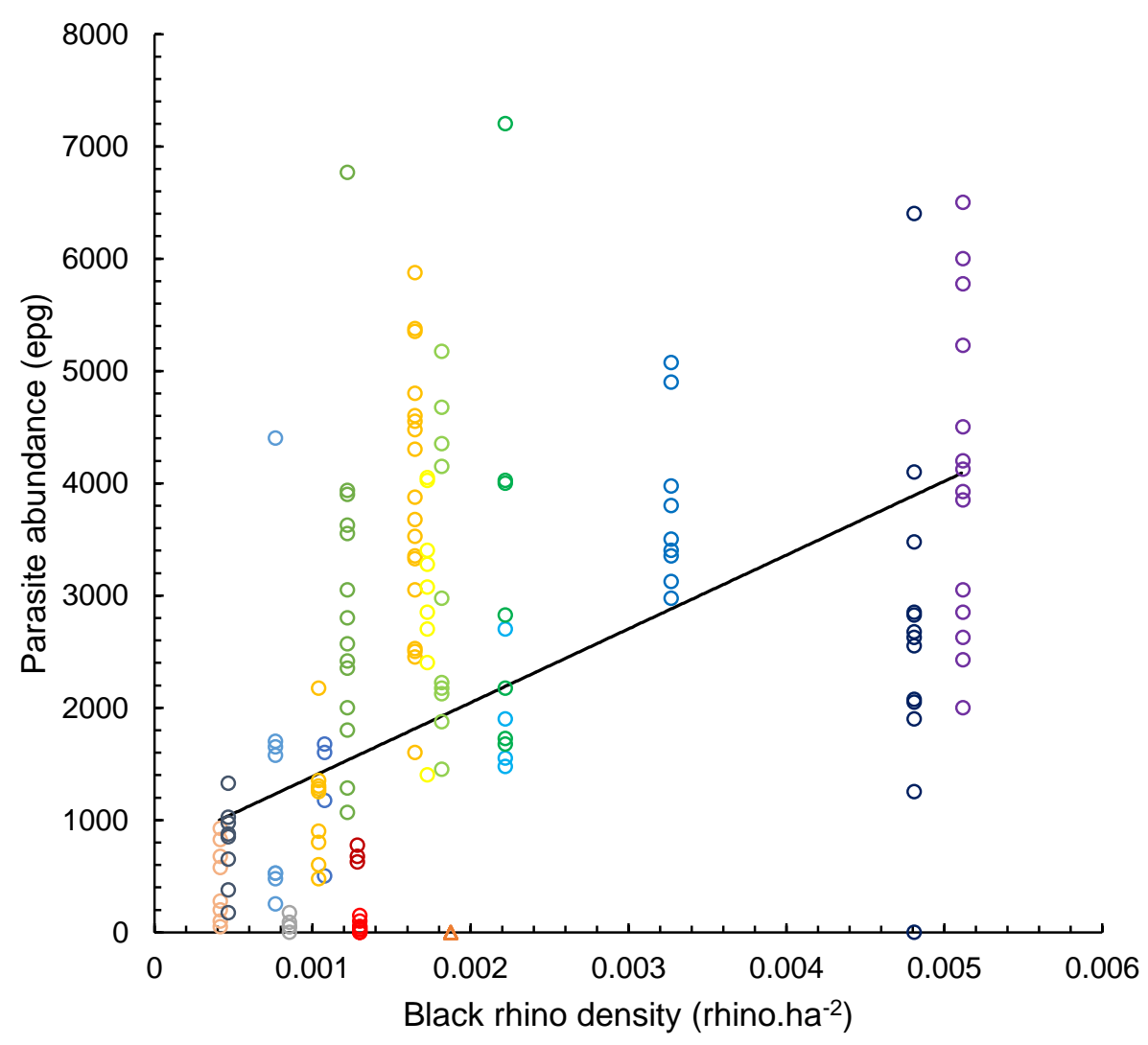

Figure 3.1 Scatter plot showing strongyle abundance in host populations against the density of black rhinoceros (rhinoceros.ha ${ }^{-2}$ ) in that population. Open circles represent individual samples $(n=160)$, and the colour of the circle can be used to differentiate between different populations. The triangle represents all samples that came from the single population that was sourced from a Namibian source population. The trendline represents the fitted line using parameters from our results (LMM).

Model fit when analysing the data utilising a GLMM with a negative binomial distribution and a log-link for the strongyle parasite group was poor. Further investigation revealed that the data did not follow a negative binomial distribution; skewness was low (0.5), with the mean egg count (2280 epg) close to the median (2150 epg). Also, kurtosis was very low (-0.5) indicating a wide flat peak to the data, and this was likely to be the reason why the data were considered non-normal based on the Kolmogorov-Smirnov test (statistic $=0.98, \mathrm{p}=$ 0.001). This distribution is likely a result of combining data from multiple populations. While each individual population has an aggregated parasite distribution with a single peak, when combined, the different peaks for each population will create multiple peaks. This ultimately 
results in a single wide flat peak, which when viewed in a p-p plot does not seem to differ meaningfully from normality. This does not occur for Anoplocephala sp., as egg counts for the different populations were much less variable. The competing set of models taken from single factor model tables did not differ between using a GLMM, and a LMM on untransformed data for the strongyle group. Hence, results from the LMM are presented and plotted (Figure 3.1). While using a GLMM, no two factor model improved on the top model. However, when using a LMM all two-factor models containing the top model improved on the original model, while all three factor models including the top two models improved on all two factors models. This is a clear sign of over-parameterization, hence only one factor models for the strongyle group are presented.

Using multi-model selection and inference to explain parasite abundance, host density models performed best for the directly transmitted, strongyle parasite group (Table 3.2, Figure 3.1, coefficient 657404.5, std. error 212871, intercept 730.5, std error 485.9). Host density contributed $100 \%$ of Akaike weights $(\omega)$, while all other models were implausible, receiving no support. For the indirectly transmitted Anoplocephala sp., the host density model performed best, contributing 93\% of Akaike weights (Table 3.3, Figure 3.2, coefficient 235.4, std error 117.0, intercept 4.8, std error 0.3). Parasite abundance was again not explained by most environmental parameters, although mean max summer temperature was included in the confidence set of models $\left(\sum \omega>0.95\right)$ (Zuur et al. 2009).

Each predictor was added to host density to see if models with two fixed effects could improve on the host density model for Anoplocephala sp. A model containing host density and mean max summer temperature was the only model to improve on the original host density model $\left(\Delta \mathrm{AIC}_{\mathrm{c}}=-0.35\right.$, max temp coefficient -0.06 , std. error 0.09 , host density coefficient 188.1, std. error 142.4, intercept 7.1, std error 3.0). However, auto-correlation between the two fixed effects in the model means that estimates of these parameters are not reliable, and the model table comparing the single fixed effects (Table 3.3) should be utilised. 


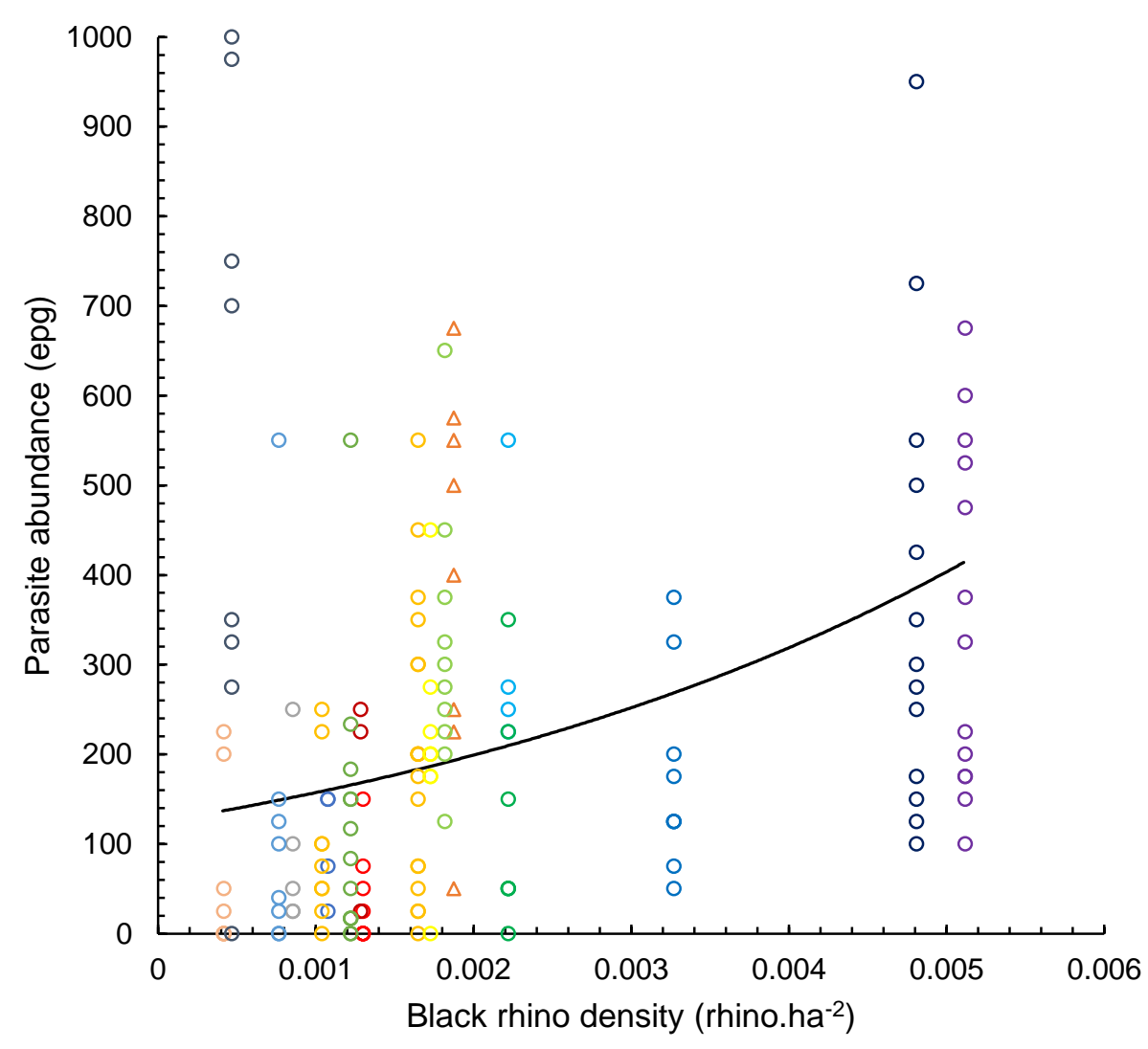

Figure 3.2 Scatter plot showing Anoplocephala gigantea abundance in host populations against the density of black rhinoceros (rhinoceros.ha ${ }^{-2}$ ) in that population. Open circles represent individual samples $(n=160)$, and the colour of the circle can be used to differentiate between different populations. Triangles represent individual samples that came from the single population that was sourced from a Namibian source population. Two data points are not included in this graphic for ease of display (2516 epg x 0.0012 rhinoceros.ha $^{-2}$, 1875 epg x 0.0051 rhinoceros.ha ${ }^{-2}$ ). The trendline represents the fitted line using parameters from our results (GLMM).

\subsection{Discussion}

For directly transmitted parasites, we conclude that host density is the only predictor in this study that influences population-level parasite abundance. This is despite numerous other hypotheses predicting that environmental factors, such as temperature, rainfall, and humidity, will influence larval survival and hence are of importance to parasite abundance (Haukisalmi and Henttonen, 1990; Paterson et al., 1998; Mouritsen and Poulin, 2002; Fuentes et al., 2007; Atkinson et al., 2013). The relationship between primary host density and parasite abundance 
for indirectly transmitted parasites is thought to be more complex due to the influence of secondary host density (Stien et al., 2010). Despite this we show a clear relationship between primary host density and parasite abundance for the indirectly transmitted Anoplocephala sp. (Figure 3.2). Secondary host density does not mask the relationship between primary host density and parasite abundance for Anoplocephala sp. and its black rhinoceros host, perhaps because of the ubiquity of the secondary host across the landscape and between different populations of black rhinoceros (Van Nieuwenhuizen et al., 1994).

The host density model was marginally improved for Anoplocephala sp. by also including mean max summer temperature. This weak negative relationship between summer max temperature and parasite abundance has been found previously in other mammal-cestode relationships, and is likely due to egg desiccation reducing abundance in hotter areas (Haukisalmi and Henttonen, 1990).

Strongyle nematode eggs were found in all populations, except the single population that originated from a Namibian source population. The Namibian population's habitat and rainfall is very different from all directly studied populations, and it also has an extraordinarily low black rhinoceros density (i.e. 0.00013 rhinoceros.ha ${ }^{-2}$ ). Hence, it is possible that strongyles became extinct or never existed in the source population, or were not translocated with the host when reintroduced to the South African reserve.

There may be other mechanisms, rather than density-dependent transmission, that cause host density to drive parasite abundance. An individual's parasite abundance has been linked to their age. If a population is founded with younger individuals, then, as that population ages and grows denser, the age-structure within the population may change. However, in this study the age of a population had no impact on parasite abundance.

There was little or no support for models including only the random effect compared to mixed-effect models - indicating that the fixed-effects in the supported models had substantial value in explaining variation in the response variable (parasite burden). The leading mixedeffect models might still be improved in the future by the addition of other fixed-effects not tested here. These mechanisms may include host susceptibility, where inbred hosts may be more susceptible to parasite invasion, and within-host parasite community interactions, where competition and facilitation between parasite species may limit or increase parasite abundance (Pedersen and Fenton, 2007). For instance, it has been shown that host susceptibility may affect variation in parasite abundance between individuals (Paterson et al., 1998; Poulin, 2007b). 
Black rhinoceros are a critically endangered and elusive species which meant that samples were not readily available, and sample size for some populations was very low. However, black rhinoceros may be an excellent model organism for testing these hypotheses, due to their solitary nature (Altizer et al., 2003), the slow rate of any natural changes in population size, and the host species specificity of the parasites studied. We also used modern statistical techniques that meant populations did not have to be reduced to a single statistical average (mixed-models). Furthermore, by utilising the fortuitous experiment created by the translocation and reintroduction of black rhinoceros in South Africa we were able to compare multiple populations of the same host-parasite interaction, a novel method for investigating epidemiological hypotheses. Hence, despite the restrictions of small sample size, and using faecal egg counts rather than directly measuring abundance, we were still able to show that host density is a singularly powerful predictor of macroparasite abundance for a directly transmitted parasite, and a highly influential predictor within an indirectly transmitted parasite.

Our demonstration of a previously assumed mechanism for parasite abundance gives real-world evidential support to epidemiological theory. This may have a wide range of implications for parasite control (Gortázar et al., 2006), disease prevention (Anderson and May, 1991), and conservation (Woodroffe, 1999). The assumption of density-dependent transmission is often used for determining the basic reproductive number (Anderson and May, 1991) - the expected number of secondary cases arising from one infected individual, and hence underpins much of current epidemiology. It also underpins some key questions in parasitology, such as whether parasites with low levels of virulence can regulate host population size (Hudson et al., 1992). The dominance of host density in explaining population-level parasite abundance also supports the expectation of a population density threshold (Lloyd-Smith et al., 2005), below which a parasite will become extinct. This chapter, then, gives support to many current parasite and disease control programs that work to reduce the density of susceptible individuals. As susceptible host density is a determinant of parasite abundance, reducing susceptible host density by rising vaccination rates should increase the likelihood of local parasite extirpation. 


\subsection{Post-publication addendum about sampling and analysis robustness}

An important concern for the analyses in this chapter was that a number of populations did not have the optimal number of samples for a precise estimate of parasite abundance as identified by Chapter 2. Hence, an analysis of the robustness of the data is presented here. Also included in this analysis is an assessment of pseudo-replication, as individuals relating to each faecal pile could not be identified.

All other factors being equal, reduction in sample size will result in reduced power to detect trends. Hence if reductions in sample size, by removing less sampled populations or samples within populations that are more likely to be from the same rhinoceros (i.e., spatial auto-correlation and pseudo-replication), generate the same patterns, then we can eliminate within-population sampling bias as an influence on the trends and patterns observed. Below, I do both of these analyses for the robustness of the outcomes.

\section{Testing for the influence of spatial auto-correlation and pseudo-replication}

As defecation had not been directly observed, there was the possibility that two samples may have come from the same individual (pseudo-replication), especially if those samples are nearer one another. Sources of spatial auto-correlation, other than repeatedly but unknowingly sampling the same individuals are also possible and need to be allowed for should they exist. Where these are the case, repeated sampling is less likely to represent variation in parasite abundance within and across the population. Variance within populations could be underestimated and translate into erroneous relationships across populations.

A variety of field signs could be used to predict whether faecal samples were from different individuals. For example, if an individual was walking with a calf (identified either from footprints or dung) it was likely to be a female. Also, the behaviour of rhinoceros during defecation events and at middens, such as kicking their dung pile and scraping their feet through it, is sex-specific (Freeman et al. 2014) such that these cues can be used to identify sex. Adult males scrape and kick faeces for significantly longer distances than females (adult male mean scrape length $323.32 \mathrm{~cm}$, SE 40.94, adult female mean scrape length $154.63 \mathrm{~cm}$, SE 40.21, Freeman et al. 2014), hence scrapes $>300 \mathrm{~cm}$ in length were identified as male. If boluses were at adjacent middens and field conditions allowed, footprints could also be tracked backwards and forwards. Alongside the estimated defecation time of the bolus, this allowed me to predict 
whether dung piles were from the same individual. Where field signs could not be used to differentiate between individuals, I ensured a $>1 \mathrm{~km}$ distance between faecal samples. Local knowledge of home ranges was also used to increase the likelihood that samples came from different individuals. On a population level, I prioritised sampling dung piles at greater distances from each other such that samples were distributed throughout reserves. Nevertheless, some spatial auto-correlation and multiple sampling of the same individual rhinoceros (pseudo-replication) may still have occurred. This understanding motivated the following check for spatial-autocorrelation, including pseudo-replication within my faecal parasite count dataset.

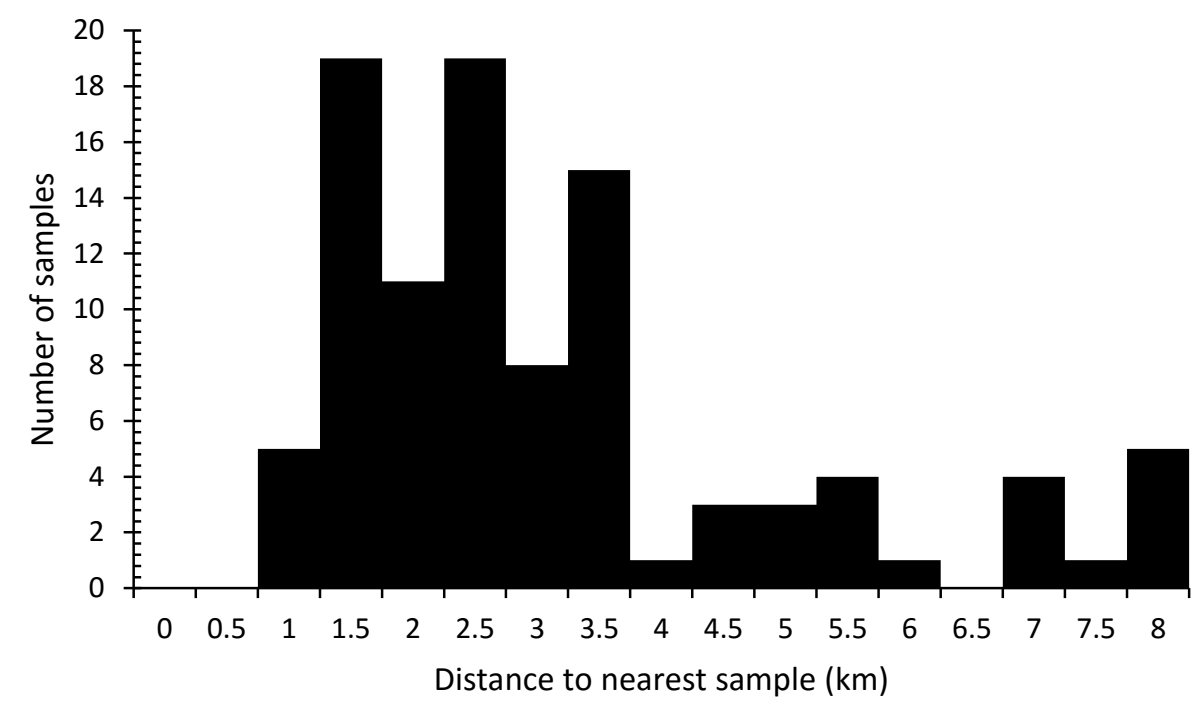

Figure 3.3 A histogram of the distance to the closest sample from each sample

Using all samples across all populations that were not differentiated using field signs, the mean distance to the closest sampled dung pile was $2.7 \mathrm{~km}$ (Figure 3.3). Here I increase the minimum distance between samples, by removing two samples from each population (except a single population where all samples were thought to be from different individuals based on local knowledge of home ranges). Each removed sample was one half of the two pairs of samples that were spatially closest. This was chosen over increasing the minimum distance between samples (currently $1 \mathrm{~km}$ ) as such a method would bias the robustness analysis; removing more samples from smaller, more dense populations. 

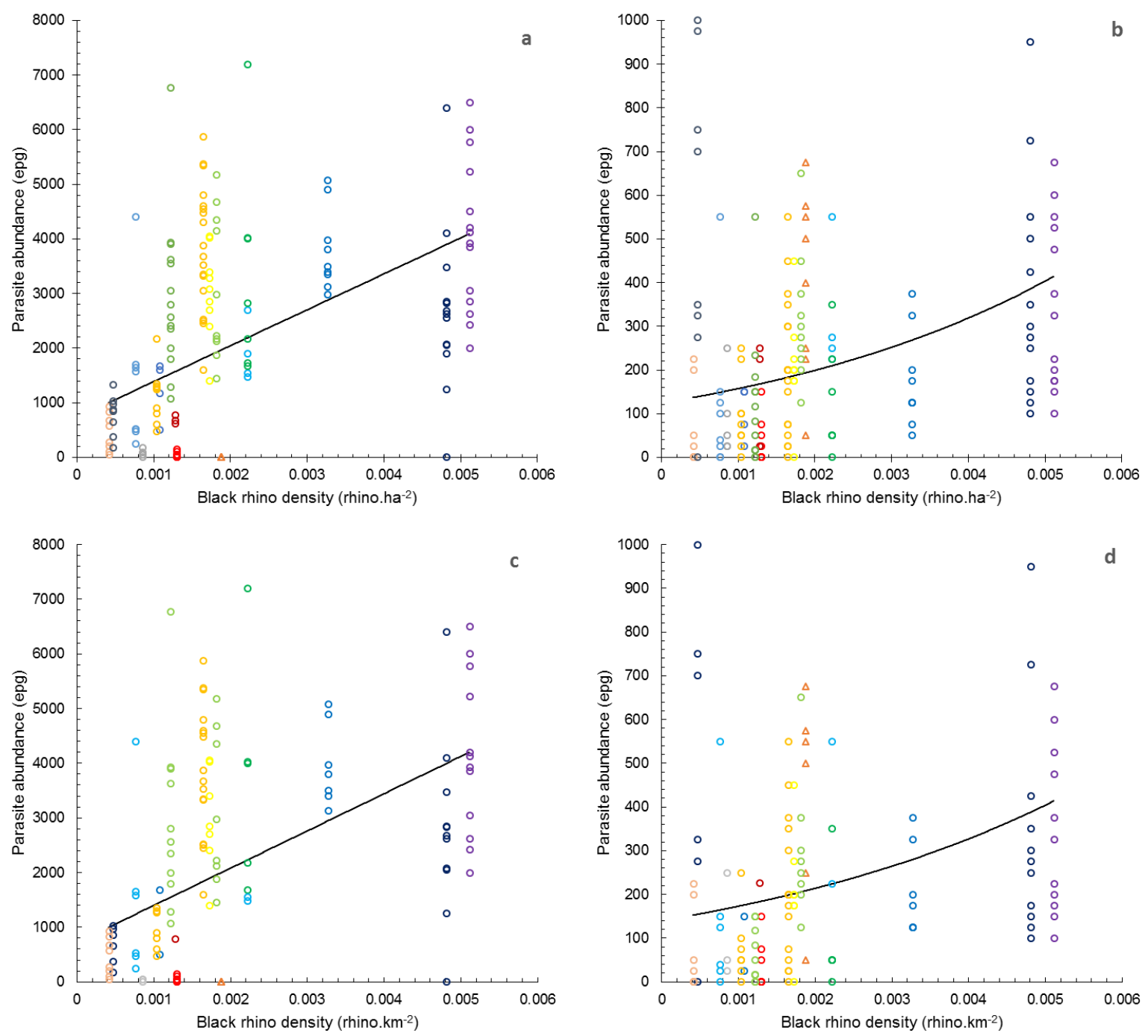

Figure 3.4 How samples in close proximity to each other affect the fitted model between host density and parasite abundance (a) strongyles with all samples $(\mathrm{n}=160)(\mathrm{b})$ Anoplocephala sp. with all samples $(n=160)(c)$ strongyles with reduced sample size $(n=126)(d)$ Anoplocephala sp. with reduced sample size $(n=126)$

This test for robustness removed 34 samples from the dataset of 160 . Fifty-seven samples with other evidence, such as field signs that indicated samples geographically close came from different individuals, were still included. The mean distance to the closest sampled dung pile for all populations increased from $2.7 \mathrm{~km}$ to $4.1 \mathrm{~km}$. Plotz (2014) measured a 1.54 $\mathrm{km}$ average daily displacement (movement) amongst black rhinoceros in Hluhluwe-iMfolozi Park, a key park within this study. Moreover, the same rhinoceros had on average a $9.77 \mathrm{~km}^{2}$ (average 95\% minimum convex polygon) home range, which would equate to a diameter of 
$3.5 \mathrm{~km}$ were they approximated by circles of equivalent area. Hence, this increased distance between samples should increase the chance that samples represent different individual rhino.

By comparing the analysis from the full dataset with this smaller dataset that excluded near-samples, I test whether spatial auto-correlation and resampling of the same individuals (pseudo-replication) explains the trends in parasite abundance across reserves. All other factors being equal, we would expect a reduction in sample size to reduce our likelihood of detecting the same relationship. Thus, where the relationship persists we can conclude that sample size and distribution do not play a role in the relationship and the relationship is not an artefact of spatial auto-correlation. In the re-analysis, the direction of the key relationships of Chapter 3 amongst strongyles, Anoplocephala sp. and host density are still evident (Figure 3.4). The continued strength of the relationships, despite a much reduced sample size, gives confidence that the influence of spatial auto-correlation including resampling the same individuals, is not a substantial influence on my results. These analyses of dataset and relationship robustness provide further confidence that spatial auto-correlation, especially pseudo-replication, are not a determining factor of analysis outcomes.

\section{Testing for sample size influence}

In Chapter 2, I concluded that ten or more samples are needed to accurately determine the mean parasite abundance within a population. Nevertheless due to sampling constraints, five of my 18 populations yielded fewer than eight samples. Fortunately, the objective of Chapter 3 is a population-level analysis. Thus, rather than discard useful information (data), I use a random effect for site in my analysis. The random-effect makes the analysis of parasite abundance across populations and inclusion of sites with different, sometimes small, numbers of samples possible by treating each sample as a repeated measure within each site. This means that varying numbers of samples within sites has much less of an influence on my final results than the numbers of sites sampled. However, ideally I would have been able to collect at least ten samples from each site to capture the variation in parasite abundance from a population. Hence, small sample size is still a concern. In an attempt to address this concern, I here reject populations with small sample size and replicate the analysis to see how they may be affecting the model fit. 

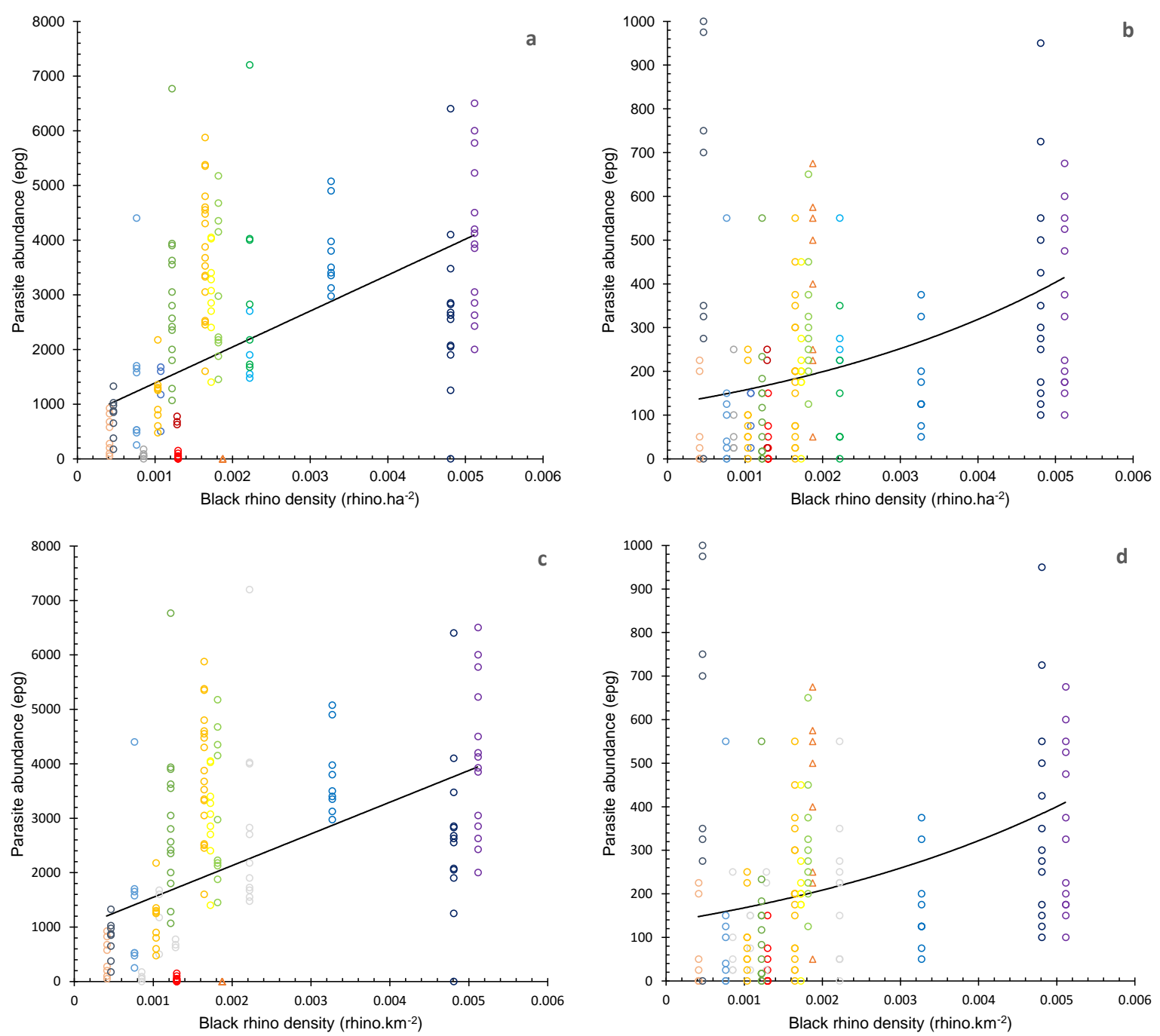

Figure 3.5 How removing populations with low sample size affects the fitted model between host density and parasite abundance. Data points in light grey have been removed from the analysis. Trend lines represent the new model fit (from Chapter 3) after data points have been removed from the analysis. (a) Strongyles with all samples, (b) Anoplocephala sp. with all samples, (c) Strongyles from populations with $<8$ samples removed, (d) Anoplocephala sp. from populations with $<8$ samples removed.

The relationship between the precision and accuracy of a parasite abundance estimate and sample size is not linear. Thus, having one or two samples fewer than the desired sample size $(\mathrm{n}=10)$ has minimal impact, but as sample size declines further the impact on estimate accuracy and precision increases (Figure 2.3). Thus, of greatest interest is whether excluding sites where sample size was less than eight changes the outcomes of my analysis and conclusions. In Figure 3.5 below I show my best model from Chapter 3 (host density), but have 
rejected all populations with $<8$ samples, leaving 13 populations. The relationships observed were robust to the loss of five sites with fewer than eight parasite counts each, and my key result is still supported. In conclusion, the sample size for some sites was far from ideal or optimal, but my sample size should still give a reasonable indication of parasite abundance across populations, and the main conclusion of my results is still supported even when five populations $(28 \%)$ have been removed. 
Chapter 4: Investigating the effects of parasitism on the body condition and sexual development of black rhinoceros (Diceros bicornis)

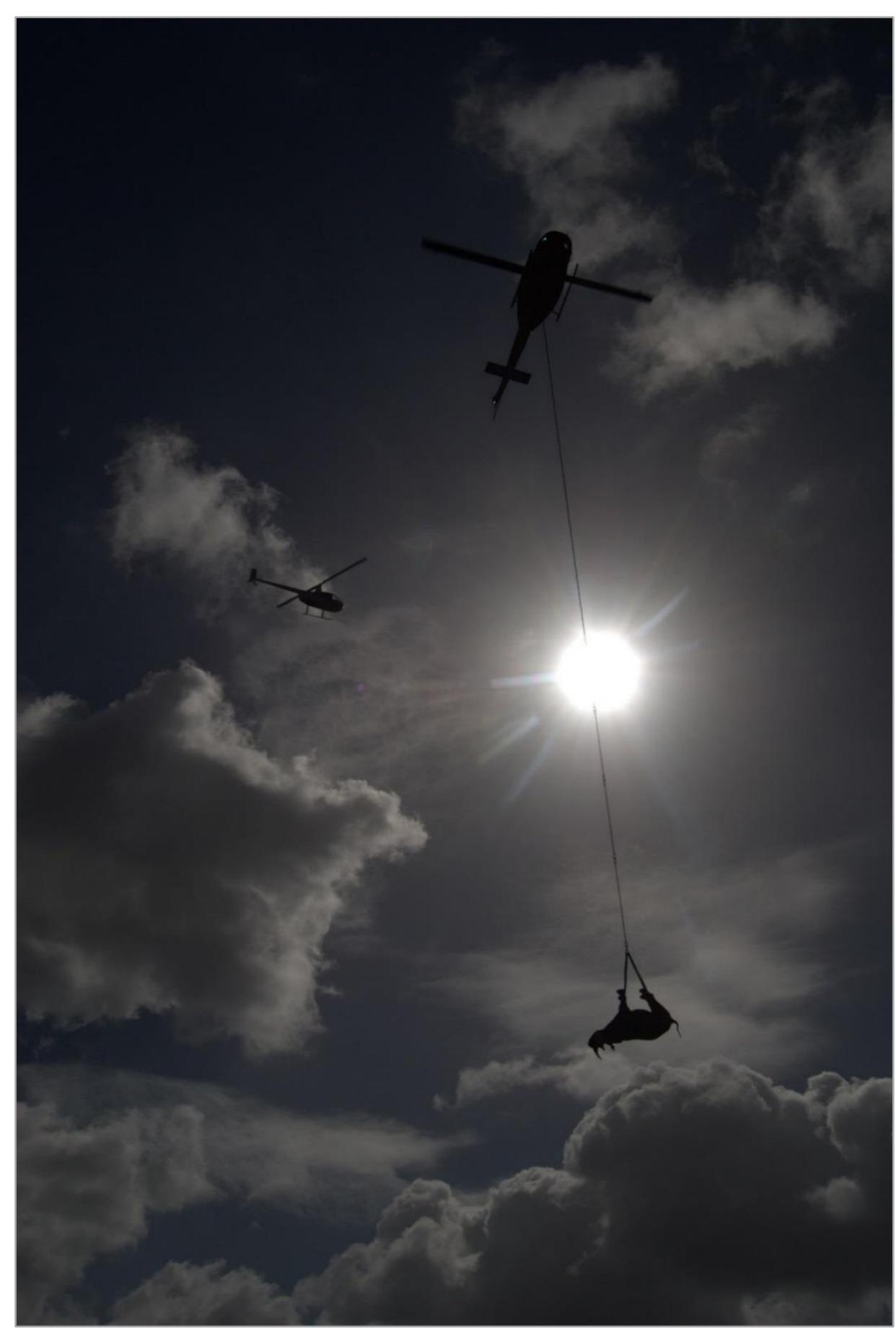

Black Rhinoceros range expansion project (WWF), Eastern Cape, Oct 2012, C Andrew Stringer.

"The rhinoceros is a very heavy sleeper and his loud snoring betrays his lair." Joseph Delmont (1931) 


\subsection{Preface}

While the previous chapter investigated the determinants of population level parasite abundance, the following chapter focuses on some determinants of parasite intensity on an individual level, such as age \& sex. This is potentially useful for conservation purposes as it will allow individual rhinoceros that are at greatest risk of high parasite burden to be identified. This had not been previously attempted in black rhinoceros due to logistic difficulties. Here it was made possible as I was provided with the rare opportunity to study black rhinoceros captured for translocation. Focusing on the individual, I also attempt to measure the impacts of parasitism on novel measures of host resources, such as the size of sexually selected characteristics. These may be more obviously affected by smaller parasitic effects, and hence provide a 'canary-warning' of more widespread parasitic effects. 


\subsection{Abstract}

Parasites have been shown to decrease fecundity and increase the mortality rates of hosts. This is thought to be because they take resources from their hosts. The link between parasite abundance and resource budgets in black rhinoceros (Diceros bicornis) has not been established - probably because opportunities to study this are limited in this critically endangered megaherbivore. Here I investigated the development of sexually selected traits and the influence of parasite burden in young black rhinoceros. In particular, I attempted to link parasite abundance to the size of sexually selected characteristics, thought to be highly vulnerable to parasite resource demand, and a number of measures of body condition. I evaluated parasite abundance on 39 black rhinoceros captured for translocation and reintroduction from a population with very high parasite abundance. Three intestinal helminths, the strongyle nematodes, Anoplocephala sp and Oxyuris sp, as well as the abundance of ticks, were assessed. Here I found that younger individuals harbored very high levels of parasitism, but found no differences in parasite abundance between sexes. I found four sexual dimorphisms, with males having a faster-growing anterior horn length, circumference, volume, and body length. Parasite abundance within an individual had no impact on the size of sexual dimorphisms or other measures of body condition. These results help identify potential avenues for future research, for instance, the cause of the large variation in the growth rate of anterior horn size could be investigated. 


\subsection{Introduction}

Parasites take resources from their hosts. Parasites with high levels of virulence may have clear impacts on mortality (see 1.3.1). Parasites with low levels of virulence have been shown to affect host growth rates (Forbes et al., 2000; Collyer and Stockwell, 2004), fecundity (Forbes, 1993; Albon et al., 2002; Irvine, 2006) and longevity (Gulland, 1992; Murray et al., 1997; Collyer and Stockwell, 2004), and do so by reducing an individual's resource budget (Gunn and Irvine, 2003, see 1.3.2). Many parasites do not directly cause mortality, allowing them to persist within individuals for a long period of time as well as infecting a large proportion of a population. Quantifying their level of virulence, then, is important for conservationists as it allows their impacts on population performance to be assessed.

Black rhinoceros are a critically endangered megaherbivore. Maximising population performance is seen as key to long-term conservation efforts (see 1.5.2). Previously, trypanosomes and babesia parasites have been studied within black rhinoceros as they were suspected causes of mortality (Mihok et al., 1995; Nijhof et al., 2003). To my knowledge, the impacts of parasites with low levels of virulence on black rhinoceros have never been attempted. This may be because measuring the impacts of parasites in a megaherbivore is difficult. For instance, physically capturing individuals to measure condition is often logistically challenging.

A variety of parasites infect black rhinoceros. It is possible to estimate their impacts on black rhinoceros by looking at similar host-parasite relationships (see 1.6.2). For instance in horses, strongyle nematodes are known to be associated with low protein levels in the blood (hypoproteinemia), and the pinworm Oxyuris sp rarely causes detectable symptoms. Tapeworms absorb nutrients from the gut rather than feeding from the intestinal wall. However, tapeworms from the family Anoplocephalidae have been shown to have a wide variety of differing levels of virulence (Narsapur 1988). Finally, ticks take resources from their hosts directly through blood ingestion. The impacts of ticks can be considerable, for instance, weight gain was reduced by $44 \mathrm{~kg}$ per year in cattle calves raised with a moderate tick burden (Norval et al., 1988). However, all these examples relate to domesticated rather than wild individuals. The situation with wild individuals may be quite different due to further nutritional pressures, not usually experienced in captivity, exacerbating the impacts of parasitism.

Macroparasites are aggregated within a small proportion of the host population and it is within these individuals that you would expect the effects of parasitism to be greatest. 
Numerous factors such as age and sex may make an individual more likely to have a high abundance of parasites. For instance, exposure to parasitism increases with age, while acquired immunity develops with age. However, there are further influencing factors on the immune system, for instance, immune function declines in old age. Also, the acquired immune response to a specific pathogen is exposure dependent and the age at exposure may be highly variable; changing between different individuals and populations. These mechanisms may combine to create an age range where peak parasite intensity is most likely (see 1.2.1). Sex may also impact parasite intensity, potentially due to the differing impacts of different sex hormones on the immune system (Schalk et al., 1997). Furthermore, parasites may be competitive or facultative, hence, the intensity of one parasite within a host may affect the intensity of another (see 1.2 and 1.3). All these factors influence which individuals are most likely to experience the greatest impacts from parasitism.

Determining the level of virulence of a parasite has generally proven difficult. For instance, even when parasite-induced detrimental effects are known to occur, indices of an individual's resource budget have not correlated with parasite load (Stien et al., 2002; SchulteHostedde and Elsasser, 2011). This may be because there are many potential influences on an individual's resource budget, such as stress, habitat quality of the home range, and inter- and intra-specific competition. Sexually selected traits may be more likely than other phenotypes to reflect the impact of parasites on host resources. Parasite-mediated sexual selection is the theory that ornaments advertise an individual's ability to resist or overcome the impacts of parasites (Hamilton and Zuk, 1982; Able, 1996). Hence, measuring sexually selected characteristics may give a reliable indication of the impact of parasites on lifetime resource budgets (Ezenwa and Jolles, 2008). The black rhinoceros has a 1:1 adult sex ratio, is body-size sexually dimorphic (males are 11\% larger, on average, than adult females, Owen-Smith 1988), and a larger female than male investment in offspring. Using first principles, this indicates that sexual competition between males is likely to be intense, facilitating the expression of sexually selected characteristics (Clutton-Brock and Vincent, 1991).

Energy taken into the body is thought to be allocated to either reproduction, growth, maintenance or storage (Perrin \& Sibly 1993). Here I use the term resource budget to identify where energy has been allocated to either growth or storage. Overall growth can be estimate by measures of body size, while stored energy can be estimated using measures of body condition. Commonly used measures of body condition attempt to identify the levels of excess resources within individuals. These attempt to measure the size of energy reserves within an 
individual such as fat and protein, relative to the rest of the population (Peig and Green, 2009; Labocha et al., 2014). Measures of body condition may be visual, such as by assessing body fatness from external cues such as the visibility of bony protrusions (Reuter and Adcock, 1998). Other measurements use combinations of body measurements, such as length and weight, to estimate whether individuals are larger in girth, or heavier, than would be predicted by their body length. Ratios of two body measurements may be used, for instance the ratio of body weight to body length. However, ratios cannot be used where there is variation in body size (e.g. juveniles and adults), as ratios are influenced by body size (Labocha et al., 2014). Alternatively, residuals may be used, for instance from the regression of body weight to body length (Schulte-Hostedde et al., 2005; Schamber et al., 2009).

In this chapter I first investigate the distribution of parasites through a sub-population of black rhinoceros captured for translocation, examining whether age and sex of the host, or the intensity of other parasites within the host, affects parasite abundance. I then attempt to identify key sexual dimorphisms within black rhinoceros, which may represent sexually selected traits. Finally I utilise the size of sexual dimorphisms, alongside a number of other measures of body condition, to investigate whether the resource budget of black rhinoceros is reduced in highly parasitized individuals. I investigate the levels of virulence for four parasite groups - strongyles (Strongylida), pinworms (Oxyuris sp.), tapeworms (Anoplocephala sp) and ticks (Ixodida).

This chapter focuses on the development of sexually selected traits and the influence of parasite burden in young black rhinoceros. I focus on younger black rhinoceros that are predominantly still in a period of development. The focus on this age range helps to test whether highly parasitized rhinoceros experience a developmental delay in the growth of sexually-selected characteristics. Also, developing animals may be more vulnerable to the impacts of parasitism due to the stressful events in this period, such as the separation of mother and calf, and the establishment of a home range. Black rhinoceros are no longer dependent on their mothers at age 4, but may be viewed as developmentally mature by 7 years old in females and somewhat older in males (see 1.5.1).

\subsection{Materials and Methods}

Thirty-nine black rhinoceros immobilized for translocation and reintroduction were investigated for their parasite abundance. The process of rhinoceros capture is an extraordinary and stressful event. This may have a number of potential effects on the parasites of captured 
hosts. Potential influences are further explored in Appendix 1.

All individuals came from the same reserve in the Eastern Cape of South Africa at two translocation events (Sep-Oct 2011 and Feb-Mar 2012). The reserve contained a healthy and still expanding population of black rhinoceros (Law et al., 2013). Captured individuals were of known age ( $\pm 1-2$ months) and were predominantly younger animals, with 36 individuals less than 9 years old, and three fortuitous extra samples from older individuals (Range $=30-207$, $\bar{x}=71.7$ months). Rectal faecal samples $(n=30$; due to a lack of faeces within the rectum at the time of capture faecal samples could not be obtained from nine individuals) were taken to investigate two gastrointestinal parasite groups - strongyle nematodes and Anoplocephala sp. A modified McMaster technique was used to count the helminth eggs in faecal samples as previously described. Two tick species were found on captured black rhinoceros, Amblyomma hebraeum and Rhipicephalus zumpti. The total number of ticks directly around the anus was counted and used as an indication of total tick burden $(\mathrm{n}=38)$. Finally, the Scotch Tape Test was used to identify the presence or absence of Oxyuris sp. eggs on the anus ( $n=34$; in some circumstances the test could not be used as water had been poured over the anus before the test could be performed) (Zajac and Conboy, 2006). However, this test only reveals the presence or absence of Oxyuris eggs. Hence, further analysis with Oxyuris sp. compared infected vs uninfected individuals rather than abundance.

A variety of body measurements were recorded in an attempt to identify sexual dimorphisms in black rhinoceros. These included the length and base circumference of both anterior and posterior horns, half girth (measured from the midline of the most dorsal point at the level of the shoulder, through the axilla, and continuing this line to the midline of the sternum), shoulder height (taken from the base of the foot, while the front limb was elevated to horizontal, to the midline of the most dorsal point at the level of the shoulder), and body length (measured by using a tape measure placed on the midline between the base of the ears, held along the midline to the most dorsal point at the level of the shoulder, then held to the midline at the rump, and finally held straight to an estimation of 90 degrees to the base of the tail). Measurements were rounded to the nearest $10 \mathrm{~mm}$. An approximation of the volume of the anterior horn was also estimated based on the volume of a cone, and utilizing the measured length and base circumference of the horn. A total of 19 male and 20 female rhinoceros were measured.

To test for sexual dimorphisms, body and horn measurements were plotted against the 
age of individual rhinoceros. Measured characteristics all came to an asymptote with increasing age, hence age was log transformed to normalise the variable before a linear regression line was fitted for each sex. The difference between the regressions for each sex was then tested using a multiple regression. A significant value for the sex model indicated differences in the intercepts of the two regression lines, while a significant value for the interaction between sex and the body measurement indicated differences in the slopes of the two regression lines. I then used the residuals from the regression lines to show oversized or undersized sexual dimorphisms amongst parasite-sampled individuals. These residuals were checked against age to ensure that any effects of age had been removed.

I also use three measures of body condition. The first uses body measures to see whether an individual had a larger or smaller girth than may be predicted by their body length $(\mathrm{n}=28$; body measurements could be obtained for 37 rhinoceros, a rectal faecal sample could not be collected from nine of these). Due to a variety of ages in the study population, and hence body sizes, I utilise the residuals from a linear regression of half girth to body length. The second utilises body length and the body weight of an individual, to see whether an individual had a higher or lower body weight than may be predicted by their body length ( $\mathrm{n}=17$; due to logistical difficulties only 19 rhinoceros could be weighed, a rectal faecal sample could not be collected from two of these). Again, residuals from a linear regression of body weight to body length were utilised. Total body weight was measured when an individual was in a crate for transportation, and the weight of the crate was then subtracted. These methods assume an isometric relationship between body size and body weight. This assumption may be flawed as it has been shown that larger individuals may have proportionally more resources for their size. A scaled mass index that incorporates potential non-isometric scaling differences can be used to overcome this problem (Peig and Green, 2009). However, the relationship between body weight and body length for my sample was linear, perhaps because of the focus on younger individuals, and hence a scaled mass index was not used in this case.

The third method of estimating body condition was a visual assessment. Representative photos of key areas of body were taken when the individual was lying down. The body condition of specific body areas was assessed on a scale of 1 to 5 using methods described in Reuter and Adcock (1998). An online survey of photographs was completed by nine individuals, all who had at least undergraduate zoology training, in a double-blind format. 


\section{Statistical methods}

To describe parasite aggregation, the corrected moment estimation of $\mathrm{k}$ was used, as described in Chapter 2. I tested the influence of parasite abundance on the size of sexual dimorphisms and body condition measures using two approaches. The first utilised a linear regression to test the influence of a parasite group on the sexual dimorphism or measure of condition (although a generalised linear model was used for Oxyuris sp. as the data were binary).

Macroparasites are not evenly distributed through host populations (see 1.2.1). A common rule is that $20 \%$ of hosts contain $80 \%$ of parasite abundance (Woolhouse et al., 1997). It is within these heavily parasitized individuals that the greatest effects would be expected. Hence, I also used a t-test to compare individuals with very high parasite intensity (top $20 \%$ of individuals) with all other individuals.

\subsection{Results}

\section{Parasite distribution}

The level of aggregation for all strongyles $(\mathrm{k}=3.2)$ and Anoplocephala sp. $(\mathrm{k}=1.0)$ were within the ranges seen in Chapter 2 for strongyles (k between 3.1 and 10.8) and Anoplocephala sp. (k between 0.1 and 4.6). There was no correlation between the abundance of different parasite types within individuals. Also, whether an individual had a very high parasite intensity had no influence on the abundance of other parasite groups within that individual (strongyles $\mathrm{t}_{28}=0.68, \mathrm{p}=0.5$, Anoplocephala sp. $\mathrm{t}_{28}=-0.87, \mathrm{p}=0.4$ ).

\section{Age and Sex}

Male and female black rhinoceros harboured similar levels of parasite abundance with no discernible difference in parasite intensity or prevalence between sexes. However, levels of aggregation may be different between sexes, as females potentially harbour a more aggregated distribution of parasites (strongyles $\mathrm{k}=2.7$, Anoplocephala sp. $\mathrm{k}=0.8$ ) than males (strongyles $\mathrm{k}=4.6$, Anoplocephala sp. $\mathrm{k}=1.9$ ). However, this may be due to a very highly parasitized individual female increasing the level of aggregation for both the strongyles (Figure 4.1) and Anoplocephala sp. (these were not the same individual). 
The age of captured individuals ranged from 30 to 205 months (2.5 - 17.1 years), although 36 of 39 captured rhinoceros were $<108$ months (9 years) of age. No correlations between age and parasite infection intensity or prevalence could be detected for any of the parasite groups. However for strongyles age may have influenced parasite distribution (Figure 4.1), as younger hosts were potentially more likely to have very high parasite loads. The mean age of those with the highest parasite loads was 47 months ( $\mathrm{SD}=10.7)$, in comparison to a mean age for the rest of the sampled individuals of 80.6 months $(\mathrm{SD}=43.3)\left(\mathrm{t}_{28}=1.9, \mathrm{p}=\right.$ 0.07). Due to sample size, the potential for type II error here is likely. A calculation of statistical power $(\beta=0.2, \alpha=0.05)$, shows that with the current mean ages and standard deviations, 60 samples would need to be sampled (currently $n=30$ ). However, our sample of age ranges was restricted by opportunistic sampling of individuals captured for translocation and if the mean age for individuals with a low parasite abundance had been 100 months, only 24 samples would be needed.

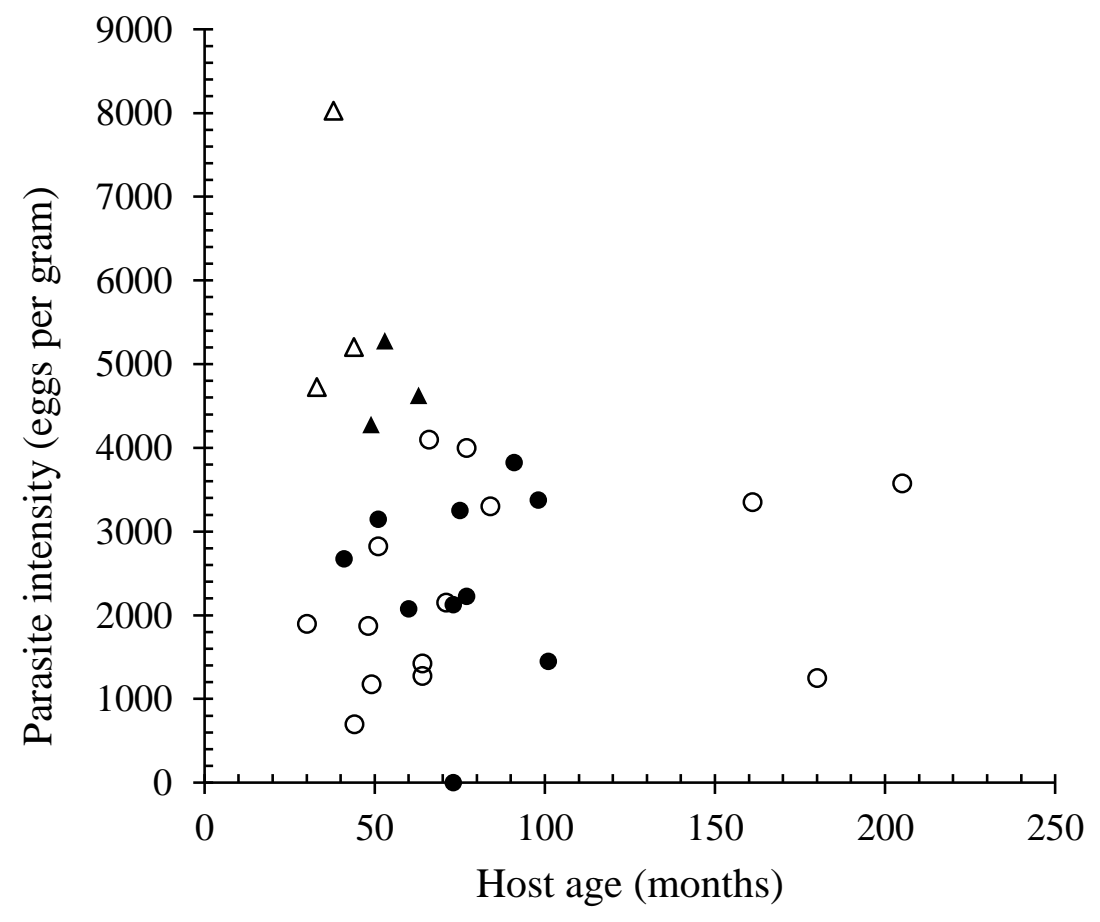

Figure 4.1 The estimated strongyle parasite intensity from rectal faecal samples of captured black rhinoceros of different ages. Filled symbols are males, open symbols are females, triangles highlight the top $20 \%$ of individuals with the highest parasite load, all other individuals are represented by circles. 


\section{Sexual dimorphisms}

Sexual dimorphisms were detected in anterior horn length, circumference, volume, and body length (Figure 4.2). All of these measurements differed significantly between sexes in the intercepts of regression lines, while only anterior horn length was the exception in not also differing significantly in the slope of regression line (Table 4.1). This indicated that while males develop longer anterior horns at an earlier age, the growth rate does not then seem to differ between sexes. All of these measurements were correlated.
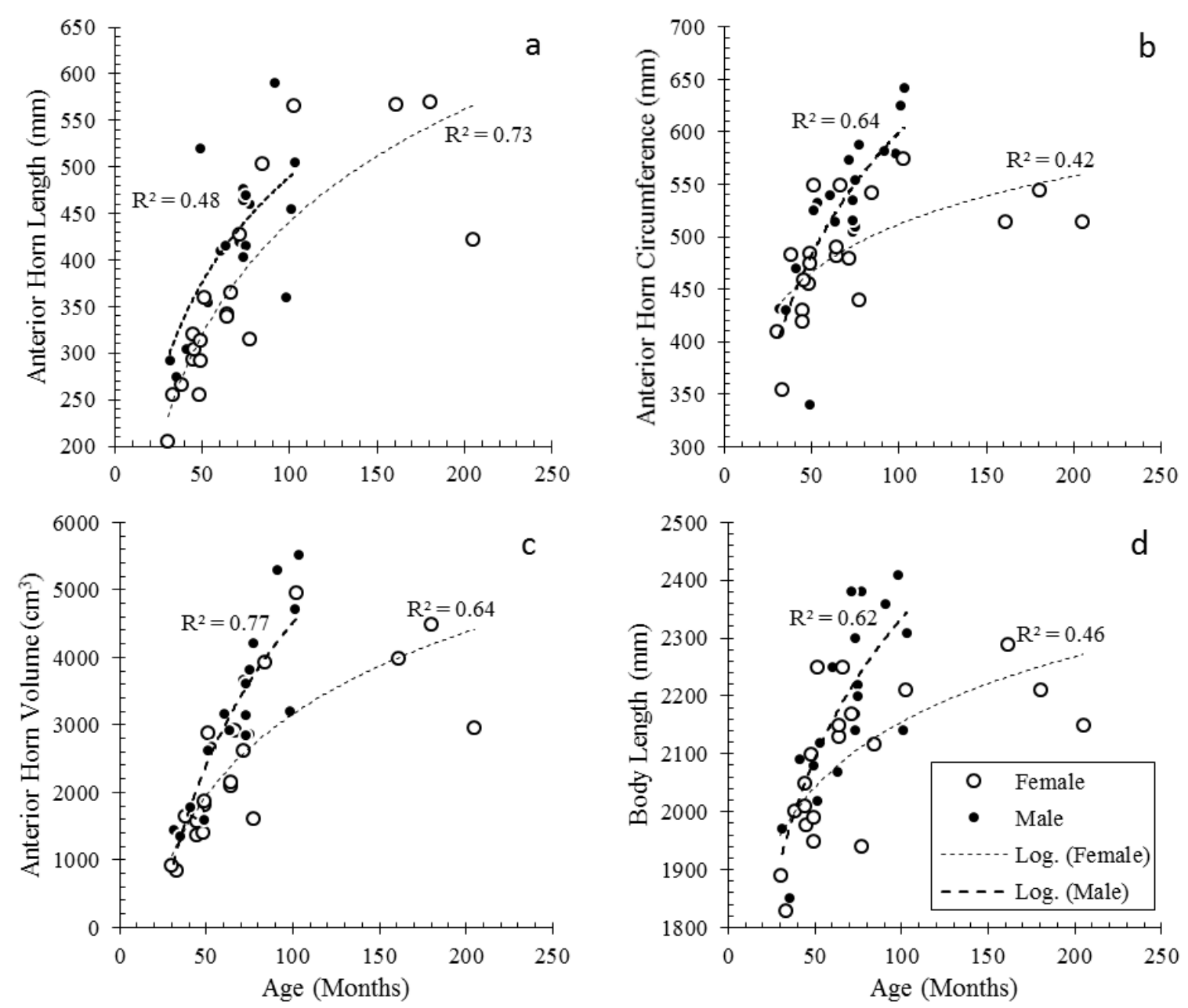

Figure 4.2 Sexual dimorphisms identified in black rhinoceros. The body or horn measurement is plotted against the age of individuals. Lines represent logarithmic trendlines. a) Anterior horn length b) Anterior horn circumference c) Anterior horn volume d) Body length.

\section{Measures of host resources}

I used three measures of body condition. The half girth of an individual (range $950-$ 
$1320 \mathrm{~mm}$ ) was found to be larger by up to $91 \mathrm{~mm}$, or smaller by up to $135 \mathrm{~mm}$ than may be expected by an individual's body length. In addition, the body weight of an individual (range $700-1226 \mathrm{~kg}$ ) was found to be larger by up to $132 \mathrm{~kg}$, or smaller by up to $135 \mathrm{~kg}$ than may be expected by an individual's body length. These two measures of body condition were highly correlated (Pearson correlation $=0.846, \mathrm{p}<0.001, \mathrm{n}=18$, see Figure 4.3). However, the image assessment of body condition was not correlated with either of the measures utilising body measurements (see Figure 4.4). Age and sex had no influence on measures of body condition.

Table 4.1 Sexual dimorphisms in black rhinoceros

\begin{tabular}{lll}
\hline Variable & Intercept & Slope \\
\hline Anterior Horn Length & $\mathrm{F}_{1,36}=7.97 \mathrm{p}=0.01^{*}$ & $\mathrm{~F}_{1,35}=0.53 \mathrm{p}=0.819$ \\
Anterior Horn Circumference & $\mathrm{F}_{1,35}=6.55, \mathrm{p}=0.02^{*}$ & $\mathrm{~F}_{1,35}=8.19, \mathrm{p}=0.01^{*}$ \\
Anterior Horn Volume & $\mathrm{F}_{1,35}=4.27, \mathrm{p}=0.046^{*}$ & $\mathrm{~F}_{1,35}=5.90, \mathrm{p}=0.02^{*}$ \\
Posterior horn length & $\mathrm{F}_{1,36}=0.49, \mathrm{p}=0.49$ & $\mathrm{~F}_{1,35}=3.53, \mathrm{p}=0.07$ \\
Posterior horn circumference & $\mathrm{F}_{1,36}=0.10, \mathrm{p}=0.75$ & $\mathrm{~F}_{1,35}=0.41, \mathrm{p}=0.53$ \\
Posterior horn volume & $\mathrm{F}_{1,36}=1.66, \mathrm{p}=0.21$ & $\mathrm{~F}_{1,35}=2.67, \mathrm{p}=0.11$ \\
Shoulder height & $\mathrm{F}_{1,33}=1.62, \mathrm{p}=0.21$ & $\mathrm{~F}_{1,32}=1.17, \mathrm{p}=0.29$ \\
Half girth & $\mathrm{F}_{1,34}=3.46, \mathrm{p}=0.07$ & $\mathrm{~F}_{1,33}=1.03, \mathrm{p}=0.32$ \\
Body Length & $\mathrm{F}_{1,35}=4.20, \mathrm{p}=0.048^{*}$ & $\mathrm{~F}_{1,35}=5.56, \mathrm{p}=0.02^{*}$ \\
\hline
\end{tabular}

* Denotes significance $(\mathrm{p}<0.05)$

\section{The influence of parasitism on host resources}

No significant impact of parasitism could be found on the size of identified sexual dimorphisms for either sex. Also, none of the measures of body condition were correlated with parasite abundance (see Figures 4.5 and 4.6). Furthermore, the condition or size of sexual dimorphisms was not affected by whether an individual contained a very high intensity of parasite infection.

A power analysis was conducted to investigate the chances of type II error. This assumed that the individuals within which parasites were aggregated also had the lowest growth rates of sexual dimorphisms. In this optimum situation for testing, between 12 and 15 samples would be needed depending on the sexual dimorphism, with volume of the anterior horn needing the fewest samples. 


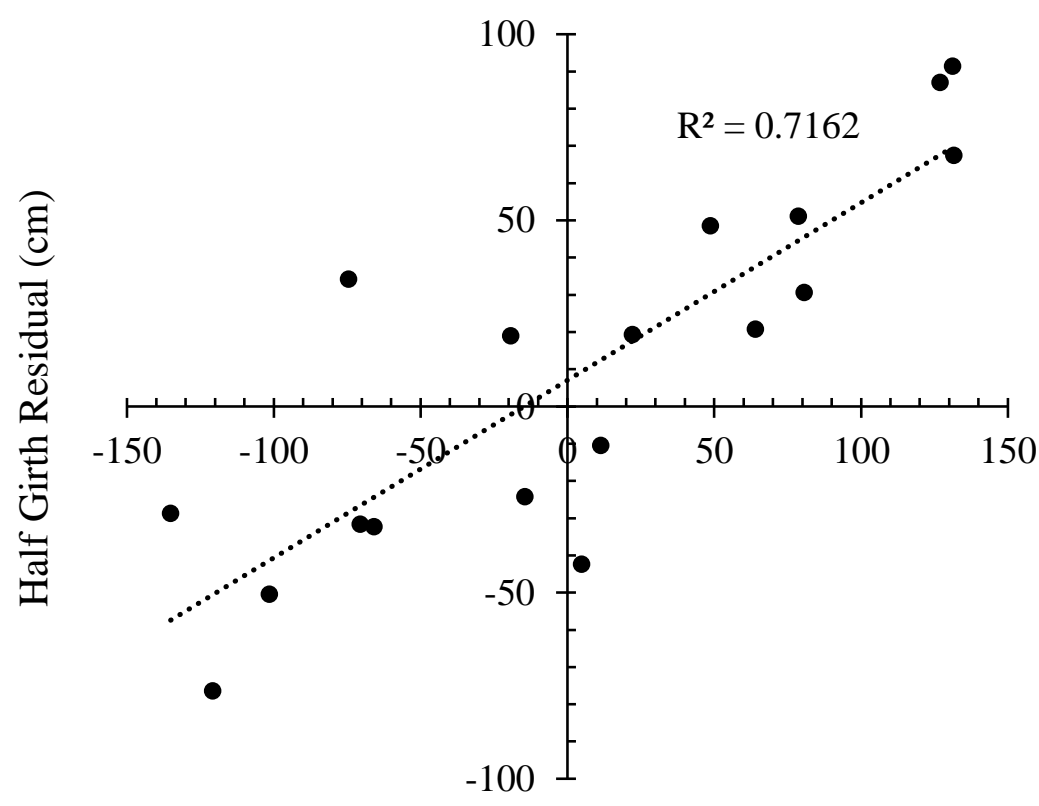

Weight Residual (kg)

Figure 4.3 The correlation between two measures of body condition derived from body measurements - the residuals of the regression of body weight to body length, and half girth to body length.

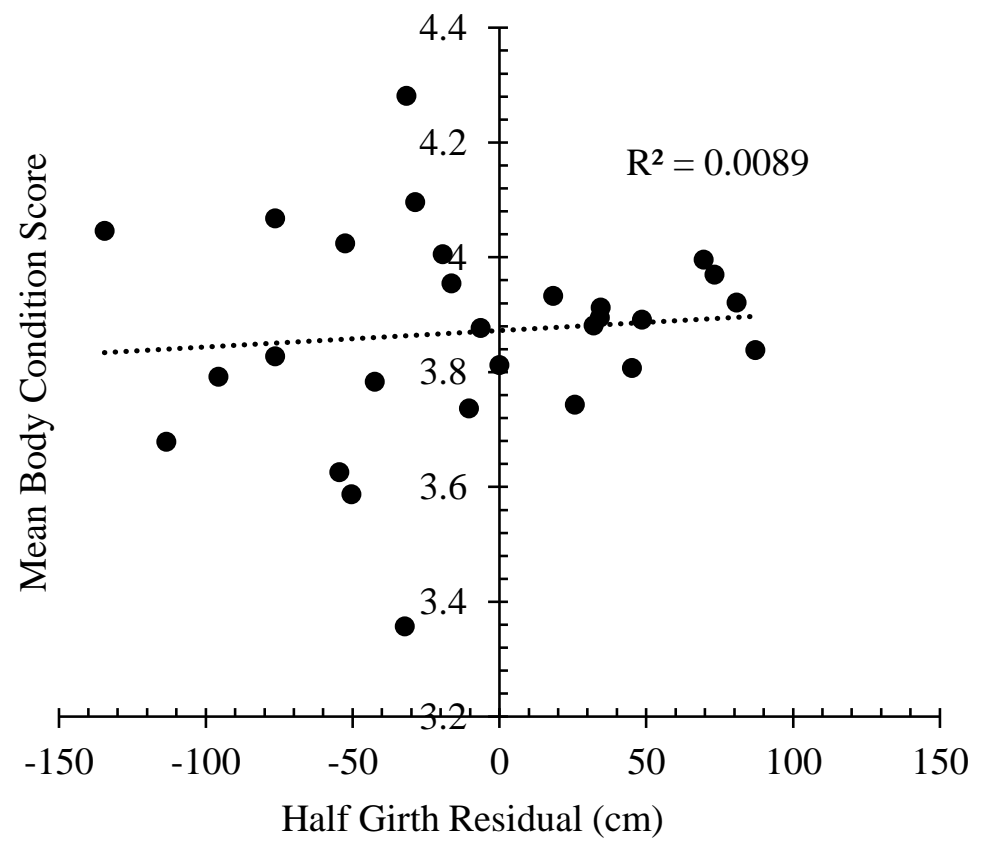

Figure 4.4 The correlation between two measures of body condition - the residuals of the regression of half girth to body length, and a visual assessment of body condition. 
Chapter 4: Individuals
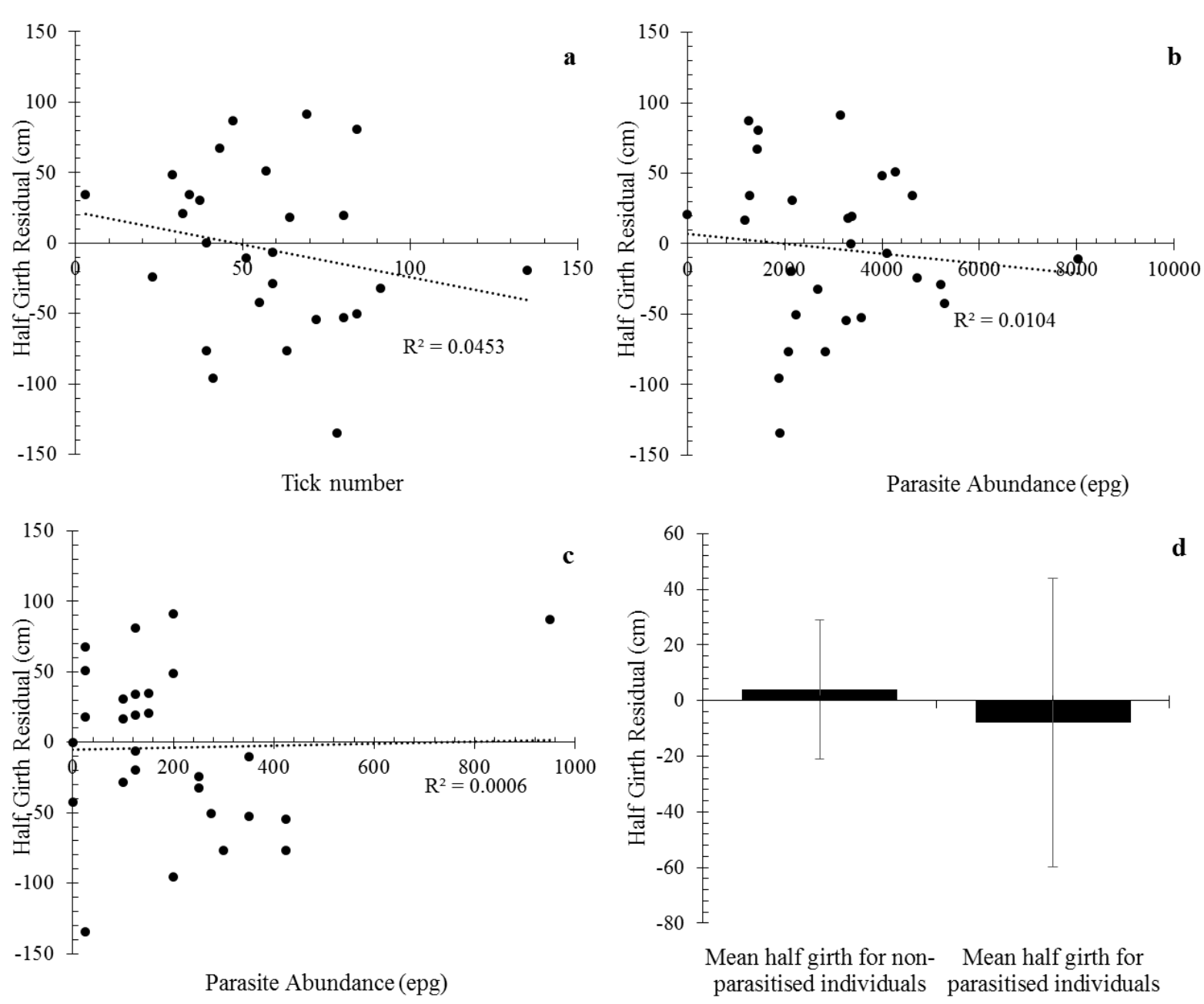

Figure 4.5 The regression between parasite intensity or prevalence and body condition as measured by the residuals from the regression of half girth to body length a) Ticks, b) Strongyles c) Anoplocephala sp. d) Oxyuris sp. (the Scotch Tape Test was used to test only for presence/absence of an infection, not abundance) 

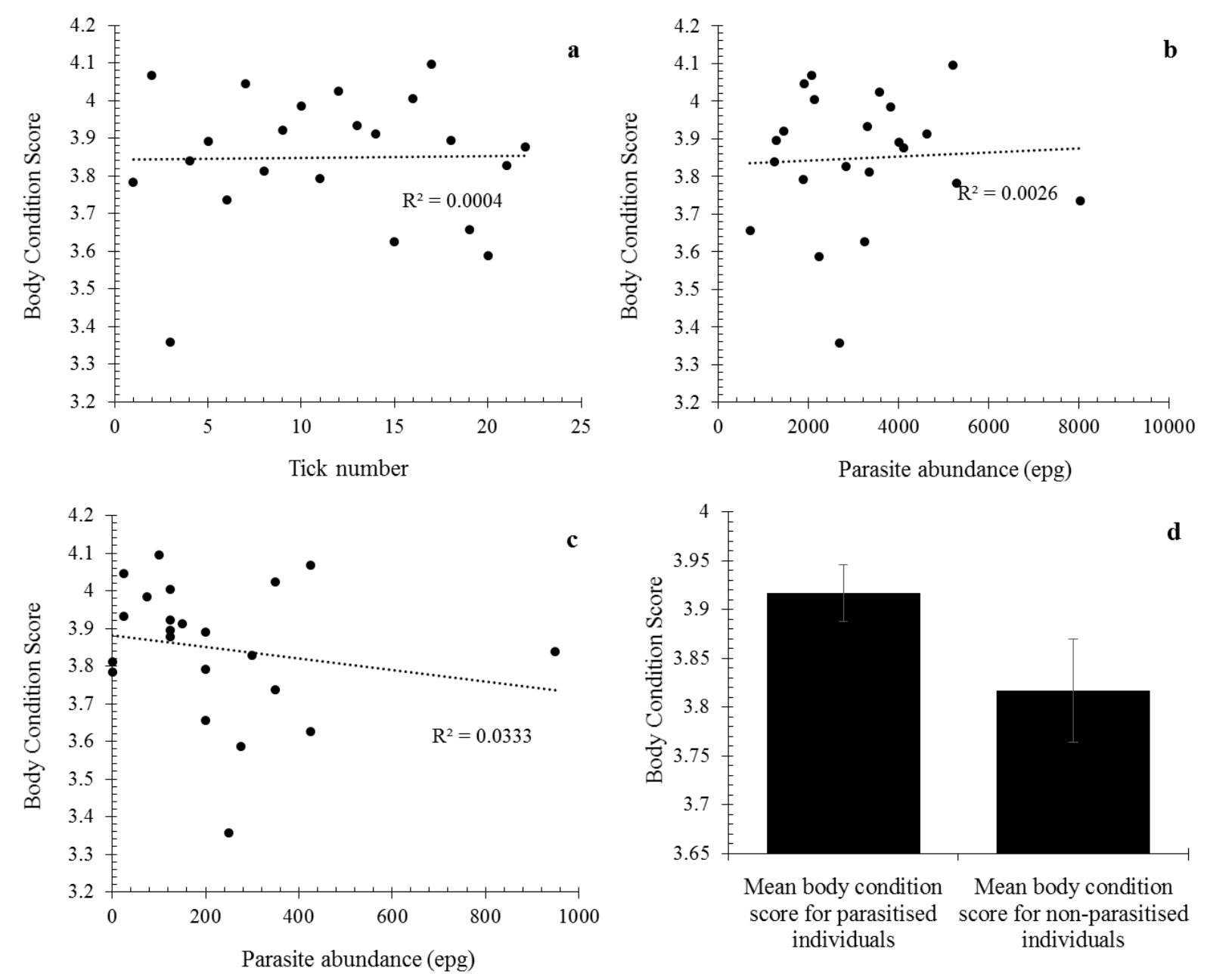

Figure 4.6 The regression between parasite intensity or prevalence and a visual assessment of body condition a) Ticks, b) Strongyles c) Anoplocephala sp. d) Oxyuris sp. (the Scotch Tape Test was used to test only for presence/absence of an infection, not abundance)

\subsection{Discussion}

My data indicate that younger individuals may be more likely to harbor very high parasite intensities. This may indicate that parasite abundance in black rhinoceros peaks between 33 and 63 months old. However, the individuals in my sample were from a limited age group, with the vast majority between 30 and 108 months (2.5-9 years). A larger age range and a greater sample size, especially for males, is needed to accurately describe the pattern of parasitism in black rhinoceros with respect to age. A longitudinal study that tracks parasite abundance as an individual ages would give the best indication of how parasite abundance changes with age. No difference in the abundance of parasites between the sexes could be identified, although there was weak evidence that showed female hosts may have a more aggregated distribution of 
parasites.

Sexual dimorphisms may represent sexually selected characteristics, or be an indication of niche separation between sexes (Krebs and Davies, 1993). Here I found that body length and anterior horns grew faster in males. However, while anterior horn length grew faster in early age, it then seemed to match the growth rate of females (Figure 4.2a). This was reflected in my analysis where the only statistical difference was found in the intercept of regression lines, not in their slope. It would be easy to assume that anterior horn circumference is simply an artefact of body length, however, it is interesting to note that no sexual dimorphisms were found in posterior horn length or circumference. Previous studies have shown that males have larger anterior horns as adults (see 1.5.1). During data collection it was often mentioned by colleagues that female rhinoceros have larger posterior horns. I found no evidence of this, although this may be a trait that becomes apparent only in older adults.

Due to the difficulty of measuring the level of parasite virulence, I here attempted to use a novel method of determining the impacts of parasitism on host resources. It may be expected that even in populations with abundant resources parasites would reduce the amount of excess resources which may be invested in sexually selected characteristics. I found no influence of parasitism on potentially sexually selected traits in the current study. A key issue with this study, predominantly due to sampling and logistic constraints, is small sample size. Hence, when interpreting these results the potential for type II error must be considered. However, power analyses showed that the sample size was sufficient if the parasites studied were the cause of the very slowest growth rates of potentially sexually selected characteristics.

The growth rate of the anterior horn was highly variable, for instance at 50 months of age ( $\pm 1-2$ months) the smallest volume of the anterior horn was $1406 \mathrm{~cm}^{3}$, with the largest over twice the size at $2889 \mathrm{~cm}^{3}$. Here, I failed to explain these differences between individuals. However, the large variation in this potentially sexually-selected characteristic may be of interest to future studies.

I found no impact of parasitism on measures of host body condition. Studies using experiments where, for example, parasites have been removed from individuals have proved useful in showing that parasites impact body condition (Stien et al., 2002; Hillegass et al., 2010). Also, previous studies using feeding experiments have shown that artificially improved nutrition within individuals does reduce susceptibility to parasites (Hines et al., 2007).

My measurement of parasite abundance was only an indication of current parasite load. 
This single sample may not represent lifetime parasite abundance. In particular as other results from this study show how age may affect parasite abundance. However, certain individuals within a population are thought to be predisposed to a higher parasite abundance (see 1.2.1). Hence, current parasite load may give an indication of lifetime parasite burden (McCallum, 1990). This is important because, although measures of body condition assessed current condition, measures of sexually-selected characteristics were measures of lifetime growth rates of the characteristics. Hence, future studies should focus on longitudinal studies that attempt to determine parasite abundance throughout the developmental years of a rhinoceros may be useful. In contrast, a future study focusing on adults may be able to identify those individuals predisposed to parasitism, but will not be able to capture delays in the development of sexuallyselected characteristics.

A visual assessment of body condition did not correlate with other measures of condition. Due to logistical constraints the visual assessment had to be done while the individuals were lying down. This is not an ideal position as the skin and muscles may be contorted in a nonuniform way, making visual assessments of condition difficult (Reuter and Adcock, 1998). The data presented here supports this as the visual assessment did not correlate with other measures of body condition. The visual assessment of condition of animals lying down would not be recommended for future studies.

My study animals were predominantly younger animals, and a cohort translocated from a study reserve that contained an expanding and successful black rhinoceros population. My study was not, therefore, representative of that population or black rhinoceros populations generally. The population is thought to have abundant resources due to a low age at first calf and small inter-calving intervals (Law et al., 2013). Abundant resources may allow black rhinoceros individuals in this population to compensate for the resources lost to high parasite abundance. Indeed the expanding nature of this population, despite its very high abundance of parasites, indicates that parasites are not regulating population size here. However, the age at first reproduction is increasing in this population and this is thought to be a first indication that a population's growth is being limited (Law et al., 2013).

I only tested for the effects of a small proportion of total parasite biomass. Hence, effects may be masked by other unstudied parasite groups, or the potentially combined effects of polyparasitism. A study investigating a wider range of parasites, perhaps including blood parasites, may reveal further the impacts of parasitism. Future studies could also investigate 
whether parasites have a direct effect on measures of fecundity directly relevant to conservation actions- such as when individuals are under stress, pregnancy, calf survival, inter-calving interval, age at first calf, and breeding longevity. 


\section{Chapter 5: Everything in moderation: Principles of parasite control for}

\section{wildlife conservation}

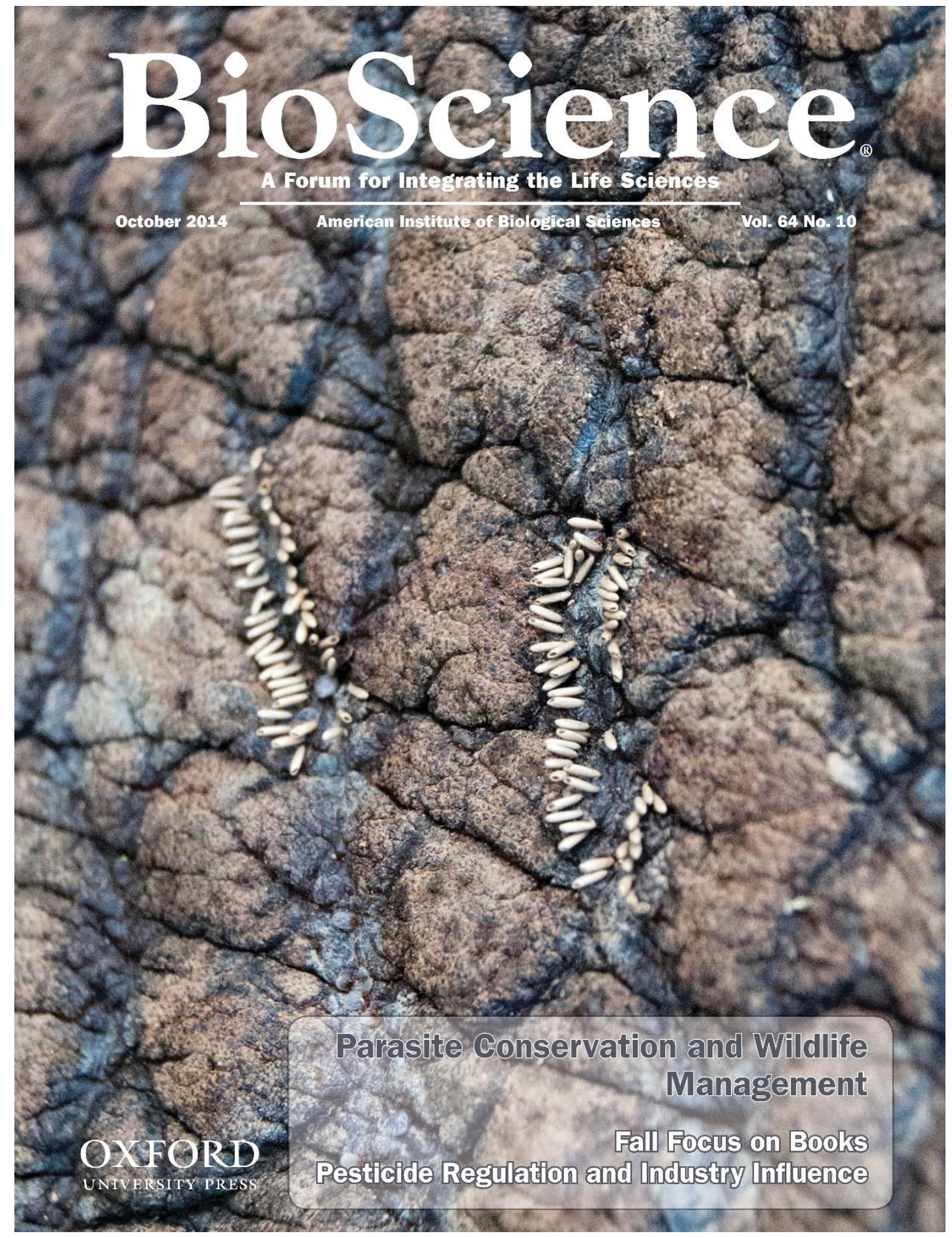

"As to the tongue of the Rhinoceros... it is so rough as to be capable of rubbing a Man's flesh from his Bones" J. Parsons (1743) 


\subsection{Preface}

The thesis so far has focused on the determinants of parasite abundance, useful for predicting where the impacts of parasitism may be greatest, and the impacts of parasites on hosts. While these chapters may be useful investigations, they do not attempt to discuss how they might be applied within conservation management, or indeed the higher level question of whether parasitism should be controlled in threatened hosts. As threatened hosts may contain threatened parasites, this question is clearly worthy of debate and investigation. There are currently no guidelines for the treatment of parasites in host species with conservation status. The following chapter attempts to identify the principles behind any parasite control strategy within threatened hosts.

This chapter has been published, Stringer A.P. \& Linklater W.L. 2015. BioScience, 64, 932-937, DOI: 10.1093/biosci/biu135, and is available in Appendix 4. The text is the final text that was submitted to publishers. Larger additions from the published version for this dissertation are enclosed as footnotes, but small changes have been incorporated into the text. 


\subsection{Abstract}

Parasites can reduce host body condition, impair reproduction, and cause mortality. However, parasites are a major source of biodiversity, a fundamental component of a healthy ecosystem, and could be the group most impacted by the modern-day biodiversity crisis. Parasite control may cause immunological naivety, unbalance parasite-mediated apparent competition between hosts, and destabilize the host-parasite arms race. Here we rank parasite control strategies according to their potential impact on ecosystems. We argue that the need for parasite control increases as the threat a parasite poses to the survival of the host species increases, and hence the justification for control measures that may impact on ecosystems also increases. However, as host-specific parasites may be more endangered than their hosts there is often the need for active parasite conservation such as establishing parasite refugia. Although the principles proposed here are predominantly intuitively obvious, there are numerous examples where they have not been applied. 


\subsection{Introduction}

The co-extinction of dependent species, such as mutualists, commensals and parasites, may be the greatest, yet least appreciated, source of current biodiversity decline (Dunn et al., 2009). Parasites may be a particularly influential component of biodiversity as they act as evolutionary drivers, including effects on host genetic and phenotypic diversity, secondary sexual traits, and community structure (Gómez and Nichols, 2013; Nunn et al., 2004). However, parasites can also be a danger to threatened species due to their impacts on populations (Heard et al., 2013). Furthermore, host inbreeding, environmental pollution, over-exploitation, habitat modification and loss, climate change, contact with domestic livestock, and invasive species may all increase the potential for parasites to do harm (Lafferty and Gerber, 2002; Smith et al., 2009a).

Conservation actions often do not take into account the importance of parasites. For instance, translocation programs often do not acknowledge the parasite bottleneck that they cause, that restrict parasite biodiversity within reintroduced populations (Moir et al., 2012). Indeed, grey wolves (Canis lupus) reintroduced to Yellowstone National Park were treated with a broad-spectrum antiparasitic before release (Almberg et al., 2012). Active parasite conservation strategies are rare (Gómez and Nichols, 2013) and conservation management in the past has led directly to parasite extinctions. For instance, black-footed ferrets (Mustela nigripes) have a depauperate ectoparasite fauna thought to be caused by previous conservation actions (Harris et al., 2014). Also, the extinct California condor louse (Colpocephalum californici) was deliberately killed during the captive breeding of its host the Californian condor (Gymnogyps californianus) (Koh et al., 2004).

Guidelines for the control of parasites in threatened hosts are limited or absent (Woodroffe, 1999). Wildlife parasite control programs have been both successful, clearly reducing the impacts of epidemics (Knobel et al., 2008), and damaging, by having detrimental unintended side-effects on hosts (Burrows et al., 1995). Here we outline the arguments for and against parasite control. We then propose some principles of parasite control in threatened host species that attempt to balance the value of parasite biodiversity and ecosystem roles with the need to protect species of conservation concern.

\subsection{Reasons for parasite control}

Parasites can cause severe population declines. For instance, between 1990 and 1992 the rabies virus was thought to be the cause of a $\sim 69 \%$ decline in the Bale Mountain Ethiopian Wolf (Canis simensis) population (Randall et al., 2004). Many parasites do not directly cause mortality, but do 
reduce body condition, growth, fecundity, or exacerbate pre-existing threats to hosts such as predation or winter survival (Irvine, 2006). These effects impact on population performance and can put populations at risk. For instance, the intestinal nematode Trichostrongylus tenuis is one of the causes of large population fluctuations in its host the red grouse (Lagopus lagopus) (Martínez-Padilla et al., 2014). The influences of parasitism on hosts may also be more cryptic. Schwanz (2008) showed that parasitized female deer mice (Peromyscus maniculatus) produced offspring that were larger in ways that improved offspring fitness, but sacrificed longer-term reproductive potential of the mother.

Threatened species are at particular risk from parasitism (Heard et al., 2013), perhaps because causes of biodiversity loss amplify the parasite burden (Smith et al., 2009a). Also, threatened species are more at risk from inbreeding, which may reduce host immunological defences and resistance (Cassinello et al., 2001). For instance, Acevedo-Whitehouse et al. (2003) showed that inbred Californian sea lions (Zalophus californianus) showed increased incidences of parasitism, and that these individuals may act as reservoirs for further infection.

Climate change is predicted to change the geographic range of both parasites and hosts leading to novel species pairings (Altizer et al., 2013). This will increase the occurrence of emerging infectious diseases which can be devastating. For instance, white-nose syndrome, caused by a psychrophilic fungus (Geomyces destructans), has recently caused a population collapse of brown myotis bats (Myotis lucifugus) in North America, with a mean population decline of $73 \%$ (Frick et al., 2010). Hence, as problems associated with parasitism are expected to grow, strategies to control parasitism will become of increasing importance.

\subsection{Detrimental impacts of parasite control}

Parasites are a fundamental component of a healthy ecosystem and a key link with food webs (Hudson et al., 2006), and as such, the removal of parasites can have numerous unintended consequences. Treatment of a human with antibiotics, for example, can eliminate naturally occurring competitors of the bacteria Clostridium difficile. This allows $C$. difficile to reproduce and spread much more widely in the intestine, causing antibiotic-associated colitis (Johnson and Gerding, 1998). Also, the removal of an endophytic fungus from stout woodreed (Cinna arundinacea) increased host survival, but decreased host population growth due to the effects the fungus has on plant regeneration (Rudgers et al., 2012). 
Parasite control can also affect the resilience of a host to parasitism. If a parasite is eradicated from a population future generations will not have immunological experience of that parasite. If the parasite is reintroduced to the population, naïve individuals could be more vulnerable to it. For instance, translocations of naïve black rhinoceros (Diceros bicornis) into trypanosome (Trypanosoma spp.) endemic areas must be timed with seasonally low trypanosome infection rates to prevent the death of translocated individuals (Mihok et al., 1995).

Parasite control can also affect competition between host species. Parasite-mediated apparent competition occurs when two host species are infected by the same parasite species, but the cost of infection differs between the two hosts. For instance, it is predicted that the control of canine distemper virus in the Serengeti would positively affect both lion (Panthera leo) and cheetah (Acinonyx jubatus) populations. However, lion populations would receive the greater benefit, and denser lion populations would then be directly detrimental to cheetah populations (Chauvenet et al., 2011).

Controlling parasitism may fundamentally disrupt host evolution, as the benefits of control will be biased towards those individuals most detrimentally affected by parasites (Shim and Galvani, 2009). Based on the red-queen hypothesis, the theory that populations must be constantly evolving to remain competitive, the success of parasite-susceptible individuals will potentially create a future population less resistant to parasites (Lively and Dybdahl, 2000). Finally, parasites are a major driving force behind evolution and speciation (Nunn et al., 2004). Hence removing parasites counters one of the overall aims of conservation - to sustain ecological processes including adaptation and evolution.

The benefits of controlling parasites in threatened species, to improve fitness and population performance, then, must be balanced with the individual, ethical and ecosystem costs associated with control. A scheme for targeted parasite control appropriate to the magnitude of the threat a parasite poses, cognisant of wider unintended impacts, and that incorporates the risks to parasite biodiversity, is clearly desirable.

\subsection{A proposal for the principles of parasite control in threatened species}

Here we present a proposal for the principles of parasite control with threatened species:

Principle 1: The greatest need for parasite control is where a host is critically endangered and a parasite greatly affects population performance. This is because the need for parasite control increases as the threat a parasite poses to host-species survival increases. This can be evaluated - as the 
extinction risk of the host (for instance IUCN red list category) and the negative impact of the parasite on host population performance increase, so does the parasite threat. Hence, if a parasite has little effect on population performance and the host is at low risk of extinction then there is little need to control parasitism. In a species of 'least concern' there is no conservation-based argument for parasite control above that required for ecological restoration, unless the species is a reservoir host for parasites of another, more endangered, target species.

Principle 2: As the need for parasite control increases, control strategies with greater impacts on parasite populations and control methods with higher impacts on ecosystems can be justified (see 'Types of parasite control').

Principle 3: For host-specific parasites, as the risk of parasite extinction increases so does the importance of creating parasite refugia and the need for active parasite conservation strategies. Threatened hosts will contain threatened parasites, which are likely to go extinct before a host goes extinct (Altizer et al., 2007). Indeed, a single parasite species may not be found throughout its host's geographic range, and hence a parasite's extinction risk could far exceed its host's. Parasite control will further increase this extinction risk. If the long-term stability of the threatened host species is ensured, parasite refugia can be used to reintroduce lost species and restore natural ecosystem functioning (Moir et al., 2012). Here we assume the precautionary view that parasite host shifts are not linked to the scarcity of hosts (Colwell et al., 2012).

Principle 4: For generalist parasites, parasite control techniques that disrupt a host population's evolutionary response to parasitism should be avoided. This is due to the threat of reinfection - as subsequent reinfections may more detrimentally affect a genetically weakened population. For instance, strategies that increase host population vulnerability by interfering with the host-parasite arms race (such as vaccination and parasite treatment), should only be considered when there are so few hosts left that every individual is important for the survival of the species. Importantly, the control of generalist parasites in threatened hosts will have minimal impacts on overall parasite abundance, as the parasite population is maintained within other reservoir hosts. 
Table 5.1 Different parasite control strategies are classified into one of three broad categories: — - Ecological Restoration, strategies, - Evolutionarily disruptive strategies. The mechanism refers to how either parasite abundance or burden is affected.

\begin{tabular}{|c|c|c|c|}
\hline Type of parasite control & Mechanism & Notes & References \\
\hline Pollution reduction & $\begin{array}{l}\text { Reduce exposure to pollutants that may } \\
\text { affect host immune system functioning. }\end{array}$ & Ecological restoration & (Smith et al., 2009a) \\
\hline $\begin{array}{l}\text { Buffer zone between wildlife and } \\
\text { livestock }\end{array}$ & $\begin{array}{l}\text { Reduce transmission between domestic } \\
\text { livestock and wildlife. }\end{array}$ & Ecological restoration & $\begin{array}{l}\text { (Pedersen et al., } \\
2007)\end{array}$ \\
\hline Livestock vaccination & $\begin{array}{l}\text { Reduce susceptible host density (of } \\
\text { reservoir) and hence parasite abundance. }\end{array}$ & Ecological restoration & $\begin{array}{l}\text { (Pedersen et al., } \\
2007)\end{array}$ \\
\hline Reintroduce parasitic competitors & $\begin{array}{l}\text { Increased competition with the pathogenic } \\
\text { parasite species to reduce its abundance. }\end{array}$ & Ecological restoration & (Lello et al., 2004) \\
\hline $\begin{array}{l}\text { Host translocation in, improve } \\
\text { connectivity between populations. }\end{array}$ & $\begin{array}{l}\text { Reduce inbreeding depression and hence } \\
\text { host susceptibility to parasitism. }\end{array}$ & Ecological restoration & (Smith et al., 2009a) \\
\hline Remove dead hosts & $\begin{array}{l}\text { Carcases are used for reinfection by some } \\
\text { parasites. }\end{array}$ & $\begin{array}{l}\text { Ecologically invasive - removes key link in food } \\
\text { web. }\end{array}$ & (Gates et al., 1995) \\
\hline Host translocation out & $\begin{array}{l}\text { Reduce susceptible host density and hence } \\
\text { abundance. }\end{array}$ & $\begin{array}{l}\text { Ecologically invasive - reduce ecosystem roles of } \\
\text { host. }\end{array}$ & (Wobeser, 2002) \\
\hline Cull or Vaccinate sympatric hosts & $\begin{array}{l}\text { Reduce susceptible host density (of } \\
\text { reservoir) and hence abundance. }\end{array}$ & $\begin{array}{l}\text { Ecologically invasive - Reduces ecosystem roles or } \\
\text { alters host-parasite evolution in sympatric host. }\end{array}$ & (Gurnell et al., 2006) \\
\hline $\begin{array}{l}\text { Reduce vector population through } \\
\text { habitat modification or chemical } \\
\text { trapping. }\end{array}$ & $\begin{array}{l}\text { Reduces transmission rate of parasite and } \\
\text { hence abundance. }\end{array}$ & $\begin{array}{l}\text { Ecologically invasive - reduces vector ecosystem } \\
\text { roles and increases likelihood of local vector } \\
\text { extinction. }\end{array}$ & $\begin{array}{l}\text { (Fyumagwa et al. } \\
2007)\end{array}$ \\
\hline Vaccination & $\begin{array}{l}\text { Reduce susceptible host density, directly } \\
\text { protect threatened species. }\end{array}$ & Alter host-parasite evolution & (Woodroffe, 1999) \\
\hline Treatment & $\begin{array}{l}\text { Eliminate parasite from treated hosts } \\
\text { (although not in all situations - see text). }\end{array}$ & $\begin{array}{l}\text { Alter host-parasite evolution, impacts on non-target } \\
\text { parasite species. }\end{array}$ & $\begin{array}{l}\text { (Spratt, 1997; McGill } \\
\text { et al. 2010) }\end{array}$ \\
\hline
\end{tabular}




\subsection{Types of parasite control}

To apply these proposed principles, it is important to identify how different types of parasite control differ in their impacts on individuals and ecosystems. This will allow conservation managers to choose parasite control strategies that are proportionate to the threat a parasite poses to host extinction. Hence, in Table 5.1 we attempt to broadly rank parasite control strategies by their potential detrimental impacts on ecosystems and host populations.

The least damaging forms of parasite control are closest in principle to ecological restoration. For instance, reducing inbreeding depression aims to restore the ability of a host population to evolve in response to parasite pressure. Ecologically invasive strategies, in contrast, alter ecosystem functioning to decrease the parasite burden of a host population. For instance, habitat may be modified to reduce the abundance of a parasite's vectors. The final grouping contains strategies that are potentially the most damaging forms of parasite control. These are ecologically invasive and, in particular, directly interfere with the host-parasite arms race. For instance, vaccination of hosts may increase the reproductive success of parasitesusceptible hosts (Shim and Galvani, 2009). This may lead to a generation being more susceptible to parasitism, akin to breeding a generation of antelope which cannot outrun a common predator. Furthermore, vaccination may alter parasite evolution, leading to more virulent strains of the parasite (Gandon et al., 2003).

Although we have broadly ranked control strategies by their potential to detrimentally affect ecosystems and host populations, we have not incorporated the intensity, or dose rate, of any proposed control. Low intensity control programs may reduce the detrimental effects of control, as they reduce the parasite burden without causing local parasite extinction (McGill et al. 2010). Also, different parasite control techniques may have differing degrees of impact depending on the parasite group targeted. For instance, vaccination programs that target geographic corridors of transmission (Haydon et al., 2006), or that target vulnerable or important individuals, not only limit the effects of control, but also leave a portion of a population to serve as sentinels for future disease detection ${ }^{\dagger \dagger}$.

\footnotetext{
$\uparrow$ Furthermore, vaccinating only a proportion of a population leaves the rest of the population to evolve in response to the parasitism. Hence, host resistance could develop, the host-parasite arms race continues, and a key argument against the use of vaccination is reduced.
} 
No single parasite control method will be effective for all parasites. For instance, many parasites do not have reservoir hosts in either wildlife or livestock, or use intermediate hosts. Also, reducing host density may only reduce parasite abundance where abundance is determined by density-dependent, rather than frequency-dependent transmission.

\subsection{Past examples of parasite control}

The literature shows that many past examples of parasite control have been in critically endangered species, with parasites that cause mortality. The most common form of parasite control in these situations is vaccination (Woodroffe, 1999; Wobeser, 2002). These examples broadly concur with our proposed principles. However, there are a number of examples in the literature that do not.

African buffalo (Syncerus caffer) are listed in the IUCN red list as a species of least concern. In South Africa, a recent government-funded program created a number of 'disease free' buffalo populations. The populations have been bred to be free from two introduced parasites (Mycobacterium bovis and Brucella abortus) and two native parasites (Aphthae epizootica, the cause of foot-and-mouth disease, and Theileria parva, the cause of corridor disease). The justification for this program was specifically to "save the large gene pool of the Kruger Park buffalo" (Laubscher and Hoffman, 2012). Using the principles proposed here, there is no need to control a parasite with low levels of virulence in a host species of least concern beyond ecological restoration. We also argue that the large gene pool of the Kruger Park buffalo population provides excellent conditions for the co-evolution of buffalo with their parasites. The eradication of parasites from new populations of buffalo may have a range of ecological consequences. For instance, T. parva within cattle often causes fatal disease; the naivety of new populations of buffalo to T. parva may mean they suffer similar serious disease due to a lack of immunity.

Bighorn sheep (Ovis canadensis) are listed in the IUCN red list as a species of least concern. In the 1970s the broad spectrum anthelmintic Fenbendazole was used to treat populations of bighorn sheep for the lungworm Protostrongylus spp. It was proposed that the native lungworm was making $O$. canadensis more susceptible to bacterial pneumonia. The fundamental thinking behind this control program clearly differs from our proposed principles. The use of a broad spectrum anthelmintic, in a host species of least concern, to purportedly control a parasite through an unfounded mechanism (Miller et al., 2000), shows scant regard 
for the value of parasites within an ecosystem. Reducing the transmission rate of bacterial pneumonia between livestock and wildlife, for instance through the use of buffer zones, might have been wiser.

African Elephant (Loxodonta africana) are listed in the IUCN red list as vulnerable to extinction. An estimated 7500 elephants live in the Kruger National Park in South Africa. Between 1987 and 1994 there was an outbreak of encephalomyocarditis virus that caused the death of 64 individuals (Grobler et al., 1995). This native parasite is thought to be predominantly carried by myomorph rodents. A vaccine was developed with the specific aim of protecting elephants from outbreaks of the parasite (Hunter et al., 1998). Using the principles proposed here, there may be a need to control those parasites which have a very high impact on host mortality. However, this single outbreak over a long period may reflect the normal evolution of the host and does not threaten long term population stability. Vaccination may exacerbate the susceptibility of elephants to the parasite, as it will create a population less resistant to encephalomyocarditis virus without controlling the parasite itself (which is maintained primarily within rodents). In the event of a larger outbreak that is of concern, the control or vaccination of the rodent population may be more prudent.

These three examples demonstrate an over-estimation of the threat of parasites, and an under-estimation of the importance of parasites within an ecological community and the value of parasite biodiversity. In contrast there are some management plans which actively think about parasite conservation. For instance, the black rhinoceros in east Africa is recolonizing much of its former range. However, there were concerns that the host-specific black rhinoceros bot fly, Gyrostigma pavesii, was not recolonizing certain populations. The Kenyan black rhinoceros management plan of 1993 proposed translocating black rhinoceros infected with the parasite into areas where it had not recolonized (Brett, 1993). This is an example of active parasite conservation - reintroducing parasites from parasite refugia into newly established host populations.

\subsection{In conclusion}

This chapter aims to provide a rational and robust method to deciding when and how to control parasitism in threatened species, that we believe will help conservation managers avoid unwanted outcomes. The proposed principles will allow reserve managers to tailor parasite control strategies to their own populations. This is important because a parasite's impact on 
population performance may change depending on the strain of the parasite (Lively and Dybdahl, 2000), and hosts may be more susceptible in certain populations due to nutritional or physiological stress (Cornet et al., 2014). This chapter actively encourages the discussion surrounding the parasites of threatened species, because we believe there are numerous avenues for further debate. For instance, these principles do not incorporate the phylogenetic distinctness of the host or parasite species (Faith et al., 2004). They also rely on the IUCN red list which is currently highly biased towards vertebrates (Whiteman and Parker, 2005). Further experimental research is needed on the potentially detrimental effects of controlling parasitism, for example, by leaving a subset of host populations as control populations, free from intervention. 


\section{Chapter 6: Discussion}

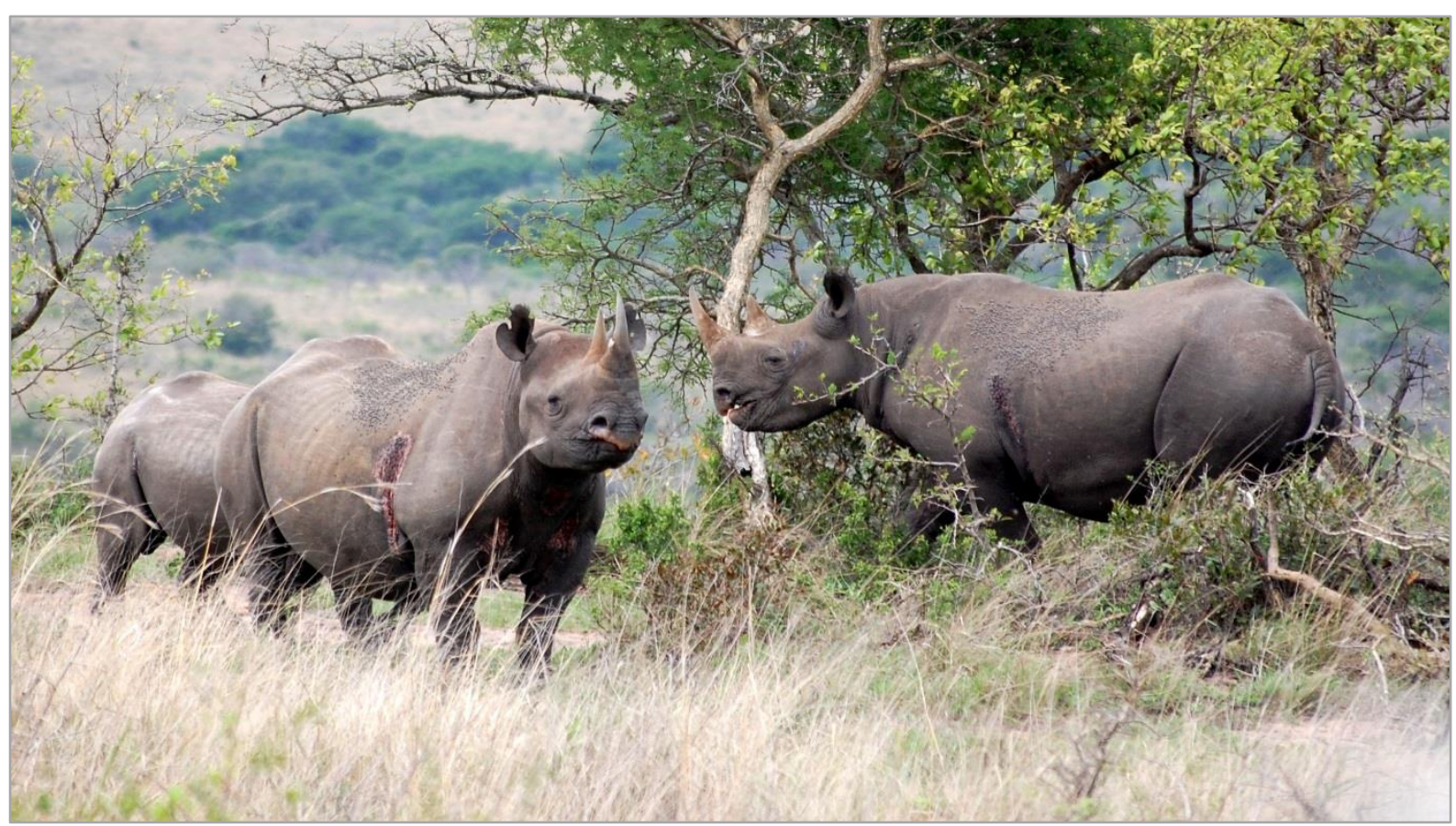

Filarial nematode lesions on black rhinoceros from Hluhluwe-Imfolozi Park, Sep 2009, (C) Andrew Stringer.

"I have never met a rhinoceros that isn't grumpy, irritable and bad-tempered, so no, I don't like rhinos.” Wayne L. Linklater (2012) 
In this study I have combined veterinary, parasitological, and wildlife conservation disciplines to investigate parasite ecology and parasite impacts in a secretive endangered host population. My study advances a range of previous work on a variety of topics - reducing methodological constraints, testing ecological hypotheses, testing for associations between parasite burden and host resources, and finally discussing the importance and value of parasite conservation and how we may balance the sometimes competing priorities of biodiversity conservation.

\subsection{Improving methodologies}

Parasite enumeration in free-ranging wildlife is difficult, time-consuming and costly. Indeed a key restriction to parasitological studies may be the logistical problem of acquiring reliable diagnostic information. To investigate the parasites of threatened host species, noninvasive techniques, such as faecal egg counts, are often essential. In Chapter 2, I tackled some key sources of sampling error for faecal egg counts, with the specific aim of improving the feasibility and cost-effectiveness of parasite enumeration strategies. Black rhinoceros are a species that is difficult to see in the wild, and even more difficult to see defecating. I tackled various factors, such as collecting aged faecal samples and estimating optimal sample size, which will allow researchers to optimise sampling strategies. The chapter opens up new avenues of research on the parasites of rare and elusive wildlife, where little research has been possible or feasible previously. In particular, the non-invasive enumeration of parasites in black rhinoceros was simply not possible on a large scale previous to this work. The chapter recommended that ten individual samples per population are needed to capture the potential variation in parasite abundance for the helminths studied. A key conclusion of the chapter was that the required sample size will vary depending on the level of parasite aggregation. Hence, the recommendation may change for other parasites and host species, or may be used if the level of aggregation is the same.

Some concerns were not addressed within this study. Of greatest importance is that the correlation between faecal egg counts and actual parasite abundance within black rhinoceros has not yet been tested. If the correlation is poor, this will increase the noise associated with methodological techniques, which will increase the likelihood of type II error. Hence, some of the negative results in this thesis, in particular those with small sample size in Chapter 4 (that 
will be more vulnerable to methodological noise), should be interpreted with caution.

It is hoped that researchers will revisit and expand on this published chapter, allowing parasitological research to be easier and more widespread across host species. For instance, of potential importance, but not tested in this chapter, was the impact of my doing all analysis on site, in the field. This allowed me to avoid problems associated with storage methodologies and delays to sample processing (Dacombe et al., 2007; Lynsdale et al., 2015). The further development of in situ techniques and the improved methodological accuracy that this enables should be the focus of future work - it diminishes sampling constraints and greatly increases the scope of work possible.

\subsection{Testing ecological hypotheses}

Anderson and May (1979) proposed a central tenet of epidemiology - density-dependent transmission. This important mechanism, high host-density leading to increased transmission rates and higher parasite abundance, has been shown in microparasites but support for it amongst macroparasites is contradictory and, at best, weak (Arneberg et al., 1998). This has led to other competing theories as to the determinants of parasite abundance gaining attention. These argue that the implications of free-living life stages or intermediate hosts found in macroparasites negate any impact of density-dependent transmission. In Chapter 3 I demonstrated density-dependent transmission for both directly and indirectly transmitted macroparasites of black rhinoceros. Key to this finding was the use of a novel pan-continental experimental framework, made possible by the translocation of black rhinoceros for species conservation. This evidence of a long-assumed fundamental mechanism of epidemiology nicely illustrates the power of simple models to explain complex ecological systems. Population biologists and community ecologists may use experimental frameworks that result from species management in the future to understand disease-population and community dynamics in the same way I have. Such an approach is necessary when working with rare and critically endangered wildlife but also made possible by conservation management.

A key limitation to the conclusions of this chapter were the small sample sizes achieved from some populations, and that faecal samples could not be assigned to individual rhinoceros. The analysis was structured by specifying population as a random effect to ameliorate those concerns. Nevertheless the added robustness analysis which appears as an Addendum to this chapter also helps to allay these concerns. However, further research on both parasite groups 
would be worthwhile to verify the results, for instance within other host species. Although I used sampling techniques to reduce the chance of pseudo-replication, future studies could apply genetic techniques to assign faecal samples to individuals to eliminate repeated sampling of the same individual entirely.

Sample sizes are a consideration and potential constraint on most studies, especially those on critically endangered and elusive species like black rhinoceros. Although, sample number in this thesis may appear low compared to studies of more common, easier-to-sample species, sampling effort was in fact sufficient to meet objectives, test hypotheses and reach important conclusions. Moreover, sampling effort was also substantial when it is viewed in its conservation context. Forty-one percent of all South African black rhinoceros populations were sampled for this thesis and, if samples were all from different individuals, Chapter 3 would represent $12.5 \%$ of all black rhinoceros in South Africa outside Kruger National Park in 2010 (IUCN, 2013; Ferreira et al., 2011).

White rhinoceros (Ceratotherium simum) are also thought to be a host for Anoplocephala sp. (Penzhorn et al., 1994). However, the addition of white rhinoceros density to the hostdensity model actually weakened the model fit $(\Delta \mathrm{AICc}=1.81)$. This indicates that white rhinoceros may not be a host for the Anoplocephala sp. population within black rhinoceros, and reveals the importance of genetic studies within parasitology. For instance, the Anoplocephala sp. populations between these two hosts may never interact, although using morphological species identification only it would be assumed that the species infected both hosts. A control program based on this false assumption may end up targeting only one of the populations. Genetic analyses to establish whether the tapeworms are different species could be the direction of future work as they have proved useful in the past (Miura et al., 2005).

Genetic studies could also be useful in the future study of strongyle parasites. In this study I grouped all strongyles into a single group, due to a lack of phenotypic variation between eggs of different species. Future research could use genetic techniques to compare and contrast how different species are distributed across South Africa, and whether the small size of some founder populations has led to parasite species bottlenecks. Here I found no evidence that the size of a founder population influenced parasite abundance. However, if strongyles are in close competition, other species may fill the voids left by species not translocated into new populations.

In Chapter 4, I also investigated whether host age or sex had an impact on parasite 
abundance. I found that the peak of parasite abundance within black rhinoceros may be between 33 and 63 months, but further research with a broader spectrum of age ranges is needed to verify the result. Age and sex are predicted to be important from a review of the literature (see 1.2.1). However, numerous other factors may be important in determining individual level parasite abundance. Of particular note, but not tested in this thesis, are the genetic factors that may influence parasite abundance, such as the level of variability in the major histocompatibility complex (Paterson et al., 1998).

\subsection{Detrimental impacts of parasitism}

In Chapter 4 I investigated a range of parasite groups for their impacts on host resources and lifetime growth rates in black rhinoceros. I identified four potentially sexually selected characteristics, but did not find an influence of parasitism on their growth rates or other measures of body condition. There were limitations to the conclusions of this chapter due to small sample size. It was expected at the planning stage that 40 black rhinoceros could be sampled and measured. However, due to logistic difficulties associated with sample collection (a lack of faeces in the rectum and a lack of opportunity to collect from an individual after the capture operation) and measuring (logistic difficulties in weighing animals), sample size was much reduced.

The population I studied was abundant in parasites, but also abundant in food resources (Law et al., 2013). These two factors may be intrinsically linked within black rhinoceros - as food resources increase a more dense population of rhinoceros is possible, leading to a higher abundance of parasites. The impacts of parasites will be greatest when hosts do not have abundant resources, and so have fewer resources to dedicate to immune processes and a small diversion of those resources by parasites is more noticeable. Hence, the distance a black rhinoceros population is from ecological carrying capacity may be a key factor determining host susceptibility. The high transmission rate caused by a high density may also, in evolutionary terms, allow parasites to become more virulent and detrimental to their hosts. This is because, while parasites must find an optimal balance between virulence and host longevity, there may be an association between a high transmission rate and high levels of virulence (Bull, 1994). The influence of all these factors means that there may be an inherent difference between parasite abundance and parasite burden. Parasite burden is the key factor which must be evaluated, as it will reveal the impacts of parasites on a host's population performance, 
essential for the conservation decisions outlined in Chapter 5.

Further research could test for the influence of parasitism in a range of black rhinoceros populations with differing levels of host resources and differing densities - essentially a longitudinal extension of Chapter 4 , which adopts a similar population-level framework to that in Chapter 3. Furthermore, the impacts of parasites on the key conservation metric of lifetime reproductive success should be investigated. Finally, a wider range of parasite species could be studied, for instance the potentially important blood parasites (see 1.6.1), and hence the potential interactive and synergistic impacts of polyparasitism investigated (Keusch and Migasena, 1982).

\subsection{Parasite control in threatened hosts}

The common view of parasitism and disease is almost wholly negative. However, parasites are an essential and abundant component of biodiversity that influence many aspects of ecosystem functioning. Indeed parasite control may have numerous detrimental effects. However in threatened species, in conjunction with other pressures, parasites may be a threat to population survival. How to balance the benefits of parasite ecosystem roles with the need to protect conservation-reliant host species has rarely been discussed and remained unresolved. In Chapter 5, I outlined the positive and negative arguments behind parasite control and discussed the principles behind when and how to control parasitism in threatened species. These principles are based on the threat a parasite poses to host-species survival and lead to recommendations on the types of parasite control that should be used in different situations. Finally I discussed the literature on previous examples of parasite control and compared those strategies to my own recommendations. I tackled a large and complex conservation problem with a logical and robust approach. There was a conscious attempt to promote discussion on a conservation frontier that has rarely been debated previously, in the anticipation that conservationists will raise the importance of parasitism in future debates about parasite control. It is hoped that the logical approach to parasite control taken here will ultimately reduce the chance of parasite extinction due to unneeded parasite control, reduce investment in unnecessary control programs, and also increase the use of less harmful types of control.

The proposed principles are a useful first step, but there are many situations where their application may be difficult or not obvious. For instance, how should a conservation manager approach a situation where parasites are the ultimate cause of mortality, but their level of impact 
or virulence is affected by a proximate cause? It will ultimately be up to the conservation manager to decide how more complex situations will be managed, but I hope that a similarly logical approach can be developed. I also described some of the different types of parasite control. Undoubtedly this is not a comprehensive list. Also, I broadly grouped them by their strength (the level of potential detrimental effects that they may cause); however, the control methods may have different levels of detrimental effects depending on the host-parasite relationship to which they are applied. The principles also do not address situations where a parasite may be commensal in some situations, but highly virulent in others. Hence, there are numerous avenues for further development and discourse in this complex area.

If I apply the principles discussed in Chapter 5 to the parasites investigated within this study, we can see how they might be applied to different situations. Black rhinoceros are a critically endangered species, hence there is a justification for using parasite control where parasites have a moderate negative impact on population performance. Reintroduction success has been identified as a key area where conservation strategies can be improved. Currently approximately $10 \%$ of translocated individuals die within their first year post-release (Linklater et al., 2011). Hence, more vulnerable (Linklater et al., 2012) or more important individuals (i.e. females) could be treated for their parasites at translocation. The targeted aims of parasite control within these individuals could be tailored to the pathogenicity of the parasite to be controlled. This may improve the reintroduction success of these individuals, improving population performance. Furthermore, parasite interactions would still be maintained in the longer term within the new population as parasites would still be translocated within nontreated individuals. This proposal balances the need to maintain parasite diversity and ecosystem roles, with the need to maximise black rhinoceros population performance. It also shows how charismatic mega-fauna can be used directly as umbrella species for wider ecosystem restoration. While the strategy would increase the parasite bottleneck inherently associated with reintroduction programs (Moir et al., 2012), parasite refugia could be used to restore parasite biodiversity in the future. Interestingly, there may already be unintended consequences from the methods used for host capture that control parasitism at translocation, increasing the parasite bottleneck unintentionally (see Appendix 1).

\subsection{Faecal egg counts in other mammalian hosts}

I did not compare the abundance of parasites within black rhinoceros with other 
mammalian hosts, but such a comparison might be interesting, even informative.

Faecal egg counts and faecal oocyst counts have been used with numerous parasite groups such as nematodes, cestodes, coccidia and trematodes (Ocaido et al., 1999; Ezenwa and Jolles, 2008; Krauth et al., 2012). Comparing egg counts between host species is difficult primarily because there will be different species of parasite in different hosts, differing locations of studies, and differing methodologies. However, the strongyle nematodes are found in a wide range of host species. My estimates of mean strongyle FEC within a population ranged from 44-4075 epg. The highest intensity of infection recorded within an individual was 7200 epg. To give some comparison, Ezenwa (2003) surveyed 11 African mammalian herbivores and found a range of mean strongyle abundance estimates, the highest being 2560 epg within Grant's gazelle. However, parasite abundance may be affected by body size or phylogeny (Poulin and George-Nascimento, 2007). To compare similar-sized host species, buffalo are approximately a third smaller than black rhinoceros (Estes, 1991) and show FECs between 30 and 2000 epg (Ezenwa and Jolles, 2008). Elephants (2-5 x larger than black rhino) show FECs between 433 and 2238 (Thurber et al., 2011). The slightly larger but phylogenetically similar white rhinoceros show FECs between 65-1500 epg (Condy et al., 1963). My comparisons may indicate that black rhinoceros have extraordinarily high faecal egg counts. Few conclusions can be drawn from these figures, but it is potentially useful to speculate why black rhinoceros might support such high counts. Some theories predict that parasite abundance may increase in threatened species, which may be due to their increased susceptibility to parasitism - as discussed in Chapter 5. Furthermore, population and geographic bottlenecks, like those found in the populations studied, may lead to parasite extinction. This in turn lowers interspecific parasite competition and increases the remaining parasite species abundance (Altizer et al., 2007). The possibility that the abundance and effects of strongyles in black rhinoceros might be exacerbated due to lowered interspecific competition is an extremely interesting avenue for future research.

\subsection{In conclusion}

I initially set out to investigate the key influences on parasite abundance within black rhinoceros, the effects of parasitism on black rhinoceros and how they can be measured, and debate whether parasites should be controlled within threatened host species. I made an empirical advance in understanding parasite-host relationships in black rhinoceros possible by 
testing and refining sampling protocols and methods. By applying these techniques to the use of an extraordinary experimental design (the replication of the same host-parasite relationship across different regions of South Africa) I believe that the thesis was also successful in identifying the key influence on population level macroparasite abundance - host density. However, the determinants of parasite abundance on an individual level, such as host age and sex, were only briefly investigated, and conclusions were hampered by small sample size. Testing the influence of parasites on host resources was also hampered here by small sample size. The investigation was restricted to a population with high levels of host resources, and future study could attempt to differentiate the key differences between parasite abundance and parasite burden. Finally, I believe I was successful in exploring the larger questions of how best to approach the conservation of a host-parasite relationship and how to balance conflicting conservation priorities.

The relationship between hosts and parasites is likely to be of growing interest for conservationists and biologists. There is large potential to improve techniques of parasite sampling and enumeration, and our understanding of parasite ecology. Parasites are likely to attract increasing conservation interest due to their influential roles within ecosystems as well as being threats to rare hosts. It is hoped the present study will promote debate on this conservation frontier in addition to the improvement in sampling techniques and understanding of host-parasite relationships that it achieved. 


\section{References}

Able, D.J. (1996) The contagion indicator hypothesis for parasite-mediated sexual selection. Proceedings of the National Academy of Sciences of the United States of America, 93, 2229-2233.

Acevedo-Whitehouse, K., Gulland, F., Greig, D. \& Amos, W. (2003) Disease susceptibility in California sea lions. Nature, 422, 35.

Adcock, K. (2009) Status and Management of Black Rhino in Namibia, South Africa and Zimbabwe - January 2005 to December 2006. SADC Rhino Management Group.

Adcock, K. (2014) Visual Assessment of Black Rhino Browse Availability. SADC Regional Programme for Rhino Conservation, Harare.

Albon, S.D., Stien, A., Irvine, R.J., Langvatn, R., Ropstad, E. \& Halvorsen, O. (2002) The role of parasites in the dynamics of a reindeer population. Proceedings of the Royal Society B: Biological Sciences, 269, 1625-1632.

Alizon, S., de Roode, J.C. \& Michalakis, Y. (2013) Multiple infections and the evolution of virulence. Ecology Letters, 16, 556-567.

Alizon, S., Hurford, A., Mideo, N. \& Van Baalen, M. (2009) Virulence evolution and the tradeoff hypothesis: History, current state of affairs and the future. Journal of Evolutionary Biology, 22, 245-259.

Almberg, E.S., Cross, P.C., Dobson, A.P., Smith, D.W. \& Hudson, P.J. (2012) Parasite invasion following host reintroduction: A case study of Yellowstone's wolves. Philosophical Transactions of the Royal Society B: Biological Sciences, 367, 2840-2851.

Altizer, S., Nunn, C.L. \& Lindenfors, P. (2007) Do threatened hosts have fewer parasites? A comparative study in primates. Journal of Animal Ecology, 76, 304-314.

Altizer, S., Nunn, C.L., Thrall, P.H., Gittleman, J.L., Antonovics, J., Cunningham, A.A., Dobson, A.P., Ezenwa, V., Jones, K.E., Pedersen, A.B., Poss, M. \& Pulliam, J.R.C. (2003) Social Organization and Parasite Risk in Mammals: Integrating Theory and Empirical Studies. Annual Review of Ecology, Evolution, and Systematics, 34, 517-547.

Altizer, S., Ostfeld, R.S., Johnson, P.T.J., Kutz, S. \& Harvell, C.D. (2013) Climate change and infectious diseases: From evidence to a predictive framework. Science, 341, 514-519.

Anderson-Lederer, R.M., Linklater, W.L. \& Ritchie, P.A. (2012) Limited mitochondrial DNA variation within South Africa's black rhino (Diceros bicornis minor) population and implications for management. African Journal of Ecology, 50, 404-413.

Anderson, M.R. \& May, R.M. (1991) Infectious Diseases of Humans: Dynamics and Control. Oxford University Press, New York.

Anderson, R.C. (1992) Nematode Parasites of Vertebrates: Their Development and Transmission. C.A.B. International, Oxon.

Anderson, R.M. \& May, R.M. (1979) Population biology of infectious diseases: Part I. Nature, 280, 361-367.

Anderson, R.M. \& May, R.M. (1982) Coevolution of hosts and parasites. Parasitology, 85, 411-426.

Anderson, R.M. \& Schad, G.A. (1985) Hookworm burdens and faecal egg counts: an analysis of the biological basis of variation. Transactions of the Royal Society of Tropical 
Medicine and Hygiene, 79, 812-825.

Arneberg, P., Skorping, A., Grenfell, B. \& Read, A.F. (1998) Host densities as determinants of abundance in parasite communities. Proceedings of the Royal Society B: Biological Sciences, 265, 1283-1289.

Atkinson, J.A.M., Gray, D.J., Clements, A.C., Barnes, T.S., McManus, D.P. \& Yang, Y.R. (2013) Environmental changes impacting Echinococcus transmission: Research to support predictive surveillance and control. Global Change Biology, 19, 677-688.

Balmer, O., Stearns, S.C., Schötzau, A. \& Brun, R. (2009) Intraspecific competition between co-infecting parasite strains enhances host survival in African trypanosomes. Ecology, 90, 3367-3378.

Baudena, M.A., Chapman, M.R., French, D.D. \& Klei, T.R. (2000) Seasonal development and survival of equine cyathostome larvae on pasture in south Louisiana. Veterinary Parasitology, 88, 51-60.

Baylis, H.A. (1939) A new species of Oxyuris (Nematoda) from a rhinoceros. Annals and Magazine of Natural History, 11, 516-524.

Becher, A.M., Mahling, M., Nielsen, M.K. \& Pfister, K. (2010) Selective anthelmintic therapy of horses in the Federal states of Bavaria (Germany) and Salzburg (Austria): An investigation into strongyle egg shedding consistency. Veterinary Parasitology, 171, 116-122.

Beldomenico, P.M., Telfer, S., Gebert, S., Lukomski, L., Bennett, M. \& Begon, M. (2008) Poor condition and infection: A vicious circle in natural populations. Proceedings of the Royal Society B: Biological Sciences, 275, 1753-1759.

Bengis, R.G., Kock, R.A. \& Fischer, J. (2002) Infectious animal diseases: The wildlife/livestock interface. OIE Revue Scientifique et Technique, 21, 53-65.

Bentley, K.W. \& Hardy, D.G. (1967) Novel analgesics and molecular rearrangements in the morphine-thebaine group. 3. Alcohols of the 6,14-endo-ethenotetrahydrooripavine series and derived analogs of $\mathrm{N}$-allylnormorphine and -norcodeine. Journal of the American Chemical Society, 89, 3281-3292.

Biggs, D., Courchamp, F., Martin, R. \& Possingham, H.P. (2013) Legal trade of Africa's Rhino horns. Science, 339, 1038-1039.

Boag, B., Lello, J., Fenton, A., Tompkins, D.M. \& Hudson, P.J. (2001) Patterns of parasite aggregation in the wild European rabbit (Oryctolagus cuniculus). International Journal for Parasitology, 31, 1421-1428.

Bolker, B.M., Brooks, M.E., Clark, C.J., Geange, S.W., Poulsen, J.R., Stevens, M.H.H. \& White, J.S.S. (2009) Generalized linear mixed models: a practical guide for ecology and evolution. Trends in Ecology and Evolution, 24, 127-135.

Boomker, J., Bain, O., Chabaud, A. \& Kriek, N.P.J. (1995) Stephanofilaria thelazioides n. sp. (Nematoda: Filariidae) from a hippopotamus and its affinities with the species parasitic in the African black rhinoceros. Systematic Parasitology, 32, 205-210.

Bordes, F. \& Morand, S. (2009) Coevolution between multiple helminth infestations and basal immune investment in mammals: Cumulative effects of polyparasitism? Parasitology Research, 106, 33-37.

Bowman, D.B. (2013) Georgis' Parasitology for Veterinarians. 10th edn. Saunders, 
Philadelphia.

Bracke, M.B.M. (2011) Review of wallowing in pigs: Description of the behaviour and its motivational basis. Applied Animal Behaviour Science, 132, 1-13.

Brett, R.A. (1993) Conservation strategy and management plan for the black rhinoceros (Diceros bicornis) in Kenya. Kenya Wildlife Service, Nairobi.

Brocklesby (1965) Some parasites of East African wild animals. East African Wildlife Journal, 3, 120-122.

Brown, S.P. \& Grenfell, B.T. (2001) An unlikely partnership: Parasites, concomitant immunity and host defence. Proceedings of the Royal Society B: Biological Sciences, 268, $2543-$ 2549.

Brunsdon, R.V. (1971) Trichostronglye worm infection in cattle: further studies on problems of diagnosis and on seasonal patterns of occurrence. New Zealand Veterinary Journal, 19, 203-212.

Bryan, R.P. \& Kerr, J.D. (1989) The relation between the natural worm burden of steers and the faecal egg count differentiated to species. Veterinary Parasitology, 30, 327-334.

Buchmann, K. \& Lindenstrøm, T. (2002) Interactions between monogenean parasites and their fish hosts. International Journal for Parasitology, 32, 309-319.

Buckling, A. \& Brockhurst, M.A. (2008) Kin selection and the evolution of virulence. Heredity, 100, 484-488.

Bull, J.J. (1994) Virulence. Evolution, 48, 1423-1437.

Burrows, R., Hofer, H. \& East, M.L. (1995) Population dynamics, intervention and survival in African wild dogs (Lycaon pictus). Proceedings of the Royal Society B: Biological Sciences, 262, 235-245.

Bush, A.O., Lafferty, K.D., Lotz, J.M. \& Shostak, A.W. (1997) Parasitology meets ecology on its own terms: Margolis et al. revisited. Journal of Parasitology, 83, 575-583.

Cabaret, J., Gasnier, N. \& Jacquiet, P. (1998) Fecal egg counts are representative of digestivetract strongyle worm burdens in sheep and goats. Parasite 5, 137-142.

Cabaret, J., Mage, C. \& Bouilhol, M. (2002) Helminth intensity and diversity in organic meat sheep farms in centre of France. Veterinary Parasitology, 105, 33-47.

Campbell, R.D., Rosell, F., Nolet, B.A. \& Dijkstra, V.A.A. (2005) Territory and group sizes in Eurasian beavers (Castor fiber): Echoes of settlement and reproduction? Behavioral Ecology and Sociobiology, 58, 597-607.

Carstensen, H., Larsen, L., Ritz, C. \& Nielsen, M.K. (2013) Daily Variability of Strongyle Fecal Egg Counts in Horses. Journal of Equine Veterinary Science, 33, 161-164.

Carval, D. \& Ferriere, R. (2010) A unified model for the coevolution of resistance, tolerance, and virulence. Evolution, 64, 2988-3009.

Cassinello, J., Gomendio, M. \& Roldan, E.R.S. (2001) Relationship between coefficient of inbreeding and parasite burden in endangered gazelles. Conservation Biology, 15, 11711174.

Cattadori, I.M., Boag, B., Bjørnstad, O.N., Cornell, S.J. \& Hudson, P.J. (2005) Peak shift and epidemiology in a seasonal host-nematode system. Proceedings of the Royal Society B: Biological Sciences, 272, 1163-1169.

Cattadori, I.M., Haydon, D.T. \& Hudson, P.J. (2005) Parasites and climate synchronize red 
grouse populations. Nature, 433, 737-741.

Chauvenet, A.L.M., Durant, S.M., Hilborn, R. \& Pettorelli, N. (2011) Unintended consequences of conservation actions: Managing disease in complex ecosystems. PLoS $O N E, 6$, e28671.

Chiejina, S. N., Fakae, B. B., Behnke, J. M., Nnadi, P. A., Musongong, G. A. \& Wakelin, D. (2002) Expression of acquired immunity to a local isolate of Haemonchus contortus by the Nigerian West African Dwarf goat. Veterinary Parasitology 104, 229-242.

Christensen, C.M., Barnes, E.H., Nansen, P., Roepstorff, A. \& Slotved, H.C. (1995) Experimental Oesophagostomum dentatum infection in the pig: Worm populations resulting from single infections with three doses of larvae. International Journal for Parasitology, 25, 1491-1498.

Claerebout, E. \& Vercruysse, J. (2000) The immune response and the evaluation of acquired immunity against gastrointestinal nematodes in cattle: a review. Parasitology, 120, S25-S42.

Clausen, B. (1981) Survey for trypanosomes in black rhinoceros (Diceros bicornis). Journal of Wildlife Diseases, 17, 581-586.

Clutton-Brock, T.H. \& Vincent, A.C.J. (1991) Sexual selection and the potential reproductive rates of males and females. Nature, 351, 58-60.

Coadwell, W.J. \& Ward, P.F. (1982) The use of faecal egg counts for estimating worm burdens in sheep infected with Haemonchus contortus. Parasitology, 85, Pt 2/.

Cogley, T.P. (1990) Morphology of the eggs of the rhinoceros bot flies Gyrostigma conjungens and G. pavesii (Diptera : Gasterophilidae). International Journal of Insect Morphology and Embryology, 19, 323-326.

Collyer, M.L. \& Stockwell, C.A. (2004) Experimental evidence for costs of parasitism for a threatened species, White Sands pupfish (Cyprinodon tularosa). Journal of Animal Ecology, 73, 821-830.

Coltman, D.W., Pilkington, J.G., Smith, J.A. \& Pemberton, J.M. (1999) Parasite-mediated selection against inbred Soay sheep in a free-living, island population. Evolution, 53, 1259-1267.

Colwell, R.K., Dunn, R.R. \& Harris, N.C. (2012) Coextinction and persistence of dependent species in a changing world. Annual Review of Ecology, Evolution, and Systematics, 43, 183-203.

Condy, J.B., MacCulloch, J.I.M., Rodger, J.O.K. \& Thomson, J.W. (1963) The treatment of eight square-lipped rhinoceros (Ceratotherium simum) with an anthelmintic. Journal of the South African veterinary medicine Association, 31, 99-101.

Cornet, S., Bichet, C., Larcombe, S., Faivre, B. \& Sorci, G. (2014) Impact of host nutritional status on infection dynamics and parasite virulence in a bird-malaria system. Journal of Animal Ecology, 83, 256-265.

Craig, B.H., Pilkington, J.G. \& Pemberton, J.M. (2006) Gastrointestinal nematode species burdens and host mortality in a feral sheep population. Parasitology, 133, 485-496.

Cringoli, G., Rinaldi, L., Veneziano, V., Capelli, G. \& Scala, A. (2004) The influence of flotation solution, sample dilution and the choice of McMaster slide area (volume) on the reliability of the McMaster technique in estimating the faecal egg counts of 
gastrointestinal strongyles and Dicrocoelium dendriticum in sheep. Veterinary Parasitology, 123, 121-131.

Cunningham, A.A. (1996) Disease risks of wildlife translocations. Conservation Biology, 10, 349-353.

Dacombe, R.J., Crampin, A.C., Floyd, S., Randall, A., Ndhlovu, R., Bickle, Q. \& Fine, P.E.M. (2007) Time delays between patient and laboratory selectively affect accuracy of helminth diagnosis. Transactions of the Royal Society of Tropical Medicine and Hygiene, 101, 140-145.

Daş, G., Savaş, T., Kaufmann, F., Idris, A., Abel, H. \& Gauly, M. (2011) Precision, repeatability and representative ability of faecal egg counts in Heterakis gallinarum infected chickens. Veterinary Parasitology, 183, 87-94.

Daszak, P., Berger, L., Cunningham, A.A., Hyatt, A.D., Earl Green, D. \& Speare, R. (1999) Emerging infectious diseases and amphibian population declines. Emerging Infectious Diseases, 5, 735-748.

Daszak, P., Cunningham, A.A. \& Hyatt, A.D. (2000) Emerging infectious diseases of wildlife - Threats to biodiversity and human health. Science, 287, 443-449.

Davidson, R., Amundsen, H, Lie, N.O., Luyckx, K., Robertson, L.J., Verocai, G.G., Kutz, S. J. \& Ytrehus, B. (2014) Sentinels in a climatic outpost: Endoparasites in the introduced muskox (Ovibos moschatus wardi) population of Dovrefjell, Norway. International Journal for Parasitology: Parasites and Wildlife, 3, 154-160.

Davidson, R. K., Ličina, T., Gorini, L. \& Milner J. M. (2015) Endoparasites in a Norwegian moose (Alces alces) population - Faunal diversity, abundance and body condition. International Journal for Parasitology: Parasites and Wildlife 4, 29-36.

Dawkins, R. \& Krebs, J.R. (1979) Arms races between and within species. Proceedings of the Royal Society of London - Biological Sciences, 205, 489-511.

De Castro, F. \& Bolker, B. (2005) Mechanisms of disease-induced extinction. Ecology Letters, 8, 117-126.

De Roode, J.C., Pansini, R., Cheesman, S.J., Helinski, M.E.H., Huijben, S., Wargo, A.R., Bell, A.S., Chan, B.H.K., Walliker, D. \& Read, A.F. (2005) Virulence and competitive ability in genetically diverse malaria infections. Proceedings of the National Academy of Sciences of the United States of America, 102, 7624-7628.

Debeffe, L., McLoughlin, P.D., Medill, S.A., Stewart, K., Andres, D., Shury, T., Wagner, B., Jenkins, E., Gilleard, J.S. \& Poissant, J. (2016) Negative covariance between parasite load and body condition in a population of feral horses. Parasitology 143, 983-997.

Denwood, M.J., Love, S., Innocent, G.T., Matthews, L., McKendrick, I.J., Hillary, N., Smith, A. \& Reid, S.W.J. (2012) Quantifying the sources of variability in equine faecal egg counts: Implications for improving the utility of the method. Veterinary Parasitology, 188, $120-126$.

Di Minin, E., Laitila, J., Montesino-Pouzols, F., Leader-Williams, N., Slotow, R., Goodman, P.S., Conway, A.J. \& Moilanen, A. (2015) Identification of policies for a sustainable legal trade in rhinoceros horn based on population projection and socioeconomic models. Conservation Biology, 29, 545-555.

Diamond, J. (1986) Overview: Laboratory experiments, field experiments, and natural 
experiments. Community ecology (eds J. Diamond \& T. Case). Harper \& Row, New York.

Dick, C.W. \& Patterson, B.D. (2007) Against all odds: Explaining high host specificity in dispersal-prone parasites. International Journal for Parasitology, 37, 871-876.

Dieckmann, U., Metz, J.A.J., Sabelis, M.W. \& Sigmund, K. (2005) Adaptive Dynamics of Infectious Diseases: In Pursuit of Virulence Management. Cambridge University Press, Cambridge, UK.

Dobson, A., Lafferty, K.D., Kuris, A.M., Hechinger, R.F. \& Jetz, W. (2008) Homage to Linnaeus: How many parasites? How many hosts? Proceedings of the National Academy of Sciences of the United States of America, 105, 11482-11489.

Döpfer, D., Kerssens, C.M., Meijer, Y.G.M., Boersema, J.H. \& Eysker, M. (2004) Shedding consistency of strongyle-type eggs in dutch boarding horses. Veterinary Parasitology, 124, 249-258.

Duffy, M.A. \& Sivars-Becker, L. (2007) Rapid evolution and ecological host-parasite dynamics. Ecology Letters, 10, 44-53.

Dunn, R.R., Harris, N.C., Colwell, R.K., Koh, L.P. \& Sodhi, N.S. (2009) The sixth mass coextinction: Are most endangered species parasites and mutualists? Proceedings of the Royal Society B: Biological Sciences, 276, 3037-3045.

Dybdahl, M.F. \& Lively, C.M. (1998) Host-parasite coevolution: Evidence for rare advantage and time-lagged selection in a natural population. Evolution, 52, 1057-1066.

Dytham, C. (2003) Choosing and using statistics: a biologist's guide - 2nd ed. Blackwell Science, Oxford.

Eberhard, M.L., Kovacs-Nace, E., Blotkamp, J., Verwij, J.J., Asigri, V.A.A. \& Polderman, A.M. (2001) Experimental Oesophagostomum bifurcum in monkeys. Journal of Helminthology, 75, 51-56.

Emslie, R. (2011) Diceros bicornis ssp. longipes. The IUCN Red List of Threatened Species 2011. Downloaded on 17 September 2015.

Emslie, R. (2012a) Diceros bicornis ssp. minor. In: IUCN 2013. IUCN Red List of Threatened Species. Version 2013.1. Downloaded on 29 August 2013.

Emslie, R. (2012b) Diceros bicornis. In: IUCN 2013. IUCN Red List of Threatened Species. Version 2013.1. Downloaded on 29 August 2013.

Estes, R.D. (1991) The behavior guide to African mammals: including hoofed mammals, carnivores, primates. University of California Press.

Evenhuis, N.L. (2012) Sir Richard Owen's fly, Gyrostigma rhinocerontis (Diptera: Oestridae): correction of the authorship and date, with a list of animal names newly proposed by Owen in his little-known 1830 catalogue. Zootaxa, 3501, 74-82.

Ezenwa, V.O. (2003) Habitat overlap and gastrointestinal parasitism in sympatric African bovids. Parasitology, 126, 379-388.

Ezenwa, V.O., Etienne, R.S., Luikart, G., Beja-Pereira, A. \& Jolles, A.E. (2010) Hidden consequences of living in a wormy world: Nematode-induced immune suppression facilitates tuberculosis invasion in African buffalo. American Naturalist, 176, 613-624.

Ezenwa, V.O. \& Jolles, A.E. (2008) Horns honestly advertise parasite infection in male and female African buffalo. Animal Behaviour, 75, 2013-2021. 
Faith, D.P., Reid, C.A.M. \& Hunter, J. (2004) Integrating Phylogenetic Diversity, Complementarity, and Endemism for Conservation Assessment. Conservation Biology, 18, 255-261.

Fenton, A. \& Rands, S.A. (2004) Optimal parasite infection strategies: A state-dependent approach. International Journal for Parasitology, 34, 813-821.

Ferreira, S.M., Greaver, C.C. \& Knight, M.H. (2011) Assessing the population performance of the black rhinoceros in Kruger National Park. South African Journal of Wildlife Research, 41, 192-204.

Ferreira, S.M., Pfab, M. \& Knight, M. (2014) Management strategies to curb rhino poaching: Alternative options using a cost-benefit approach. South African Journal of Science, 110.

Fisher, M.C., Garner, T.W.J. \& Walker, S.F. (2009) Global emergence of Batrachochytrium dendrobatidis and amphibian chytridiomycosis in space, time, and host. Annual Review of Microbiology, pp. 291-310.

Forbes, A.B., Huckle, C.A., Gibb, M.J., Rook, A.J. \& Nuthall, R. (2000) Evaluation of the effects of nematode parasitism on grazing behaviour, herbage intake and growth in young grazing cattle. Veterinary Parasitology, 90, 111-118.

Forbes, M.R.L. (1993) Parasitism and host reproductive effort. Oikos, 67, 444-450.

Frank, S.A. (1992) A kin selection model for the evolution of virulence. Proceedings of the Royal Society B: Biological Sciences, 250, 195-197.

Frank, S.A. (1996) Models of parasite virulence. Quarterly Review of Biology, 71, 37-78.

Freeman, E.W., Meyer, J.M., Adendorff, J., Schulte, B.A. \& Santymire, R.M. (2014) Scraping behavior of black rhinoceros is related to age and fecal gonadal metabolite concentrations. Journal of Mammalogy, 95, 340-348.

Frick, W.F., Pollock, J.F., Hicks, A.C., Langwig, K.E., Reynolds, D.S., Turner, G.G., Butchkoski, C.M. \& Kunz, T.H. (2010) An emerging disease causes regional population collapse of a common North American bat species. Science, 329, 679-682.

Fuentes, M.V., Sainz-Elipe, S. \& Galán-Puchades, M.T. (2007) Ecological study of the wood mouse helminth community in a burned Mediterranean ecosystem in regeneration five years after a wildfire. Acta Parasitologica, 52, 403-413.

Fyumagwa, R.D., Runyoro, V., Horak, I.G. \& Hoare, R. (2007) Ecology and control of ticks as disease vectors in wildlife of the Ngorongoro Crater, Tanzania. South African Journal of Wildlife Research, 37, 79-90.

Galvani, A.P. (2003) Immunity, antigenic heterogeneity, and aggregation of helminth parasites. Journal of Parasitology, 89, 232-241.

Gandon, S., Mackinnon, M., Nee, S. \& Read, A. (2003) Imperfect vaccination: Some epidemiological and evolutionary consequences. Proceedings of the Royal Society B: Biological Sciences, 270, 1129-1136.

Gasbarre, L.C., Leighton, E.A. \& Bryant, D. (1996) Reliability of a single fecal egg per gram determination as a measure of individual and herd values for trichostrongyle nematodes of cattle. American Journal of Veterinary Research, 57, 168-171.

Gates, C.C., Elkin, B.T. \& Dragon, D.C. (1995) Investigation, control and epizootiology of anthrax in a geographically isolated, free-roaming bison population in northern Canada. 
Canadian journal of veterinary research $=$ Revue canadienne de recherche vétérinaire, 59, 256-264.

George-Nascimento, M., Muñoz, G., Marquet, P.A. \& Poulin, R. (2004) Testing the energetic equivalence rule with helminth endoparasites of vertebrates. Ecology Letters, 7, 527531.

Gethings, O. J., Sage, R. B. \& Leather, S. R. (2016) Density-dependent regulation of fecundity in Syngamus trachea infrapopulations in semi-naturally occurring ring-necked pheasants (Phasianus colchicus) and wild Carrion Crows (Corvus corone). Parasitology, 143, 716-722.

Gillespie, T.R. (2006) Noninvasive assessment of gastrointestinal parasite infections in freeranging primates. International Journal of Primatology, 27, 1129-1143.

Goddard, J. (1968) Food preference of two black rhinoceros populations. East African Wildlife Journal 6, 1-18.

Gómez, A. \& Nichols, E. (2013) Neglected wild life: Parasitic biodiversity as a conservation target. International Journal for Parasitology: Parasites and Wildlife, 2, 222-227.

Gooderham, K. \& Schulte-Hostedde, A. (2011) Macroparasitism influences reproductive success in red squirrels (Tamiasciurus hudsonicus). Behavioral Ecology, 22, 11951200.

Goossens, B., Osaer, S. \& Kora, S. (1997) Long-term effects of an experimental infection with Trypanosoma congolense on reproductive performance of trypanotolerant Djallonke ewes and West African Dwarf does. Research in Veterinary Science, 63, 169-173.

Gortázar, C., Acevedo, P., Ruiz-Fons, F. \& Vicente, J. (2006) Disease risks and overabundance of game species. European Journal of Wildlife Research, 52, 81-87.

Gregory, R.D. \& Woolhouse, M.E.J. (1993) Quantification of parasite aggregation: A simulation study. Acta Tropica, 54, 131-139.

Grenfell, B.T. \& Dobson, A.P. (1995) Ecology of Infectious Diseases in Natural Populations. Cambridge University Press Cambridge, UK.

Grobler, D.G., Raath, J.P., Braack, L.E., Keet, D.F., Gerdes, G.H., Barnard, B.J., Kriek, N.P., Jardine, J. \& Swanepoel, R. (1995) An outbreak of encephalomyocarditis-virus infection in free-ranging African elephants in the Kruger National Park. The Onderstepoort journal of veterinary research, 62, 97-108.

Gross, S. J., Ryan, W.G. and Ploeger, H.W. (1999) Anthelmintic treatment of adult dairy cows and the effect on milk production. Veterinary Record 144, 581-587.

Gulland, F.M.D. (1992) The role of nematode parasites in Soay sheep (Ovis aries L.) mortality during a population crash. Parasitology, 105, 493-503.

Gunn, A. \& Irvine, R.J. (2003) Subclinical parasitism and ruminant foraging strategies - A review. Wildlife Society Bulletin, 31, 117-126.

Gurnell, J., Rushton, S.P., Lurz, P.W.W., Sainsbury, A.W., Nettleton, P., Shirley, M.D.F., Bruemmer, C. \& Geddes, N. (2006) Squirrel poxvirus: Landscape scale strategies for managing disease threat. Biological Conservation, 131, 287-295.

Hamilton, W.D. \& Zuk, M. (1982) Heritable true fitness and bright birds: A role for parasites? Science, 218, 384-387.

Harris, N.C., Livieri, T.M. \& Dunn, R.R. (2014) Ectoparasites in Black-footed Ferrets (Mustela 
nigripes) from the Largest Reintroduced Population of the Conata Basin, South Dakota, USA. Journal of Wildlife Diseases, 50, 340-3.

Hastie, L.C. \& Young, M.R. (2001) Freshwater pearl mussel (Margaritifera margaritifera) glochidiosis in wild and farmed salmonid stocks in Scotland. Hydrobiologia, 445, 109119.

Haukisalmi, V. \& Henttonen, H. (1990) The impact of climatic factors and host density on the long-term population dynamics of vole helminths. Oecologia, 83, 309-315.

Haydon, D.T., Randall, D.A., Matthews, L., Knobel, D.L., Tallents, L.A., Gravenor, M.B., Williams, S.D., Pollinger, J.P., Cleaveland, S., Woolhouse, M.E.J., Sillero-Zubiri, C., Marino, J., Macdonald, D.W. \& Laurenson, M.K. (2006) Low-coverage vaccination strategies for the conservation of endangered species. Nature, 443, 692-695.

Hayward, A.D. (2013) Causes and consequences of intra- and inter-host heterogeneity in defence against nematodes. Parasite Immunology, 35, 362-373.

Heard, M.J., Smith, K.F., Ripp, K.J., Berger, M., Chen, J., Dittmeier, J., Goter, M., McGarvey, S.T. \& Ryan, E. (2013) The Threat of Disease Increases as Species Move Toward Extinction. Conservation Biology.

Hieronymus, T., Witmer, L.M. \& Ridgely, R.C. (2006) Structure of white rhinoceros (Ceratotherium simum) horn investigated by X-ray computed tomography and histology with implications for growth and external form. Journal of Morphology, 267, 1172-1176.

Higes, M., Martín, R. \& Meana, A. (2006) Nosema ceranae, a new microsporidian parasite in honeybees in Europe. Journal of Invertebrate Pathology, 92, 93-95.

Hillegass, M.A., Waterman, J.M. \& Roth, J.D. (2010) Parasite removal increases reproductive success in a social African ground squirrel. Behavioral Ecology, 21, 696-700.

Hines, A.M., Ezenwa, V.O., Cross, P. \& Rogerson, J.D. (2007) Effects of supplemental feeding on gastrointestinal parasite infection in elk (Cervus elaphus): Preliminary observations. Veterinary Parasitology, 148, 350-355.

Hitchins, P.M. (1984) Translocation of Black Rhinoceros (Diceros bicornis minor) from the Natal Game Reserves 1962-1983. Lammergeyer, 33, 45-48.

Hitchins, P.M. \& Keep, M.E. (1970) Observations on skin lesions of the black rhinoceros (Diceros bicornis Linn.) in the Hluhluwe Game Reserve, Zululand. Lammergeyer, 12, 56-65, pls. 51-56, figs. 51-52, table 51.

Holland, C.V. (2009) Predisposition to ascariasis: Patterns, mechanisms and implications. Parasitology, 136, 1537-1547.

Hudson, P.J. \& Dobson, A.P. (1995) Macroparasites: Observed Patterns in Naturally Fluctuating Animal Populations. Ecology of Infectious Diseases in Natural Populations (eds B.T. Grenfell \& A.P. Dobson). Cambridge University Press, Cambridge.

Hudson, P.J., Dobson, A.P. \& Lafferty, K.D. (2006) Is a healthy ecosystem one that is rich in parasites? Trends in Ecology and Evolution, 21, 381-385.

Hudson, P.J., Dobson, A.P. \& Newborn, D. (1998) Prevention of population cycles by parasite removal. Science, 282, 2256-2258.

Hudson, P.J., Newborn, D. \& Dobson, A.P. (1992) Regulation and stability of a free-living hostparasite system: Trichostrongylus tenuis in red grouse. I. Monitoring and parasite 
reduction experiments. Journal of Animal Ecology, 61, 477-486.

Hughes, J., Albon, S.D., Irvine, R.J. \& Woodin, S. (2009) Is there a cost of parasites to caribou? Parasitology, 136, 253-265.

Hughes, S. \& Kelly, P. (2006) Interactions of malnutrition and immune impairment, with specific reference to immunity against parasites. Parasite Immunology, 28, 577-588.

Hunter, P., Swanepoel, S.P., Esterhuysen, J.J., Raath, J.P., Bengis, R.G. \& Van der Lugt, J.J. (1998) The efficacy of an experimental oil-adjuvanted encephalomyocarditis vaccine in elephants, mice and pigs. Vaccine, 16, 55-61.

Hyslop St, N.G. (1979) Observations on the survival and infectivity of airborne rinderpest virus. International Journal of Biometeorology, 23, 1-7.

IBM (2011) SPSS Statistics Version 20.

Irvine, R.J. (2006) Parasites and the dynamics of wild mammal populations. Animal Science, 82, 775-781.

IUCN (2013) The IUCN Red List of Threatened Species. Version 2012.2. http://www.iucnredlist.org. Downloaded on 30 April 2013.

Jagla, E., Spiewak, J., Zalesny, G. \& Popiolek, M. (2013) Effect of storage and preservation of horse faecal samples on the detectability and viability of strongylid nematode eggs and larvae. Bulletin of the Veterinary Institute in Pulawy, 57, 161-165.

Jansen, P.A., Kristoffersen, A.B., Viljugrein, H., Jimenez, D., Aldrin, M. \& Stien, A. (2012) Sea lice as a density-dependent constraint to salmonid farming. Proceedings. Biological sciences / The Royal Society, 279, 2330-2338.

Johnson, P.T.J. \& Hoverman, J.T. (2014) Heterogeneous hosts: How variation in host size, behaviour and immunity affects parasite aggregation. Journal of Animal Ecology, 83, 1103-1112.

Johnson, S. \& Gerding, D.N. (1998) Clostridium difficile - Associated diarrhea. Clinical Infectious Diseases, 26, 1027-1036.

Jørgensen, D. (2014) Conservation implications of parasite co-reintroduction. Conservation Biology.

Kaufmann, J. (1996) Parasitic Infections of Domestic Animals: A Diagnostic Manual. Birkhäuser, Switzerland.

Keep, M.E. (1970) A checklist of the blood parasites recorded from the larger wild mammals in Zululand. Lammergeyer, 11, 54-57.

Kerley, G.I.H., Knight, M.H. \& De Kock, M. (1995) Desertification of subtropical thicket in the Eastern Cape, South Africa: Are there alternatives? Environmental Monitoring and Assessment, 37, 211-230.

Kerr, J.T., Kharouba, H.M. \& Currie, D.J. (2007) The macroecological contribution to global change solutions. Science, 316, 1581-1584.

Keusch, G.T. \& Migasena, P. (1982) Biological implications of polyparasitism. Reviews of Infectious Diseases, 4, 880-882.

King, J.M. (1969) The Capture and Translocation of the Black Rhinoceros. East African Wildlife Journal, 7, 115-130.

Kjær, L.N., Lungholt, M.M., Nielsen, M.K., Olsen, S.N. \& Maddox-Hyttel, C. (2007) Interpretation of serum antibody response to Anoplocephala perfoliata in relation to 
parasite burden and faecal egg count. Equine Veterinary Journal, 39, 529-533.

Klingel, H. \& Klingel, U. (1966) The rhinoceroses of Ngorongoro Crater. ORYX, 8, 302-306. Knapp, S.E., Krecek, R.C., Horak, I.G. \& Penzhorn, B.L. (1997) Helminths and arthropods of black and white rhinoceroses in Southern Africa. Journal of Wildlife Diseases, 33, 492502.

Knobel, D.L., Fooks, A.R., Brookes, S.M., Randall, D.A., Williams, S.D., Argaw, K., Shiferaw, F., Tallents, L.A. \& Laurenson, M.K. (2008) Trapping and vaccination of endangered Ethiopian wolves to control an outbreak of rabies. Journal of Applied Ecology, 45, 109116.

Kock, N.D. \& Kock, M.D. (1990) Skin lesions in free-ranging black rhinoceroses (Diceros bicornis) in Zimbabwe. Journal of Zoo Animal Medicine, 21, 447-452, figs. 441-446.

Kock, R.A., Wambua, J.M., Mwanzia, J., Wamwayi, H., Ndungu, E.K., Barrett, T., Kock, N.D. \& Rossiter, P.B. (1999) Rinderpest epidemic in wild ruminants in Kenya 1993-97. Veterinary Record, 145, 275-283.

Koh, L.P., Dunn, R.D., Sodhi, N.S., Colwell, R.K., Proctor, H.C. \& Smith, V.S. (2004) Species co-extinctions and the biodiversity crisis. Science, 305, 1632-1634.

Krauth, S.J., Coulibaly, J.T., Knopp, S., Traoré, M., N'Goran, E.K. \& Utzinger, J. (2012) An In-Depth Analysis of a Piece of Shit: Distribution of Schistosoma mansoni and Hookworm Eggs in Human Stool. PLoS Neglected Tropical Diseases, 6, e1969.

Krebs, J.R. \& Davies, N.B. (1993) An Introduction to Behavioural Ecology, 3rd Edition edn. Blackwell Science Ltd.

Kuris, A.M., Hechinger, R.F., Shaw, J.C., Whitney, K.L., Aguirre-Macedo, L., Boch, C.A., Dobson, A.P., Dunham, E.J., Fredensborg, B.L., Huspeni, T.C., Lorda, J., Mababa, L., Mancini, F.T., Mora, A.B., Pickering, M., Talhouk, N.L., Torchin, M.E. \& Lafferty, K.D. (2008) Ecosystem energetic implications of parasite and free-living biomass in three estuaries. Nature, 454, 515-518.

Kuzmina, T.A., Lyons, E.T., Tolliver, S.C., Dzeverin, I.I. \& Kharchenko, V.A. (2012) Fecundity of various species of strongylids (Nematoda: Strongylidae)- parasites of domestic horses. Parasitology Research, 111, 2265-2271.

Labocha, M.K., Schutz, H. \& Hayes, J.P. (2014) Which body condition index is best? Oikos, 123, 111-119.

Lafferty, K.D. (2004) Fishing for lobsters indirectly increases epidemics in sea urchins. Ecological Applications, 14, 1566-1573.

Lafferty, K.D. \& Gerber, L.R. (2002) Good medicine for conservation biology: The intersection of epidemiology and conservation theory. Conservation Biology, 16, 593-604.

Lagrot, I., Lagrot, J.F. \& Bour, P. (2007) Probable extinction of the western black rhino, Diceros bicornis longipes: 2006 survey in northern Cameroon. Pachyderm, 43, 19-28.

Landman, M., Schoeman, D.S. \& Kerley, G.I.H. (2013) Shift in Black Rhinoceros Diet in the Presence of Elephant: Evidence for Competition? PLoS ONE, 8, e69771.

Lange, B., Reuter, M., Ebert, D., Muylaert, K. \& Decaestecker, E. (2014) Diet quality determines interspecific parasite interactions in host populations. Ecology and Evolution, 4, 3093-3102.

Laubscher, L. \& Hoffman, L. (2012) An overview of disease-free buffalo breeding projects 
with reference to the different systems used in South Africa. Sustainability, 4, 31243140.

Law, P.R., Fike, B. \& Lent, P.C. (2013) Mortality and female fecundity in an expanding black rhinoceros (Diceros bicornis minor) population. European Journal of Wildlife Research, 59, 477-485.

Law, P.R. \& Linklater, W.L. (2014) Black rhinoceros demography should be stage, not age, based. African Journal of Ecology, 52, 571-573.

Lebarbenchon, C., Poulin, R., Gauthier-Clerc, M. \& Thomas, F. (2006) Parasitological consequences of overcrowding in protected areas. EcoHealth, 3, 303-307.

Leggett, H.C., Brown, S.P. \& Reece, S.E. (2014) War and peace: Social interactions in infections. Philosophical Transactions of the Royal Society B: Biological Sciences, 369.

Leggett, H.C., Buckling, A., Long, G.H. \& Boots, M. (2013) Generalism and the evolution of parasite virulence. Trends in Ecology and Evolution, 28, 592-596.

Lello, J., Boag, B., Fenton, A., Stevenson, I.R. \& Hudson, P.J. (2004) Competition and mutualism among the gut helminths of a mammalian host. Nature, 428, 840-844.

Lichtenfels, J.R., Kharchenko, V.A. \& Dvojnos, G.M. (2008) Illustrated identification keys to strongylid parasites (strongylidae: Nematoda) of horses, zebras and asses (Equidae). Veterinary Parasitology, 156, 4-161.

Linklater, W.L., Adcock, K., du Preez, P., Swaisgood, R.R., Law, P.R., Knight, M.H., Gedir, J.V. \& Kerley, G.I. (2011) Guidelines for large herbivore translocation simplified: Black rhinoceros case study. Journal of Applied Ecology, 48, 493-502.

Linklater, W.L., Gedir, J.V., Law, P.R., Swaisgood, R.R., Adcock, K., Preez, P., Knight, M.H. \& Kerley, G.I.H. (2012) Translocations as experiments in the ecological resilience of an asocial mega-herbivore. PLOS ONE, 7, e30664.

Linklater, W.L., MacDonald, E.A., Flamand, J.R.B. \& Czekala, N.M. (2010) Declining and low fecal corticoids are associated with distress, not acclimation to stress, during the translocation of African rhinoceros. Animal Conservation, 13, 104-111.

Lips, K.R., Brem, F., Brenes, R., Reeve, J.D., Alford, R.A., Voyles, J., Carey, C., Livo, L., Pessier, A.P. \& Collins, J.P. (2006) Emerging infectious disease and the loss of biodiversity in a Neotropical amphibian community. Proceedings of the National Academy of Sciences of the United States of America, 103, 3165-3170.

Lipsitch, M., Siller, S. \& Nowak, M.A. (1996) The evolution of virulence in pathogens with vertical and horizontal transmission. Evolution, 50, 1729-1741.

Little, T.J., Shuker, D.M., Colegrave, N., Day, T. \& Graham, A.L. (2010) The coevolution of virulence: Tolerance in perspective. PLoS Pathogens, 6, e1001006.

Lively, C.M. \& Dybdahl, M.F. (2000) Parasite adaptation to locally common host genotypes. Nature, 405, 679-681.

Lloyd-Smith, J.O., Cross, P.C., Briggs, C.J., Daugherty, M., Getz, W.M., Latto, J., Sanchez, M.S., Smith, A.B. \& Swei, A. (2005) Should we expect population thresholds for wildlife disease? Trends in Ecology and Evolution, 20, 511-519.

Lloyd-Smith, J.O., Getz, W.M. \& Westerhoff, H.V. (2004) Frequency-dependent incidence in models of sexually transmitted diseases: Portrayal of pair-based transmission and effects of illness on contact behaviour. Proceedings of the Royal Society B: Biological 
Sciences, 271, 625-634.

Lochmiller, R.L. \& Deerenberg, C. (2000) Trade-offs in evolutionary immunology: Just what is the cost of immunity? Oikos, 88, 87-98.

Loker, E. \& Hofkin, B. (2015) Parasitology: A Conceptual Approach. Garland Science.

Loo, J.A. (2009) Ecological impacts of non-indigenous invasive fungi as forest pathogens. Biological Invasions, 11, 81-96.

López-Villavicencio, M., Courjol, F., Gibson, A.K., Hood, M.E., Jonot, O., Shykoff, J.A. \& Giraud, T. (2011) Competition, cooperation among kin, and virulence in multiple infections. Evolution, 65, 1357-1366.

López, G., López-Parra, M., Fernández, L., Martínez-Granados, C., Martínez, F., Meli, M.L., Gil-Sánchez, J.M., Viqueira, N., Díaz-Portero, M.A., Cadenas, R., Lutz, H., Vargas, A. \& Simón, M.A. (2009) Management measures to control a feline leukemia virus outbreak in the endangered Iberian lynx. Animal Conservation, 12, 173-182.

Luong, L.T., Vigliotti, B.A. \& Hudson, P.J. (2011) Strong density-dependent competition and acquired immunity constrain parasite establishment: Implications for parasite aggregation. International Journal for Parasitology, 41, 505-511.

Lynsdale, C.L., dos Santos, D.J.F., Hayward, A.D., Mar, K.U., Htut, W., Aung, H.H., Soe, A.T. \& Lummaa, V. (2015) A standardised faecal collection protocol for intestinal helminth egg counts in Asian elephants, Elephas maximus. International Journal for Parasitology: Parasites and Wildlife, 4, 307-315.

Macdonald, D. (2006) The Encyclopedia of Mammals. Oxford University Press, Oxford, UK.

MacPhee, R.D.E. \& Sues, H.-D. (1999) Extinctions in Near Time: Causes, Contexts, and Consequences. Springer, Berlin.

Mage, C. and Dorchies, P. (1998) Paramphistomose des bovins: etude des relations coproscopie-populations parasitaires. Revue De Medecine Veterinaire 149, 927-929.

Manga-González, M.Y., González-Lanza, C., Cabanas, E. \& Campo, R. (2001) Contributions to and review of dicrocoeliosis, with special reference to the intermediate hosts of Dicrocoelium dendriticum. Parasitology, 123, S91-S114.

Martínez-Padilla, J., Redpath, S.M., Zeineddine, M. \& Mougeot, F. (2014) Insights into population ecology from long-term studies of red grouse Lagopus lagopus scoticus. Journal of Animal Ecology, 83, 85-98.

May, R.M. \& Anderson, R.M. (1979) Population biology of infectious diseases: II. Nature, 280, 455-461.

May, R.M. \& Anderson, R.M. (1987) Transmission dynamics of HIV infection. Nature, 326, 137-142.

Mazoir, N., Benharref, A., Bailén, M., Reina, M., González-Coloma, A. \& Martínez-Díaz, R.A. (2011) Antileishmanial and antitrypanosomal activity of triterpene derivatives from latex of two euphorbia species. Zeitschrift fur Naturforschung - Section C Journal of Biosciences, 66 C, 360-366.

McCallum, H., Barlow, N. \& Hone, J. (2001) How should pathogen transmission be modelled? Trends in Ecology and Evolution, 16, 295-300.

McCallum, H.I. (1990) Covariance in parasite burdens: The effect of predisposition to infection. Parasitology, 100, 153-159. 
McCraw, B.M. \& Slocombe, J.O. (1976) Strongylus vulgaris in the horse: a review. Canadian Veterinary Journal, 17, 150-157.

McCurdy, D.G., Shutler, D., Mullie, A. \& Forbes, M.R. (1998) Sex-biased parasitism of avian hosts: Relations to blood parasite taxon and mating system. Oikos, 82, 303-312.

McGill, I., Feltrer, Y., Jeffs, C., Sayers, G., Marshall, R.M., Peirce, M.A., Stidworthy, M.P., Pocknell, A.M. \& Sainsbury, A.W. (2010) Isosporoid coccidiosis in translocated cirl buntings (Emberiza cirlus). Veterinary Record, 167, 656-660.

McKenna, P.B. (1981) The diagnostic value and interpretation of faecal egg counts in sheep. New Zealand Veterinary Journal, 29, 129-132.

McMahon, T.A., Brannelly, L.A., Chatfield, M.W.H., Johnson, P.T.J., Joseph, M.B., McKenzie, V.J., Richards-Zawacki, C.L., Venesky, M.D. \& Rohr, J.R. (2013) Chytrid fungus Batrachochytrium dendrobatidis has nonamphibian hosts and releases chemicals that cause pathology in the absence of infection. Proceedings of the National Academy of Sciences of the United States of America, 110, 210-215.

Meana, A., Luzon, M., Corchero, J. \& Gómez-Bautista, M. (1998) Reliability of coprological diagnosis of Anoplocephala perfoliata infection. Veterinary Parasitology 74, 79-83.

Méthot, P.O. (2012) Why do parasites harm their host? on the origin and legacy of theobald Smith's "law of Declining Virulence" - 1900-1980. History and Philosophy of the Life Sciences, 34, 561-601.

Michalakis, Y., Olivieri, I., Renaud, F. \& Raymond, M. (1992) Pleiotropic action of parasites: How to be good for the host. Trends in Ecology and Evolution, 7, 59-62.

Michel, J. F. (1968) Faecal egg counts in infections of gastrointestinal nematoes in cows. Veterinary Record 82, 132-3.

Mihok, S., Kock, R. \& Masake, R. (1995) Health implications of translocations of endangered species in Africa: trypanosomiasis in rhinoceros. Proceedings of the Joint Conference of American Association of Zoo Veterinarians, Wildlife Disease Association and American Association of Wildl. Vets, August.

Mihok, S., Munyoki, E., Brett, R.A., Jonyo, J.F., Rottcher, D., Majiwa, P.A.O., Kang'ethe, E.K., Kaburia, H.F.A. \& Zweygarth, E. (1992) Trypanosomiasis and the conservation of black rhinoceros (Diceros bicornis) at the Ngulia Rhino Sanctuary, Tsavo West National Park, Kenya. African Journal of Ecology, 30, 103-115.

Miller, M.W., Vayhinger, J.E., Bowden, D.C., Roush, S.P., Verry, T.E., Torres, A.N. \& Jurgens, V.D. (2000) Drug treatment for lungworm in bighorn sheep: Reevaluation of a 20-yearold management prescription. Journal of Wildlife Management, 64, 505-512.

Miura, O., Kuris, A.M., Torchin, M.E., Hechinger, R.F., Dunham, E.J. \& Chiba, S. (2005) Molecular-genetic analyses reveal cryptic species of trematodes in the intertidal gastropod, Batillaria cumingi (Crosse). International Journal for Parasitology, 35, 793-801.

Mock, M. \& Fouet, A. (2001) Anthrax. Annual Review of Microbiology, pp. 647-671.

Moir, M.L., Vesk, P.A., Brennan, K.E.C., Poulin, R., Hughes, L., Keith, D.A., McCarthy, M.A. \& Coates, D.J. (2012) Considering Extinction of Dependent Species during Translocation, Ex Situ Conservation, and Assisted Migration of Threatened Hosts. Conservation Biology, 26, 199-207. 
Morand, S. (1996) Life-history traits in parasitic nematodes: A comparative approach for the search of invariants. Functional Ecology, 10, 210-218.

Morrill, A. \& Forbes, M.R. (2012) Random parasite encounters coupled with condition-linked immunity of hosts generate parasite aggregation. International Journal for Parasitology, 42, 701-706.

Mouritsen, K.N. \& Poulin, R. (2002) Parasitism, climate oscillations and the structure of natural communities. Oikos, 97, 462-468.

Mukhopadhyay, A.K., Taylor, W.P. \& Roeder, P.L. (1999) Rinderpest: A case study of animal health emergency management. OIE Revue Scientifique et Technique, 18, 164-178.

Murray, D.L., Cary, J.R. \& Keith, L.B. (1997) Interactive effects of sublethal nematodes and nutritional status on snowshoe hare vulnerability to predation. Journal of Animal Ecology, 66, 250-264.

Nalubamba, K.S., Mudenda, N.B. \& Malamo, M.R. (2012) A seasonal survey of gastrointestinal parasites in captive wild impala antelope on a game facility south of Lusaka, Zambia. Journal of Helminthology, 86, 418-425.

Narsapur, V.S. (1988). Pathogenesis and biology of anoplocephaline cestodes of domestic animals. Annales de Recherches Veterinaires, 19, 1-17.

Nielsen, M.K. (2012) Sustainable equine parasite control: Perspectives and research needs. Veterinary Parasitology, 185, 32-44.

Nielsen, M. K. (2016) Equine tapeworm infections: Disease, diagnosis and control. Equine Veterinary Education 28, 388-395.

Nielsen, M.K., Baptiste, K.E., Tolliver, S.C., Collins, S.S. \& Lyons, E.T. (2010a) Analysis of multiyear studies in horses in Kentucky to ascertain whether counts of eggs and larvae per gram of feces are reliable indicators of numbers of strongyles and ascarids present. Veterinary Parasitology, 174, 77-84.

Nielsen, M.K., Haaning, N. \& Olsen, S.N. (2006) Strongyle egg shedding consistency in horses on farms using selective therapy in Denmark. Veterinary Parasitology, 135, 333-335.

Nielsen, M.K., Vidyashankar, A.N., Andersen, U.V., DeLisi, K., Pilegaard, K. \& Kaplan, R.M. (2010b) Effects of fecal collection and storage factors on strongylid egg counts in horses. Veterinary Parasitology, 167, 55-61.

Nijhof, A.M., Penzhorn, B.L., Lynen, G., Mollel, J.O., Morkel, P., Bekker, C.P.J. \& Jongejan, F. (2003) Babesia bicornis sp. nov. and Theileria bicornis sp. nov.: Tick-borne parasites associated with mortality in the black rhinoceros (Diceros bicornis). Journal of Clinical Microbiology, 41, 2249-2254.

Nilsson, O., B.-L. Ljungstöm, J. Höglund, H. Lundquist and A. Uggla (1995). "Anoplocephala perfoliata in horses in Sweden: Prevalence, infection levels and intestinal lesions." Acta Veterinaria Scandinavica 36: 319-328.

Noble, E.R. \& Noble, G.A. (1982) Parasitology: The Biology of Animal Parasites. Lea \& Febiger, Philadelphia, U.S.A.

Nordling, D., Andersson, M., Zohari, S. \& Gustafsson, L. (1998) Reproductive effort reduces specific immune response and parasite resistance. Proceedings of the Royal Society B: Biological Sciences, 265, 1291-1298.

Normile, D. (2008) Driven to extinction. Science, 319, 1606-1609. 
Norval, R.A.I., Andrew, H.R. \& Yunker, C.E. (1989) Pheromone-mediation of host-selection in bont ticks (Amblyomma hebraeum Koch). Science, 243, 364-365.

Norval, R.A.I., Sutherst, R.W., Kurki, J., Gibson, J.D. \& Kerr, J.D. (1988) The effect of the brown ear-tick Rhipicephalus appendiculatus on the growth of Sanga and European Breed cattle. Veterinary Parasitology, 30, 149-164.

Nunn, C.L., Altizer, S., Sechrest, W., Jones, K.E., Barton, R.A. \& Gittleman, J.L. (2004) Parasites and the evolutionary diversification of primate clades. American Naturalist, 164.

Nunn, C.L., Brezine, C., Jolles, A.E. \& Ezenwa, V.O. (2014) Interactions between micro- and macroparasites predict microparasite species richness across primates. American Naturalist, 183, 494-505.

Ocaido, M., Siefert, L. \& Baranga, J. (1999) Seasonal changes of impala (Aepyceros melampus, Lichtenstein, 1812) faecal helminth egg counts through a one-year period. African Journal of Ecology, 37, 327-333.

OED (2015a) "disease, $n . "$. Oxford University Press.

OED (2015b) "parasite, $n . "$. Oxford University Press.

OED (2015c) "virulence, $n . "$. Oxford University Press.

Onah, D. N. \& Nawa, Y. (2000) Mucosal immunity against parasitic gastrointestinal nematodes. Korean Journal of Parasitology, 38, 209-236.

Owen-Smith, R.N. (1988) Megaherbivores: The influence of very large body size on ecology. Cambridge University Press, Cambridge.

Packer, C., Holt, R.D., Hudson, P.J., Lafferty, K.D. \& Dobson, A.P. (2003) Keeping the herds healthy and alert: Implications of predator control for infectious disease. Ecology Letters, 6, 797-802.

Pal, C., Maciá, M.D., Oliver, A., Schachar, I. \& Buckling, A. (2007) Coevolution with viruses drives the evolution of bacterial mutation rates. Nature, 450, 1079-1081.

Paterson, S., Wilson, K. \& Pemberton, J.M. (1998) Major histocompatibility complex variation associated with juvenile survival and parasite resistance in a large unmanaged ungulate population (Ovis aries L.). Proceedings of the National Academy of Sciences of the United States of America, 95, 3714-3719.

Pavone, S., Veronesi, F., Piergili Fioretti, D. \& Mandara, M.T. (2010) Pathological changes caused by Anoplocephala perfoliata in the equine ileocecal junction. Veterinary Research Communications, 34, S53-S56.

Pedersen, A.B. \& Fenton, A. (2007) Emphasizing the ecology in parasite community ecology. Trends in Ecology and Evolution, 22, 133-139.

Pedersen, A.B., Jones, K.E., Nunn, C.L. \& Altizer, S. (2007) Infectious diseases and extinction risk in wild mammals. Conservation Biology, 21, 1269-1279.

Peig, J. \& Green, A.J. (2009) New perspectives for estimating body condition from mass/length data: The scaled mass index as an alternative method. Oikos, 118, 1883-1891.

Penzhorn, B.L., Krecek, R.C., Horak, I.G., Verster, A.J.M., Walker, J.B., Boomker, J.D.F., Knapp, S.E. \& Quandt, S.K.F. (1994) Parasites of African rhinos: a documentation. pp. 168-175, tables 1-2. In: Penzhorn, B.L. et al. 1994 Proceedings of a symposium on rhinos as game ranch animals. Onderstepoort, Republic of South Africa, 9-10 
September 1994, pp. $i$-iv, 1-242.

Perrin N. \& Sibly, R.M. (1993) Dynamic models of energy allocation and investment. Annual Reviews of Ecology and Systematics, 24, 379-410.

Pienaar, D.J., Hall-Marin, A.J. \& Hitchins, P.M. (1991) Horn growth rates of free-ranging white and black rhinoceros. Koedoe, 34, 97-105.

Ploeger, H.W., Kloosterman, A., Rietveld, F.W., Berghen, P., Hilderson, H. \& Hollanders, W. (1994) Quantitative estimation of the level of exposure to gastrointestinal nematode infection in first-year calves. Veterinary Parasitology, 55, 287-315.

Plotz, R.D. (2014) The interspecific relationships of black rhinoceros (Diceros bicornis) in Hluhluwe-iMfolozi Park. PhD Thesis, Victoria University of Wellington.

Plotz, R.D. \& Linklater, W.J. (2009) Black rhinoceros (Diceros bicornis) calf succumbs after lion predation attempt: implications for conservation management. African Zoology, 44, 283-287.

Pollitt, L.C., Colegrave, N., Khan, S.M., Sajid, M. \& Reece, S.E. (2010) Investigating the evolution of apoptosis in malaria parasites: The importance of ecology. Parasites and Vectors, 3.

Potter, I.C. \& Hilliard, R.W. (2009) A proposal for the functional and phylogenetic significance of differences in the dentition of lampreys (Agnatha: Petromyzontiformes). Journal of Zoology, 212, 713-737.

Poulin, R. (1996) Sexual inequalities in helminth infections: A cost of being a male? American Naturalist, 147, 287-295.

Poulin, R. (1997) Population abundance and sex ratio in dioecious helminth parasites. Oecologia, 111, 375-380.

Poulin, R. (2000) Manipulation of host behaviour by parasites: A weakening paradigm? Proceedings of the Royal Society B: Biological Sciences, 267, 787-792.

Poulin, R. (2007a) Are there general laws in parasite ecology? Parasitology, 134, 763-776.

Poulin, R. (2007b) Evolutionary Ecology of Parasites, 2nd Edition edn. Princeton University Press, Princeton \& Oxford.

Poulin, R. \& George-Nascimento, M. (2007) The scaling of total parasite biomass with host body mass. International Journal for Parasitology, 37, 359-364.

Pourrut, X., Délicat, A., Rollin, P.E., Ksiazek, T.G., Gonzalez, J.P. \& Leroy, E.M. (2007) Spatial and temporal patterns of Zaire ebolavirus antibody prevalence in the possible reservoir bat species. Journal of Infectious Diseases, 196, S176-S183.

Proudman, C.J. \& Edwards, G.B. (1992) Validation of a centrifugation/flotation technique for the diagnosis of equine cestodiasis. Veterinary Record 131, 71-72.

Pryor, S.C., Nieto, F., Henry, S. \& Sarfo, J. (2007) The effect of opiates and opiate antagonists on heat latency response in the parasitic nematode Ascaris suum. Life Sciences, 80, 1650-1655.

Pullan, R. \& Brooker, S. (2008) The health impact of polyparasitism in humans: Are we underestimating the burden of parasitic diseases? Parasitology, 135, 783-794.

Ractliffe, L.H. \& Lejambre, L.F. (1971) Increase of rate of egg production with growth in some intestinal nematodes of sheep and horses. International Journal for Parasitology, 1, 
153-156.

Randall, D.A., Williams, S.D., Kuzmin, I.V., Rupprecht, C.E., Tallents, L.A., Tefera, Z., Argaw, K., Shiferaw, F., Knobel, D.L., Sillero-Zubiri, C. \& Laurenson, M.K. (2004) Rabies in endangered Ethiopian wolves. Emerging Infectious Diseases, 10, 2214-2217.

Read, A.F. (1994) The evolution of virulence. Trends in Microbiology, 2, 73-76.

Reece, S.E., Pollitt, L.C., Colegrave, N. \& Gardner, A. (2011) The meaning of death: Evolution and ecology of apoptosis in protozoan parasites. PLoS Pathogens, 7, e1002320.

Reinemeyer, C.R. \& Nielsen, M.K. (2014) Review of the biology and control of Oxyuris equi. Equine Veterinary Education, 26, 584-591.

Reuter, H.-O. \& Adcock, K. (1998) Standardised body condition scoring system for black rhinooceros (Diceros bicornis). Pachyderm, 26, 116-120.

Rieu, E., Recca, A., Bénet, J.J., Saana, M., Dorchies, P. \& Guillot, J. (2007) Reliability of coprological diagnosis of Paramphistomum sp. infection in cows. Veterinary Parasitology, 146, 249-253.

Rinaldi, L., Veneziano, V., Morgoglione, M.E., Pennacchio, S., Santaniello, M., Schioppi, M., Musella, V., Fedele, V. \& Cringoli, G. (2009) Is gastrointestinal strongyle faecal egg count influenced by hour of sample collection and worm burden in goats? Veterinary Parasitology, 163, 81-86.

Roberts, J.L. \& Swan, R.A. (1981) Quantitative studies of ovine haemonchosis. I. Relationship between faecal egg counts and total worm counts. Veterinary Parasitology, 8, 165-171.

Roeder, P., Mariner, J. \& Kock, R. (2013) Rinderpest: the veterinary perspective on eradication. Philosophical transactions of the Royal Society of London. Series B, Biological sciences, 368, 20120139.

Roepstorff, A., BjØrn, H., Nansen, P., Barnes, E.H. \& Christensen, C.M. (1996) Experimental Oesophagostomum dentatum infections in the pig: Worm populations resulting from trickle infections with three dose levels of larvae. International Journal for Parasitology, 26, 399-408.

Rookmaaker, L.C. (2005) The black rhino needs a taxonomic revision for sound conservation. International Zoo News, 52, 280-282.

Round, M.C. (1964) A new species of Stephanofilaria in skin lesions from the black rhinoceros (Diceros bicornis L.) in Kenya. Journal of Helminthology, 38, 87-96.

Rózsa, L., Reiczigel, J. \& Majoros, G. (2000) Quantifying parasites in samples of hosts. Journal of Parasitology, 86, 228-232.

Rudgers, J.A., Miller, T.E.X., Ziegler, S.M. \& Craven, K.D. (2012) There are many ways to be a mutualist: Endophytic fungus reduces plant survival but increases population growth. Ecology, 93, 565-574.

Ruiz, L.A. \& Madrid, V.J. (1992) Studies on the biology of the parasitic isopod Cymothoa exigua Schioedte and Meinert, 1884 and its relationship with the snapper Lutjanus peru (Pisces: Lutjanidae) Nichols and Murphy, 1922, from commercial catch in Michoacan. Ciencias Marinas, 18, 19-34.

Sainsbury, A.W., Deaville, R., Lawson, B., Cooley, W.A., Farelly, S.S.J., Stack, M.J., Duff, P., McInnes, C.J., Gurnell, J., Russell, P.H., Rushton, S.P., Pfeiffer, D.U., Nettleton, P. \& Lurz, P.W.W. (2008) Poxviral disease in red squirrels sciurus vulgaris in the UK: Spatial 
and temporal trends of an emerging threat. EcoHealth, 5, 305-316.

Sainsbury, A.W. \& Vaughan-Higgins, R.J. (2012) Analyzing Disease Risks Associated with Translocations. Conservation Biology, 26, 442-452.

Schalk, G., Forbcs, M.R., Schalk, G. \& Forbes, M.R. (1997) Male biases in parasitism of mammals: Effects of study type, host age, and parasite taxon. Oikos, 78, 67-74.

Schamber, J.L., Esler, D. \& Flint, P.L. (2009) Evaluating the validity of using unverified indices of body condition. Journal of Avian Biology, 40, 49-56.

Schmidt, G.D. \& Roberts, L.S. (2005) Foundations of Parasitology, Seventh Edition edn. McGraw-Hill, New York.

Schulte-Hostedde, A.I. \& Elsasser, S.C. (2011) Spleen mass, body condition, and parasite load in male American mink (Neovison vison). Journal of Mammalogy, 92, 221-226.

Schulte-Hostedde, A.I., Zinner, B., Millar, J.S. \& Hickling, G.J. (2005) Restitution of masssize residuals: Validating body condition indices. Ecology, 86, 155-163.

Schulz, K.C.A. \& Kluge, E.B. (1960) Dermatitis in the black rhinoceros (Diceros bicornis) due to filariasis. Journal of the South African veterinary medicine Association, 31, 265-269.

Schultz, S. R., Barry, R. X., Johnson, M. K., Miller, J. E. \& Forbers, W. A. (1994) Effects of feed plots on fecal egg counts of white-tailed deer. Small Ruminant Research, 13, 9397.

Schwanz, L.E. (2008) Persistent effects of maternal parasitic infection on offspring fitness: implications for adaptive reproductive strategies when parasitized. Functional Ecology, 22, 691-698.

Scott, M.E. (1988) The Impact of Infection and Disease on Animal Populations: Implications for Conservation Biology. Conservation Biology, 2, 40-56.

Seivwright, L.J., Redpath, S.M., Mougeot, F., Watt, L. \& Hudson, P.J. (2004) Faecal egg counts provide a reliable measure of Trichostrongylus tenuis intensities in free-living red grouse Lagopus lagopus scoticus. Journal of Helminthology, 78, 69-76.

Selgrade, M.K. (2007) Immunotoxicity - The risk is real. Toxicological Sciences, 100, 328-332.

Shaw, D.J., Grenfell, B.T. \& Dobson, A.P. (1998) Patterns of macroparasite aggregation in wildlife host populations. Parasitology, 117, 597-608.

Sheldon, B.C. \& Verhulst, S. (1996) Ecological immunology: Costly parasite defences and trade-offs in evolutionary ecology. Trends in Ecology and Evolution, 11, 317-321.

Shim, E. \& Galvani, A.P. (2009) Evolutionary repercussions of avian culling on host resistance and influenza virulence. PLoS ONE, 4, e5503.

Sinniah, B. (1982) Daily egg production of Ascaris lumbricoides: The distribution of eggs in the faeces and the variability of egg counts. Parasitology, 84, 167-175.

Sinniah, B., Sinniah, D. \& Subramaniam, K. (1981) Application of a 'standardized factor' to egg counting techniques for better prediction of worm burdens. Journal of Helminthology, 55, 279-285.

Sithithaworn, P., Tesana, S., Pipitgool, V., Kaewkes, S., Pairojkul, C., Sripa, B., Paupairoj, A. \& Thaiklar, K. (1991) Relationship between faecal egg count and worm burden of Opisthorchis viverrini in human autopsy cases. Parasitology, 102, 277-281.

Skerratt, L.F., Berger, L., Speare, R., Cashins, S., McDonald, K.R., Phillott, A.D., Hines, H.B. \& Kenyon, N. (2007) Spread of chytridiomycosis has caused the rapid global decline 
and extinction of frogs. EcoHealth, 4, 125-134.

Skorping, A., Read, A.F. \& Keymer, A.E. (1991) Life history covariation in intestinal nematodes of mammals. Oikos, 60, 365-372.

Smith, H.J. (1979) Probstmayria vivipara pinworms in ponies. Canadian Journal of Veterinary Research, 43, 341-342.

Smith, K.F., Acevedo-Whitehouse, K. \& Pedersen, A.B. (2009) The role of infectious diseases in biological conservation. Animal Conservation, 12, 1-12.

Smith, L.A., White, P.C.L., Marion, G. \& Hutchings, M.R. (2009) Livestock grazing behavior and inter- versus intraspecific disease risk via the fecal-oral route. Behavioral Ecology, 20, 426-432.

Sorenson, M.D. (1997) Effects of intra- and interspecific brood parasitism on a precocial host, the canvasback, Aythya valisineria. Behavioral Ecology, 8, 153-161.

Spratt, D.M. (1997) Endoparasite control strategies: Implications for biodiversity of native fauna. International Journal for Parasitology, 27, 173-180.

Stear, M. J., Bishop, S.C., Duncan, J.L., McKellar, Q.A. \& Murray, M. (1995) The repeatability of faecal egg counts, peripheral eosinophil counts, and plasma pepsinogen concentrations during deliberate infections with Ostertagia circumcincta. International Journal for Parasitology 25, 375-380.

Stear, M.J., Fitton, L., Innocent, G.T., Murphy, L., Rennie, K. \& Matthews, L. (2007) The dynamic influence of genetic variation on the susceptibility of sheep to gastrointestinal nematode infection. Journal of the Royal Society Interface, 4, 767-776.

Stien, A., Irvine, R.J., Ropstad, E., Halvorsen, O., Langvatn, R. \& Albon, S.D. (2002) The impact of gastrointestinal nematodes on wild reindeer: Experimental and crosssectional studies. Journal of Animal Ecology, 71, 937-945.

Stien, A., Voutilainen, L., Haukisalmi, V., Fuglei, E., Mørk, T., Yoccoz, N.G., Ims, R.A. \& Henttonen, H. (2010) Intestinal parasites of the Arctic fox in relation to the abundance and distribution of intermediate hosts. Parasitology, 137, 149-157.

Strong, D.R. (1986) Density-vague population change. Trends in Ecology and Evolution, 1, 3942.

Sung, G.H., Hywel-Jones, N.L., Sung, J.M., Luangsa-ard, J.J., Shrestha, B. \& Spatafora, J.W. (2007) Phylogenetic classification of Cordyceps and the clavicipitaceous fungi. Studies in Mycology, 57, 5-59.

Swarnkar, C.P., Khan, F.A., Jayasankar, J., Singh, D. \& Bhagwan, P.S.K. (2000) Repeatability of faecal egg-count and haematological values in sheep experimentally infected with Haemonchus contortus. Indian Journal of Animal Sciences, 70, 792-796.

Swinnerton, K.J., Greenwood, A.G., Chapman, R.E. \& Jones, C.G. (2005) The incidence of the parasitic disease trichomoniasis and its treatment in reintroduced and wild Pink Pigeons Columba mayeri. Ibis, 147, 772-782.

Taylor, W.P., Bhat, P.N. \& Nanda, Y.P. (1995) The principles and practice of rinderpest eradication. Veterinary Microbiology, 44, 359-367.

Thomas, F., Poulin, R., Guégan, J.F., Michalakis, Y. \& Renaud, F. (2000) Are there pros as well as cons to being parasitized? Parasitology Today, 16, 533-536.

Thurber, M.I., O'Connell-Rodwell, C.E., Turner, W.C., Nambandi, K., Kinzley, C., Rodwell, 
T.C., Faulkner, C.T., Felt, S.A. \& Bouley, D.M. (2011) Effects of rainfall, host demography, and musth on strongyle fecal egg counts in African elephants (Loxodonta africana) in Namibia. Journal of Wildlife Diseases, 47, 172-181.

Tompkins, D.M., Dunn, A.M., Smith, M.J. \& Telfer, S. (2011) Wildlife diseases: From individuals to ecosystems. Journal of Animal Ecology, 80, 19-38.

Torgerson, P.R., Paul, M. \& Lewis, F.I. (2012) The contribution of simple random sampling to observed variations in faecal egg counts. Veterinary Parasitology, 188, 397-401.

Traversa, D., Fichi, G., Campigli, M., Rondolotti, A., Iorio, R., Proudman, C. J., Pellegrini, D. \& Perrucci, S. (2008). "A comparison of coprological, serological and molecular methods for the diagnosis of horse infection with Anoplocephala perfoliata (Cestoda, Cyclophyllidea)." Veterinary Parasitology 152(3-4): 271-277.

Tremlett, J.G. (1964) Observations on the pathology of lesions associated with Stephanofilaria dinniki Round, 1964 from the black rhinoceros (Diceros bicornis). Journal of Helminthology, 31, 171-174.

Van Nieuwenhuizen, L.C., Verster, A.J.M., Horak, I.G., Krecek, R.C. \& Grimbeek, J.R. (1994) The seasonal abundance of oribatid mites (Acari: Cryptostigmata) on an irrigated Kikuyu grass pasture. Experimental and Applied Acarology, 18, 73-86.

Weldon, C., Du Preez, L.H., Hyatt, A.D., Muller, R. \& Speare, R. (2004) Origin of the amphibian chytrid fungus. Emerging Infectious Diseases, 10, 2100-2105.

Western, D. \& Vigne, L. (1985) The deteriorating status of African rhinos. ORYX, 19, 215-220.

Whiteman, N.K. \& Parker, P.G. (2005) Using parasites to infer host population history: A new rationale for parasite conservation. Animal Conservation, 8, 175-181.

Williamson, R.M., Beveridge, I. \& Gasser, R.B. (1998) Coprological methods for the diagnosis of Anoplocephala perfoliata infection of the horse. Australian Veterinary Journal, 76, 618-621.

Williamson, R.M.C., Gasser, R.B., Middleton, D. \& Beveridge, I. (1997) The distribution of Anoplocephala perfoliata in the intestine of the horse and associated pathological changes. Veterinary Parasitology, 73, 225-241.

Wilson, S. (2001) Body size influences on the foraging behaviour of the thicket biome browsers $\mathrm{PhD}$ Thesis, University of Port Elizabeth.

Wobeser, G. (2002) Disease management strategies for wildlife. OIE Revue Scientifique et Technique, 21, 159-178.

Wood, E.L., Matthews, J.B., Stephenson, S., Slote, M. \& Nussey, D.H. (2013) Variation in fecal egg counts in horses managed for conservation purposes: Individual egg shedding consistency, age effects and seasonal variation. Parasitology, 140, 115-128.

Woodhams, D.C. \& Alford, R.A. (2005) Ecology of chytridiomycosis in rainforest stream frog assemblages of tropical Queensland. Conservation Biology, 19, 1449-1459.

Woodroffe, R. (1999) Managing disease threats to wild mammals. Animal Conservation, 2, 185-193.

Woolhouse, M.E.J. (1998) Patterns in parasite epidemiology: The peak shift. Parasitology Today, 14, 428-434.

Woolhouse, M.E.J., Dye, C., Etard, J.F., Smith, T., Charlwood, J.D., Garnett, G.P., Hagan, P., Hii, J.L.K., Ndhlovu, P.D., Quinnell, R.J., Watts, C.H., Chandiwana, S.K. \& Anderson, 
R.M. (1997) Heterogeneities in the transmission of infectious agents: Implications for the design of control programs. Proceedings of the National Academy of Sciences of the United States of America, 94, 338-342.

Wormser, G.P., Dattwyler, R.J., Shapiro, E.D., Halperin, J.J., Steere, A.C., Klempner, M.S., Krause, P.J., Bakken, J.S., Strle, F., Stanek, G., Bockenstedt, L., Fish, D., Dumler, J.S. \& Nadelman, R.B. (2006) The clinical assessments treatment, and prevention of lyme disease, human granulocytic anaplasmosis, and babesiosis: Clinical practice guidelines by the Infectious Diseases Society of America. Clinical Infectious Diseases, 43, 10891134.

Wright, A.N. \& Gompper, M.E. (2005) Altered parasite assemblages in raccoons in response to manipulated resource availability. Oecologia, 144, 148-156.

Yamamura, N. (1993) Vertical Transmission and Evolution of Mutualism from Parasitism. Theoretical Population Biology, 44, 95-109.

Yanoviak, S.P., Kaspari, M., Dudley, R. \& Poinar Jr, G. (2008) Parasite-induced fruit mimicry in a tropical canopy ant. American Naturalist, 171, 536-544.

Zajac, A.M. \& Conboy, G.A. (2006) Veterinary Clinical Parasitology, 7th edn. Blackwell Publishing, Oxford.

Zimmermann, D.E. (2009) The occurrence of piroplasms in various South African black rhinoceros (Diceros bicornis) populations. M.Sc. Thesis Faculty of Veterinary Sciences, University of Pretoria, 1-39.

Zug, R. \& Hammerstein, P. (2014) Bad guys turned nice? A critical assessment of Wolbachia mutualisms in arthropod hosts. Biological Reviews.

Zumpt, F. (1964) Parasites of the white and black rhinoceroses. Lammergeyer, 3, 59-70, figs. 51-12.

Zuur, A.F., Ieno, E.N., Walker, N.J., Saveliev, A.A. \& Smith, G.M. (2009) Mixed Effects Models and Extensions in Ecology with R. Springer Science+Business Media 

Appendix 1: The effect of host capture on parasites

\section{Appendix 1: The effect of host capture on faecal egg counts}

\section{Introduction}

The translocation and reintroduction of large mammals is an extraordinary process. Since 1962 it has been used extensively to expand the black rhinoceros population in South Africa (Hitchins, 1984). For instance, 682 animals were captured from South African reserves between 1981 and 2005 (Linklater et al., 2012). These animals were either used to create new populations of black rhinoceros or reinforce existing populations.

The success of translocated black rhinoceros is highly variable, with just over $10 \%$ of individuals dying during the first year after their release (Linklater et al., 2012). A soft release method is often used, which brings individuals into captivity for a period of time before release. During the first weeks after being brought into captivity many individuals feed poorly and lose weight. This is a highly stressful time for the captured individual, having to adapt to captivity, new food sources, and daily contact with people (Linklater et al., 2010). However, after a period of acclimatisation individuals are thought to start putting on body weight, fat reserves, and improve in condition (pers. comm. C. Dreyer).

How parasites are affected and react to host capture has not, to my knowledge, been previously documented. During the spring of 2011, six rhinoceros were brought into captivity as part of a soft-release program in the Eastern Cape of South Africa. The opportunity was taken to monitor the faecal egg counts of black rhinoceros brought into captivity. This was an opportunistic observational study. As numerous potential factors may influence any observed results, and sample size was small, only a descriptive interpretation of results was planned.

\section{Methods}

In November 2011, six rhinoceros were brought into captivity for a soft release into a new reserve. To capture a rhinoceros, the individual is darted from the air using the potent opioid M99, also known as Etorphine. It takes approximately three minutes for the sedative to take effect. During this time, as the sedative takes effect, the rhinoceros is chased towards the nearest road or accessible location, by helicopter or people on foot or horseback. Once the rhinoceros is sedated ground crews are directed in by the helicopter. A health check is immediately performed. A vehicle with a crate is then manoeuvred into close proximity of the rhinoceros. A rope is tied around the head of the rhinoceros, and a brake rope attached it its rear 
foot. The rhinoceros is partially revived, and encouraged to walk into the crate. The crate is then used for transport before the rhinoceros is released into a holding enclosure known as a boma, either at the site of capture or release. Individuals were held for 30-32 days in captivity before release.

It is thought to take a number of hours for the full effect of the sedation to wear off (King, 1969). During the first few days in captivity black rhinoceros rarely eat and drink (pers. comm. C. Dreyer). In this case they were supplied with fresh water, lucerne (also known as alfafa, Medicago sativa), and Euphorbia bothae, a highly preferred food species, along with a mix of branches from other known preferred species. Some individuals fail altogether to adapt to captive conditions. In these cases, where rhinoceros continue to reject supplied food and water, they are released. Individuals lose condition over approximately the first two weeks of captivity, and then usually start to regain body weight and condition.

The first faecal sample was taken from the rectum in the field at the site of capture when the animals were under heavy sedation. Subsequent samples were taken from the floor of the boma during the rhinoceros's period in captivity.

Bomas could be divided into two compartments, so that one could be cleaned while the rhinoceros was secured in the other. Cleaning took place every morning at dawn. During this time fresh faecal samples were collected from each rhinoceros. Freshness of the sample could usually be ascertained by the residual heat within the sample. Samples were taken from the warmest, most recently deposited samples each morning.

Enclosures are not cleaned during the first days of confinement to reduce stress. Hence, the first sample from enclosed individuals was taken 9-10 days after capture. Due to the somewhat surprising nature of the first result, resampling occurred the following day to confirm the result. Subsequent samples were taken every 4-7 days thereafter, the exact day was determined by logistical constraints. Individuals were captured on different dates, however all faecal samples from captive animals were taken on the same date, meaning samples represent slightly different times spent in captivity (Figure A1). The final sample of the monitoring was taken on the day the individual was removed from the boma. Faecal egg counts were performed as outlined in Chapter 2. 


\section{Results}

Rectal faecal samples could be obtained for 5 out of 6 captured individuals. A rectal faecal sample could not be obtained from one individual due to a lack of faeces in the rectum at the time of capture. Enclosures are not cleaned for a number of days following the release of individuals into the boma to help with acclimatisation, hence an initial representative sample for this individual could not be obtained. One rhinoceros was released shortly after capture, as it had not adapted well to the captive environment.

For strongyles, three out of four rhinoceros showed a large drop in faecal egg output following capture (Figure A1). For these individuals, FECs dropped from a mean of 6167 epg $(\mathrm{SD}=1610)$ in rectal faecal samples to a mean of $225 \mathrm{epg}(\mathrm{SD}=43)$ in samples collected $\sim 11$ days after capture. The egg count from the fourth individual increased from 1275 epg to 2275 epg over the same period.

For Anoplocephala sp., a similar trend was observed as two out of three rhinoceros infected with the species showed a drop in FECs. Egg counts dropped from 325 and 125 epg, to 25 and 0 epg respectively (Figure A1).
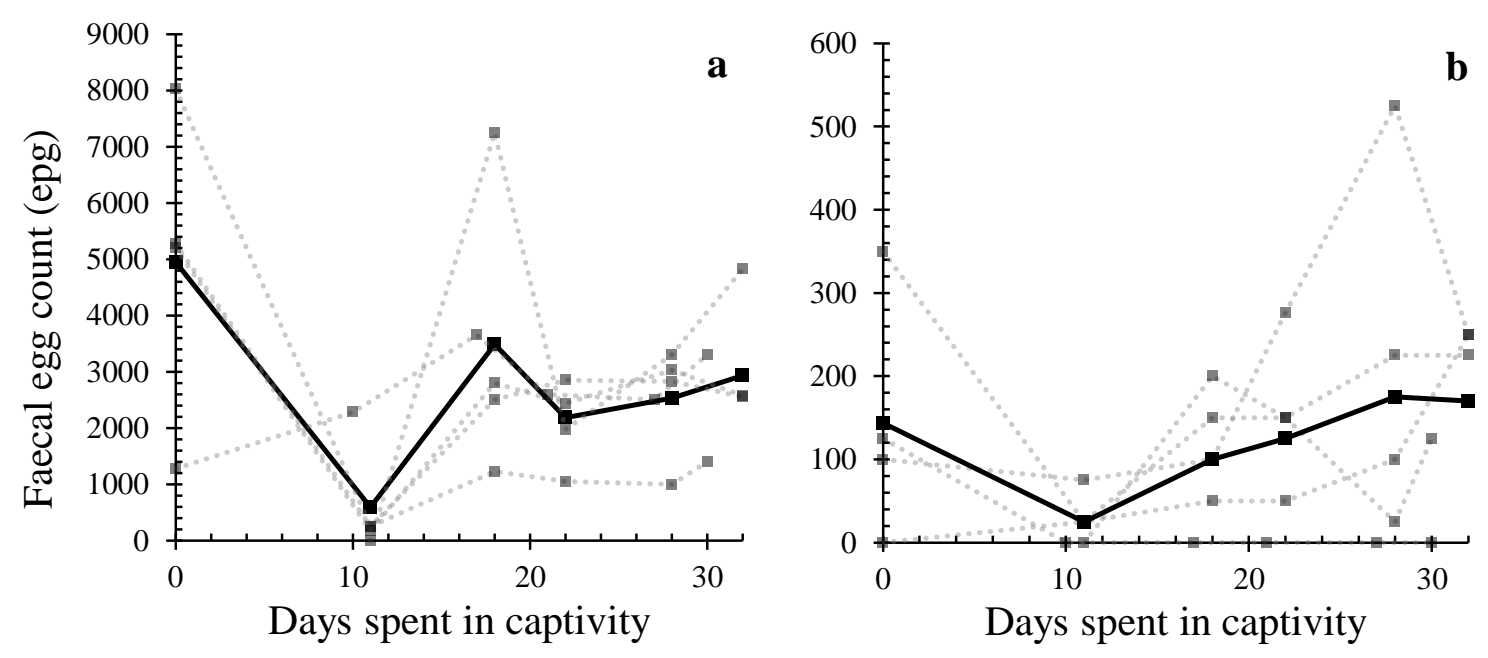

Figure A1 Faecal egg counts taken from samples collected from black rhinoceros brought into captivity. The day of sample collection is the number of days since capture. Mean, solid lines utilise points collected on the same date, which is different from days spent in captivity. A) Strongyles, b) Anoplocephala sp. 


\section{Discussion}

An initial reasoning for this drop was thought to be differences between the egg counts of rectal samples, and freshly deposited faeces. However, the mean FECs from the same population sampled using deposited faeces, 3289 epg (Chapter 3), is slightly higher than the mean FECs from those taken rectally, 2987 epg (Chapter 4). Also, FECs recovered somewhat as time in captivity progressed.

Strong conclusions cannot be made from this small sample study. Nevertheless, the study does raise questions about the impact of host capture on parasite populations. This is worthy of future investigation, particularly why parasite egg counts might decline in some individuals but not others after capture.

There may be a wide variety of explanations for the possible decline in egg output following capture for certain individuals and I outline some interesting hypotheses that could be tested in the future. A decline in egg output may be a result of reduced parasite egg output per individual parasite, or a reduction in parasite load. Both are possible as the timeframe of the recovery in egg production may have been caused by new parasite infection (although the lifecycles of the parasites have not been directly studied). For strongyles this is less likely as development within the host will likely take a number of weeks. However, tapeworm development within the host may be much quicker (Schmidt and Roberts, 2005). Hence, it is possible that some or all parasites were killed at capture. Hypotheses for the observed drop in FECs may include:

- Black rhinoceros ingest very little water and food during their first days in captivity. This may radically alter the intestinal environment, depressing parasite populations, or reducing egg output.

- Parasite egg output is often seasonal, and in long-lived parasite species this may be to maximise egg output during times when transmission is most likely (Baudena et al., 2000, Nalubamba et al., 2012). All food was cut before being fed to rhinoceros, or was dried lucerne. Hence, the water content of food may be low. This may be an indication to intestinal parasites that external transmission conditions are poor, and hence in response parasites may lower egg output.

- The anaesthetic used to sedate black rhinoceros is a powerful opioid, thought to be 1000 3000 times more potent than morphine and fatal to humans (Bentley and Hardy, 1967). 
Nematodes have been shown to be sensitive to opiates (Pryor et al., 2007), hence they may have been effected, assuming the opioid could reach them.

- Euphorbia bothae is a highly preferred food species of black rhinoceros. Captured individuals were fed E. bothae harvested from a neighbouring area. Although, food intake was thought to be low during the first few days after capture, as a highly preferred species E. bothae may have been ingested. Latex from Euphorbia spp has previously been found to have some anti-parasitic properties (Mazoir et al., 2011).

- During times of high host stress it would be beneficial for a parasite to reduce their burden, particularly if the host is at imminent risk (1.2.2). As capture is an extraordinarily stressful event, parasites may be reducing their burden to increase host longevity, and this is reflected in low levels of egg production.

Here I have provided some possible explanations as to the possible drop in FECs observed in captured black rhinoceros. In the future, a study may wish to investigate whether the effect can be reproduced, and which of the discussed factors may be influential. For black rhinoceros a study would have to test hypotheses within the limits imposed by conservation management. Primarily, a study with a larger sample size would be beneficial, which identified whether the capture process, or the confinement in captivity has any effect on parasites. This could be reasonably easily done by comparing FECs from individuals directly released without a period in captivity, with those captured for soft release. Alternatively, an organism other than black rhinoceros may provide an opportunity for a much larger sample size and greater opportunity for manipulating capture conditions. 



\title{
Appendix 2:
}

\begin{tabular}{cc|}
\hline & Contents lists available at Sciencedirect \\
\hline ELSEVIER & International Journal for Parasitology: \\
Parasites and Wildlife & journal homepage: www.elsevier.com/locate/ijppaw \\
\hline
\end{tabular}

\section{Reducing sampling error in faecal egg counts from black rhinoceros (Diceros bicornis)}

\author{
Andrew P. Stringer ${ }^{a, *}$, Diane Smith ${ }^{b}$, Graham I.H. Kerley ${ }^{b}$, Wayne L. Linklater ${ }^{a, b}$ \\ ${ }^{a}$ Centre for Biodiversity and Restoration Ecology, Victoria University of Wellington, New Zealand \\ ${ }^{\mathrm{b}}$ Centre for African Conservation Ecology, Nelson Mandela Metropolitan University, Port Elizabeth, South Africa
}

\section{A R T I C L E I N F O}

\section{Article history:}

Received 26 August 2013

Revised 7 October 2013

Accepted 8 October 2013

\section{Keywords:}

Faecal egg counts

Parasitology

Sampling error

Black rhinoceros

\begin{abstract}
A B S T R A C T
Faecal egg counts (FECs) are commonly used for the non-invasive assessment of parasite load within hosts. Sources of error, however, have been identified in laboratory techniques and sample storage. Here we focus on sampling error. We test whether a delay in sample collection can affect FECs, and estimate the number of samples needed to reliably assess mean parasite abundance within a host population. Two commonly found parasite eggs in black rhinoceros (Diceros bicornis) dung, strongyle-type nematodes and Anoplocephala gigantea, were used. We find that collection of dung from the centre of faecal boluses up to six hours after defecation does not affect FECs. More than nine samples were needed to greatly improve confidence intervals of the estimated mean parasite abundance within a host population. These results should improve the cost-effectiveness and efficiency of sampling regimes, and support the usefulness of FECs when used for the non-invasive assessment of parasite abundance in black rhinoceros populations.
\end{abstract}

(c) 2013 The Authors. Published by Elsevier Ltd. Open access under CC BY license.

\section{Introduction}

Evaluating intestinal helminth infections is important for animal production and animal welfare. Parasites may impact population growth (Irvine, 2006) and so the abundance of parasites within populations and across meta-populations is important for the conservation of threatened species. Parasites are usually aggregated within a small proportion of a host population (Poulin 2007). Gasbarre et al. (1996) concludes that greater than 15 samples are needed to ensure that $95 \%$ of the time the level of parasite aggregation is accurately estimated. Thus, large sample sizes are often needed to accurately capture the level of parasite abundance within a population.

In conservation management, culling an animal to determine its parasite intensity is rarely an option. Opportunistic autopsies on natural deaths can be useful but may give a biased sample of a population's parasite abundance. For some intestinal parasites, faecal egg counts (FECs) are used as a non-invasive tool to determine the abundance of parasites within a host. The technique is

\footnotetext{
* Corresponding author. Address: School of Biological Sciences, Victoria University of Wellington, PO Box 600 , Wellington 6140 , New Zealand. Tel.: +64 4463 5339.
}

E-mail addresses: Andrew.Stringer@vuw.ac.nz, stringea@tcd.ie (A.P. Stringer). particularly suitable for conservation mangers because it allows extensive and intensive non-invasive sampling of individual hosts and populations of hosts. Nevertheless, the technique does have constraints.

A direct and positive relationship between FECs and parasite burdens has been shown in numerous studies (Daş et al. 2011; McKenna, 1981; Rieu et al., 2007; Roberts and Swan, 1981; Seivwright et al., 2004; Sinniah, 1982; Sinniah et al., 1981; Sithithaworn et al., 1991). However an often cited concern with FECs is that this relationship may not be linear (Gillespie, 2006; Gooderham and Schulte-Hostedde, 2011). For instance, at lower parasite densities a sex-ratio bias towards female parasites may increase FECs independently of actual parasite population sizes (Poulin, 1997). Conversely, at high parasite densities, parasite ovulation rate may be reduced due to inter-specific competition (Christensen et al., 1995; Roepstorff et al., 1996; Sithithaworn et al., 1991). These patterns of variation must be considered during the interpretation of FECs but are not a source of methodological error.

It is recommended that FECs should only be used when samples have been taken rectally or directly after observed defecation (Zajac and Conboy, 2006). This is because temperature, light levels and oxygen availability may all be cues for the hatching of directly-transmitted parasites post-defecation (Nielsen et al., 2010). Furthermore the moisture content of faeces may change rapidly or eggs may be eaten by predators, thereby affecting the eggs per gram of faeces estimate (Anderson and Schad, 1985 
Eberhard et al., 2001). These mechanisms may also differentially influence the FEC within the dung bolus, such as in the surface layer of dung in comparison to the centre, as the surface layer of dung is more exposed to the environment (Daş et al., 2011).

FECs are frequently used to evaluate the abundance of parasites within a host population. However, they are rarely used with elusive host species of conservation concern such as the black rhinoceros (Diceros bicornis). Known sources of methodological error come from laboratory techniques (Cringoli et al., 2004) and sample storage strategies (Dacombe et al., 2007; Seivwright et al., 2004; Zajac and Conboy, 2006). This paper will focus on potential sources of sampling error, primarily the collection of dung where defecation has not been observed. This paper investigates how sampling at known intervals following defecation, and from different locations within the faecal bolus, affects FECs from black rhinoceros. We also determine the minimum number of samples needed to accurately estimate mean parasite abundance within a population.

\section{Materials and methods}

FECs were performed on black rhinoceros (Diceros bicornis) dung. Two types of parasite eggs were commonly found, strongyle-type eggs (family Strongylidae), and a cestode, Anoplocephala sp. There are seven species of strongyle that infect black rhino in South Africa (Kiluluma spp. and Khalilia rhinocerotis), and only one cestode, Anoplocephala gigantea (Penzhorn et al., 1994; Knapp et al., 1997).

To test for differences between the centre and the surface layer of faecal boluses, 43 fresh boluses were sampled over four two-day sampling periods spread between Apr-Sep in 2011 from a wildlife reserve in the Eastern Cape of South Africa. Fresh faeces were collected during the early morning (dawn-10am) from latrines located along roads. To reduce the possibility of pseudoreplication a stratified random sampling regime, modified to ensure a minimum of $1 \mathrm{~km}$ between sample sites, was used. Black rhinoceros boluses are $12-15 \mathrm{~cm}$ in diameter. A sample of approximately $10 \mathrm{~g}$ of dung was collected from the centre of one complete bolus per dung pile. From the same bolus, another sample of approximately $10 \mathrm{~g}$ of dung was taken from the surface to a maximum depth of $1 \mathrm{~cm}$. Each sample was stored at $4{ }^{\circ} \mathrm{C}$ in a sealed plastic bag, with excess air removed, until analysis (within 4-28 h) (Nielsen et al., 2010). A modified McMaster technique (Zajac and Conboy, 2006) using Sheather's sugar solution was used for the flotation and enumeration of parasite eggs. Four replicate chambers were counted for each sample resulting in an analytical sensitivity of 25 eggs per gram (epg) of faeces per individual. Paired t-tests were used to compare differences between the surface layer and centre of each bolus sampled. The differences between these samples were normally distributed. SPSS (IBM, 2011) was used for all calculations unless otherwise stated.

To test how FECs may change due to a delay in sample collection, freshly deposited boluses $(n=7)$ were collected from black rhino captured for translocation and reintroduction, and held temporarily in purpose-built enclosures in the same Eastern Cape reserve. Boluses of dung were collected at dawn before the animals' enclosures were cleaned. Boluses were judged freshly defecated if they were still warm. Boluses were placed outside the enclosures and subjected to normal daytime conditions. Approximately $10 \mathrm{~g}$ samples were taken from the centre of each immediately and then at $3 \mathrm{~h}$ intervals up until $9 \mathrm{~h}$ after initial collection. Boluses were reformed after each sampling event. Samples were stored and analysed as previously indicated. Strongyle egg maturity was estimated based on the internal structure of each egg. Morulated eggs (Zajac and Conboy, 2006) or those with no clear internal structure were classified as immature, while any egg where larvae or a pre-larval shape could be identified inside the egg were classified as mature. With time, strongyle eggs were expected to hatch and not be recoverable using the McMaster technique while A. gigantea eggs were not expected to hatch. Data was normally distributed and Mauchly's sphericity test showed that sphericity could be assumed. We used a repeated measures ANOVA to test whether FECs and the level of egg maturity changed with time after defecation.

We then investigated how sample size affects the reliability of estimates of mean FEC for a population. Fresh faecal samples were collected from 18 populations of black rhinoceros from across eastern South Africa (Stringer, unpublished data), where parasite abundance, aggregation and sample size vary. Bootstrap $90 \%$ confidence interval estimations for the mean FEC using 2000 replications were calculated using the software "Quantitative Parasitology" (Rózsa et al., 2000) for each population. For each population, the size of these confidence intervals (as percentage distances from the mean) were then plotted against sample size. A multiple regression was then used to test how parasite aggregation, estimated mean abundance, and sample size affected confidence intervals. The level of parasite aggregation within a population was calculated using the corrected moment estimation of $k$ which reduces bias caused by small sample size (Gregory and Woolhouse, 1993).

Five wildlife reserves from the previously mentioned dataset with sample sizes larger than nine were used to further investigate the precision of estimates of mean parasite abundance. First, data points were randomised. Then, after each sampling event, mean parasite abundance was calculated. The percentage distance of this mean from the best estimate of the mean using all data points was then calculated.
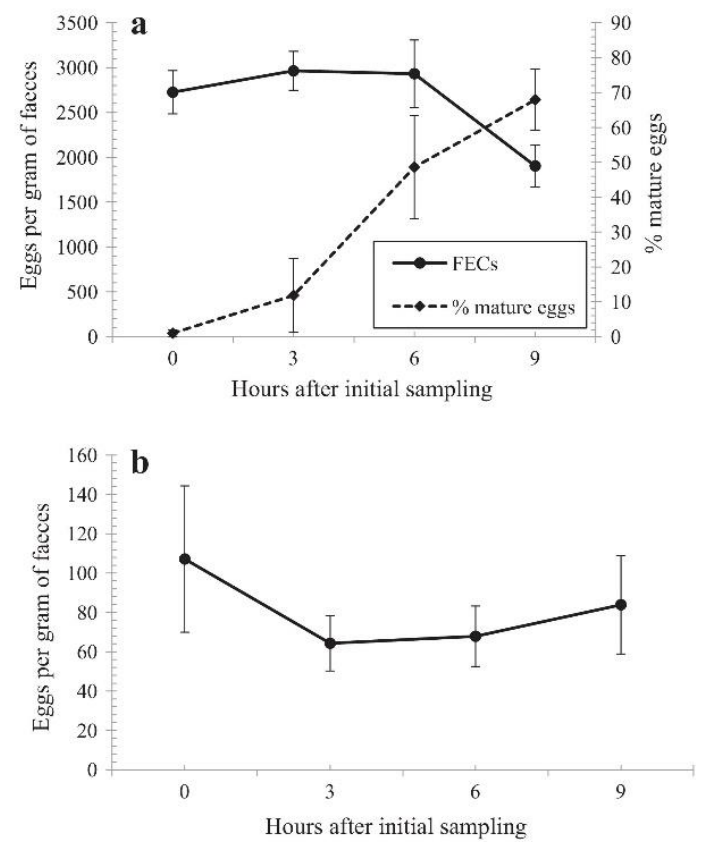

Fig. 1. (a) Mean strongyle FECs and mean percentage of mature eggs, sampled 3, 6 $\& 9 \mathrm{~h}$ after initial collection from captive black rhino. Error bars represent \pm 1 S.E. (b) Mean A. gigantea FECs, sampled 3, $6 \& 9 \mathrm{~h}$ after initial collection from captive black rhino. Error bars represent \pm 1 S.E. 


\section{Results}

FECs from the centre of faecal boluses were significantly higher than from the surface layer of boluses for both parasite groups when all four sampling periods were combined (strongyles: $T_{42}=6.65, p<0.001$. A. gigantea: $T_{42}=3.23, p=0.002$ ). Analysing the data for each sampling period revealed significant differences on three out of four sampling periods for strongyles and two out of four sampling periods for $A$. gigantea.

For the time-specific FEC sampling, there was a significant reduction in strongyle FECs over time (RM ANOVA, $n=7$, $F_{3,18}=5.1, p=0.01$ ), while the percentage of mature eggs increased through time $\left(F_{3,18}=15.1, p<0.001\right)$. FECs did not decline until after $6 \mathrm{~h}$ had passed since defecation and initial collection (see Fig. 1). In a pairwise comparison of time points using a Bonferroni correction, the largest difference was between the 3 and 9 h collection points. A. gigantea FECs were slightly more variable, and did not change significantly through time $\left(F_{3,18}=0.07, p=0.86\right)$.

Lower confidence intervals for mean abundance improved as sample size increased for both parasite groups (see Fig. 2). Similarly, upper confidence intervals improved with increasing sample size for strongyles, but not for A. gigantea (although this trend appears to be driven by a single extreme value). Upper confidence limits were generally much further from the mean. Generally, confidence intervals were much improved when sample size was greater than nine (see Fig. 2).

Further investigation included the impacts of aggregation and calculated mean on the size of confidence intervals. A multiple regression revealed that the size of confidence intervals for strongyles was significantly predicted by parasite aggregation (Beta $=-0.57, p<0.01$ ) and mean abundance (Beta $=-0.61$, $p<0.01$ ) but not sample size (Beta $=-0.11, \mathrm{n} . \mathrm{s}$ ). The overall mode fit was $r^{2}(\mathrm{adj})=0.63$. While, for A.gigantea confidence intervals were significantly predicted by parasite aggregation (Beta $=-0.82$, $p<0.001$ ) but not mean abundance (Beta $=-0.04$, n.s) or sample size (Beta $=-0.21, n . s)$. The overall model fit was $r^{2}(\operatorname{adj})=0.61$.

Using only those reserves with nine or more samples, after six samples, all estimates of mean parasite abundance were within $20 \%$ of the best estimate for strongyles (see Fig. 3a). The population that took longest to improve had the lowest mean abundance. While for A. gigantea, after nine samples 4 out of 5 reserves' estimates of the mean were within $20 \%$ of the best estimate. The single reserve that took longer to improve (in Fig. 3b) is the same extreme result seen in Fig. 2c, and is associated with a high degree of parasite aggregation within that population.

\section{Discussion}

Parasite enumeration in free-ranging wildlife is often difficult and time consuming. There are many potential sources of error that may affect FECs. Identifying these sources of error is important so that they can be controlled by experimental design or considered during the interpretation of results.

Overall FECs were lower in faeces collected from the surface layer of boluses in comparison to the centre of bolus, although this trend differed between sampling periods. It is possible that this variation is caused by exposure to environmental conditions and predation, depleting egg density in the surface layer. Sampling from the centre of boluses may be a useful technique for host species with similar sized, or larger boluses, such as white rhino (Ceratotherium simum) and African elephant (Loxodonta spp.). In

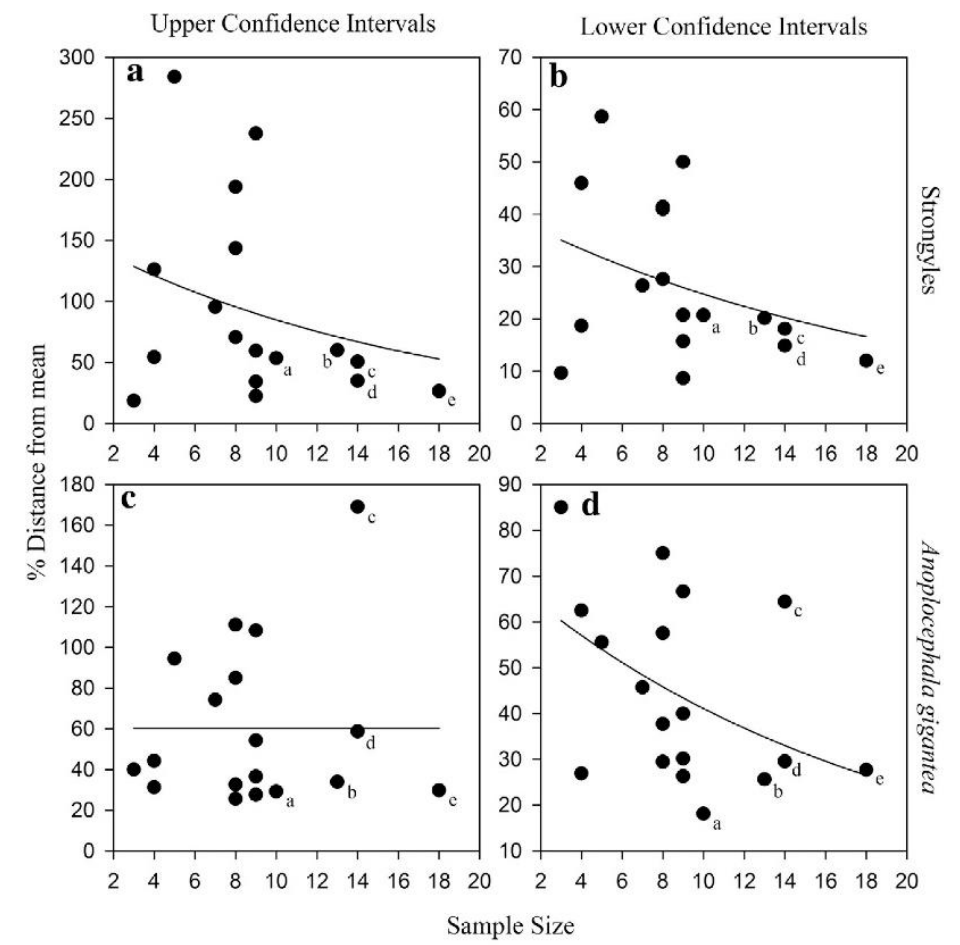

Fig. 2. The distance from the mean of bootstrap $90 \%$ confidence intervals plotted against sample size for FECs for 18 black rhino populations. Lines represent the fitted negative exponential curve. Five populations are labelled "a-e" that are used in Fig. 3. (a) Strongyle upper confidence intervals. (b) Strongyle lower confidence intervals. (c) A. gigantea upper confidence intervals. (d) A. gigantea lower confidence intervals. 
other host ungulate species, with smaller faecal boluses and larger surface area to volume ratios, environmental influences may more quickly penetrate the centre of boluses.

FECs were robust to quite long periods between defecation and sample collection. Strongyle eggs matured during the initial 6 hour period after defecation but this did not affect FECs. A. gigantea FECs did not decline, however these eggs do not develop into larvae as they are instead eaten by their intermediate host. Hence, the number of eggs eaten by intermediate hosts or predators, or decaying up until 9 hours after defecation was minimal. It must be noted that all FECs were calculated by the wet weight of the sample rather than the dry weight. Hence, it would be expected that as water evaporated from the dung calculated FECs would increase. We did not observe any increase in FECs indicating that actua egg numbers may have reduced over time or that evaporation from the centre of dung boluses was minimal.

A sampling regime that collects samples without observing defecation is only useful if faeces can be identified as being collected within 6 hours of defecation. Here, although the rate at which strongyle eggs mature was variable between samples, the level of egg maturation within the sample could be used to broadly assess the age of faeces. There could be other problems with collecting older samples. For instance, mature eggs may be more difficult to identify (Zajac and Conboy, 2006), although this was not found for the parasite groups studied here. Finally, if defecation is no observed, then accidental collection from the same individual would be possible. A stratified random sampling regime would reduce the chance of this pseudoreplication (Dytham, 2003), but not eliminate it.

Collecting more than nine samples greatly improved confidence intervals for the population mean. However, there was a great dea of statistical noise associated with this relationship. Some of this could be explained by the estimated mean - a smaller mean was associated with larger confidence intervals because our methodology had a resolution of 25 epg. Hence, if egg counts are low then the methodological resolution of egg counts should be increased. This may be done by decreasing the dilution factor or increasing the number of replicates from each sample (Torgerson et al., 2012).

A large amount of variation in the size of confidence intervals was explained by the level of parasite aggregation within a population. Hence, if parasite aggregation is high more samples may be required in order to improve confidence intervals. This reflects one of the disadvantages of reporting mean abundance in parasite studies - the mean is dependent on a few heavily infected individuals and is not accurately indicative of the typical infection across the population of hosts (Rózsa et al., 2000). This was shown in $A$. gigantea (Fig. 3b) where, although the accuracy of estimates of mean parasite abundance was positively associated with sample size, certain reserves required many more samples for mean abundance estimates to become accurate. This was caused by a few, heavily parasitized individuals.

Depending on the study aims, the mean level of infection need not be determined at all. For instance, Generalised Linear Mixed Models allow for non-normally distributed data and random effects, hence mitigating the need to reduce each population to a single data point (Bolker et al., 2009). The required sample size is then dependent on the study questions - whether it is the typical level of infection or the heavily parasitized individuals that are of interest.

These results should reduce the costs and labour of data collection, and increase the usefulness of FECs as a tool for the non-invasive assessment of parasite abundance. Although this study was
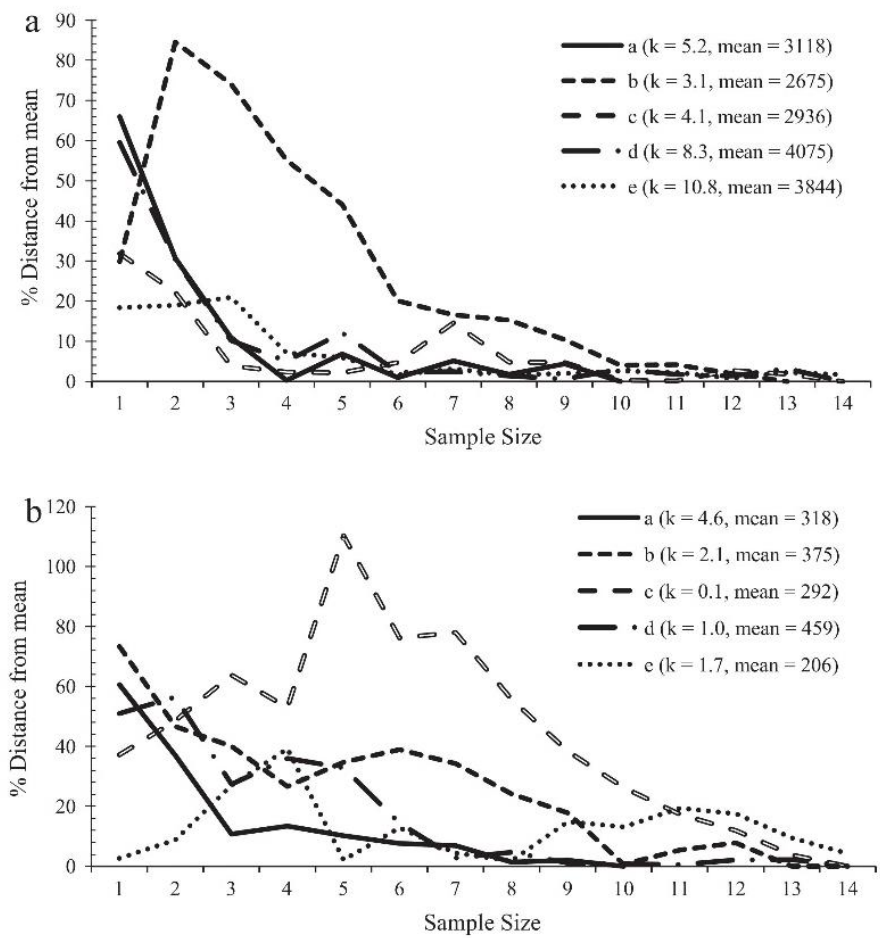

Fig. 3. The accuracy of the estimated mean after each sampling event is plotted for each reserve with 10 or more samples. The corrected moment estimate of $k$ and the population mean are given in the legend. (a) Strongyle (b) A. gigantea 
specific to black rhino, the techniques used here could apply to numerous other host species. However, studies wishing to use a delayed faecal sampling strategy must independently test the speed at which FECs decline. Quick and easy methods of parasite enumeration will assist conservation managers identify when parasites may be of concern for the conservation of threatened species.

\section{Acknowledgements}

Special thanks go to Eastern Cape Parks and Tourism Agency and associated staff, Dr Dean Peinke, Brad Fike, Gav Shaw, Nelson Mandela Metropolitan University, Dr Shirley Parker-Nancy, Simon Johnson, Eric Verburg, Victoria University of Wellington, Roan Plotz, Dr Rosalyn Anderson-Lederer. This project was made possible by funds from the U.S. Fish and Wildlife Service (Grant numbers: 98210-6-G102, 98210-4-G920), a Victoria University of Wellington Doctoral Assistantship and the National Research Foundation (NRF). There are no known conflicts of interest. The animals used in this study were ethically treated and the protocols used were given approval from the Victoria University of Wellington Animal Ethics Committee (ref: 2010R6).

\section{References}

Anderson, R.M., Schad, G.A., 1985. Hookworm burdens and faecal egg counts: an analysis of the biological basis of variation. Transactions of the Royal Society of Tropical Medicine and Hygiene 79, 812-825.

Bolker, B.M., Brooks, M.E., Clark, C.J., Geange, S.W., Poulsen, J.R., Stevens, M.H.H. White, J.S.S., 2009. Generalized linear mixed models: a practical guide for ecology and evolution. Trends in Ecology and Evolution 24, 127-135.

Christensen, C.M., Barnes, E.H., Nansen, P., Roepstorff, A., Slotved, H.C., 1995 Experimental Desophagostomum dentatum infection in the pig: worm populations resulting from single infections with three doses of larvae. populations resulting from single infections with the
International Journal for Parasitology $25,1491-1498$.

Cringoli, G., Rinaldi, L., Veneziano, V., Capelli, G., Scala, A., 2004. The influence of flotation solution, sample dilution and the choice of McMaster slide area flotation solution, sample dilution and the choice of McMaster slide area
(volume) on the reliability of the McMaster technique in estimating the faecal (volume) on the reliability of the McMaster technique in estimating the faecal egg counts of gastrointestinal strongyles
Veterinary Parasitology $123,121-131$

Dacombe, R.J., Crampin, A.C., Floyd, S., Randall, A., Ndhlovu, R., Bickle, Q., Fine, P.E.M., 2007. Time delays between patient and laboratory selectively affect accuracy of helminth diagnosis. Transactions of the Royal Society of Tropica Medicine and Hygiene 101, 140-145.

Daş, G., Savaș, T., Kaufmann, F., Idris, A., Abel, H., Gauly, M., 2011. Precision, repeatability and representative ability of faecal egg counts in Heterakis gallinarum infected chickens. Veterinary Parasitology 183, 87-94.

Dytham, C., 2003. Choosing and Using Statistics: A Biologist's Guide, second ed. Blackwell Science, Oxford.

Eberhard, M.L., Kovacs-Nace, E., Blotkamp, J., Verwij, J.J., Asigri, V.A.A., Polderman, A.M., 2001. Experimental Oesophagostomum bifurcum in monkeys. Journal of Helminthology $75,51-56$.

Gasbarre, L.C., Leighton, E.A., Bryant, D., 1996. Reliability of a single fecal egg pe gram determination as a measure of individual and herd values for trichostrongyle nematodes of cattle. American Journal of Veterinary Research $57,168-171$.

Gillespie, T.R., 2006. Noninvasive assessment of gastrointestinal parasite infections in free-ranging primates. International Journal of Primatology 27, 1129-1143. Gooderham, K., Schulte-Hostedde, A., 2011. Macroparasitism influences reproductive success in red squirrels (Tamiasciurus hudsonicus). Behavioral Ecology 22, 1195-1200

Gregory, R.D., Woolhouse, M.E.J., 1993. Quantification of parasite aggregation: a simulation study. Acta Tropica 54, 131-139.

IBM, 2011. SPSS Statistics, Version 20

Irvine, R.J., 2006. Parasites and the dynamics of wild mammal populations. Anima. Science 82, 775-781.

Knapp, S.E., Krecek, R.C., Horak, I.G., Penzhorn, B.L., 1997. Helminths and arthropods of black and white rhinoceroses in Southern Africa. Journal of Wildlife Diseases 33, 492-502.

McKenna, P.B., 1981. The diagnostic value and interpretation of faecal egg counts in sheep. New Zealand Veterinary Journal 29, 129-132.

Nielsen, M.K., Vidyashankar, A.N., Andersen, U.V., DeLisi, K., Pilegaard, K., Kaplan, R.M., 2010. Effects of fecal collection and storage factors on strongylid egg counts in horses. Veterinary Parasitology 167, 55-61.

Penzhorn, B.L., Krecek, R.C., Horak, I.G., Verster, A.J.M., Walker, J.B., Boomker, J.D.F., Knapp, S.E., Quandt, S.K.F., 1994. Parasites of African rhinos: a documentation. pp. 168-175. In: Penzhorn, B.L. et al. (Eds.), Proceedings of a symposium on rhinos as game ranch animals, 9-10 September 1994, Onderstepoort, Republic of South, Africa, pp. i-iv, 1-242.

Poulin, R., 1997. Population abundance and sex ratio in dioecious helminth parasites. Oecologia 111, 375-380.

parasites. Oecologia 111, 375-380.
Poulin, R., 2007. Are there general laws in parasite ecology? Parasitology 134, 763776.

Rieu, E., Recca, A., Bénet, J.j., Saana, M., Dorchies, P., Guillot, J., 2007. Reliability of coprological diagnosis of Paramphistomum sp. infection in cows. Veterinary Parasitology 146, 249-253.

Roberts, J.L., Swan, R.A., 1981. Quantitative studies of ovine haemonchosis. I. Relationship between faecal egg counts and total worm counts. Veterinary Parasitology 8, 165-171.

Roepstorff, A., BjØrn, H., Nansen, P., Barnes, E.H., Christensen, C.M., 1996 Experimental Oesophagostomum dentatum infections in the pig: worm populations resulting from trickle infections with three dose levels of larvae. International Journal for Parasitology 26, 399-408.

Rózsa, L., Reiczigel, J., Majoros, G., 2000. Quantifying parasites in samples of hosts. Journal of Parasitology 86, 228-232.

Seivwright, L.J., Redpath, S.M., Mougeot, F., Watt, L., Hudson, P.J., 2004. Faecal egg counts provide a reliable measure of Trichostrongylus tenuis intensities in freeliving red grouse Lagopus lagopus scoticus. Journal of Helminthology 78, 69-76. Sinniah, B., 1982. Daily egg production of Ascaris lumbricoides: the distribution of eggs in the faeces and the variability of egg counts. Parasitology 84, 167-175. Sinnialh, B., Sinniah, D., Subramaniam, K., 1981. Application of a 'standardized factor' to egg counting techniques for better prediction of worm burdens. Journal of Helminthology 55, 279-285

Sithithaworn, P., Tesana, S., Pipitgool, V., Kaewkes, S., Pairojkul, C., Sripa, B. Paupairoj, A., Thaiklar, K., 1991. Relationship between faecal egg count and worm burden of Opisthorchis viverrini in human autopsy cases. Parasitology 102 277-281.

Stringer, A.P., unpublished data. Investigating the parasites of black rhinoceros (Diceros bicornis). PhD Thesis. Centre for Biodiversity and Restoration Ecology, Victoria University of Wellington, Wellington, New Zealand.

Torgerson, P.R., Paul, M., Lewis, F.I., 2012. The contribution of simple random sampling to observed variations in faecal egg counts. Veterinary Parasitology 188, 397-401.

Zajac, A.M., Conboy, G.A., 2006. Veterinary Clinical Parasitology, seventh ed. Blackwell Publishing, Oxford. 



\title{
Appendix 3:
}

Oecologia

DOI $10.1007 / \mathrm{s} 00442-015-3319-1$

COMMUNITY ECOLOGY - ORIGINAL RESEARCH

\section{Host density drives macroparasite abundance across populations of a critically endangered megaherbivore}

\author{
A. P. Stringer ${ }^{1} \odot$ - W. L. Linklater ${ }^{1,2}$
}

Received: 9 May 2014 / Accepted: 10 April 2015

(c) Springer-Verlag Berlin Heidelberg 2015

\begin{abstract}
What determines the abundance of parasites is a central question within epidemiology. Epidemiological models predict that density-dependent transmission has a principal influence on parasite abundance. However, this mechanism is seldom tested in macroparasites, perhaps because multiple, comparable populations of the same host-parasite relationship are rare. We test the influence of a range of factors on parasite abundance across 18 populations of black rhinoceros (Diceros bicornis) in South Africa. Here we show that host density strongly predicts parasite abundance at the population level for both directly and indirectly transmitted parasites. All other models were not supported. The surprising influence of a single key factor, host density, within a complex ecological system demonstrates the validity of simple epidemiological models. Establishing this previously assumed relationship between host density and parasite abundance has major implications for disease control and parasite ecology. For instance, it is central to the idea of population density thresholds for parasitism, below which a parasite would become extinct. Density-dependent transmission is also essential for calculations of the basic reproductive number, and the hypothesis that parasites may regulate host population size.
\end{abstract}

Communicated by Jörg U. Ganzhorn.

\begin{tabular}{l}
\hline $\begin{array}{l}\text { A.P. Stringer } \\
\text { stringea@tcd.ie }\end{array}$ \\
। $\begin{array}{l}\text { Centre for Biodiversity and Restoration Ecology, Victoria } \\
\text { University of Wellington, Wellington, New Zealand }\end{array}$ \\
$2 \quad \begin{array}{l}\text { Centre for African Conservation Ecology, Nelson Mandela } \\
\text { Metropolitan University, Port Elizabeth, South Africa }\end{array}$
\end{tabular}

Published online: 24 April 2015
Keywords Anoplocephala gigantea $\cdot$ Strongyle nematodes · Disease transmission · Epidemiology $\cdot$ Diceros bicornis

\section{Introduction}

Epidemiological theory for parasite and disease abundance relies on the assumption of density-dependent transmission (Anderson and May 1991). High host density is assumed to increase parasite abundance because parasites more easily find a host to invade. Although predicted by numerous epidemiological models (Anderson and May 1979, 1991; May and Anderson 1979; Arneberg et al. 1998) and previously shown for microparasites (Lloyd-Smith et al. 2005), density-dependent transmission has rarely been documented for macroparasites of wildlife. This may be because macroparasite transmission is often more complicated than microparasite transmission due to external free-living life stages, which obscure the mechanism and have led to a number of alternative competing theories (Hudson and Dobson 1995). Also, for indirectly transmitted parasites, the mechanism is further complicated by the interaction between primary and secondary host density (Stien et al. 2010).

Density-dependent transmission is difficult to test because multiple, isolated, suitably variable, yet comparable populations of the same host-parasite interaction are rarely found. Such real-world experimental field data are needed to further develop epidemiological theory (Diamond 1986; Anderson and May 1991; Lloyd-Smith et al. 2005)

Arneberg et al. (1998) showed a positive relationship between the average abundance of parasites in different host species and host species density. However, the demonstration of an inter-specific association is not proof of a 
population-level ecological mechanism. Variability in host and parasite density through time for single host populations has been used to test the mechanism. A number of studies have shown a general positive relationship (Hudson et al. 1992; Albon et al. 2002; Jansen et al. 2012); however, negative relationships have also been reported (Haukisalmi and Henttonen 1990). It seems difficult to attribute cause where only single populations are used, but multiple influences are considered (Haukisalmi and Henttonen 1990; Cattadori et al. 2005). Here we test the influence of host density on parasite abundance by comparing multiple populations of the same host-parasite interaction.

Numerous other mechanisms may act on parasite abundance. For instance, environmental variables may impact the development rate or survival of external free-living parasite stages (Mouritsen and Poulin 2002), or for indirectly transmitted parasites, egg survival (Atkinson et al. 2013). Hence, we also test for the influence of a variety of environmental factors on parasite abundance as predicted by the literature. Likewise, the use of fire, as a form of intermediate disturbance, is likely to have a profound effect on the survival of eggs, free-living stages, and the abundance of intermediate hosts (Fuentes et al. 2007). Also, the size of a founder population may impact on the genetic diversity of either host or parasite population, potentially affecting the host-parasite interaction. The influence of fire regime and founder population size is also tested here.

Black rhinoceros (Diceros bicornis) are host to numerous parasites including a wide variety of both directly and indirectly transmitted gastrointestinal helminths (Penzhorn et al. 1994). Black rhino populations in South Africa are managed as an artificial meta-population. Between 1960 and 2005, in eastern South Africa, 442 rhino were reintroduced to reserves to speed species recovery. Remarkably, 17 out of the 18 populations of black rhinoceros in this study originated from only two, genetically very similar (Anderson-Lederer et al. 2012), source populations. Many are still expanding, providing a range of rhino densities that are not determined by their environment. We are able, therefore, to use these populations as different treatments in a fortuitous country-wide field or pseudoexperiment (Diamond 1986; Kerr et al. 2007). By comparing the abundance of both directly and indirectly transmitted parasites between these different populations, we can test for the influence of a range of environmental and ecological factors

\section{Materials and methods}

Fresh faecal samples ( $n=160$, mean samples per population $=8.9$, range $=3-18 ;$ Fig. 2) from 18 black rhino populations spanning a variety of climatic zones (Table 1) in
Table 1 Descriptive statistics from the environmental parameters used in candidate models

\begin{tabular}{lrlrr}
\hline & Mean & SE & Min. & Max. \\
\hline Max. temperature $^{\mathrm{a}}\left({ }^{\circ} \mathrm{C}\right)$ & 26.8 & 0.12 & 22.6 & 29.7 \\
Summer mean max. temperature $^{\mathrm{b}}\left({ }^{\circ} \mathrm{C}\right)$ & 28.8 & 0.14 & 23.8 & 32.4 \\
January min. temperature $^{\mathrm{b}}\left({ }^{\circ} \mathrm{C}\right)$ & 19.1 & 0.12 & 15.4 & 22.2 \\
Min. temperature $^{\mathrm{a}}\left({ }^{\circ} \mathrm{C}\right)$ & 13.8 & 0.14 & 10.1 & 17.0 \\
Humidity $^{\mathrm{a}}(\%)$ & 76.9 & 0.28 & 68.6 & 86.4 \\
Rainfall $\left(\right.$ mean monthly $^{\mathrm{a}}(\mathrm{mm})$ & 45.9 & 0.81 & 31.0 & 79.6 \\
Summer rainfall $^{\mathrm{b}}(\mathrm{mm})$ & 441.5 & 9.3 & 223.8 & 837.2
\end{tabular}

Summer rainfall ${ }^{b}(\mathrm{~mm})$

Max. Maximum, Min. minimum

${ }^{a}$ Mean value $2000-2011$

${ }^{b}$ During season of collection, summer: October-March

eastern South Africa were collected. The populations studied were all fenced and intensively monitored, hence rhino density could be accurately estimated. Population size ranged between nine and 200 individuals, within reserves that ranged from 3668 ha to 90,000 ha in size. Populations varied greatly in age. All populations had been created at least 3 years prior to data collection, with a mean age of 16.5 years (range 3-46 years) excluding the two source reserves. The majority of the study populations were established from only two source populations from South Africa (which were also included in the study), the solitary exception was created using a Namibian source population.

To mitigate the effects of season all samples were collected during the wetter summer season (October-April). The date of sample collection was originally included in the competing set of models and found to have no effect on parasite abundance. Fresh faeces were located along roads and game trails in the early morning (dawn-10 a.m.). To reduce the possibility of pseudoreplication a stratified random sampling regime, modified to ensure a minimum of $1 \mathrm{~km}$ between sample sites, was used. Also, local knowledge of rhino home ranges was used to reject samples that were likely to have come from the same individual.

Faecal samples were stored in anaerobic conditions in a cool box before immediate (ca. 2-4 h) analysis, or refrigerated at $4{ }^{\circ} \mathrm{C}$ for no more than $48 \mathrm{~h}$ before analysis. Faecal egg counts were performed using a modified McMaster technique, using Sheather's sugar solution (Zajac and Conboy 2006). The quality of samples was ensured by the identification of mature or immature embryonated eggs. Samples deemed too old based on egg maturation and field observation of faeces were rejected (Stringer et al. 2014). Eggs were identified using Zajac and Conboy (2006). Representative photos of all egg types found in a population were taken to confirm identification across populations. Two parasite groups were studied-strongyle-type nematodes (species of strongyle cannot be distinguished by egg

\section{包 Springer}


Appendix 3

Author's personal copy

Table 2 Information-theoretic table of candidate models explaining strongyle parasite abundance as a function of host rhinoceros density (rhino ha ${ }^{-2}$ )

\begin{tabular}{|c|c|c|c|c|c|}
\hline Model/hypothesis & Source & $K$ & $\mathrm{AlC}_{\mathrm{c}}$ & $\Delta \mathrm{AIC}_{\mathrm{c}}$ & $\omega$ \\
\hline Host density & Arneberg et al. (1998) & 3 & 2696.305 & 0.00 & 1.000 \\
\hline Max. temperature ${ }^{b}$ & Mouritsen and Poulin (2002) & 3 & 2716.509 & 20.20 & 0.000 \\
\hline January min. temperature ${ }^{c}$ & Hudson et al. (1992) & 3 & 2717.377 & 21.07 & 0.000 \\
\hline Summer mean max. temperature ${ }^{c}$ & Haukisalmi and Henttonen (1990) & 3 & 2717.540 & 21.24 & 0.000 \\
\hline Min. temperature ${ }^{\mathrm{b}}$ & Mouritsen and Poulin (2002) & 3 & 2718.423 & 22.12 & 0.000 \\
\hline Humidity ${ }^{\mathrm{b}}$ & Mouritsen and Poulin (2002) & 3 & 2720.264 & 23.96 & 0.000 \\
\hline Fire regime & Fuentes et al. (2007) & 3 & 2721.427 & 25.12 & 0.000 \\
\hline Population age & & 3 & 2721.172 & 25.87 & 0.000 \\
\hline Rainfall $^{\mathrm{b}}$ & Mouritsen and Poulin (2002) & 3 & 2722.378 & 26.07 & 0.000 \\
\hline Founder population size & Paterson et al. (1998) & 3 & 2724.379 & 28.07 & 0.000 \\
\hline Summer rainfall $^{c}$ & Haukisalmi and Henttonen (1990) & 3 & 2727.266 & 30.96 & 0.000 \\
\hline Base model ${ }^{\mathrm{d}}$ & & 2 & 2730.649 & 34.34 & 0.000 \\
\hline
\end{tabular}

Models are in descending order from most to least supported based on Akaike second-order information criteria $\left(A I C_{c}\right)$. The set of candidate models differs between parasite groups as they use different transmission methods. For other abbreviations, see Table 1

$K$ Number of parameters in a model

"The confidence set of models, i.e. $>95 \%$ of Akaike weights $(\omega)$

${ }^{b}$ Mean value 2000-2011s

c During season of collection

d The base model, which included only the random effect for population

morphology alone) and Anoplocephala sp. In South Africa, seven species of strongyle have been identified that infect black rhino, while only one cestode has been identified, Anoplocephala gigantea. All of these are thought to be host specific to black rhino (Penzhorn et al. 1994; Stringer 2015). Strongyle nematodes are directly transmitted parasites-eggs develop into free-living stages which crawl onto vegetation and are subsequently eaten. A. gigantea is an indirectly transmitted tapeworm-eggs are eaten by an oribatid mite (Oribatida), which are subsequently thought to crawl onto vegetation before being accidentally eaten (Zajac and Conboy 2006).

To measure parasite abundance ideally autopsies would be performed on fresh carcasses. As we were working with a long-lived and critically endangered host species this was not possible. Faecal egg counts have previously been shown in other odd-toed ungulates and with similar parasite groups to be a broadly reliable indicator of parasite abundance (Nielsen et al. 2010; Stringer et al. 2014). For instance, Kjaer et al. (2007) found a positive correlation of 0.71 between worm burden and faecal egg count for the tapeworm Anoplocephala perfoliata in domestic horses (Equus ferus caballus). Interestingly, the high abundance of eggs within faecal samples found in black rhino may increase the accuracy of faecal egg counts (Denwood et al. 2012).

Here we use the term 'abundance' as described by Bush et al. (1997), in that we calculated an estimated intensity of infection within each host, but also included non-infected hosts in our analysis. Hence, our results may reflect both the prevalence and the intensity of infection within a population.

An information-theoretic approach was used to explain parasite abundance by comparing candidate models. The competing candidate set of models (hypotheses) was developed by consulting the literature (Tables 2, 3). Sample size for some populations was very small. Hence we used mixed models to test the relationship of candidate models to parasite abundance, and either generalised linear mixed models (GLMM) or linear mixed models (LMM) were used (see "Results"). Population could then be specified as a random effect, allowing us to utilise all samples collected, rather than reducing each population to a single mean value. Furthermore, a second-order Akaike information criterion $\left(\mathrm{AIC}_{\mathrm{c}}\right)$ was used to compare competing models, which takes into account sample size. For GLMMs, a negative binomial distribution with a log link was used. In all models the parameters were the same except for the fixed effect. $K$ relates to the number of parameters in each model (i.e. $K=3=$ fixed effect + random effect + intercept).

Models with the lowest $\mathrm{AIC}_{c}$ were deemed to have the strongest support. Model selection was based on the top performing models that represented $95 \%$ of Akaike weights $(\omega)$. A base model containing only the random effect was included in the competing set of models to investigate how much information in the data was not 
Appendix 3

Author's personal copy

Oecologia

Table 3 Information-theoretic table of candidate models explaining Anoplocephala gigantea abundance as a function of host rhinoceros density $\left(\right.$ rhino ha ${ }^{-2}$ )

\begin{tabular}{llllll}
\hline Model/hypothesis & Source & $K$ & $\mathrm{AlC}_{\mathrm{c}}$ & \multicolumn{1}{c}{$\Delta \mathrm{AlC}_{\mathrm{c}}$} & $\omega$ \\
\hline Host density $^{\mathrm{a}}$ & Arneberg et al. (1998) & 3 & 507.486 & 0.00 & 0.930 \\
Mean max. summer temperature $^{\mathrm{a}, \mathrm{b}}$ & Haukisalmi and Henttonen (1990) & 3 & 514.123 & 6.64 & 0.034 \\
Max. temperature $^{\mathrm{c}}$ & Atkinson et al. (2013) & 3 & 515.084 & 7.60 & 0.021 \\
Min. temperature $^{\mathrm{c}}$ & Atkinson et al. (2013) & 3 & 517.383 & 9.90 & 0.007 \\
Base model $^{\mathrm{d}}$ & & 2 & 517.427 & 9.94 & 0.006 \\
Humidity $^{\mathrm{c}}$ & Atkinson ct al. (2013) & 3 & 519.885 & 12.40 & 0.002 \\
Fire regime $_{\text {Rainfall }}$ & Fuentes et al. (2007) & 3 & 522.491 & 15.01 & 0.001 \\
Population age $_{\text {Founder population size }}$ & Atkinson et al. (2013) & 3 & 523.000 & 15.51 & 0.000 \\
Summer rainfall $^{\mathrm{b}}$ & & 3 & 526.305 & 18.82 & 0.000 \\
& Paterson et al. (1998) & 3 & 527.218 & 19.73 & 0.000 \\
\hline
\end{tabular}

Models are in descending order from most to least supported based on $\mathrm{AIC}_{\mathrm{c}}$. The set of candidate models differs between parasite groups as they use different transmission methods. For abbreviations, see Tables 1 and 2

${ }^{a}$ The confidence set of models, i.e. $>95 \%$ of $\omega$

${ }^{b}$ During season of collection

c Mean value $2000-2011$

d The base model, which included only the random-effect for population

being explained by competing models. To avoid overfitting (Zuur et al. 2009), within each model $K$ was kept to a minimum by initially including only one fixed effect per model (excluding the base model which had no fixed effects). A suite of models with two fixed effects was then produced that contained the top performing predictor variable plus each of the other parameters in turn. As full models could not be used the information-theoretic approach could not be utilised fully in this study. However, we view this study as providing a useful framework which can be improved upon, either with further populations of black rhino, or with species that are not as rare as black rhino.

SPSS (IBM 2011) was used for all statistical calculations. Fire regime was estimated based on reserve records. Environmental variables were obtained by the South African Weather Service station closest to the reserve (mean distance $27.6 \mathrm{~km}$ ) monthly, and varied extensively between reserves (Table 1)

\section{Results}

The directly transmitted strongyle-type nematodes were found in all populations except one, at a mean population abundance of 1918 eggs g $^{-1}$ (e.p.g.) (range $44-4075$ e.p.g. SD = 1438). The indirectly transmitted cestode, $A$. gigantea, was found in all populations at a mean population abundance of 228 e.p.g. (range 33-546 e.p.g., SD = 149).

Model fit when analysing the data utilising a GLMM with a negative binomial distribution and a log link for the strongyle parasite group was poor. Further investigation revealed that the data did not follow a negative binomial distribution; skewness was low (0.5), with the mean egg count (2280 e.p.g.) close to the median (2150 e.p.g.). Also, kurtosis was very low $(-0.5)$ indicating a wide flat peak to the data, which was likely to be the reason why the data were considered non-normal based on the Kolmogorov-Smirnov test (statistic $=0.98, p=0.001$ ). This distribution is likely a result of combining data from multiple populations. While each individual population has an aggregated parasite distribution, when combined, different levels of the distribution peak for each population will create multiple peaks. This ultimately results in a single wide flat peak, which when viewed in a p-p plot does not seem to differ meaningfully from normality. This does not occur for A. gigantea, as egg counts for the different populations were much less variable. The competing set of models taken from single-factor model tables did not differ between using a GLMM and a LMM on untransformed data for the strongyle group. Hence, results from the LMM are presented and plotted (Fig. 1). While using a GLMM, no two-factor model improved on the top model. However, when using a LMM all two-factor models containing the top model improved on the original model, while all threefactor models including the top two models improved on all two-factors models. This is a clear sign of overparameterization, hence only one-factor models for the strongyle group are presented.

Using multi-model selection and inference to explain parasite abundance, host density models performed best for

\section{包 Springer}




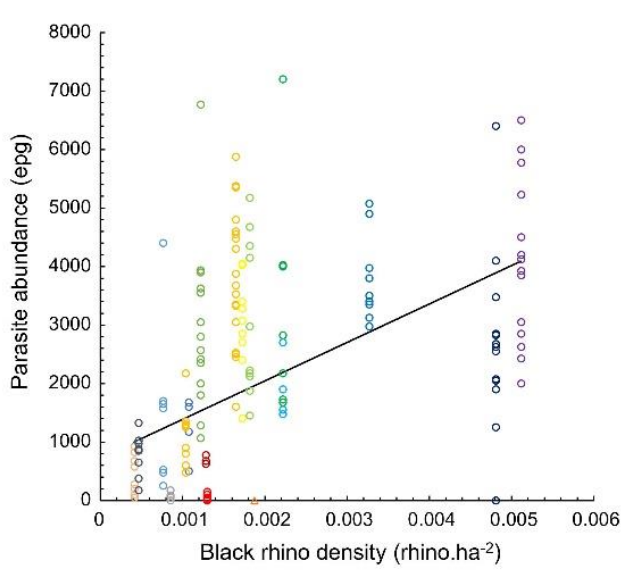

Fig. 1 Scatter plot showing strongyle abundance in host population against the density of black rhino (rhino ha ${ }^{-2}$ ) in that population. Open circles represent individual samples $(n=160)$, and the colour of the circle (available in the online version only) can be used to differentiate between different populations. The triangle represents al samples that came from the single population that was sourced from a Namibian source population. The trend line represents the fitted line using parameters from our results (linear mixed models)

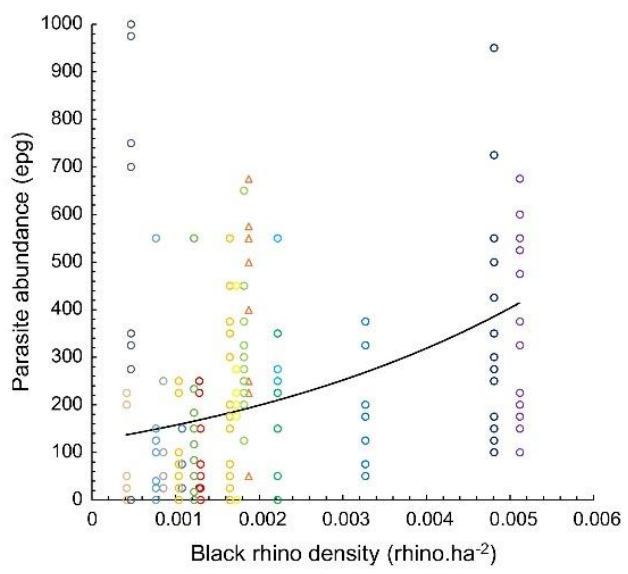

Fig. 2 Scatter plot showing Anoplocephala gigantea abundance in host populations against the density of black rhino (rhino ha-2) in that population. Open circles represent individual samples $(n=160)$, and the colour of the circle (available in the online version only) can be used to differentiate between different populations. Triangles represent individual samples that came from the single population that was sourced from a Namibian source population. Two data point are not included in this graphic for ease of display [2516 eggs $\mathrm{g}^{-1}$ (e.p.g.) $\times 0.0012$ rhino ha ${ }^{-2}, 1875$ e.p.g. $\times 0.0051$ rhino ha $a^{-2}$. The (generalised linear mixed models) the directly transmitted, strongyle parasite group (Table 2; Fig. 1; coefficient 657,404.5, SE 212,871, intercept 730.5, SE 485.9). Host density contributed $100 \%$ of $\omega$, while all other models were implausible, receiving no support. For the indirectly transmitted $A$. gigantea the host density model performed best, contributing $93 \%$ of $\omega$ (Table 3; Fig. 2; coefficient 235.4, SE 117.0, intercept 4.8, SE 0.3). Parasite abundance was again not explained by most environmental parameters, although mean maximum summer temperature was included in the confidence set of models $\left(\sum \omega>0.95\right)$ (Zuur et al. 2009).

Each predictor was added to host density to see if models with two fixed effects could improve on the host density model for A. gigantea. A model containing host density and mean maximum summer temperature was the only model to improve on the original host density model $\left(\Delta \mathrm{AIC}_{\mathrm{c}}=-0.35\right.$, maximum temperature coefficient -0.06 , SE 0.09 , host density coefficient 188.1, SE 142.4, intercept 7.1, SE 3.0). However, auto-correlation between the two fixed effects in the model means that estimates of these parameters are not reliable, and the model table comparing the single fixed effects (Table 3 ) should be utilised.

\section{Discussion}

For directly transmitted parasites, we conclude that host density is the only predictor in this study that influences population-level parasite abundance. This is despite numerous other hypotheses predicting that environmental factors, such as temperature, rainfall, and humidity, will influence larval survival and hence are of importance to parasite abundance (Haukisalmi and Henttonen 1990; Paterson et al. 1998; Mouritsen and Poulin 2002; Fuentes et al. 2007; Atkinson et al. 2013). The relationship between primary host density and parasite abundance for indirectly transmitted parasites is thought to be more complex due to the influence of secondary host density (Stien et al. 2010). Despite this we show a clear relationship between primary host density and parasite abundance for the indirectly transmitted A. gigantea (Fig. 2). Secondary host density does not mask the relationship between primary host density and parasite abundance for A. gigantea and its black rhino host, perhaps because of the ubiquity of the secondary host across the landscape and between different populations of black rhino (Van Nieuwenhuizen et al. 1994).

The host density model was marginally improved for $A$. gigantea by also including mean maximum summer temperature. This weak negative relationship between summer maximum temperature and parasite abundance has been found previously in other mammal-cestode relationships, and is likely due to egg desiccation reducing abundance in hotter areas (Haukisalmi and Henttonen 1990). 
Strongyle nematode eggs were found in all populations, except the single population that originated from a Namibian source population. The Namibian population's habitat and rainfall are very different from those of all directly studied populations, and it also has an extraordinarily low black rhino density (i.e. 0.00013 rhino $\mathrm{ha}^{-2}$ ). Hence, it is possible that strongyles became extinct or never existed in the source population, or were not translocated with the host when reintroduced to the South African reserve.

There may be other mechanisms, rather than densitydependent transmission, that cause host density to drive parasite abundance. An individual's parasite abundance has been linked to their age. If a population is founded with younger individuals, then, as that population ages and grows denser, the age-structure within the population may change. However, in this study the age of a population had no impact on parasite abundance.

There was little or no support for models including only the random effect compared to mixed-effect models-indicating that the fixed effects in the supported models had substantial value in explaining variation in the response variable (parasite burden). The leading mixed-effect models might still be improved in the future by the addition of other fixed effects not tested here. These mechanisms may include host susceptibility, where inbred hosts may be more susceptible to parasite invasion, and within-host parasite community interactions, where competition and facilitation between parasite species may limit or increase parasite abundance (Pedersen and Fenton 2007). For instance, it has been shown that host susceptibility may affect variation in parasite abundance between individuals (Paterson et al. 1998; Poulin 2007).

Black rhino are a critically endangered and elusive species which meant that samples were not readily available, and sample size for some populations was very low. However, black rhino may be an excellent model organism for testing these hypotheses, due to their solitary nature (Altizer et al. 2003), the slow rate of any natural changes in population size, and the host species specificity of the parasites studied. We also used modern statistical techniques that meant populations did not have to be reduced to a single statistical average (mixed models). Furthermore, by utilising the fortuitous experiment created by the translocation and reintroduction of black rhino in South Africa we were able to compare multiple populations of the same host-parasite interaction, a novel method for investigating epidemiological hypotheses. Hence, despite the restrictions of small sample size, and using faecal egg counts rather than directly measuring abundance, we were still able to show that host density is a singularly powerful predictor of macroparasite abundance for a directly transmitted parasite, and a highly influential predictor within an indirectly transmitted parasite.
Our demonstration of a previously assumed mechanism for parasite abundance gives real-world evidential support to epidemiological theory. This may have a wide range of implications for parasite control (Gortázar et al. 2006), disease prevention (Anderson and May 1991), and conservation (Woodroffe 1999). The assumption of densitydependent transmission is often used for determining the basic reproductive number (Anderson and May 1991) the expected number of secondary cases arising from one infected individual, and hence underpins much of current epidemiology. It also underpins some key questions in parasitology, such as whether sub-clinical parasitism can regulate host population size (Hudson et al. 1992). The dominance of host density in explaining population-level parasite abundance also supports the expectation of a population density threshold (Lloyd-Smith et al. 2005), below which a parasite will become extinct. This paper, then, gives support to many current parasite- and disease-control programs that work to reduce the density of susceptible individuals. As susceptible host density is a determinant of parasite abundance, reducing susceptible host density by rising vaccination rates should increase the likelihood of local parasite extirpation.

Author contribution statement A. P. S. and W. L. L. conceived and designed the study. A. P. S. performed the study and analysed the data. A. P. S. wrote the manuscript with important input and editorial advice from W. L. L.

Acknowledgments Special thanks go to Eastern Cape Parks and Acknowledgments Special thanks go to Eastern Cape Parks and
Tourism Agency and associated staff. Dr. Dean Peinke, Ezemvelo Tourism Agency and associated staff, Dr. Dean Peinke, Ezemvelo
KwaZulu-Natal (KZN) Wildlife and associated staff, Chris Kelly, NelKwaZulu-Natal (KZN) Wildlife and associated staff, Chris Kelly, Nel-
son Mandela Metropolitan University, Prof Graham Kerley, Dr. Shirley Parker-Nance, Victoria University of Wellington, Prof. Graham Le Gros, Dr. Roan Plotz, Dr. Rosalyn Anderson-Lederer, the World Wide Fund for Nature, Dr. Jacques Flamand, Dr. James Martin, Megan Towers, the South African Weather Service, and the owners, staff and black rhino monitors from numerous private reserves for their assistance. This project was made possible by funds from the US Fish and Wildlife Service (grant numbers 98210-6-G102, 98210-4-G920), and a Victoria University of Wellington Doctoral Assistantship.

\section{References}

Albon SD, Stien A, Irvine RJ, Langvatn R, Ropstad E, Halvorsen O (2002) The role of parasites in the dynamics of a reindeer population. Proc R Soc B Biol Sci 269:1625-1632

Altizer S, Nunn CL, Thrall PH, Gittleman JL, Antonovics J, Cunningham AA, Dobson AP, Ezenwa V, Jones KE, Pedersen AB, Poss M, Pulliam JRC (2003) Social organization and parasite risk in mammals: integrating theory and empirical studies. Annu Rev mammals: integrating theor

Anderson RM, May RM (1979) Population biology of infectious diseases. Part I. Nature 280:361-367

Anderson MR, May RM (1991) Infectious diseases of humans: dynamics and control. Oxford University Press, New York 
Anderson-Lederer RM, Linklater WL, Ritchie PA (2012) Limited mitochondrial DNA variation within South Africa's black rhino (Diceros bicornis minor) population and implications for management. Afr J Ecol 50:404-413

Arneberg P, Skorping A, Grenfell B, Read AF (1998) Host densities as determinants of abundance in parasite communities. Proc $\mathrm{R}$ Soc B Biol Sci 265:1283-1289

Atkinson JAM, Gray DJ, Clements AC, Barnes TS, McManus DP, Yang YR (2013) Environmental changes impacting Echinococcus transmission: research to support predictive surveillance and control. Glob Change Biol 19:677-688

Burnham K, Anderson D (2002) Section 6.6. Random co-efficient models. Model selection and multimodel inference: a practical information-theoretic approach, 2nd edn. Springer, New York

Bush AO, Lafferty KD, Lotz JM, Shostak AW (1997) Parasitology meets ecology on its own terms: Margolis et al. revisited. J Parasitol 83:575-583

Cattadori IM, Haydon DT, Hudson PJ (2005) Parasites and climate synchronize red grouse populations. Nature 433:737-741

Denwood MJ, Love S, Innocent GT, Matthews L, McKendrick IJ, Hillary N, Smith A, Reid SWJ (2012) Quantifying the sources of variability in equine faccal egg counts: implications for improving the utility of the method. Vet Parasitol 188:120-126

Diamond J (1986) Overview: laboratory experiments, field experiments, and natural experiments. In: Diamond J, Case T (eds) Community ecology. Harper and Row, New York

Fuentes MV, Sainz-Elipe S, Galán-Puchades MT (2007) Ecological study of the wood mouse helminth community in a burned Mediterranean ecosystem in regeneration five years after a wildfire. Acta Parasitol 52:403-413

Gortázar C, Acevedo P, Ruiz-Fons F, Vicente J (2006) Disease risk and overabundance of game species. Eur J Wildl Res 52:81-87

Haukisalmi V, Henttonen $\mathrm{H}$ (1990) The impact of climatic factors and host density on the long-term population dynamics of vole helminths. Oecologia 83.309-315

Hudson PJ, Dobson AP (1995) Macroparasites: observed patterns in naturally fluctuating animal populations. In: Grenfell BT, Dobson AP (eds) Ecology of infectious diseases in natural populations. Cambridge University Press, Cambridge

Hudson PJ, Newborn D, Dobson AP (1992) Regulation and stability of a free-living host-parasite system: Trichostrongylus tenuis in red grouse. I. Monitoring and parasite reduction experiments. J Anim Ecol 61:477-486

IBM (2011) SPSS statistics version 20

Jansen PA, Kristoffersen AB, Viljugrein H, Jimene $z$ D, Aldrin M Stien A (2012) Sea lice as a density-dependent constraint to salmonid farming. Proc R Soc B Biol Sci 279:2330-2338. doi:10.1098/rspb.2012.0084

Kerr JT, Kharouba HM, Currie DJ (2007) The macroecological contribution to global change solutions. Science 316:1581-1584. doi: $10.1126 /$ science. 1133267
Kjaer LN, Lungholt MM, Nielsen MK, Olsen SN, Maddox-Hyttel C (2007) Interpretation of serum antibody response to Anoplocephala perfoliata in relation to parasite burden and faecal egg count. Equine Vet J 39:529-533

Lloyd-Smith JO, Cross PC, Briggs CJ, Daugherty M, Getz WM, Latto J, Sanchez MS, Smith AB, Swei A (2005) Should we expect population thresholds for wildlife disease? Trends Ecol Evol 20:511-519

May RM, Anderson RM (1979) Population biology of infectious diseases. II. Nature 280:455-461

Mouritsen KN, Poulin R (2002) Parasitism, climate oscillations and the structure of natural communities. Oikos 97:462-468

Nielsen MK, Vidyashankar AN, Andersen UV, DeLisi K, Pilegaard K, Kaplan RM (2010) Effects of fecal collection and storage factors on strongylid egg counts in horses. Vet Parasitol 167:55-61

Paterson S, Wilson K, Pemberton JM (1998) Major histocompatibility complex variation associated with juvenile survival and parasite resistance in a large unmanaged ungulate population (Ovis aries L.). Proc Natl Acad Sci USA 95:3714-3719

Pedersen AB, Fenton A (2007) Emphasizing the ecology in parasite community ecology. Trends Ecol Evol 22:133-139

Penzhorn BL, Krecek RC, Horak IG, Verster AJM, Walker JB, Boomker JDF, Knapp SE, Quandt SKF (1994) Parasites of African rhinos: a documentation, pp 168-175, tables 1-2. In: Penchorn BL, et al (eds) Proceedings of a Symposium on Rhinos as Game Ranch Animals, Onderstepoort, Republic of South Africa, 9-10 September 1994, pp i-iv, 1-242

Poulin R (2007) Evolutionary ecology of parasites, 2nd edn. Princeton University Press, Princeton

Stien A, Voutilainen L, Haukisalmi V, Fuglei E, Mørk T, Yoccoz NG, Ims RA, Henttonen H (2010) Intestinal parasites of the Arctic fox in relation to the abundance and distribution of intermediate hosts. Parasitology 137:149-157

Stringer AP (2015) Parasite ecology and the conservation biology of black rhinoceros (Diceros bicornis). Unpublished $\mathrm{PhD}$ dissertation, Victoria University of Wellington, Wellington

Stringer AP, Smith D, Kerley GIH, Linklater WL (2014) Reducing sampling error in faecal egg counts from black rhinoceros (Diceros bicomis). Int J Parasitol Parasites Wildl 3:1-5. doi:10.1016/j.ijppaw.2013.10.002

Van Nieuwenhuizen LC, Verster AJM, Horak IG, Krecek RC, Grimbeck JR (1994) The seasonal abundance of oribatid mites (Acari: Cryptostigmata) on an irrigated Kikuyu grass pasture. Exp Appl Acarol 18:73-86

Woodroffe R (1999) Managing disease threats to wild mammals. Anim Conserv 2:185-193

Zajac AM, Conboy GA (2006) Veterinary clinical parasitology, 7th edn. Blackwell, Oxford

Zuur AF, Ieno EN, Walker NJ, Saveliev AA, Smith GM (2009) Mixed effects models and extensions in ecology with R. Springer, Berlin 



\section{Appendix 4:}

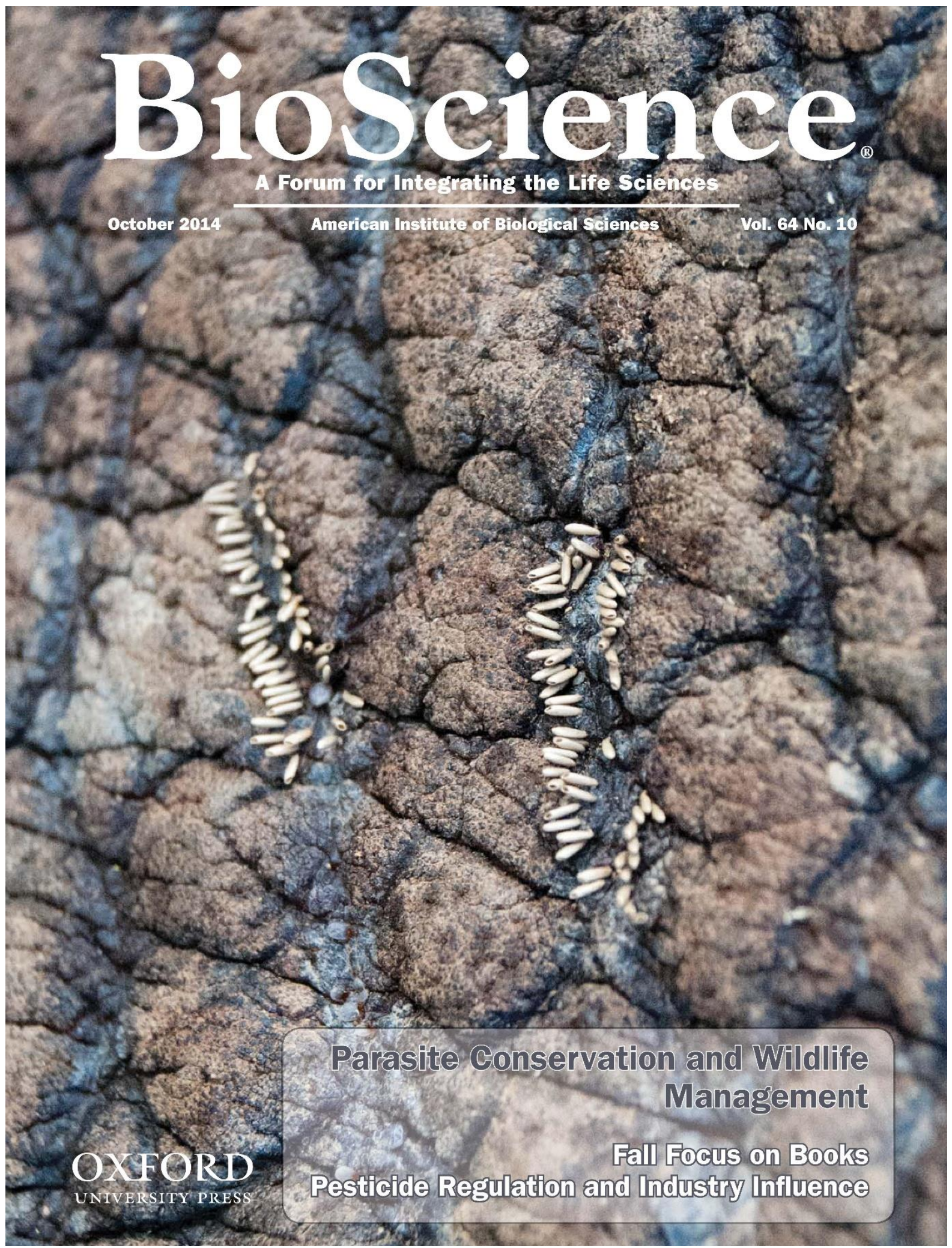


Cover: Eggs from the largest fly in Africa, the rhinoceros bot fly, Gyrostigma rhinocerontis, are clearly visible on the skin of a black rhinoceros (Diceros bicornis) in South Africa. The fly's larvae hatch and migrate to the stomach, where they attach to the stomach wall. When mature, they are ejected within feces and pupate into nonfeeding adults that survive for just a few days. The photograph was taken during the translocation of black rhinoceros for conservation and range expansion. Translocations may act as significant parasite bottlenecks, restricting parasite recolonization within newly created host populations. In their article, Andrew Stringer and Wayne Linklater discuss the importance of conserving parasites, which may in some cases justify establishing refugia. Photograph: Andrew Stringer. 


\title{
Everything in Moderation: Principles of Parasite Control for Wildife Conservation
}

\author{
ANDREW PAUL STRINGER AND WAYNE LINKLATER
}

Parasites can reduce host body condition, impair reproduction, and cause mortality. However, parasites are a major source of biodiversity, are a fundamental component of a healthy ecosystem, and could be the group most affected by the modern-day biodiversity crisis. Parasite control a fundamental component of a healthy ecosystem, and could be the group most affected by the modern-day biodiversity crists. Parasite control
may cause immunological naivety, unbalance parasite-mediated apparent competition between hosts, and destabilize the host-parasite arms may cause immunological naivety, unbalance parasite-mediated apparent competition between hosts, and destabilize the host-parasite arms
race. Here, we rank parasite control strategies according to their potential impact on ecosystems. We argue that, as the threat that a parasite race. Here, we rank parasite control strategies according to thetr potential impact on ecosystems. We argue that, as the threat that a parasite
poses to host survival increases, the need for parasite control increases, and, therefore, control measures that have a greater impact on ecosystems poses to host survival increases, the need for parasite control increases, and, therefore, control measures that have a greater impact on ecosystems
can be justified. However, because host-specific parasites may be more endangered than their hosts, there is often the need for active parasite can be justified. However, because host-specific parasites may be more endangered than their hosts, there is often the need for active parasite conservation strategies such as establishing parasite refugia. Although the principles proposed here are predominantly intuitive, there are
numerous examples in which they have not been applied.

Keywords: parasitology, conservation, biodiversity, applied ecology, endangered species

he coextinction of dependent species, such as mutualists, commensals, and parasites, may be the great est but least appreciated source of current biodiversity decline (Dunn et al. 2009). Parasites may be a particularly influential component of biodiversity, because they act as evolutionary drivers of traits such as host genetic and phenotypic diversity, secondary sexual traits, and community structure (Nunn et al. 2004, Gómez and Nichols 2013) However, parasites can also be a danger to threatened species because of their impacts on populations (Heard et al. 2013). Furthermore, host inbreeding, environmental pollution, overexploitation, habitat modification and loss, climate change, contact with domestic livestock, and invasive species may all increase the potential for parasites to do harm (Lafferty and Gerber 2002, Smith et al. 2009).

Conservation actions often do not take into account the importance of parasites. For instance, translocation programs often do not acknowledge the parasite bottleneck that they cause and that restricts parasite biodiversity within reintroduced populations (Moir et al. 2012). Indeed, gra wolves (Canis lupus) reintroduced to Yellowstone National Park were treated with a broad-spectrum antiparasitic before their release (Almberg et al. 2012). Active parasite conservation strategies are rare (Gómez and Nichols 2013), and conservation management in the past has led directly to parasite extinctions. For instance, black-footed ferrets (Mustelo nigripes) have a depauperate ectoparasite fauna thought to be caused by previous conservation actions (Harris et al 2014). The extinct California condor louse (Colpocephalum californici) was deliberately killed during the captive breeding of its host the Californian condor (Gymnogyps californianus; Koh et al. 2004).

Guidelines for the control of parasites in threatened hosts are limited or nonexistent (Woodroffe 1999). Wildlife parasite control programs have been both successful, which clearly reduces the impacts of epidemics (Knobel et al. 2008), and damaging, having detrimental unintended side effects on the hosts (Burrows et al. 1995). Here, we outline the arguments for and against parasite control. We then propose some first principles of parasite control in threatened host species in which we attempt to balance the value of parasite biodiversity and ecosystem roles with the need to protect species of conservation concern.

\section{Reasons for parasite control}

Parasites can cause severe population declines. For instance, between 1990 and 1992, the rabies virus was thought to be the cause of an approximately $69 \%$ decline in the Bale Mountain Ethiopian wolf (Canis simensis) population (Randall et al. 2004). Many parasites do not directly cause mortality but do impair body condition; reduce growth or fecundity; or exacerbate preexisting threats to hosts,

BioScience 64: 932-937. The Author(s) 2014. Published by Oxford University Press on behalf of the American Institute of Biological Sciences. All rights Bioscience 64: 932-937. The Author(s) 2014. Published by Oxford Unive
reserved. For Permissions, please e-mail: journals.permissions@oup.com. doi:10.1093/biosci/biul35

Advance Access publication 3 September 2014 
such as predation or winter survival (Irvine 2006). These effects affect a population's performance and can put that population at risk. For instance, the intestinal nematode Trichostrongylus tenuis is one of the causes of large population fluctuations in its host, the red grouse (Lagopus lagopus, Martinez-Padilla et al. 2014). The influences of parasitism on hosts may also be more cryptic. Schwanz (2008) showed that parasitized female deer mice (Peromyscus maniculatus) produced offspring that were larger in ways that improved the offspring's fitness but sacrificed the longer-term reproductive potential of the mother.

Threatened species are at particular risk from parasitism (Heard et al. 2013), perhaps because the cause of biodiversity loss amplify the parasite burden (Smith et al. 2009). Threatened species are also more at risk from inbreeding, which may reduce a host's immunological defenses and resistance (Cassinello et al. 2001). For instance, Acevedo-Whitehouse and colleagues (2003) showed that inbred Californian sea lions (Zalophus californianus) showed increased incidences of parasitism and that these individual may act as reservoirs for further infection.

Climate change is predicted to change the geographic range of both parasites and hosts, which may lead to novel species pairings (Altizer et al. 2013). This will increase the occurrence of emerging infectious diseases, which can be devastating. For instance, white-nose syndrome, caused by a psychrophilic fungus (Pseudogymnoascus destructans, formerly Geomyces destructans), has recently caused a population collapse of brown myotis bats (Myotis lucifugus) in North America, with a mean population decline of $73 \%$ (Frick et al. 2010). Therefore, because the problems associated with parasitism are expected to grow strategies to control parasitism will become of increasing importance.

\section{Detrimental impacts of parasite control}

Parasites are a fundamental component of a healthy ecosystem and a key link with food webs (Hudson et al. 2006); the removal of parasites can have numerous unintended consequences. Treatment of a human with antibiotics, for example, can eliminate naturally occurring competitors of the bacteria Clostridium difficile. This allows C. difficile to reproduce and spread much more widely in the intestine causing antibiotic-associated colitis (Johnson and Gerding 1998). The removal of an endophytic fungus from stout woodreed (Cinna arundinacea) increased host survival, but decreased host population growth because of the effects that the fungus has on plant regeneration (Rudgers et al. 2012).

Parasite control can also affect the resilience of a hos to parasitism. If a parasite is eradicated from a popula tion, future generations of the host species will not have immunological experience with that parasite. If the parasite is reintroduced to the population, naive individuals could be more vulnerable to it. For instance, translocations of naive black rhinoceros (Diceros bicornis) into trypanosome
(Trypanosoma spp.) endemic areas must be timed with seasonally low trypanosome infection rates to prevent the deaths of the translocated individuals (Mihok et al. 1995).

Parasite control can also affect competition between host species. Parasite-mediated apparent competition occurs when two host species are infected by the same parasite species but the cost of infection differs between the two hosts. For instance, it is predicted (Chauvenet et al. 2011) that the control of canine distemper virus in the Serenget would positively affect both lion (Panthera leo) and cheetah (Acinonyx jubatus) populations. However, the lion populations would receive the greater benefit, and denser lion populations would then be directly detrimental to the cheetah populations.

Controlling parasitism may fundamentally disrupt host evolution, because the benefits of control will be biased toward those individuals most detrimentally affected by parasites. According to the red-queen hypothesis, the theory that populations must be constantly evolving to remain competitive, the success of parasite-susceptible individuals will potentially create a future population less resistant to parasites (Lively and Dybdahl 2000). Finally, parasites are a major driving force behind evolution and speciation (Nunn et al. 2004). Therefore, removing parasites counters one of the overall aims of conservation-to sustain ecological processes, including adaptation and evolution.

The benefits of controlling parasites in threatened species, to improve fitness and population performance, then, must be balanced with the individual ethical and ecosystem costs associated with control. A scheme for targeted parasite control that is appropriate to the magnitude of the threat that a parasite poses, taking wider unintended impacts into account, and that incorporates the risks to parasite biodiversity, is clearly desirable.

\section{A proposal for the first principles of parasite control} in threatened species

Here, we present a proposal for the first principles of parasite control with threatened species:

Principle 1: The greatest need for parasite control is when a host is critically endangered and a parasite greatly affects population performance. This is because the need for parasite control increases as the threat that a parasite poses to the host-species' survival increases. This can be evaluated: As the extinction risk of the host (e.g., its International Union for Conservation of Nature [IUCN] Red List category) and the negative impact of the parasite on the host's population performance increase, so does the parasite threat. Therefore, if a parasite has little effect on population performance and the host is at low risk of extinction, there is little need to control the parasite. In a species of least concern there is no conservation-based argument for parasite control above that required for ecological restoration, unless the species is a reservoir host for parasites of a more endangered target species. 
Principle 2: As the need for parasite control increases, control strategies with greater impacts on parasite populations and with higher impacts on the ecosystem can be justified (see the "Types of parasite control" section below).

Principle 3: For host-specific parasites, as the risk of parasite extinction increases, so, too, do the importance of creating parasite refugia and the need for active parasite conservation increase. Threatened hosts will contain threatened parasites, which are likely to go extinct before the host goes extinct (Altizer et al. 2007). Indeed, a single parasite species may not be found throughout its host's geographic range and therefore, the parasite's extinction risk could far exceed its host's. Parasite control will further increase this extinction risk. If the long-term stability of the threatened host species is ensured, parasite refugia can be used to reintroduce lost species and to restore natural ecosystem functioning (Moi et al. 2012). Here, we assume the precautionary view that parasite host shifts are not linked to the scarcity of hosts (Colwell et al. 2012)

Principle 4: For generalist parasites, parasite control techniques that disrupt a host population's evolutionary response to parasitism should be avoided. This is because of the threat of reinfection: Subsequent reinfections may more detrimentally affect a genetically weakened population. For instance, strategies that increase host population vulnerability by interfering with the host-parasite arms race (such as vac cination and parasite treatment) should only be considered when there are so few hosts left that every individual is important for the survival of the species. Importantly, the control of generalist parasites in threatened hosts will have minimal impacts on the overall abundance of the parasite, because the parasite population will be maintained within other reservoir hosts.

\section{Types of parasite control}

To apply these proposed first principles, it is important to identify how different types of parasite control differ in their impacts on individuals and ecosystems. This will allow conservation managers to choose parasite control strategies that are proportionate to the threat that a parasite poses to its host's extinction. 'Therefore, in table 1, we attempt to broadly rank parasite control strategies by their potential detrimenta impacts on ecosystems and host populations.

The least damaging forms of parasite control are closest, in principle, to ecological restoration. For instance, reducing inbreeding depression aims to restore the ability of a host population to evolve in response to parasite pressure. Ecologically invasive strategies, in contrast, alter ecosystem functioning to decrease the parasite burden of a host population. For instance, a habitat may be modified to reduce the abundance of a parasite's vectors. The final grouping (evolutionarily disruptive strategies) contains strategies that are potentially the most damaging forms of parasite control.
These are ecologically invasive and, in particular, directly interfere with the host-parasite arms race. For instance, the vaccination of hosts will increase the reproductive success of parasite-susceptible hosts. This may lead to a generation's being more susceptible to parasitism, akin to breeding a generation of antelope that cannot outrun a common predator. Furthermore, vaccination may alter parasite evolution, which may lead to more-virulent strains of the parasite (Gandon et al. 2003).

Although we have broadly ranked control strategies by their potential to harm ecosystems and host populations, we have not incorporated the intensity of any proposed control. Low-intensity control programs may reduce the detrimental effects of control, because they reduce the parasite burden without causing local parasite extinction. For instance, vaccination programs that target geographic corridors of transmission (Haydon et al. 2006) or that target vulnerable or important individuals not only limit the effects of control but also leave a portion of a population to serve as sentinels for future disease detection.

No single parasite control method will be effective for all parasites. For instance, many parasites do not have reservoir hosts in either wildlife or livestock, or they use intermediate hosts. Reducing host density may only reduce parasite abundance where abundance is determined by density-dependent rather than frequency-dependent transmission.

\section{Past examples of parasite control}

The literature shows that many past examples of parasite control have been in critically endangered species, with parasites that cause mortality. The most common form of parasite control in these situations is vaccination (Woodroffe 1999, Wobeser 2002). These examples broadly concur with our proposed first principles. However, there are a number of examples in the literature that do not.

The African buffalo (Syncerus caffer) is listed on the IUCN Red List as a species of least concern. In South Africa, a recent government-funded program created a number of "disease-free" buffalo populations. The populations have been bred to be free from two introduced parasites (bovine tuberculosis and brucellosis) and two native parasites (footand-mouth disease and corridor disease). The justification for this program was specifically to "save the large gene pool of the Kruger Park buffalo" (Laubscher and Hoffman 2012). Corridor disease is a subclinical infection in buffalo caused by the piroplasm Theileria parva. Using the first principles proposed here, there is no need to control a subclinical parasite in a host species of least concern beyond ecological restoration. We also argue that the large gene pool of the Kruger Park buffalo population provides excellent conditions for the coevolution of buffalo with their parasites. The eradication of this parasite from new populations of buffalo may have a range of ecological consequences. For instance, the naivety of these new populations to T. parva may mean that they become susceptible to the fatal infections caused by T. parva in cattle. 


\begin{tabular}{|c|c|c|c|}
\hline Type of parasite control & Mechanism & Notes & References \\
\hline Pollution reduction ${ }^{a}$ & $\begin{array}{l}\text { Reduce exposure to pollutants that } \\
\text { may affect host immune system } \\
\text { functioning }\end{array}$ & Ecological restoration & Smith et al. 2009 \\
\hline $\begin{array}{l}\text { Buffer zone between wildlife and } \\
\text { livestock }\end{array}$ & $\begin{array}{l}\text { Reduce transmission between } \\
\text { domestic livestock and wildlife }\end{array}$ & Ecological restoration & Pedersen et al. 2007 \\
\hline Livestock vaccination ${ }^{a}$ & $\begin{array}{l}\text { Reduce susceptible host density (of } \\
\text { reservoir) and, therefore, parasite } \\
\text { abundance }\end{array}$ & Ecological restoration & Pedersen et al. 2007 \\
\hline Reintroduce parasitic competitors ${ }^{a}$ & $\begin{array}{l}\text { Increased competition with the } \\
\text { pathogenic parasite species to } \\
\text { reduce its abundance }\end{array}$ & Ecological restoration & Lello et al. 2004 \\
\hline $\begin{array}{l}\text { Host translocation in, improve } \\
\text { connectivity between populations }\end{array}$ & $\begin{array}{l}\text { Reduce inbreeding depression and, } \\
\text { therefore, host susceptibility to } \\
\text { parasitism }\end{array}$ & Ecological restoration & Smith et al. 2009 \\
\hline Remove dead hosts ${ }^{b}$ & $\begin{array}{l}\text { Carcases are used for reinfection by } \\
\text { some parasites }\end{array}$ & $\begin{array}{l}\text { Ecologically invasive, removes key link } \\
\text { in food web }\end{array}$ & Gates et al. 1995 \\
\hline Host translocation out ${ }^{\mathrm{b}}$ & $\begin{array}{l}\text { Reduce susceptible host density and, } \\
\text { therefore, parasite abundance }\end{array}$ & $\begin{array}{l}\text { Ecologically invasive, reduce } \\
\text { ecosystem roles of host }\end{array}$ & Wobeser 2002 \\
\hline Cull or vaccinate sympatric hosts ${ }^{b}$ & $\begin{array}{l}\text { Reduce susceptible host density (of } \\
\text { reservoir) and, therefore, parasite } \\
\text { abundance }\end{array}$ & $\begin{array}{l}\text { Ecologically invasive, reduces } \\
\text { ecosystem roles or alters host- } \\
\text { parasite evolution in sympatric host }\end{array}$ & Gurnell et al. 2006 \\
\hline $\begin{array}{l}\text { Reduce vector population through } \\
\text { habitat modification or chemical } \\
\text { trapping" }\end{array}$ & $\begin{array}{l}\text { Reduces transmission rate of } \\
\text { parasite and, therefore, parasite } \\
\text { abundance }\end{array}$ & $\begin{array}{l}\text { Ecologically invasive, reduces vector } \\
\text { ecosystem roles and increases } \\
\text { likelihood of local vector extinction }\end{array}$ & Fyumagwa et al. 2007 \\
\hline Vaccination $^{c}$ & $\begin{array}{l}\text { Reduce susceptible host density, } \\
\text { directly protect threatened species }\end{array}$ & Alter host-parasite evolution & Woodroffe 1999 \\
\hline Treatment $^{c}$ & Eliminate parasite from treated hosts & $\begin{array}{l}\text { Alter host-parasite evolution, impacts } \\
\text { on nontarget parasite species }\end{array}$ & Spratt 1997 \\
\hline
\end{tabular}

Bighorn sheep (Ovis canadensis) are also listed on the IUCN Red List as a species of least concern. In the $1970 \mathrm{~s}$, the broad-spectrum anthelmintic Fenbendazole was used to treat populations of bighorn sheep for the lungworm Protostrongylus spp. It was proposed that the native lungworm was making $O$. canadensis more susceptible to bacterial pneumonia. The fundamental thinking behind this control program clearly differs from our proposed first principles. The use of a broad-spectrum anthelmintic in a host species of least concern to purportedly control a parasite through an unfounded mechanism (Miller et al. 2000) shows scant regard for the value of parasites within an ecosystem. Reducing the transmission rate of bacterial pneumonia between livestock and wildlife for ecological restoration might have been wiser.

The African elephant (Loxodonta africana) is listed on the IUCN Red List as vulnerable to extinction. An estimated 7500 elephants live in the Kruger National Park in South Africa. Between 1987 and 1994, there was an outbreak of encephalomyocarditis virus that caused the deaths of 64 individuals (Grobler et al. 1995). This native parasite is thought to be predominantly carried by myomorph rodents. A vaccine was developed with the specific aim of protecting elephants from outbreaks of the disease (Hunter et al.

http://bioscience.oxjordjournals.org
1998). Using the first principles proposed here, there may be a need to control those parasites that have a very high impact on host mortality. However, this single outbreak over a long period may reflect the normal evolution of the host and does not threaten long-term population stability Vaccination may exacerbate the susceptibility of elephants to the disease, because it will create a population less resistant to encephalomyocarditis virus without controlling the disease itself (which is maintained primarily within rodents). In the event of a larger outbreak that is of concern, the control or vaccination of the rodent population may be more prudent.

These three examples demonstrate an overestimation of the threat of parasites and an underestimation of the importance of parasites within an ecological community and of the value of parasite biodiversity. In contrast, there are some management plans that actively address parasite conservation. For instance, the black rhino is recolonizing much of its former range in east Africa. However, there were concerns that the host-specific black rhino bot fly, Gyrostigma rhinocerontis, was not recolonizing certain populations. The Kenyan black rhino management plan of 1993 proposed translocating black rhino infected with the parasite into areas where it had not recolonized (Brett 1993). This is an 
example of active parasite conservation-reintroducing parasites from parasite refugia into newly established host populations.

\section{Conclusions}

In this article, we aim to provide a rational and robust method of deciding when and how to control parasitism in threatened species, which, we believe, will help conservation managers avoid unwanted outcomes. The proposed first principles will allow reserve managers to tailor parasite control strategies to their own populations. 'This is importan because a parasite's impact on a population's performance may change depending on the strain of the parasite (Lively and Dybdahl 2000), and the host may be more susceptible in certain populations because of nutritional or physiological stress (Cornet et al. 2014). We actively encourage discussion of the parasites of threatened species, because we believe that there are numerous avenues for further debate. For instance, these first principles do not incorporate the phylogenetic distinctness of the host or the parasite species (Faith et al. 2004). They also rely on the IUCN Red List, which is currently highly biased toward vertebrates (Whiteman and Parker 2005). Further experimental research is needed on the potentially detrimental effects of controlling parasitism-for example, by leaving a subset of host populations as control populations, free from intervention.

\section{Acknowledgements}

The authors are grateful to Stephen Hartley, Nancy Webber, colleagues at Victoria University of Wellington, and anonymous reviewers for their insightful comments and recommendations.

\section{References cited}

Acevedo-Whitehouse K, Gulland F, Greig D, Amos W. 2003. Inbreeding Disease susceptibility in California sea lions. Nature 422: 35 .

Almberg ES, Cross PC, Dobson AP, Smith DW, Hudson PJ. 2012. Parasite invasion following host reintroduction: A case study of Yellowstone wolves. Philosophical Transactions of the Royal Society B 367: 2840-2851.

Altizer S, Nunn CL, Lindenfors P. 2007. Do threatened hosts have fewer parasites? A comparative study in primates. Journal of Animal Ecology 76: $304-314$

Altizer S, Ostfeld RS, Johnson P'Tl, Kutz S, Harvell CD. 2013. Climate change and infectious diseases: From evidence to a predictive framework. Science 341: 514-519.

Brett RA. 1993. Conservation Strategy and Management Plan for the Black Rhinoceros (Diceros bicornis) in Kenya. Kenya Wildlife Service.
Rha. 1993. Conservation Strategy and Management Plan for the

Burrows R, Hofer H, East ML. 1995. Population dynamics, intervention and survival in African wild dogs (Lycaon pictus). Proceedings of the Royal Society B 262:235-245.

Cassinello J, Gomendio M, Roldan FRS. 2001. Relationship between coefficient of inbreeding and parasite burden in endangered gazelle Conservation Biology 15: 1171-1174.

Chauvenet ALM, Durant SM, Hilborn R, Pettorelli N. 2011. Unintended consequences of conservation actions: Managing disease in complex ecosystems. PLOS ONE 6 (art. e28671).

Colwell RK, Dunn RR, Harris NC. 2012. Coextinction and persistence of dependent species in a changing world. Annual Review of Ecology Evolution, and Systematics 43: 183-203.
Cornet S, Bichet C, Larcombe S, Faivre B, Sorci G. 2014. Impact of host nutritional status on infection dynamics and parasite virulence in a bird-malaria system. Journal of Animal Ecology 83: 256-265.

Dunn RR, Harris NC, Colwell RK, Koh LP, Sodhi NS. 2009. The sixth mass coextinction: Are most endangered species parasites and mutualists? Proceedings of the Royal Society B 276: 3037-3045.

Faith DP, Reid CAM, Hunter J. 2004. Integrating phylogenetic diversity, complementarity, and endemism for conservation assessment. Sily, complementarity, and endemi
Conservation Biology 18: 255-261.

Frick WF, Pollock JF, Hicks AC, Langwig KE, Reynolds DS, 'Turner GG, Frick WF, Pollock JF, Hicks AC, Langwig KE, Reynolds DS, lurner GG,
Butchkoski CM, Kunz TH. 2010. An emerging disease causes regional population collapse of a common North American bat species. Science 329: 679-682.

Fyumagwa RD, Runyoro V, Horak IG, Hoare R. 2007. Ecology and control of ticks as disease vectors in wildlife of the Ngorongoro Crater, Tanzania. South African Journal of Wildlife Research 37: 79-90.

Gandon S, Mackinnon M, Nee S, Read A. 2003. Imperfect vaccination: Some epidemiological and evolutionary consequences. Proceedings of the Royal Society B 270: 1129-1136.

Gates CC, Elkin BT, Dragon DC. 1995. Investigation, control, and epizootiology of anthrax in a geographically isolated, free-roaming bison population in northern Canada. Canadian Journal of Veterinary Research 59: 256-264.

Gómez A, Nichols F. 2013. Neglected wild life: Parasitic biodiversity as a conservation target. International Journal for Parasitology: Parasites and Wildlife 2: 222-227.

Grobler DG, Raath IP, Braack LL, Keet DF, Gerdes GH, Barnard BJ, Kriek NP, Jardine J, Swanepoel R. 1995. An outbreak of encephalomyocarditisvirus infection in free-ranging African elephants in the Kruger National Park. Onderstepoort Journal of Veterinary Research 62: 97-108

Gurnell J, Rushton SP, Lurz PWW, Sainsbury AW, Nettleton P, Shirley MDI Bruemmer C, Geddes N. 2006. Squirrel poxvirus: Landscape scale strategies for managing disease threat. Biological Conservation 131: 287-295.

Harris NC, Livieri TM, Dunn RR. 2014. Ectoparasites in black-footed ferrets (Mustela nigripes) from the largest reintroduced population of the Conata Basin, South Dakota, USA. Journal of Wildlife Diseases 50: 340-343.

Haydon DT, et al. 2006. I.ow-coverage vaccination strategies for the conservaydon DT, et al. 2006. Low-coverage vaccination strate
vation of endangered species. Nature 443: 692-695.

vation of Sndangered species. Nature 443: 692-695.
Heard MJ, Smith KF, Ripp KJ, Berger M, Chen J, Dittmeier J, Goter M, McGarvey SI, Kyan E. 2013. 'The threat of disease increases as species
Mct, McGarvey SI, Ryan E. 2013. The threat of disease increases as
move toward extinction. Conservation Biology 27: 1378-1388.

Hudson PJ, Dobson AP, Lafferty KD. 2006. Is a healthy ecosystem one that is rich in parasites? Trends in Ecology and Evolution 21: 381-385.

Hunter P, Swanepoel SP, Esterhuysen JJ, Raath JP, Bengis RG, Van der Lugt JJ. 1998. The efficacy of an experimental oil-adjuvanted encephalomyocarditis vaccine in elephants, mice, and pigs. Vaccine 16: 55-61.

Irvine RI. 2006. Parasites and the dynamics of wild mammal populations. Animal Science 82: 775-781.

Johnson S, Gerding DN. 1998. Clostridium difficile-associated diarrhea. Clinical Infectious Diseases 26: 1027-1036

Knobel DL, Fooks AR, Brookes SM, Randall DA, Williams SD, Argaw K, Shiferaw F, Tallents LA, Laurenson MK. 2008. Trapping and vaccination of endangered Ethiopian wolves to control an outbreak of rabies. Journal of Applied Fcology 45: 109-116.

Koh LP, Dunn RD, Sodhi NS, Colwell RK, Yroctor HC, Smith VS. 2004. Species co-extinctions and the biodiversity crisis. Science 305: 1632-1634.

Lafferty KD, Gerber LR. 2002. Good medicine for conservation biology: The intersection of epidemiology and conservation theory. Conservation Biology 16: 593-604.

Laubscher L, Hoffman L. 2012. An overview of disease-free buffalo breeding projects with reference to the different systems used in South Africa. Sustainability 4: 3124-3140.

Lello J, Boag B, Fenton A, Stevenson IR, Hudson PJ. 2004. Competition and mutualism among the gut helminths of a mammalian host. Nature 428 : mutualis. 
Appendix 4

Forum

Lively CM, Dybdahl MF. 2000. Parasite adaptation to locally common host genotypes. Nature 405: 679-681.

Martinez-Padilla J, Redpath SM, Zeineddine M, Mougeot F. 2014. Insights into population ecology from long-term studies of red grouse Lagopus lagopus scoticus. Journal of Animal Ecology 83: 85-98.

Mihok S, Kock R, Masake R. 1995. Health implications of translocation of endangered species in Africa: Trypanosomiasis in rhinoceros. Page 423-424 in Proceedings of the Joint Conference of American Association 423-424 Troceedings of the es Disence Association of Willese Neterinarians, Lansing, Michign Asmerican Association of Zoo Veterinarians, Wildife Discase Assoc American Association of Wildlife Veterinarian

Miller MW, Vayhinger JE, Bowden DC, Roush SP, Verry TE, Torres AN, Jurgens VD. 2000. Drug treatment for lungworm in bighorn sheep Reevaluation of a 20-year-old management prescription. Journal of Wildlife Management 64: 505-512.

Moir ML, Vesk PA, Brennan KEC, Poulin R, Hughes L, Keith DA, McCarthy MA, Coates DI. 2012. Considering extinction of dependent specics during translocation, ex situ conservation, and assisted migration of threatened hosts. Conservation Biology 26: 199-207.

Nunn CL, Altizer S, Sechrest W, Jones KE, Barton RA, Gittleman JL. 2004. Parasites and the evolutionary diversification of primate clades. American Naturalist 164 (suppl.): S90-S103.

Pedersen AB, Jones KF, Nunn CT, Altizer S. 2007. Infectious diseases and extinction risk in wild mammals. Conservation Biology 21: 1269-1279.
Randall DA, et al. 2004. Rabies in endangered Ethiopian wolves. Emerging Infectious Diseases 10: 2214-2217.

Rudgers JA, Miller TEX, Ziegler SM, Craven KD. 2012. There are many ways to be a mutualist: Endophytic fungus reduces plant survival but increases population growth. Ecology 93: 565-574.

Schwanz LE. 2008. Persistent effects of maternal parasitic infection on offspring fitness: Implications for adaptive reproductive strategies when parasitized. Functional Ecology 22: 691-698.

Smith $\mathrm{KF}$ Aceredo-Whitehour $\mathrm{K}$, Pederen $\mathrm{AB}$. 2009 . The rote of infecTion, Acevedo-Whitehouse K, Pedersen AB. 2009 . The jole of infections Arese Spratt DM. 1997. Endoparasite control strategies: Implications for biodiversity of native fauna. International Journal of Parasitology 27: 173-180. Whiteman NK, Parker PG. 2005. Using parasites to infer host population history: A new rationale for parasite conservation. Animal Conservation 8: 175-181.

Wobeser G. 2002. Disease management strategies for wildlife. OIE Revue Scientifique et Technique 21: 159-178,

Woodroffe R. 1999. Managing disease threats to wild mammals. Animal Conservation 2: 185-193

Andrew Paul Stringer and Wayne Iinklater are affiliated with the School of Biological Sciences at Victoria University of Wellington, in Wellington, New Biological Sciences at Victoria University of Wellinglon, in Wellinglon, New
7.ealand. WL is also affililated with the Centre for African Conservation Fcology 7ealand. WT is also affililated with the Centre for African Conservation Fcolog
at Nelson Mandela Metropolitan University in Port Llizabeth, South Africa. 